\title{
Organocatalysis Linked to Charge-Enhanced Acidity with Superelectrophilic Traits
}

Ivor Smajlagic, Brandon White, Oyindamola Azeez, Melanie Pilkington and Travis Dudding*

Department of Chemistry, Brock University; St. Catharines, L2S 3A1, Canada.

Email: tdudding@,brocku.ca

\section{Supplementary Materials}

\section{Contents:}

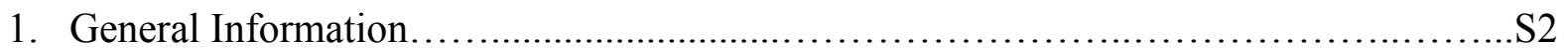

2. Variable Time Normalization Analysis......................................... 3

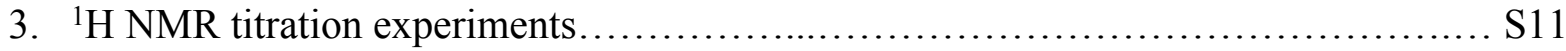

4. Synthetic procedures and characterization data of catalysts and products.............S12

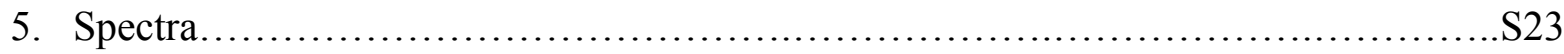

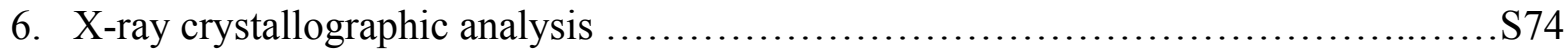

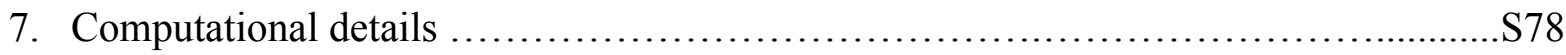

8. Cartesian coordinates and energies of calculated structures....................... 79

9. Parameters for OPLS4 conformer searches.................................... 128

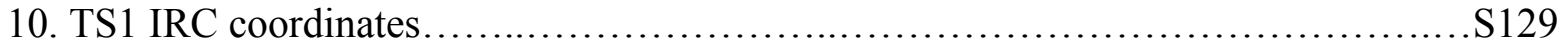

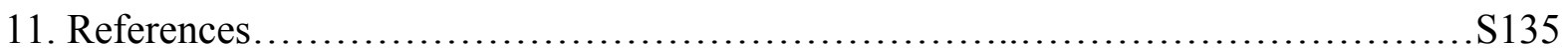




\section{General information}

All materials were obtained from commercially available suppliers and used as received unless otherwise stated. Dichloromethane (DCM) was distilled from $\mathrm{CaH}_{2}$. All reactions were conducted in oven-dried round-bottom flasks under an inert atmosphere $\left(\mathrm{N}_{2}\right)$ and monitored by thin layer chromatography (TLC) using silica gel 60 F254 EMD Merck, whilst visualized with a hand-held UV lamp. Flash column chromatography was performed over Silicycle ultrapure silica gel (230400 mesh). NMR spectra were recorded on a Bruker DPX-300 spectrometer $\left({ }^{1} \mathrm{H} 300 \mathrm{MHz}\right.$, ${ }^{13} \mathrm{C}\left\{{ }^{1} \mathrm{H}\right\}$ 75.5 MHz, $\left.{ }^{19} \mathrm{~F}\left\{{ }^{1} \mathrm{H}\right\} 292.4 \mathrm{MHz},{ }^{11} \mathrm{~B}\left\{{ }^{1} \mathrm{H}\right\} 96.3 \mathrm{MHz}\right)$ and Bruker Avance AV I 600 spectrometer $\left({ }^{1} \mathrm{H} 600 \mathrm{MHz}\right)$ in $\mathrm{CDCl}_{3}$. The observed chemical shifts are reported as $\delta$-values (ppm) relative to tetramethylsilane (TMS). 1D NOESY NMR experiments were used to assist in $Z / E$ isomer identification. Mass spectra were obtained on a MSI/Kratos concept IS Mass spectrometer. Oximes $^{1-14}$, 2,3 bis(diisopropylamino)cyclopropenimine, ${ }^{15}$ 3,4-bis(cyclopentyloxy)cyclobut-3ene-1,2-dione, ${ }^{16}$ pyridinium tetrafluoroborate, ${ }^{17}$ 1-phenylethyl-2,2,2-trichloroacetimidate, ${ }^{18}$ and 2,3,4,6-tetra- $O$ - $\alpha$-D-galactopyranosyl trichloroacetimidate ${ }^{19}$ were prepared according to literature procedures and the spectra match accordingly. 


\section{Variable Time Normalization Analysis (VTNA)}

\section{Procedure for determining potential product inhibition or catalyst deactivation.}

Two independent reactions were conducted in oven-dried NMR tubes. The first reaction was charged with 3,4-dihydro-2H-pyran (6a) $(1.35 \mathrm{M})$ to which was diluted in $0.5 \mathrm{~mL} \mathrm{CDCl}_{3}$. Next, the addition of acetophenone oxime $(5 \mathbf{a})(0.68 \mathrm{M})$ and catalyst $\mathbf{4 e} \cdot \mathbf{B F}_{4}^{-}(5 \mathrm{~mol} \% ; 0.034 \mathrm{M})$ were added to the solution. The next experiment followed the same protocol; however, with a consistent reduction in the initial concentrations of substrates by $0.16 \mathrm{M}$, i.e., $\mathbf{6 a}=1.19 \mathrm{M}$ and $\mathbf{5 a}=0.52 \mathrm{M}$. Each respective experiment was placed in a ${ }^{1} \mathrm{H}$ NMR $300 \mathrm{MHz}$ spectrometer and allowed to react for $\sim 5$ hours at $40{ }^{\circ} \mathrm{C}$. Reaction progress was tracked by monitoring the disappearance of the signal at $\sim 7.65 \mathrm{ppm}$ for $\mathbf{5 a}$ and appearance of the signal at $\sim 7.71 \mathrm{ppm}$ for product $7 \mathbf{a}$. Data points were recorded every six minutes.

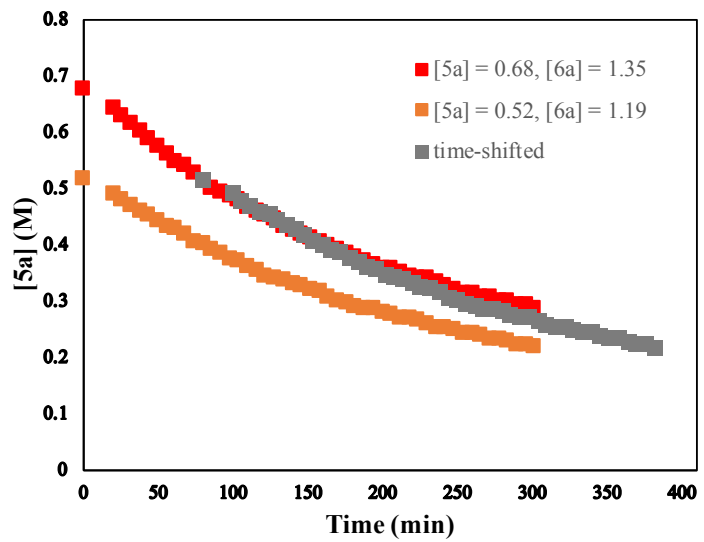

Figure S1. Plot depicting the absence of catalyst deactivation and product inhibition.

Table S1. Data for the determination of the potential product inhibition or catalyst deactivation.

\begin{tabular}{|c|c|c|c|c|c|}
\hline \multicolumn{2}{|c|}{ expt 1: [5a] = 0.68 M, [6a] } & \multicolumn{3}{|c|}{ expt 2: [5a] = 0.52 M, [6a] = 1.19 M } \\
\hline 1.35 M & [5a] (M) & time (min) & [5a] (M) & time-shift & [5a] (M) \\
\hline time (min) & 0.68 & 0 & 0.52 & 80 & 0.52 \\
20 & 0.646 & 20 & 0.494 & 100 & 0.494 \\
26 & 0.6324 & 26 & 0.4836 & 106 & 0.4836 \\
32 & 0.6188 & 32 & 0.4732 & 112 & 0.4732 \\
38 & 0.6052 & 38 & 0.4628 & 118 & 0.4628 \\
44 & 0.5916 & 44 & 0.4576 & 124 & 0.4576 \\
50 & 0.578 & 50 & 0.4472 & 130 & 0.4472 \\
56 & 0.5644 & 56 & 0.4368 & 136 & 0.4368 \\
62 & 0.5508 & 62 & 0.4316 & 142 & 0.4316
\end{tabular}




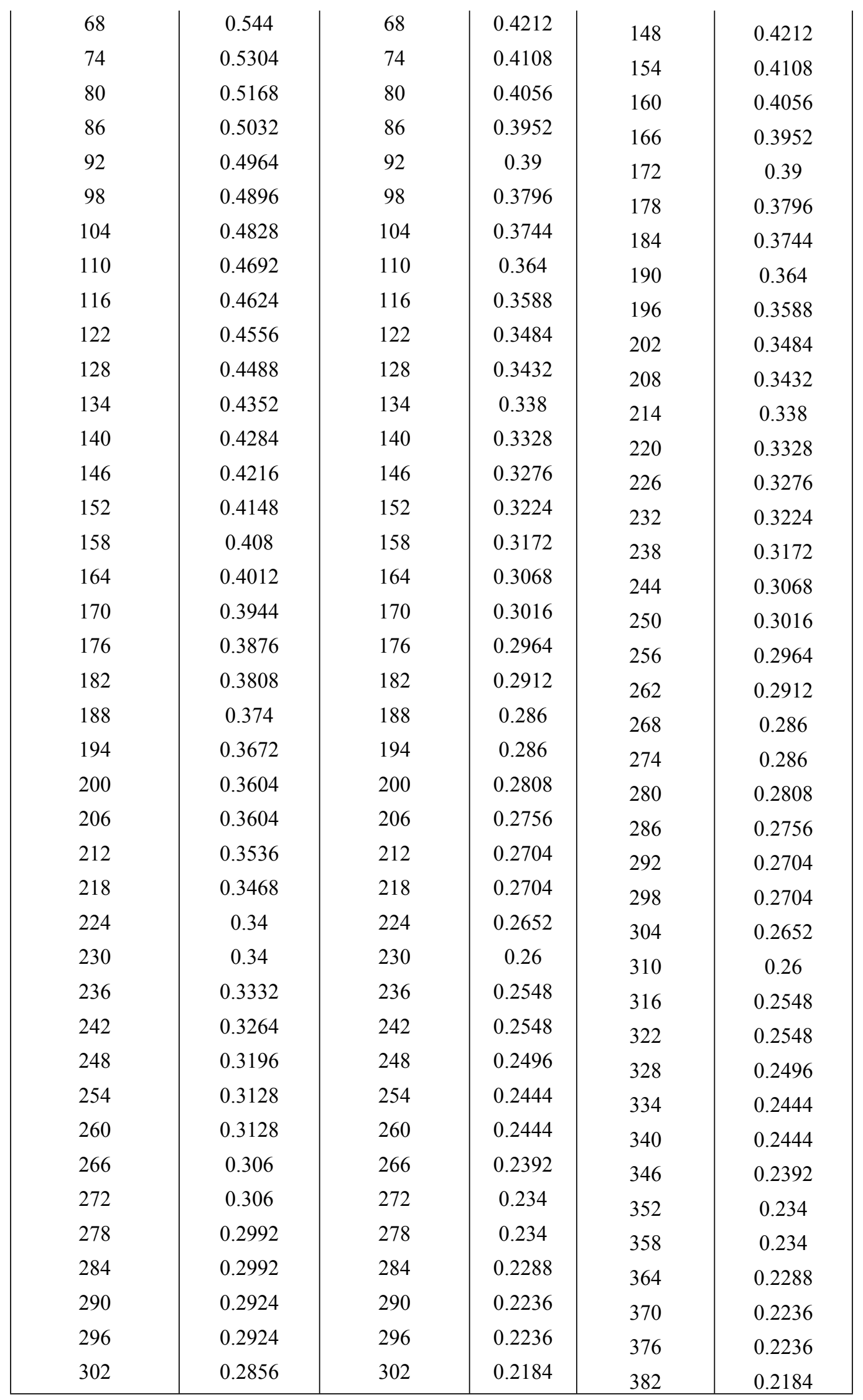




\section{Representative procedure for determining the order in catalyst.}

Using the data obtained from the first experiment (see above), two additional independent reactions were conducted in oven-dried NMR tubes charged with 3,4-dihydro-2H-pyran (6a) (1.35 M) to which were diluted in $0.5 \mathrm{~mL} \mathrm{CDCl}{ }_{3}$. Next, acetophenone oxime $(\mathbf{5 a})(0.68 \mathrm{M})$ and catalyst $4 \mathbf{e}^{-} \mathbf{B F}_{4}{ }^{-}$(2.5- or $7.5 \mathrm{~mol} \%$; 0.015 - or $0.053 \mathrm{M}$, respectively) were added to the solutions. Each respective experiment was placed in a ${ }^{1} \mathrm{H}$ NMR $300 \mathrm{MHz}$ spectrometer and allowed to react for $3-4$ hours at $40{ }^{\circ} \mathrm{C}$. Reaction progress was tracked by monitoring the disappearance of the signal at $\sim 7.65 \mathrm{ppm}$ for $\mathbf{5 a}$ and appearance of the signal at $\sim 7.71 \mathrm{ppm}$ for product $7 \mathbf{a}$. Data points were recorded every six minutes.

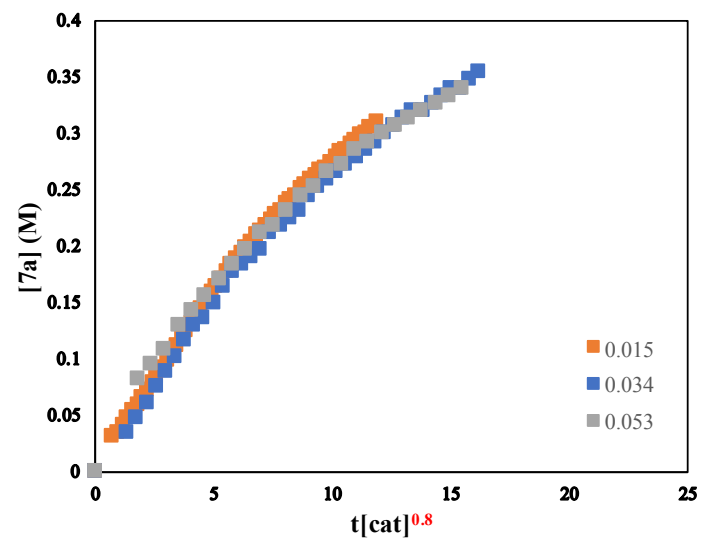

Figure S2. Normalized time scale plot displaying a 0.8 -order dependence in catalyst.

Table S2. Data for the determination of the reaction order in catalyst.

\begin{tabular}{|c|c|c|c|c|c|c|c|c|c|c|c|}
\hline \multicolumn{4}{|c|}{ catalyst loading: $2.5 \mathrm{~mol} \%$} & \multicolumn{4}{|c|}{ catalyst loading: 5 mol\% } & \multicolumn{4}{|c|}{ catalyst loading: 7.5 mol\% } \\
\hline $\begin{array}{l}\text { time } \\
\text { (min) }\end{array}$ & $\begin{array}{l}{[7 \mathbf{a}]} \\
(\mathrm{M})\end{array}$ & $\begin{array}{c}{\left[\mathbf{4 e} \cdot \mathbf{B F}_{4}^{-}\right]} \\
(\mathrm{M})\end{array}$ & $\begin{array}{c}\text { tcat }^{\gamma} \\
\gamma=0.8\end{array}$ & $\begin{array}{l}\text { time } \\
(\min )\end{array}$ & $\begin{array}{l}{[7 \mathbf{a}]} \\
(\mathrm{M})\end{array}$ & $\begin{array}{c}{\left[\mathbf{4 e} \cdot \mathbf{B F}_{4^{-}}\right]} \\
(\mathrm{M})\end{array}$ & $\begin{array}{c}\text { tcat }^{\gamma} \\
\gamma=0.8\end{array}$ & $\begin{array}{l}\text { time } \\
(\min )\end{array}$ & $\begin{array}{l}{[7 \mathrm{a}]} \\
\text { (M) }\end{array}$ & $\begin{array}{c}{\left[4 \mathbf{e} \cdot \mathbf{B F}_{4}^{-}\right]} \\
(\mathrm{M})\end{array}$ & $\begin{array}{c}\text { tcat }^{\gamma} \\
\gamma=0.8\end{array}$ \\
\hline 0 & 0 & 0.015 & 0 & 0 & 0 & 0.034 & 0 & 0 & 0 & 0.053 & 0 \\
\hline 36 & 0.030464 & 0.015 & 0.72 & 20 & 0.034 & 0.034 & 1.34 & 32 & 0.0816 & 0.053 & 1.753 \\
\hline 42 & 0.03502 & 0.015 & 0.93 & 26 & 0.0476 & 0.034 & 1.74 & 38 & 0.0952 & 0.053 & 2.325 \\
\hline 48 & 0.041412 & 0.015 & 1.14 & 32 & 0.0612 & 0.034 & 2.14 & 44 & 0.1088 & 0.053 & 2.897 \\
\hline 54 & 0.0476 & 0.015 & 1.34 & 38 & 0.0748 & 0.034 & 2.54 & 50 & 0.1292 & 0.053 & 3.47 \\
\hline 60 & 0.053516 & 0.015 & 1.55 & 44 & 0.0884 & 0.034 & 2.94 & 56 & 0.1428 & 0.053 & 4.042 \\
\hline 66 & 0.05916 & 0.015 & 1.76 & 50 & 0.102 & 0.034 & 3.34 & 62 & 0.1564 & 0.053 & 4.614 \\
\hline 72 & 0.065144 & 0.015 & 1.97 & 56 & 0.1156 & 0.034 & 3.74 & 68 & 0.17 & 0.053 & 5.186 \\
\hline 78 & 0.0714 & 0.015 & 2.18 & 62 & 0.1292 & 0.034 & 4.15 & 74 & 0.1836 & 0.053 & 5.758 \\
\hline 84 & 0.0782 & 0.015 & 2.39 & 68 & 0.136 & 0.034 & 4.55 & 80 & 0.1972 & 0.053 & 6.331 \\
\hline 90 & 0.084116 & 0.015 & 2.59 & 74 & 0.1496 & 0.034 & 4.95 & 86 & 0.2108 & 0.053 & 6.903 \\
\hline 96 & 0.0918 & 0.015 & 2.8 & 80 & 0.1632 & 0.034 & 5.35 & 92 & 0.2176 & 0.053 & 7.475 \\
\hline 102 & 0.09826 & 0.015 & 3.01 & 86 & 0.1768 & 0.034 & 5.75 & 98 & 0.2312 & 0.053 & \\
\hline
\end{tabular}




\begin{tabular}{|c|c|c|c|c|c|c|c|c|c|c|c|}
\hline 108 & 0.10472 & 0.015 & 3.22 & 92 & 0.1836 & 0.034 & 6.15 & 104 & 0.2448 & 0.053 & 8.62 \\
\hline 114 & 0.11084 & 0.015 & 3.43 & 98 & 0.1904 & 0.034 & 6.55 & 110 & 0.2516 & 0.053 & 9.192 \\
\hline 120 & 0.117844 & 0.015 & 3.64 & 104 & 0.1972 & 0.034 & 6.95 & 116 & 0.2652 & 0.053 & 9.764 \\
\hline 126 & 0.12444 & 0.015 & 3.85 & 110 & 0.2108 & 0.034 & 7.35 & 122 & 0.272 & 0.053 & 10.34 \\
\hline 132 & 0.13124 & 0.015 & 4.05 & 116 & 0.2176 & 0.034 & 7.76 & 128 & 0.2856 & 0.053 & 10.91 \\
\hline 138 & 0.13804 & 0.015 & 4.26 & 122 & 0.2244 & 0.034 & 8.16 & 134 & 0.2924 & 0.053 & 11.48 \\
\hline 144 & 0.14484 & 0.015 & 4.47 & 128 & 0.2312 & 0.034 & 8.56 & 140 & 0.2992 & 0.053 & 12.05 \\
\hline 150 & 0.15096 & 0.015 & 4.68 & 134 & 0.2448 & 0.034 & 8.96 & 146 & 0.306 & 0.053 & 12.63 \\
\hline 156 & 0.15844 & 0.015 & 4.89 & 140 & 0.2516 & 0.034 & 9.36 & 152 & 0.3128 & 0.053 & 13.2 \\
\hline 162 & 0.16422 & 0.015 & 5.1 & 146 & 0.2584 & 0.034 & 9.76 & 158 & 0.3196 & 0.053 & 13.77 \\
\hline 168 & 0.17 & 0.015 & 5.3 & 152 & 0.2652 & 0.034 & 10.2 & 164 & 0.3264 & 0.053 & 14.34 \\
\hline 174 & 0.1768 & 0.015 & 5.51 & 158 & 0.272 & 0.034 & 10.6 & 170 & 0.3332 & 0.053 & 14.91 \\
\hline 180 & 0.1836 & 0.015 & 5.72 & 164 & 0.2788 & 0.034 & 11 & 176 & 0.34 & 0.053 & 15.49 \\
\hline 186 & 0.18836 & 0.015 & 5.93 & 170 & 0.2856 & 0.034 & 11.4 & - & - & - & - \\
\hline 192 & 0.19312 & 0.015 & 6.14 & 176 & 0.2924 & 0.034 & 11.8 & - & - & - & - \\
\hline 198 & 0.19856 & 0.015 & 6.35 & 182 & 0.2992 & 0.034 & 12.2 & - & - & - & - \\
\hline 204 & 0.20332 & 0.015 & 6.56 & 188 & 0.306 & 0.034 & 12.6 & - & - & - & - \\
\hline 210 & 0.20944 & 0.015 & 6.76 & 194 & 0.3128 & 0.034 & 13 & - & - & - & - \\
\hline 216 & 0.21352 & 0.015 & 6.97 & 200 & 0.3196 & 0.034 & 13.4 & - & - & - & - \\
\hline 222 & 0.21828 & 0.015 & 7.18 & 206 & 0.3196 & 0.034 & 13.8 & - & - & - & - \\
\hline 228 & 0.22304 & 0.015 & 7.39 & 212 & 0.3264 & 0.034 & 14.2 & - & - & - & - \\
\hline 234 & 0.22814 & 0.015 & 7.6 & 218 & 0.3332 & 0.034 & 14.6 & - & - & - & - \\
\hline 240 & 0.23188 & 0.015 & 7.81 & 224 & 0.34 & 0.034 & 15 & - & - & - & - \\
\hline 246 & 0.23732 & 0.015 & 8.01 & 230 & 0.34 & 0.034 & 15.4 & - & - & - & - \\
\hline 252 & 0.2414 & 0.015 & 8.22 & 236 & 0.3468 & 0.034 & 15.8 & - & - & - & - \\
\hline 258 & 0.24412 & 0.015 & 8.43 & 242 & 0.3536 & 0.034 & 16.2 & - & - & - & - \\
\hline 264 & 0.25092 & 0.015 & 8.64 & - & - & - & - & - & - & - & - \\
\hline 270 & 0.25364 & 0.015 & 8.85 & - & - & - & - & - & - & - & - \\
\hline 276 & 0.2584 & 0.015 & 9.06 & - & - & - & - & - & - & - & - \\
\hline 282 & 0.26248 & 0.015 & 9.27 & - & - & - & - & - & - & - & - \\
\hline 288 & 0.26724 & 0.015 & 9.47 & - & - & - & - & - & - & - & - \\
\hline 294 & 0.26928 & 0.015 & 9.68 & - & - & - & - & - & - & - & - \\
\hline 300 & 0.27404 & 0.015 & 9.89 & - & - & - & - & - & - & - & - \\
\hline 306 & 0.278664 & 0.015 & 10.1 & - & - & - & - & - & - & - & - \\
\hline 312 & 0.28288 & 0.015 & 10.3 & - & - & - & - & - & - & - & - \\
\hline 318 & 0.2856 & 0.015 & 10.5 & - & - & - & - & - & - & - & - \\
\hline 324 & 0.29036 & 0.015 & 10.7 & - & - & - & - & - & - & - & - \\
\hline 330 & 0.29308 & 0.015 & 10.9 & - & - & - & - & - & - & - & - \\
\hline 336 & 0.29784 & 0.015 & 11.1 & - & - & - & - & - & - & - & - \\
\hline 342 & 0.30056 & 0.015 & 11.3 & - & - & - & - & - & - & - & - \\
\hline
\end{tabular}




\begin{tabular}{|l|l|l|l|l|l|l|l|l|l|l|l|l|l|}
348 & 0.30464 & 0.015 & 11.6 & - & - & - & - & - & - & - & - \\
356 & 0.31008 & 0.015 & 11.8 & - & - & - & - & - & - & - & - \\
\hline
\end{tabular}

\section{Procedure for determining the oxime reaction order dependence}

Using the data obtained from the first experiment (see above), two additional independent reactions were conducted in oven-dried NMR tubes charged with 3,4-dihydro- $2 H$-pyran (6a) (1.35 $\mathrm{M})$ to which were diluted in $0.5 \mathrm{~mL} \mathrm{CDCl}_{3}$. Next, acetophenone oxime (5a) (0.44- or $\left.0.56 \mathrm{M}\right)$ and catalyst $\mathbf{4 e} \cdot \mathbf{B F}_{4}^{-}(5 \mathrm{~mol} \% ; 0.034 \mathrm{M})$ were added to the solutions. Each respective experiment was placed in a ${ }^{1} \mathrm{H}$ NMR $300 \mathrm{MHz}$ spectrometer and allowed to react for $\sim 4$ hours at $40^{\circ} \mathrm{C}$. Reaction progress was tracked by monitoring the disappearance of the signal at $\sim 7.65 \mathrm{ppm}$ for 5a and appearance of the signal at $\sim 7.71 \mathrm{ppm}$ for product $7 \mathbf{a}$. Data points were recorded every six minutes.

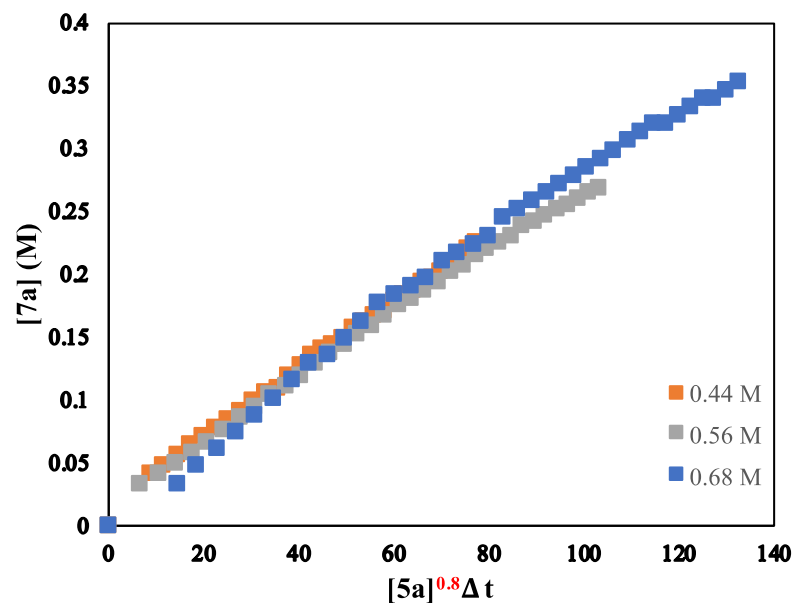

Figure S3. Plot revealing a 0.8-order dependence in oxime.

Table S3. Data for the determination of the reaction order in the oxime component.

\begin{tabular}{|c|c|c|c|c|c|c|c|c|c|c|c|}
\hline \multicolumn{5}{|c|}{$[\mathbf{5 a}]=\mathbf{0 . 4 4} \mathbf{M}$} & \multicolumn{4}{c|}{$[\mathbf{5 a}]=\mathbf{0 . 5 6} \mathbf{M}$} & \multicolumn{4}{|c|}{$[\mathbf{5 a}]=\mathbf{0 . 6 8} \mathbf{M}$} \\
\hline $\begin{array}{c}\text { time } \\
(\mathrm{min})\end{array}$ & $\begin{array}{c}{[\mathbf{7 a}]} \\
(\mathrm{M})\end{array}$ & $\begin{array}{c}{[\mathbf{5 a}]} \\
(\mathrm{M})\end{array}$ & $\begin{array}{c}{[\mathbf{5 a}]^{\beta}} \\
\beta=0.8\end{array}$ & $\begin{array}{c}\text { time } \\
(\mathrm{min})\end{array}$ & $\begin{array}{c}{[\mathbf{7 a}]} \\
(\mathrm{M})\end{array}$ & $\begin{array}{c}{[\mathbf{5 a}]} \\
(\mathrm{M})\end{array}$ & $\begin{array}{c}{[\mathbf{5 a}]^{\beta}} \\
\beta=0.8\end{array}$ & $\begin{array}{c}\text { time } \\
(\mathrm{min})\end{array}$ & $\begin{array}{c}{[\mathbf{7 a}]} \\
(\mathrm{M})\end{array}$ & $\begin{array}{c}{[\mathbf{5 a}]} \\
(\mathrm{M})\end{array}$ & $\begin{array}{c}{[\mathbf{5 a}]^{\beta}} \\
\beta=0.8\end{array}$ \\
\hline 0 & 0 & 0.44 & 0 & 0 & 0 & 0.56 & 0 & 0 & 0 & 0.68 & 0 \\
32 & 0.04224 & 0.39776 & 8.79069 & 20 & 0.03416 & 0.5258 & 6.869 & 20 & 0.034 & 0.646 & 14.396 \\
38 & 0.047872 & 0.392128 & 11.6442 & 26 & 0.042504 & 0.5175 & 10.43 & 26 & 0.0476 & 0.6324 & 18.59 \\
44 & 0.05632 & 0.38368 & 14.4569 & 32 & 0.050232 & 0.5098 & 13.96 & 32 & 0.0612 & 0.6188 & 22.713 \\
50 & 0.06556 & 0.37444 & 17.2183 & 38 & 0.05824 & 0.5018 & 17.43 & 38 & 0.0748 & 0.6052 & 26.764 \\
56 & 0.07216 & 0.36784 & 19.9333 & 44 & 0.0672 & 0.4928 & 20.86 & 44 & 0.0884 & 0.5916 & 30.743 \\
62 & 0.07832 & 0.36168 & 22.611 & 50 & 0.077 & 0.483 & 24.24 & 50 & 0.102 & 0.578 & 34.649 \\
68 & 0.08448 & 0.35552 & 25.2524 & 56 & 0.086128 & 0.4739 & 27.57 & 56 & 0.1156 & 0.5644 & 38.482 \\
74 & 0.09152 & 0.34848 & 27.8549 & 62 & 0.09408 & 0.4659 & 30.85 & 62 & 0.1292 & 0.5508 & 42.242
\end{tabular}




\begin{tabular}{|c|c|c|c|c|c|c|c|c|c|c|c|}
\hline 80 & 0.09944 & 0.34056 & 30.413 & 68 & 0.10416 & 0.4558 & 34.08 & 68 & 0.136 & 0.544 & 45.948 \\
\hline 86 & 0.1056 & 0.3344 & 32.9293 & 74 & 0.11144 & 0.4486 & 37.26 & 74 & 0.1496 & 0.5304 & 49.597 \\
\hline 92 & 0.11 & 0.33 & 35.414 & 80 & 0.1204 & 0.4396 & 40.39 & 80 & 0.1632 & 0.5168 & 53.173 \\
\hline 98 & 0.1188 & 0.3212 & 37.8591 & 86 & 0.12992 & 0.4301 & 43.47 & 86 & 0.1768 & 0.5032 & 56.674 \\
\hline 104 & 0.1276 & 0.3124 & 40.2512 & 92 & 0.1372 & 0.4228 & 46.51 & 92 & 0.1836 & 0.4964 & 60.119 \\
\hline 110 & 0.1364 & 0.3036 & 42.59 & 98 & 0.14448 & 0.4155 & 49.5 & 98 & 0.1904 & 0.4896 & 63.526 \\
\hline 116 & 0.1408 & 0.2992 & 44.8886 & 104 & 0.15344 & 0.4066 & 52.45 & 104 & 0.1972 & 0.4828 & 66.896 \\
\hline 122 & 0.1452 & 0.2948 & 47.1603 & 110 & 0.15904 & 0.401 & 55.35 & 110 & 0.2108 & 0.4692 & 70.209 \\
\hline 128 & 0.1496 & 0.2904 & 49.4051 & 116 & 0.168 & 0.392 & 58.21 & 116 & 0.2176 & 0.4624 & 73.466 \\
\hline 134 & 0.15796 & 0.28204 & 51.6106 & 122 & 0.17584 & 0.3842 & 61.03 & 122 & 0.2244 & 0.4556 & 76.684 \\
\hline 140 & 0.1628 & 0.2772 & 53.7753 & 128 & 0.18144 & 0.3786 & 63.8 & 128 & 0.2312 & 0.4488 & 79.864 \\
\hline 146 & 0.1672 & 0.2728 & 55.9114 & 134 & 0.18816 & 0.3718 & 66.54 & 134 & 0.2448 & 0.4352 & 82.986 \\
\hline 152 & 0.1716 & 0.2684 & 58.0201 & 140 & 0.19488 & 0.3651 & 69.24 & 140 & 0.2516 & 0.4284 & 86.051 \\
\hline 158 & 0.17952 & 0.26048 & 60.0903 & 146 & 0.2016 & 0.3584 & 71.9 & 146 & 0.2584 & 0.4216 & 89.077 \\
\hline 164 & 0.18436 & 0.25564 & 62.1205 & 152 & 0.2072 & 0.3528 & 74.52 & 152 & 0.2652 & 0.4148 & 92.064 \\
\hline 170 & 0.187 & 0.253 & 64.127 & 158 & 0.21504 & 0.345 & 77.11 & 158 & 0.272 & 0.408 & 95.012 \\
\hline 176 & 0.1936 & 0.2464 & 66.1044 & 164 & 0.2212 & 0.3388 & 79.65 & 164 & 0.2788 & 0.4012 & 97.921 \\
\hline 182 & 0.198 & 0.242 & 68.0468 & 170 & 0.22568 & 0.3343 & 82.16 & 170 & 0.2856 & 0.3944 & 100.79 \\
\hline 188 & 0.2024 & 0.2376 & 69.9612 & 176 & 0.23128 & 0.3287 & 84.64 & 176 & 0.2924 & 0.3876 & 103.62 \\
\hline 194 & 0.20856 & 0.23144 & 71.8418 & 182 & 0.238 & 0.322 & 87.09 & 182 & 0.2992 & 0.3808 & 106.41 \\
\hline 200 & 0.2134 & 0.2266 & 73.687 & 188 & 0.24192 & 0.3181 & 89.5 & 188 & 0.306 & 0.374 & 109.16 \\
\hline 206 & 0.22 & 0.22 & 75.4953 & 194 & 0.2464 & 0.3136 & 91.88 & 194 & 0.3128 & 0.3672 & 111.88 \\
\hline 212 & 0.22528 & 0.21472 & 77.265 & 200 & 0.252 & 0.308 & 94.24 & 200 & 0.3196 & 0.3604 & 114.55 \\
\hline- & - & - & - & 206 & 0.25536 & 0.3046 & 96.57 & 206 & 0.3196 & 0.3604 & 117.2 \\
\hline - & - & - & - & 212 & 0.2604 & 0.2996 & 98.87 & 212 & 0.3264 & 0.3536 & 119.83 \\
\hline- & - & - & - & 218 & 0.26544 & 0.2946 & 101.1 & 218 & 0.3332 & 0.3468 & 122.42 \\
\hline- & - & - & - & 224 & 0.2688 & 0.2912 & 103.4 & 224 & 0.34 & 0.34 & 124.98 \\
\hline- & - & - & - & - & - & - & - & 230 & 0.34 & 0.34 & 127.51 \\
\hline- & - & - & - & - & - & - & - & 236 & 0.3468 & 0.3332 & 130.02 \\
\hline- & - & - & - & - & - & - & - & 242 & 0.3536 & 0.3264 & 132.49 \\
\hline
\end{tabular}




\section{Procedure for determining the vinyl ether reaction order dependence}

Using the data obtained from the first experiment (see above), two additional independent reactions were conducted in oven-dried NMR tubes charged with 3,4-dihydro-2H-pyran (6a) $(0.71-$ or $0.95 \mathrm{M})$ to which were diluted in $0.5 \mathrm{~mL} \mathrm{CDCl}_{3}$. Next, acetophenone oxime (5a) (0.68 M) and catalyst $\mathbf{4} \mathbf{e}^{-} \mathbf{B F}_{4}^{-}(5 \mathrm{~mol} \% ; 0.034 \mathrm{M})$ were added to the solutions. Each respective experiment was placed in a ${ }^{1} \mathrm{H}$ NMR $300 \mathrm{MHz}$ spectrometer and allowed to react for $\sim 4$ hours at $40^{\circ} \mathrm{C}$. Reaction progress was tracked by monitoring the disappearance of the signal at $\sim 7.65 \mathrm{ppm}$ for $\mathbf{5} \mathbf{a}$ and appearance of the signal at $\sim 7.71 \mathrm{ppm}$ for product $7 \mathbf{a}$. Data points were recorded every six minutes.

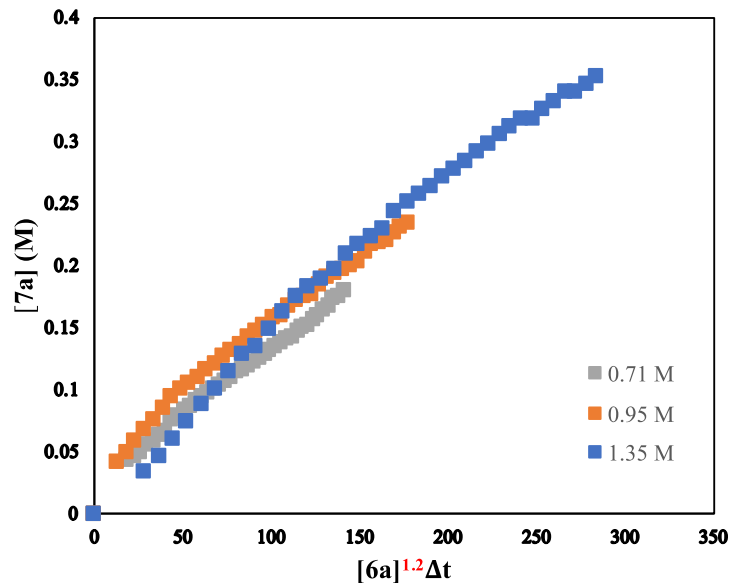

Figure S4. Plot revealing a 1.2-order dependence in vinyl ether.

Table S4. Data for the determination of the reaction order in the vinyl ether component.

\begin{tabular}{|c|c|c|c|c|c|c|c|c|c|c|c|}
\hline \multicolumn{4}{|c|}{$[6 \mathrm{a}]=0.71 \mathrm{M}$} & \multicolumn{4}{|c|}{$[6 \mathrm{a}]=0.95 \mathrm{M}$} & \multicolumn{4}{|c|}{$[6 \mathrm{a}]=1.35 \mathrm{M}$} \\
\hline $\begin{array}{l}\text { time } \\
(\mathrm{min})\end{array}$ & $\begin{array}{l}{[7 \mathbf{7 a}]} \\
(\mathrm{M})\end{array}$ & $\begin{array}{l}{[\mathbf{6 a}]} \\
(\mathrm{M})\end{array}$ & $\begin{array}{c}{[\mathbf{6} \mathbf{a}]^{\beta}} \\
\beta=1.2\end{array}$ & $\begin{array}{l}\text { time } \\
\text { (min) }\end{array}$ & $\begin{array}{r}{[7 \mathrm{a}]} \\
(\mathrm{M})\end{array}$ & $\begin{array}{l}{[\mathbf{6 a}]} \\
(\mathrm{M})\end{array}$ & $\begin{array}{c}{[\mathbf{6 a}]^{\beta}} \\
\beta=1.2\end{array}$ & $\begin{array}{l}\text { time } \\
\text { (min) }\end{array}$ & $\begin{array}{l}{[7 \mathbf{a}]} \\
(\mathrm{M})\end{array}$ & $\begin{array}{l}{[\mathbf{6 a}]} \\
(\mathrm{M})\end{array}$ & $\begin{array}{c}{[\mathbf{6 a}]^{\beta}} \\
\beta=1.2\end{array}$ \\
\hline 0 & 0 & 0.71 & 0 & 0 & 0 & 0.95 & 0 & 0 & 0 & 1.35 & 0 \\
\hline 70 & 0.0442 & 0.6658 & 18.701 & 33 & 0.0425 & 0.9075 & 12.785 & 20 & 0.034 & 1.316 & 28.2375 \\
\hline 76 & 0.04692 & 0.66308 & 22.375 & 39 & 0.05066 & 0.89934 & 18.096 & 26 & 0.0476 & 1.3024 & 36.5276 \\
\hline 82 & 0.050728 & 0.659272 & 26.027 & 45 & 0.059228 & 0.890772 & 23.349 & 32 & 0.0612 & 1.2888 & 44.7144 \\
\hline 88 & 0.05644 & 0.65356 & 29.648 & 51 & 0.06902 & 0.88098 & 28.537 & 38 & 0.0748 & 1.2752 & 52.7982 \\
\hline 94 & 0.059296 & 0.650704 & 33.24 & 57 & 0.07718 & 0.87282 & 33.662 & 44 & 0.0884 & 1.2616 & 60.7793 \\
\hline 100 & 0.063784 & 0.646216 & 36.808 & 63 & 0.08636 & 0.86364 & 38.726 & 50 & 0.102 & 1.248 & 68.6577 \\
\hline 106 & 0.068 & 0.642 & 40.347 & 69 & 0.096016 & 0.853984 & 43.724 & 56 & 0.1156 & 1.2344 & 76.4338 \\
\hline 112 & 0.07616 & 0.63384 & 43.845 & 75 & 0.101456 & 0.848544 & 48.67 & 62 & 0.1292 & 1.2208 & 84.1078 \\
\hline 118 & 0.07956 & 0.63044 & 47.306 & 81 & 0.106216 & 0.843784 & 53.58 & 68 & 0.136 & 1.214 & 91.7053 \\
\hline 124 & 0.085 & 0.625 & 50.737 & 87 & 0.11152 & 0.83848 & 58.455 & 74 & 0.1496 & 1.2004 & 99.2265 \\
\hline 130 & 0.087516 & 0.622484 & 54.142 & 93 & 0.117028 & 0.832972 & 63.293 & 80 & 0.1632 & 1.1868 & 106.646 \\
\hline
\end{tabular}




\begin{tabular}{|c|c|c|c|c|c|c|c|c|c|c|c|}
\hline 136 & 0.09248 & 0.61752 & 57.523 & 99 & 0.122264 & 0.827736 & 68.093 & 86 & 0.1768 & 1.1732 & 113.964 \\
\hline 142 & 0.095064 & 0.614936 & 60.879 & 105 & 0.12716 & 0.82284 & 72.859 & 92 & 0.1836 & 1.1664 & 121.207 \\
\hline 148 & 0.09792 & 0.61208 & 64.218 & 111 & 0.1326 & 0.8174 & 77.588 & 98 & 0.1904 & 1.1596 & 128.399 \\
\hline 154 & 0.101592 & 0.608408 & 67.535 & 117 & 0.13736 & 0.81264 & 82.282 & 104 & 0.1972 & 1.1528 & 135.54 \\
\hline 160 & 0.10472 & 0.60528 & 70.83 & 123 & 0.1428 & 0.8072 & 86.941 & 110 & 0.2108 & 1.1392 & 142.606 \\
\hline 166 & 0.108052 & 0.601948 & 74.104 & 129 & 0.14756 & 0.80244 & 91.565 & 116 & 0.2176 & 1.1324 & 149.597 \\
\hline 172 & 0.11084 & 0.59916 & 77.357 & 135 & 0.153 & 0.797 & 96.153 & 122 & 0.2244 & 1.1256 & 156.537 \\
\hline 178 & 0.11492 & 0.59508 & 80.589 & 141 & 0.15844 & 0.79156 & 100.7 & 128 & 0.2312 & 1.1188 & 163.428 \\
\hline 184 & 0.117232 & 0.592768 & 83.8 & 147 & 0.161092 & 0.788908 & 105.23 & 134 & 0.2448 & 1.1052 & 170.243 \\
\hline 190 & 0.11968 & 0.59032 & 86.996 & 153 & 0.167892 & 0.782108 & 109.72 & 140 & 0.2516 & 1.0984 & 176.983 \\
\hline 196 & 0.12376 & 0.58624 & 90.17 & 159 & 0.1734 & 0.7766 & 114.17 & 146 & 0.2584 & 1.0916 & 183.673 \\
\hline 202 & 0.12614 & 0.58386 & 93.323 & 165 & 0.176052 & 0.773948 & 118.59 & 152 & 0.2652 & 1.0848 & 190.314 \\
\hline 208 & 0.129472 & 0.580528 & 96.458 & 171 & 0.17816 & 0.77184 & 122.99 & 158 & 0.272 & 1.078 & 196.905 \\
\hline 214 & 0.132804 & 0.577196 & 99.572 & 177 & 0.18496 & 0.76504 & 127.37 & 164 & 0.2788 & 1.0712 & 203.446 \\
\hline 220 & 0.135728 & 0.574272 & 102.66 & 183 & 0.19176 & 0.75824 & 131.69 & 170 & 0.2856 & 1.0644 & 209.937 \\
\hline 226 & 0.13872 & 0.57128 & 105.74 & 189 & 0.19448 & 0.75552 & 135.99 & 176 & 0.2924 & 1.0576 & 216.379 \\
\hline 232 & 0.14144 & 0.56856 & 108.79 & 195 & 0.19856 & 0.75144 & 140.26 & 182 & 0.2992 & 1.0508 & 222.771 \\
\hline 238 & 0.14416 & 0.56584 & 111.83 & 201 & 0.2006 & 0.7494 & 144.51 & 188 & 0.306 & 1.044 & 229.114 \\
\hline 244 & 0.14756 & 0.56244 & 114.85 & 207 & 0.20468 & 0.74532 & 148.74 & 194 & 0.3128 & 1.0372 & 235.408 \\
\hline 250 & 0.15164 & 0.55836 & 117.85 & 213 & 0.211208 & 0.738792 & 152.94 & 200 & 0.3196 & 1.0304 & 241.652 \\
\hline 256 & 0.153 & 0.557 & 120.82 & 219 & 0.2176 & 0.7324 & 157.09 & 206 & 0.3196 & 1.0304 & 247.871 \\
\hline 262 & 0.157012 & 0.552988 & 123.78 & 225 & 0.21896 & 0.73104 & 161.21 & 212 & 0.3264 & 1.0236 & 254.066 \\
\hline 268 & 0.1598 & 0.5502 & 126.72 & 231 & 0.221 & 0.729 & 165.33 & 218 & 0.3332 & 1.0168 & 260.212 \\
\hline 274 & 0.16524 & 0.54476 & 129.63 & 237 & 0.2278 & 0.7222 & 169.41 & 224 & 0.34 & 1.01 & 266.308 \\
\hline 280 & 0.16864 & 0.54136 & 132.52 & 243 & 0.23256 & 0.71744 & 173.45 & 230 & 0.34 & 1.01 & 272.381 \\
\hline 286 & 0.17476 & 0.53524 & 135.37 & 249 & 0.23528 & 0.71472 & 177.47 & 236 & 0.3468 & 1.0032 & 278.428 \\
\hline 292 & 0.17612 & 0.53388 & 138.2 & - & - & - & - & 242 & 0.3536 & 0.9964 & 284.427 \\
\hline 298 & 0.1802 & 0.5298 & 141.01 & - & - & - & - & & - & - & . \\
\hline
\end{tabular}




\section{3. ${ }^{1} \mathrm{H}$ NMR titration experiments}

\section{Catalyst dimerization study}

Three stock solutions of catalyst $\mathbf{4} \mathbf{e} \cdot \mathbf{B F}_{4}{ }^{-}(3 \times 32.4 \mathrm{mg}, 0.05 \mathrm{mmol}, 50.1 \mathrm{mM})$ in $\mathrm{CD}_{2} \mathrm{Cl}_{2}(3 \mathrm{x} 1.0$ $\mathrm{mL})$ were prepared in oven-dried $1.0 \mathrm{~mL}$ volumetric flasks. A series of solutions at varying concentrations were then prepared in oven-dried NMR tubes by combining the appropriate amount of stock solution with $\mathrm{CD}_{2} \mathrm{Cl}_{2}$ totalling a volume of $0.5 \mathrm{~mL}$. Each respective NMR sample was then recorded on a ${ }^{1} \mathrm{H}$ NMR $300 \mathrm{MHz}$ spectrometer.

\section{Host-guest binding study involving acetophenone oxime}

A stock solution of catalyst $\mathbf{4 e} \cdot \mathbf{B F}_{4}^{-}(32.4 \mathrm{mg}, 0.05 \mathrm{mmol}, 50.1 \mathrm{mM})$ in $\mathrm{CD}_{2} \mathrm{Cl}_{2}(1.0 \mathrm{~mL})$ and two stock solutions of oxime 5a $(2 \times 6.8 \mathrm{mg}, 0.05 \mathrm{mmol}, 50.3 \mathrm{mM})$ in $\mathrm{CD}_{2} \mathrm{Cl}_{2}(2 \mathrm{x} 1.0 \mathrm{~mL})$ were prepared in oven-dried $1.0 \mathrm{~mL}$ volumetric flasks. A series of solutions containing $5 \mathrm{mM}$ of catalyst $\mathbf{4 e} \cdot \mathbf{B F}_{4}^{-}$were combined with $0.1-6$ equivalents of oxime 5a, and were further diluted in the appropriate amount of $\mathrm{CD}_{2} \mathrm{Cl}_{2}$ in separate oven-dried NMR tubes totalling a volume of $0.5 \mathrm{~mL}$. Each respective NMR sample was then recorded on a ${ }^{1} \mathrm{H}$ NMR $300 \mathrm{MHz}$ spectrometer.

\section{Host-guest binding study involving 3,4-dihydro-2H-pyran}

A stock solution of catalyst $\mathbf{4 e} \cdot \mathbf{B F}_{4}^{-}(32.4 \mathrm{mg}, 0.05 \mathrm{mmol}, 50.1 \mathrm{mM})$ in $\mathrm{CD}_{2} \mathrm{Cl}_{2}(1.0 \mathrm{~mL})$ was prepared in an oven-dried $1.0 \mathrm{~mL}$ volumetric flask. A solution containing $5 \mathrm{mM}$ of catalyst $\mathbf{4 e} \cdot \mathbf{B F}_{\mathbf{4}}{ }^{-}$ $\left(0.5 \mathrm{~mL}\right.$ of $\left.\mathrm{CD}_{2} \mathrm{Cl}_{2}\right)$ were titrated in 5-230 equivalents of 3,4-dihydro- $2 \mathrm{H}$-pyran (6a) sequentially in an oven-dried NMR tube. Each respective NMR titration sample was then recorded on a ${ }^{1} \mathrm{H}$ NMR $300 \mathrm{MHz}$ spectrometer. 


\section{Synthetic procedures and characterization data of catalysts and products}

\section{Synthetic procedures and characterization data of catalysts}

$\boldsymbol{N}$-(2,3-Bis(diisopropylamino)cyclopropenium)- $\boldsymbol{N}^{\prime}$-phenyl-urea $(\mathbf{4 c}) \cdot \mathbf{B F}_{4}^{-} \cdot$ A solution of phenyl isocyanate $(123 \mathrm{mg}, 1.0 \mathrm{mmol}, 1.0$ equiv.) in DCM (1.00 mL) was added dropwise to a $10.0 \mathrm{~mL}$ flame-dried round-bottom flask containing a solution of 2,3 bis(diisopropylamino)cyclopropenimine (1) $(260 \mathrm{mg}, 1.0 \mathrm{mmol}, 1.0$ equiv.) in DCM (3.00 mL). The solution was vigorously stirred for 1 hour at room temperature under an inert atmosphere. Following this event, the solution was washed with $3 \mathrm{M} \mathrm{HCl}$ until disappearance of $\mathbf{1}$, as visualized via TLC. Next, the organic layer was first washed with saturated bicarbonate $(1 \times 30 \mathrm{~mL})$ followed by a wash using a $3 \mathrm{M}$ solution of $50 \% \mathrm{HBF}_{4(\mathrm{aq})}(1 \times 30.0 \mathrm{~mL})$. The organic layer was then dried over $\mathrm{MgSO}_{4}$ and concentrated in vacuo to afford a viscous, colorless oil. The oil was then triturated with diethyl ether $(1 \times 10.0 \mathrm{~mL})$ to furnish urea $4 \mathbf{c} \cdot \mathrm{BF}_{4}{ }^{-}$as a white solid $(225 \mathrm{mg}, 47 \%)$. Mp: 171$173{ }^{\circ} \mathrm{C} ;{ }^{1} \mathrm{H}$ NMR $\left(300 \mathrm{MHz}, \mathrm{CDCl}_{3}, 25{ }^{\circ} \mathrm{C}\right): \delta=1.38-1.40(\mathrm{~d}, J=6.8 \mathrm{~Hz} ; 24 \mathrm{H}), 4.11-4.24(\mathrm{~m}$, 4H), 7.06-7.11 (t, $J=7.4 \mathrm{~Hz}$; 1H), 7.29-7.34 (overlapping signals; 2H), 7.60-7.63 (d, $J=7.8 \mathrm{~Hz}$; $2 \mathrm{H}), 8.48(\mathrm{~s}, 1 \mathrm{H}), 8.91(\mathrm{~s}, 1 \mathrm{H}) ;{ }^{13} \mathrm{C}\left\{{ }^{1} \mathrm{H}\right\} \mathrm{NMR}\left(75.5 \mathrm{MHz}, \mathrm{CDCl}_{3}, 25{ }^{\circ} \mathrm{C}\right): \delta=21.7,51.0,106.2$, $119.6(\mathrm{~d}, J=6.6 \mathrm{~Hz}), 123.8,128.8,137.9,149.8 ;{ }^{19} \mathrm{~F}\left\{{ }^{1} \mathrm{H}\right\} \mathrm{NMR}\left(292.4 \mathrm{MHz}, \mathrm{CDCl}_{3}, 25{ }^{\circ} \mathrm{C}\right) \delta=$ -148.8; ${ }^{11} \mathrm{~B}\left\{{ }^{1} \mathrm{H}\right\}$ NMR (96.3 MHz, $\left.\mathrm{CDCl}_{3}, 25{ }^{\circ} \mathrm{C}\right) \delta=-0.8$; HRMS (FAB-DFS) $m / z$ : [M - BF $]^{+}$ Calcd for $\mathrm{C}_{22} \mathrm{H}_{35} \mathrm{~N}_{4} \mathrm{O}^{+} 371.2794$; Found 371.2802.

3-((2,3-Bis(diisopropylamino)cyclopropenimine-4-(cyclopentyloxy)cyclobut-3-ene-1,2-dione (3). To a flame-dried $50.0 \mathrm{~mL}$ round-bottom flask charged with 1 (3.62 g, 14.4 mmol, 3.0 equiv.) and 3,4-bis(cyclopentyloxy)cyclobut-3-ene-1,2-dione (2) (1.20 g, $4.80 \mathrm{mmol}, 1.0$ equiv.) was added DCM $(15.0 \mathrm{~mL})$. The solution was vigorously stirred for 1 hour at room temperature under an inert atmosphere. Next, the solution was washed with $3 \mathrm{M} \mathrm{HCl}$ until disappearance of 2, as visualized via TLC. The combined organic layers were dried over $\mathrm{MgSO}_{4}$ and concentrated in vacuo. The resulting residue was then triturated with ice-cold hexane to afford $\mathbf{3}$ as an off-white solid. (1.93 g, 97\%). Mp: $171-173{ }^{\circ} \mathrm{C}$; ${ }^{1} \mathrm{H}$ NMR (300 MHz, $\left.\mathrm{CDCl}_{3}, 2{ }^{\circ} \mathrm{C}\right): \delta=1.39-1.41(\mathrm{~d}, J=$ $6.0 \mathrm{~Hz} ; 24 \mathrm{H}), 1.59-1.72(\mathrm{~m}, 2 \mathrm{H}), 1.84-1.97(\mathrm{~m}, 2 \mathrm{H}), 2.02-2.08(\mathrm{~m}, 4 \mathrm{H}), 3.82-3.95(\mathrm{~m}, 4 \mathrm{H})$, 5.74-5.79 (m, 1H); ${ }^{13} \mathrm{C}\left\{{ }^{1} \mathrm{H}\right\} \operatorname{NMR}\left(75.5 \mathrm{MHz}, \mathrm{CDCl}_{3}, 25{ }^{\circ} \mathrm{C}\right): \delta=21.9,23.7,33.7,50.7,85.0$, 123.0, 178.2, 182.5, 185.7, 197.0; HRMS (EI-DFS) $m / z:[\mathrm{M}+\mathrm{H}]^{+}$Calcd for $\mathrm{C}_{24} \mathrm{H}_{37} \mathrm{O}_{3} \mathrm{~N}_{3}$ 415.2835; Found 415.2826.

\section{3-((2,3-Bis(diisopropylamino)cyclopropenium-4-(phenylamino)cyclobut-3-ene-1,2-dione}

(4d) $\mathbf{B F}_{4}{ }^{-}$. To a flame-dried $25.0 \mathrm{~mL}$ round-bottom flask charged with $\mathbf{3}(300 \mathrm{mg}, 0.722 \mathrm{mmol}$, 1.0 equiv) was added DCM (4.0 mL). Aniline ( $0.65 \mathrm{~mL}, 7.12 \mathrm{mmol}, 10$ equiv) was then slowly added to the solution. Next, the flask was equipped with a reflux condenser and heated in an oil bath to reflux, whilst stirred under an inert atmosphere for 7 days. The resulting solution was washed with $3 \mathrm{M} \mathrm{HCl}(2 \times 50 \mathrm{~mL})$, and the aqueous layer extracted with DCM $(3 \times 5 \mathrm{~mL})$. Then, the combined organic fractions were washed with saturated bicarbonate $(1 \times 20 \mathrm{~mL})$ followed by $3 \mathrm{M} \mathrm{HBF}_{4}(1 \times 20.0 \mathrm{~mL})$, dried over $\mathrm{MgSO}_{4}$ and concentrated down to a dark yellow residue. The residue was then subjected to vacuum filtration, where it was washed multiple times with ethyl acetate until a light yellow powder was obtained. (136 mg, 37\%). Mp: $213-215{ }^{\circ} \mathrm{C} ;{ }^{1} \mathrm{H}$ NMR $(300$ $\left.\mathrm{MHz} \mathrm{CDCl}_{3}, 25^{\circ} \mathrm{C}\right): \delta=1.41-1.43(\mathrm{~d}, J=6.9 \mathrm{~Hz} ; 24 \mathrm{H}), 3.90-3.99(\mathrm{~m}, 4 \mathrm{H}), 7.14-7.19$ (t, $J=7.4$ $\mathrm{Hz} ; 1 \mathrm{H}), 7.36-7.41$ (t, $J=8.4 \mathrm{~Hz} ; 2 \mathrm{H}), 7.61-7.64$ (d, $J=7.8 \mathrm{~Hz} ; 2 \mathrm{H}), 8.88$ (s, 1H), 9.02 (s, 1H); 
${ }^{13} \mathrm{C}\left\{{ }^{1} \mathrm{H}\right\} \mathrm{NMR}\left(75.5 \mathrm{MHz}, \mathrm{CDCl}_{3}, 25^{\circ} \mathrm{C}\right): \delta=21.7,52.2,104.3,119.9,124.6,124.9,129.2,137.4$, 161.7, 169.4, 182.8, 184.0; ${ }^{19} \mathrm{~F}\left\{{ }^{1} \mathrm{H}\right\} \operatorname{NMR}\left(292.4 \mathrm{MHz}, \mathrm{CDCl}_{3}, 25{ }^{\circ} \mathrm{C}\right) \delta=-147.4 ;{ }^{11} \mathrm{~B}\left\{{ }^{1} \mathrm{H}\right\} \mathrm{NMR}$ (96.3 $\mathrm{MHz}, \mathrm{CDCl}_{3}, 25{ }^{\circ} \mathrm{C}$ ) $\delta=-0.8$; HRMS (FAB-DFS) $m / z$ : $\left[\mathrm{M}-\mathrm{BF}_{4}\right]^{+}$Calcd for $\mathrm{C}_{25} \mathrm{H}_{35} \mathrm{O}_{2} \mathrm{~N}_{4}{ }^{+}$ 423.2744; Found 423.2749.

\section{3-((2,3-Bis(diisopropylamino)cyclopropenium-4-((3,5-}

bis(trifluoromethyl)phenyl)amino)cyclobut-3-ene-1,2-dione (4e) $\mathbf{B F}_{4}^{-}$. To a flame-dried 50.0 $\mathrm{mL}$ round-bottom flask charged with 3 ( $1.3 \mathrm{~g}, 3.13 \mathrm{mmol}, 1.0$ equiv) was added DCM (15.0 $\mathrm{mL})$. 3,5-Bis(trifluoromethyl)aniline $(2.70 \mathrm{~mL}, 17.3 \mathrm{mmol}, 5.5$ equiv) was then slowly added to the solution. Next, the flask was equipped with a reflux condenser and heated in an oil bath to reflux, whilst stirred under an inert atmosphere for 7 days. The resulting black solution was washed with $3 \mathrm{M} \mathrm{HCl}(2 \times 200 \mathrm{~mL})$, and the aqueous layer extracted with DCM (3 x $10 \mathrm{~mL})$. Then, the combined organic fractions were washed with saturated bicarbonate $(1 \times 50 \mathrm{~mL})$ followed by $3 \mathrm{M}$ $\mathrm{HBF}_{4}(1 \times 50.0 \mathrm{~mL})$, dried over $\mathrm{MgSO}_{4}$ and concentrated down to a dark red residue. The residue was then subjected to vacuum filtration, wherein it was washed multiple times with ethyl acetate until an off-white powder was obtained. (1.02 g, 50\%). Mp: 230-232 ${ }^{\circ} \mathrm{C} ;{ }^{1} \mathrm{H}$ NMR (300 MHz, $\left.\mathrm{CDCl}_{3}, 25^{\circ} \mathrm{C}\right): \delta=1.41-1.43(\mathrm{~d}, J=6.8 \mathrm{~Hz} ; 24 \mathrm{H}), 3.87-3.99(\mathrm{~m}, 4 \mathrm{H}), 7.63(\mathrm{~s}, 1 \mathrm{H}), 8.14(\mathrm{~s}, 2 \mathrm{H})$, $9.01(\mathrm{~s}, 1 \mathrm{H}), 9.28(\mathrm{~s}, 1 \mathrm{H}) ;{ }^{13} \mathrm{C}\left\{{ }^{1} \mathrm{H}\right\} \mathrm{NMR}\left(75.5 \mathrm{MHz}, \mathrm{CDCl}_{3}, 25{ }^{\circ} \mathrm{C}\right): \delta=21.6,52.3,103.6,117.6$, $119.7,121.2,124.8,125.4,132.5,132.9,139.2,163.1,168.6,183.1,183.3 ;{ }^{19} \mathrm{~F}\left\{{ }^{1} \mathrm{H}\right\} \mathrm{NMR}(292.4$ $\left.\mathrm{MHz}, \mathrm{CDCl}_{3}, 25{ }^{\circ} \mathrm{C}\right) \delta=-146.5,-63.1 ;{ }^{11} \mathrm{~B}\left\{{ }^{1} \mathrm{H}\right\} \mathrm{NMR}$ (96.3 MHz, $\left.\mathrm{CDCl}_{3}, 25{ }^{\circ} \mathrm{C}\right) \delta=-0.8$; HRMS (FAB-DFS) $m / z$ : $\left[\mathrm{M}-\mathrm{BF}_{4}\right]^{+}$Calcd for $\mathrm{C}_{27} \mathrm{H}_{33} \mathrm{O}_{2} \mathrm{~N}_{4} \mathrm{~F}_{6}{ }^{+}$559.2491; Found 559.2512.

\section{Characterization data of oxime products (S1-3).}

(Z/E)-3-Methyl-2-((Z)-pent-2-en-1-yl)cyclopent-2-enone oxime (S1). (0.97 g, 89\%), yellowbrown oil. ${ }^{1} \mathrm{H}$ NMR $\left(300 \mathrm{MHz}, \mathrm{CDCl}_{3}, 25{ }^{\circ} \mathrm{C}\right) \delta=1.00(\mathrm{~m}, 3 \mathrm{H}$, major and minor), 1.89 (s, 3H, major and minor), 2.08-2.20 (m, 2H, major and minor), 2.41-2.45 (m, 2H, major and minor), 2.66-2.70 (m, 2H, major and minor), 2.97-2.99 (m, 2H, major and minor), 5.31-5.40 (m, 2H, major and minor), $8.52(\mathrm{~s}, 1 \mathrm{H}) ;{ }^{13} \mathrm{C}\left\{{ }^{1} \mathrm{H}\right\} \mathrm{NMR}\left(75.5 \mathrm{MHz}, \mathrm{CDCl}_{3}, 25{ }^{\circ} \mathrm{C}\right): \delta=14.2,15.3,21.6$, 22.6, 24.5, 34.3, 125.1, 125.6, 132.0, 133.0, 151.6, 168.2; HRMS (EI-DFS) $m / z:[\mathrm{M}+\mathrm{H}]^{+}$Calcd for $\mathrm{C}_{11} \mathrm{H}_{17} \mathrm{NO}$ 179.1310; Found 179.1301.

(Z/E)-3-(4-(tert-Butyl)phenyl)-2-methylpropanal oxime (S2). (0.98 g, 92\%), yellow oil. ${ }^{1} \mathrm{H}$ NMR $\left(300 \mathrm{MHz}, \mathrm{CDCl}_{3}, 25^{\circ} \mathrm{C}\right): \delta=1.07-1.12(\mathrm{t}, J=9.2 \mathrm{~Hz} ; 3 \mathrm{H}$, major and minor), 1.34, (s, 9H, major and minor), 2.58-2.87 (m, 3H, major and minor), $6.62-6.64(\mathrm{~d}, J=7.4 \mathrm{~Hz} ; 1 \mathrm{H}$, minor $), 7.11-7.16$ (t, $J=9.2 \mathrm{~Hz} ; 2 \mathrm{H}$, major and minor), 7.32-7.35 (d, $J=8.2 \mathrm{~Hz}, 2 \mathrm{H}$, major and minor), 7.42-7.44 $(\mathrm{d}, J=5.9 \mathrm{~Hz} ; 1 \mathrm{H}$, major $) ;{ }^{13} \mathrm{C}\left\{{ }^{1} \mathrm{H}\right\} \mathrm{NMR}\left(75.5 \mathrm{MHz}, \mathrm{CDCl}_{3}, 25{ }^{\circ} \mathrm{C}\right): \delta=16.8,17.3,31.1,31.4$, 34.4, 36.1, 39.6, 40.4, 125.2-125.3 (d, $J=4.5 \mathrm{~Hz}), 128.7-128.8$ (d, $J=6.8 \mathrm{~Hz}$ ), 136.0, 149.1, 155.9, 156.7; HRMS (EI-DFS) $m / z$ : $[\mathrm{M}+\mathrm{H}]^{+}$Calcd for $\mathrm{C}_{14} \mathrm{H}_{21} \mathrm{NO} 219.1623$; Found 219.1615.

(Z/E)-4-(4-Methylpent-3-en-1-yl)cyclohex-3-enecarbaldehyde oxime (S3). (0.58 g, 54\%), clear oil. ${ }^{1} \mathrm{H}$ NMR $\left(300 \mathrm{MHz}, \mathrm{CDCl}_{3}, 25^{\circ} \mathrm{C}\right): \delta=1.62$ (s, $3 \mathrm{H}$, major and minor), 1.70 (s, $3 \mathrm{H}$, major and minor $), 1.83-2.23(\mathrm{~m}, 10 \mathrm{H}$, major and minor), 2.42-2.54 (m, 1H, major $), 3.18-3.27(\mathrm{~m}, 1 \mathrm{H}$, minor), 5.09-5.13 (m, 1H, major and minor $), 5.41(\mathrm{~m}, 1 \mathrm{H}$, major and minor $), 6.64-6.66(\mathrm{~d}, \mathrm{~J}=$ $7.2 \mathrm{~Hz} ; 1 \mathrm{H}$, minor $), 7.41-7.43(\mathrm{~d}, J=6.2 \mathrm{~Hz} ; 1 \mathrm{H}$, major $), 8.78(\mathrm{~s}, 1 \mathrm{H}$, major and minor $) ;{ }^{13} \mathrm{C}\left\{{ }^{1} \mathrm{H}\right\}$ NMR (75.5 MHz, $\left.\mathrm{CDCl}_{3}, 25^{\circ} \mathrm{C}\right): \delta=17.7,23.8,24.2,25.2,25.6,25.7,26.4,26.6,26.9,27.3$, 
28.2, 28.8, 29.8, $30.231 .1,31.834 .5,35.0,37.7(\mathrm{~d}, J=8.4 \mathrm{~Hz}), 118.8,120.6(\mathrm{~d}, J=5.6 \mathrm{~Hz}), 124.2$, 131.5, 135.8, 137.7, 155.4, 156.2; HRMS (EI-DFS) $m / z$ : $[\mathrm{M}+\mathrm{H}]^{+}$Calcd for $\mathrm{C}_{13} \mathrm{H}_{21} \mathrm{NO} 207.1623$; Found 207.1617.

\section{Representative procedure for squaramide-catalyzed oxime ether formation.}

To an oven-dried $5.0 \mathrm{~mL}$ round-bottom flask charged with vinyl ether $(1.03 \mathrm{mmol})$ was added $\operatorname{DCM}(0.5 \mathrm{~mL})$. The respective oxime $(0.52 \mathrm{mmol})$ and catalyst $4 \mathrm{e} \cdot \mathrm{BF}_{4}^{-}(17 \mathrm{mg}, 5 \mathrm{~mol} \%)$ were then added to the solution. Next, the flask was equipped with a reflux condenser and heated in an oil bath to reflux, whilst stirred under an inert atmosphere. Reaction progress was monitored via TLC. After removal of the solvent, the crude material was subjected to flash chromatography using a hexanes/ethyl acetate solvent system to furnish the respective oxime ether product.

\section{Characterization data of oxime ether products (7a-7ad).}

(Z/E)-Acetophenone $O$-tetrahydro-2H-pyran-2-yl oxime (7a). (179 mg, 82\%, 1:3 Z/E), clear oil. ${ }^{1} \mathrm{H}$ NMR (300 MHz, $\left.\mathrm{CDCl}_{3}, 25^{\circ} \mathrm{C}\right): \delta=1.53-1.95(\mathrm{~m}, 6 \mathrm{H}, E$ and $Z) ; 2.33$ (s, 3H, $E$ and $Z$ ), 3.50-3.57 $(\mathrm{m}, 1 \mathrm{H}, Z), 3.63-3.70(\mathrm{~m}, 1 \mathrm{H}, E), 3.86-3.91(\mathrm{~m}, 1 \mathrm{H}, Z), 3.93-4.00(\mathrm{~m}, 1 \mathrm{H}, E), 4.97-5.00(\mathrm{~m}, 1 \mathrm{H}$, $Z), 5.43-5.45(\mathrm{~m}, 1 \mathrm{H}, E), 7.36-7.38(\mathrm{~m}, 3 \mathrm{H}, E$ and $Z), 7.68-7.72(\mathrm{~m}, 2 \mathrm{H}, E$ and $Z),{ }^{13} \mathrm{C}\left\{{ }^{1} \mathrm{H}\right\} \mathrm{NMR}$ $\left(75.5 \mathrm{MHz}, \mathrm{CDCl}_{3}, 25^{\circ} \mathrm{C}\right): \delta=13.0,19.8-20.1(\mathrm{~d}, J=22.6 \mathrm{~Hz}), 25.3-25.4(\mathrm{~d}, J=7.6 \mathrm{~Hz}), 29.1$, 30.7, 63.1, 94.6, 101.1, 126.3, 129.2, 136.5, 156.3; HRMS (EI-DFS) $m / z$ : $[\mathrm{M}+\mathrm{H}]^{+}$Calcd for $\mathrm{C}_{13} \mathrm{H}_{17} \mathrm{NO}_{2} 219.1259$; Found 219.1252.

(E)-Acetophenone $O$-tetrahydro-2H-pyran-2-yl oxime (7a). (179 mg, 82\%, 1:19 Z/E), clear oil. ${ }^{1} \mathrm{H}$ NMR $\left(300 \mathrm{MHz}, \mathrm{CDCl}_{3}, 25^{\circ} \mathrm{C}\right): \delta=1.62-1.93(\mathrm{~m}, 6 \mathrm{H}), 2.34(\mathrm{~s}, 3 \mathrm{H}), 3.63-3.71(\mathrm{~m}, 1 \mathrm{H}), 3.93-$ $4.01(\mathrm{~m}, 1 \mathrm{H}), 5.43-5.45(\mathrm{~m}, 1 \mathrm{H}), 7.36-7.38(\mathrm{~m}, 3 \mathrm{H}), 7.68-7.72(\mathrm{~m}, 2 \mathrm{H}) ;{ }^{13} \mathrm{C}\left\{{ }^{1} \mathrm{H}\right\}$ NMR $(75.5$ $\left.\mathrm{MHz}, \mathrm{CDCl}_{3}, 25^{\circ} \mathrm{C}\right): \delta=13.0,20.1,25.3,29.2,63.2,101.1,126.4,128.3,129.2,136.5,156.3$.

(E)-1-(4-(Trifluoromethyl)phenyl)ethenone $O$-tetrahydro-2H-pyran-2-yl oxime (7b). (113 mg, $76 \%, 1: 19 \mathrm{Z} / \mathrm{E})$, clear oil. ${ }^{1} \mathrm{H}$ NMR $\left(300 \mathrm{MHz}, \mathrm{CDCl}_{3}, 25^{\circ} \mathrm{C}\right): \delta=1.64-1.92(\mathrm{~m}, 6 \mathrm{H}), 2.34(\mathrm{~s}, 3 \mathrm{H})$, 3.65-3.71 (m, 1H), 3.92-3.99 (m, 1H), 5.42-5.44 (m, 1H), 7.61-7.64 (d, J=8.2 Hz; 2H), 7.80$7.83(\mathrm{~d}, J=8.1 \mathrm{~Hz} ; 2 \mathrm{H}) ;{ }^{13} \mathrm{C}\left\{{ }^{1} \mathrm{H}\right\} \operatorname{NMR}\left(75.5 \mathrm{MHz}, \mathrm{CDCl}_{3}, 25^{\circ} \mathrm{C}\right) \delta=12.8,19.9,25.2,29.0,63.1$, 101.3, 125.2, 125.8, 126.6, 139.8, 154.9; HRMS (EI-DFS) $m / z:[\mathrm{M}+\mathrm{H}]^{+}$Calcd for $\mathrm{C}_{14} \mathrm{H}_{16} \mathrm{~F}_{3} \mathrm{NO}_{2}$ 287.1133; Found 287.1129.

(E)-1-(4-(Trifluoromethoxy)phenyl)ethenone $O$-tetrahydro-2 $H$-pyran-2-yl oxime (7c) (96 mg, $61 \%, 1: 19 \mathrm{Z} / E)$, yellow oil. ${ }^{1} \mathrm{H}$ NMR $\left(300 \mathrm{MHz}, \mathrm{CDCl}_{3}, 25^{\circ} \mathrm{C}\right): \delta=1.62-1.92(\mathrm{~m}, 6 \mathrm{H}), 2.32(\mathrm{~s}$, $3 \mathrm{H}), 3.64-3.70(\mathrm{~m}, 1 \mathrm{H}), 3.92-3.99(\mathrm{~m}, 1 \mathrm{H}), 5.41-5.44(\mathrm{~m}, 1 \mathrm{H}), 7.20-7.23(\mathrm{~d}, J=8.2 \mathrm{~Hz} ; 2 \mathrm{H})$, 7.72-7.75 (d, $J=8.9 \mathrm{~Hz} ; 2 \mathrm{H}) ;{ }^{13} \mathrm{C}\left\{{ }^{1} \mathrm{H}\right\} \operatorname{NMR}\left(75.5 \mathrm{MHz}, \mathrm{CDCl}_{3}, 25{ }^{\circ} \mathrm{C}\right): \delta=12.9,20.0,25.2$, 29.0, 63.1, 101.2, 118.7, 120.6, 122.1, 127.8, 135.1, 149.9, 155.0; HRMS (EI-DFS) $m / z:[\mathrm{M}+\mathrm{H}]^{+}$ Calcd for $\mathrm{C}_{14} \mathrm{H}_{16} \mathrm{~F}_{3} \mathrm{NO}_{3}$ 303.1082; Found 303.1078.

(E)-Benzaldehyde $O$-tetrahydro- $2 H$-pyran-2-yl oxime (7d). $(52 \mathrm{mg}, 98 \%, 1: 1 \mathrm{Z/E})$, clear oil. ${ }^{1} \mathrm{H}$ NMR $\left(300 \mathrm{MHz}, \mathrm{CDCl}_{3}, 25^{\circ} \mathrm{C}\right): \delta=1.58-1.93(\mathrm{~m}, 6 \mathrm{H}), 3.62-3.70(\mathrm{~m}, 1 \mathrm{H}), 3.94-4.01(\mathrm{~m}, 1 \mathrm{H})$, 5.40-5.42 (m, 1H), 7.37-7.39 (m, 3H), 7.63-7.66 (m, 2H) $8.21(\mathrm{~s}, 1 \mathrm{H}) ;{ }^{13} \mathrm{C}\left\{{ }^{1} \mathrm{H}\right\} \mathrm{NMR}(75.5 \mathrm{MHz}$, 
$\left.\mathrm{CDCl}_{3}, 25^{\circ} \mathrm{C}\right) \delta=19.8,25.2,28.9,62.9,101.0,127.4,128.6,130.0,132.0,150.4$; HRMS (EIDFS) $m / z:[\mathrm{M}+\mathrm{H}]^{+}$Calcd for $\mathrm{C}_{12} \mathrm{H}_{15} \mathrm{NO}_{2}$ 205.1103; Found 205.1095.

(Z)-Benzaldehyde $O$-tetrahydro-2H-pyran-2-yl oxime (7d'). (51 mg, 96\%), clear oil. ${ }^{1} \mathrm{H}$ NMR $\left(300 \mathrm{MHz}, \mathrm{CDCl}_{3}, 25^{\circ} \mathrm{C}\right): \delta=1.60-1.91(\mathrm{~m}, 6 \mathrm{H}), 3.65-3.72(\mathrm{~m}, 1 \mathrm{H}), 3.89-3.97(\mathrm{~m}, 1 \mathrm{H}), 5.42-$ $5.44(\mathrm{~m}, 1 \mathrm{H}), 7.43-7.47$ (overlapping signals, $4 \mathrm{H}), 7.93-7.96(\mathrm{~m}, 2 \mathrm{H}) ;{ }^{13} \mathrm{C}\left\{{ }^{1} \mathrm{H}\right\} \mathrm{NMR}(75.5 \mathrm{MHz}$, $\left.\mathrm{CDCl}_{3}, 25^{\circ} \mathrm{C}\right) \delta=19.7,25.2,28.7,62.6,101.4,127.4,128.5,130.2,130.6,131.0,147.4$.

(E)-4-Methylbenzaldehyde $O$-tetrahydro-2H-pyran-2-yl oxime (7e). (55 mg, 96\%, 1:1 Z/E), clear oil. ${ }^{1} \mathrm{H}$ NMR $\left(300 \mathrm{MHz}, \mathrm{CDCl}_{3}, 25^{\circ} \mathrm{C}\right): \delta=1.59-1.93(\mathrm{~m}, 6 \mathrm{H}), 2.38(\mathrm{~s}, 3 \mathrm{H}), 3.63-3.70(\mathrm{~m}, 1 \mathrm{H})$, 3.94-4.02 (m, 1H), 5.38-5.40 (m, 1H), 7.17-7.20 (d, J=8.0 Hz, 2H), 7.52-7.55 (d, J=8.1 Hz; 2H), $8.19(\mathrm{~s}, 1 \mathrm{H}) ;{ }^{13} \mathrm{C}\left\{{ }^{1} \mathrm{H}\right\} \mathrm{NMR}\left(75.5 \mathrm{MHz}, \mathrm{CDCl}_{3}, 25{ }^{\circ} \mathrm{C}\right): \delta=19.9,21.5,25.2,29.0,63.0$, 101.0, 127.3, 129.3, 140.3, 150.5; HRMS (EI-DFS) $m / z$ : $[\mathrm{M}+\mathrm{H}]^{+}$Calcd for $\mathrm{C}_{13} \mathrm{H}_{17} \mathrm{NO}_{2} 219.1259$; Found 219.1252.

(Z)-4-Methylbenzaldehyde $O$-tetrahydro-2H-pyran-2-yl oxime (7e'). (50 mg, 88\%), clear oil. ${ }^{1} \mathrm{H}$ NMR (300 MHz, CDCl3, $\left.25^{\circ} \mathrm{C}\right): \delta=1.57-1.91(\mathrm{~m}, 6 \mathrm{H}), 2.41(\mathrm{~s}, 3 \mathrm{H}), 3.64-3.71(\mathrm{~m}, 1 \mathrm{H}), 3.89-$ $3.97(\mathrm{~m}, 1 \mathrm{H}), 5.41-5.43(\mathrm{~m}, 1 \mathrm{H}), 7.24-7.28(\mathrm{~d}, J=8.1 \mathrm{~Hz} ; 2 \mathrm{H}), 7.41(\mathrm{~s}, 1 \mathrm{H}), 7.83-7.86(\mathrm{~d}, J=$ $8.2 \mathrm{~Hz} ; 2 \mathrm{H}) ;{ }^{13} \mathrm{C}\left\{{ }^{1} \mathrm{H}\right\} \mathrm{NMR}\left(75.5 \mathrm{MHz}, \mathrm{CDCl}_{3}, 25^{\circ} \mathrm{C}\right): \delta=19.7,21.5,25.2,28.7,62.6,63.4$, 98.5, 101.3, 98.5, 101.3, 128.0, 129.2, 131.1, 140.6, 147.4 .

(E)-4-Methoxybenzaldehyde $O$-tetrahydro-2H-pyran-2-yl oxime (7f). (58 mg, 95\%, 1:1.2 Z/E), clear oil. ${ }^{1} \mathrm{H}$ NMR $\left(300 \mathrm{MHz}, \mathrm{CDCl}_{3}, 25^{\circ} \mathrm{C}\right): \delta=1.57-1.93(\mathrm{~m}, 6 \mathrm{H}) 3.62-3.69(\mathrm{~m}, 1 \mathrm{H}), 3.84(\mathrm{~s}$, $3 \mathrm{H}), 4.01-4.02(\mathrm{~m}, 1 \mathrm{H}), 5.36-5.39(\mathrm{~m}, 1 \mathrm{H}), 6.88-6.92(\mathrm{~d}, J=8.8 \mathrm{~Hz} ; 2 \mathrm{H}), 7.57-7.61$ (d, $J=8.8$ $\mathrm{Hz} ; 2 \mathrm{H}), 8.17(\mathrm{~s}, 1 \mathrm{H}) ;{ }^{13} \mathrm{C}\left\{{ }^{1} \mathrm{H}\right\} \mathrm{NMR}\left(75.5 \mathrm{MHz}, \mathrm{CDCl}_{3}, 25{ }^{\circ} \mathrm{C}\right) \delta=19.9,25.2,29.0,55.3,63.0$, 100.9, 114.1, 124.6, 128.9, 150.1, 161.1; HRMS (EI-DFS) $m / z$ : $[\mathrm{M}+\mathrm{H}]^{+}$Calcd for $\mathrm{C}_{13} \mathrm{H}_{17} \mathrm{NO}_{3}$ 235.1208; Found 235.1205.

(Z)-4-Methoxybenzaldehyde $O$-tetrahydro-2H-pyran-2-yl oxime (7f'). (35 mg, 57\%), clear oil. ${ }^{1} \mathrm{H}$ NMR (300 MHz, $\left.\mathrm{CDCl}_{3}, 25^{\circ} \mathrm{C}\right): \delta=1.61-1.95(\mathrm{~m}, 6 \mathrm{H}), 3.65-3.72(\mathrm{~m}, 1 \mathrm{H}), 3.87(\mathrm{~s}, 3 \mathrm{H}), 3.89-$ $3.97(\mathrm{~m}, 1 \mathrm{H}), 5.40-5.42(\mathrm{~m}, 1 \mathrm{H}),, 6.94-6.97(\mathrm{~d}, J=8.9 \mathrm{~Hz} ; 2 \mathrm{H}) 7.37(\mathrm{~s}, 1 \mathrm{H}), 7.91-7.94(\mathrm{~d}, J=$ $8.9 \mathrm{~Hz} ; 2 \mathrm{H}) ;{ }^{13} \mathrm{C}\left\{{ }^{1} \mathrm{H}\right\} \mathrm{NMR}\left(75.5 \mathrm{MHz}, \mathrm{CDCl}_{3}, 25{ }^{\circ} \mathrm{C}\right) \delta=19.8,25.2,28.8,55.3,62.7,101.3$, $113.8,123.7,133.0,147.0,160.8$.

(E)-6-Bromobenzo[d][1,3]dioxole-5-carabaldehyde $O$-tetrahydro-2H-pyran-2-yl oxime (7g). (119 mg, 70\%, E), white solid. Mp: 92-94 ${ }^{\circ} \mathrm{C} ;{ }^{1} \mathrm{H}$ NMR $\left(600 \mathrm{MHz}, \mathrm{CDCl}_{3}, 25{ }^{\circ} \mathrm{C}\right): \delta=1.63-1.94(\mathrm{~m}$, $6 \mathrm{H}), 3.63-3.70(\mathrm{~m}, 1 \mathrm{H}), 3.94-4.02(\mathrm{~m}, 1 \mathrm{H}), 5.37-5.40(\mathrm{~m}, 1 \mathrm{H}), 6.02(\mathrm{~s}, 2 \mathrm{H}), 6.71(\mathrm{~s}, 1 \mathrm{H}), 7.45(\mathrm{~s}$, $1 \mathrm{H}), 8.52(\mathrm{~s}, 1 \mathrm{H}) ;{ }^{13} \mathrm{C}\left\{{ }^{1} \mathrm{H}\right\} \mathrm{NMR}\left(75.5 \mathrm{MHz}, \mathrm{CDCl}_{3}, 25{ }^{\circ} \mathrm{C}\right): \delta=19.6,25.2,28.8,62.9$, 101.1,102.1, 108.8, 112.6, 116.0, 124.8, 147.6, 149.5, 150.0; HRMS (EI-DFS) $m / z:[\mathrm{M}+\mathrm{H}]^{+} \mathrm{Calcd}$ for $\mathrm{C}_{13} \mathrm{H}_{14} \mathrm{BrNO}_{4}$ 327.0106; Found 327.0104.

(E)-2-Bromo-4,5-dimethoxybenzalehyde $O$-tetrahydro-2H-pyran-2-yl oxime (7h). (140 mg, 79\%, $E)$, white solid. $\mathrm{Mp}:=150-152{ }^{\circ} \mathrm{C} ;{ }^{1} \mathrm{H} \mathrm{NMR}\left(300 \mathrm{MHz}, \mathrm{CDCl}_{3}, 25^{\circ} \mathrm{C}\right): \delta=1.60-1.93(\mathrm{~m}, 6 \mathrm{H})$ 3.64-3.71 (m, 1H), $3.91(\mathrm{~s}, 3 \mathrm{H}) 3.93(\mathrm{~s}, 3 \mathrm{H}) 3.96-4.03(\mathrm{~m}, 1 \mathrm{H}) 5.41-5.44(\mathrm{~m}, 1 \mathrm{H}) 7.01(\mathrm{~s}, 1 \mathrm{H})$, $7.45(\mathrm{~s}, 1 \mathrm{H}), 8.52(\mathrm{~s}, 1 \mathrm{H}) ;{ }^{13} \mathrm{C}\left\{{ }^{1} \mathrm{H}\right\} \mathrm{NMR}\left(75.5 \mathrm{MHz}, \mathrm{CDCl}_{3}, 25{ }^{\circ} \mathrm{C}\right) \delta=19.6,25.2,28.9,56.1$, 
62.8, 101.0, 109.2, 115.1, 115.4, 123.5 148.5, 149.6, 151.2; HRMS (EI-DFS) $m / z:[\mathrm{M}+\mathrm{H}]^{+}$Calcd for $\mathrm{C}_{14} \mathrm{H}_{18} \mathrm{BrNO}_{4} 343.0419$; Found 343.0415.

(E)-[1,1'-Biphenyl]-4-carbaldehyde $O$-tetrahydro-2H-pyran-2-yl oxime (7i). (70 mg, 96\%, 1:1 $Z / E$ ), yellow solid. Mp: $76-78{ }^{\circ} \mathrm{C} ;{ }^{1} \mathrm{H}$ NMR $\left(300 \mathrm{MHz}, \mathrm{CDCl}_{3}, 25^{\circ} \mathrm{C}\right): \delta=1.60-1.96(\mathrm{~m}, 6 \mathrm{H})$, 3.67-3.73 (m, 1H), 3.97-4.05 (m, 1H), 5.43-5.45 (m, 1H), 7.36-7.42 (m, 1H), 7.45-7.50 (m, 2H), 7.62-7.65 (d, $J=8.1 \mathrm{~Hz} ; 4 \mathrm{H}), 7.72-7.75(\mathrm{~d}, J=8.3 \mathrm{~Hz} ; 2 \mathrm{H}), 8.27(\mathrm{~s}, 1 \mathrm{H}) ;{ }^{13} \mathrm{C}\left\{{ }^{1} \mathrm{H}\right\} \mathrm{NMR}(75.5$ $\left.\mathrm{MHz}, \mathrm{CDCl}_{3}, 25^{\circ} \mathrm{C}\right): \delta=19.8,25.2,28.9,63.0,101.1,127.1,127.3,127.7-127.9(\mathrm{~d}, J=9.6 \mathrm{~Hz})$, 128.9, 130.9, 140.4, 142.8, 150.1; HRMS (EI-DFS) $m / z$ : [M + H] ${ }^{+}$Calcd for $\mathrm{C}_{18} \mathrm{H}_{19} \mathrm{NO}_{2} 281.1416$; Found 281.1405.

Benzophenone $O$-tetrahydro- $2 H$-pyran-2-yl oxime (7j). (108 mg, 74\%), white solid. Mp: 64-66 ${ }^{\circ} \mathrm{C} ;{ }^{1} \mathrm{H}$ NMR $\left(300 \mathrm{MHz}, \mathrm{CDCl}_{3}, 25^{\circ} \mathrm{C}\right): \delta=1.53-1.81(\mathrm{~m}, 6 \mathrm{H}), 3.66-3.72(\mathrm{~m}, 1 \mathrm{H}), 3.86-3.93(\mathrm{~m}$, $1 \mathrm{H}), 5.48-5.51(\mathrm{t}, J=3.7 \mathrm{~Hz} ; 1 \mathrm{H}), 7.34-7.39(\mathrm{~m}, 3 \mathrm{H}), 7.45-7.46$ (m, 5H), 7.55-7.58 (dd, $J=6.1$, $1.9 \mathrm{~Hz} ; 2 \mathrm{H}) ;{ }^{13} \mathrm{C}\left\{{ }^{1} \mathrm{H}\right\} \mathrm{NMR}\left(75.5 \mathrm{MHz}, \mathrm{CDCl}_{3}, 25^{\circ} \mathrm{C}\right): \delta=19.5,25.3,28.8,62.7,101.1,127.9$, $128.1-128.3$ (d, $J=9.8 \mathrm{~Hz}), 128.8,129.3-129.4$ (d, $J=7.6 \mathrm{~Hz}), 130.1,132.4,133.5$, 136.4; HRMS (EI-DFS) $m / z:[\mathrm{M}+\mathrm{H}]^{+}$Calcd for $\mathrm{C}_{18} \mathrm{H}_{19} \mathrm{NO}_{2}$ 281.1416; Found 281.1410.

(E)-1,2-Diphenylethanone $O$-tetrahydro-2H-pyran-2-yl oxime (7k). (98 mg, 64\%, 1:>19 Z/E), clear oil. ${ }^{1} \mathrm{H}$ NMR $\left(300 \mathrm{MHz}, \mathrm{CDCl}_{3}, 25^{\circ} \mathrm{C}\right): \delta=1.48-1.91(\mathrm{~m}, 6 \mathrm{H}), 3.61-3.68(\mathrm{~m}, 1 \mathrm{H}), 3.77-$ $3.85(\mathrm{~m}, 1 \mathrm{H}), 4.15-4.20(\mathrm{~d}, J=14.5 \mathrm{~Hz} ; 1 \mathrm{H}), 4.32-4.36(\mathrm{~d}, J=14.5 \mathrm{~Hz} ; 1 \mathrm{H}), 5.52-5.55(\mathrm{t}, J=$ $3.0 \mathrm{~Hz} ; 1 \mathrm{H}), 7.20-7.24(\mathrm{~m}, 1 \mathrm{H}), 7.29-7.31(\mathrm{~d}, J=4.4 \mathrm{~Hz} ; 4 \mathrm{H}), 7.35-7.38(\mathrm{~m}, 3 \mathrm{H}), 7.73-7.76(\mathrm{~m}$, $2 \mathrm{H}) ;{ }^{13} \mathrm{C}\left\{{ }^{1} \mathrm{H}\right\} \mathrm{NMR}\left(75.5 \mathrm{MHz}, \mathrm{CDCl}_{3}, 25{ }^{\circ} \mathrm{C}\right) \delta=19.5,25.3,29.0,33.2,62.6,100.9,126.3$, 126.9, 128.4, 128.6 (d, $J=1.4 \mathrm{~Hz}), 129.3,135.6,137.0,157.6$; HRMS (EI-DFS) $m / z:[\mathrm{M}+\mathrm{H}]^{+}$ Calcd for $\mathrm{C}_{19} \mathrm{H}_{21} \mathrm{NO}_{2} 295.1572$; Found 295.1565.

(Z/E)-Furan-2-carbaldehyde $O$-tetrahydro- $2 H$-pyran-2-yl oxime (7l). (81 mg, 80\%, 1:6 Z/E), brown oil. ${ }^{1} \mathrm{H}$ NMR $\left(300 \mathrm{MHz}, \mathrm{CDCl}_{3}, 25^{\circ} \mathrm{C}\right): \delta=1.56-1.95(\mathrm{~m}, 6 \mathrm{H}, E$ and $Z), 3.64-3.71(\mathrm{~m}, 1 \mathrm{H}$, $E$ and $Z), 3.87-3.95(\mathrm{~m}, 1 \mathrm{H}, E$ and $Z), 5.36-5.39(\mathrm{~m}, 1 \mathrm{H}, Z), 5.44-5.46(\mathrm{~m}, 1 \mathrm{H}, E), 6.45-6.47(\mathrm{~m}$, $1 \mathrm{H}, Z), 6.54-6.56(\mathrm{~m}, 1 \mathrm{H}, E), 6.67-6.68(\mathrm{~d}, J=3.4 \mathrm{~Hz} ; 1 \mathrm{H}, Z), 7.27-7.29$ (d, $J=3.4 \mathrm{~Hz} ; 1 \mathrm{H}, E)$, 7.48-7.49 (d, $J=1.2 \mathrm{~Hz} ; 1 \mathrm{H}, E$ and $Z), 7.56(\mathrm{~s}, 1 \mathrm{H}, E), 8.08(\mathrm{~s}, 1 \mathrm{H}, Z) ;{ }^{13} \mathrm{C}\left\{{ }^{1} \mathrm{H}\right\} \mathrm{NMR}(75.5 \mathrm{MHz}$, $\mathrm{CDCl}_{\left.3,25^{\circ} \mathrm{C}\right)} \delta=19.7,19.8,25.1,28.6,28.8,62.5,63.1,101.2,111.6,112.2,113.1,117.9,137.5$, 140.5, 143.5, 144.3, 145.2; HRMS (EI-DFS) $m / z$ : $[\mathrm{M}+\mathrm{H}]^{+}$Calcd for $\mathrm{C}_{10} \mathrm{H}_{13} \mathrm{NO}_{3}$ 195.0895; Found 195.0892 .

(Z)-6-(Benzyloxy)- $1 \mathrm{H}$-indole-3-carbaldehyde $O$-tetrahydro- $2 \mathrm{H}$-pyran-2-yl oxime (7m'). (87 mg, $73 \%, 2: 1 \mathrm{Z} / \mathrm{E}$ ), white solid. Mp: $180-182{ }^{\circ} \mathrm{C}$; flash chromatography (hexanes:EtOAc/DCM = 80:16:4) followed by recrystallization (hexanes/chloroform); ${ }^{1} \mathrm{H}$ NMR $\left(300 \mathrm{MHz}, \mathrm{CDCl}_{3}, 25^{\circ} \mathrm{C}\right)$ : $\delta=1.60-1.94(\mathrm{~m}, 6 \mathrm{H}), 3.66-3.72(\mathrm{~m}, 1 \mathrm{H}), 4.00-4.01(\mathrm{~m}, 1 \mathrm{H}) 5.15(\mathrm{~s}, 2 \mathrm{H}), 5.44-5.46(\mathrm{~m}, 1 \mathrm{H})$, 6.97-7.01 (d $J=2.4,8.8 \mathrm{~Hz} ; 1 \mathrm{H}), 7.26-7.29(\mathrm{~m}, 1 \mathrm{H}), 7.34-7.43(\mathrm{~m}, 4 \mathrm{H}), 7.50-7.53$ (d $J=7.1 \mathrm{~Hz}$; 2H) 7.77-7.78 (d, $J=2.3 \mathrm{~Hz} ; 1 \mathrm{H}), 8.32(\mathrm{~s}, 1 \mathrm{H}), 8.41(\mathrm{~s}, 1 \mathrm{H}) ;{ }^{13} \mathrm{C}\left\{{ }^{1} \mathrm{H}\right\}$ NMR $\left(75.5 \mathrm{MHz}, d_{6^{-}}\right.$ DMSO, $\left.25^{\circ} \mathrm{C}\right) \delta=20.0,25.4,29.3,62.2,70.2,100.5,105.8108 .5,112.6,113.1,125.1,128.1(\mathrm{~d}$, $J=5.4 \mathrm{~Hz}$ ), 130.7, 132.4, 138.1, 146.9, 153.7; HRMS (EI-DFS) $m / z$ : $[\mathrm{M}+\mathrm{H}]^{+}$Calcd for $\mathrm{C}_{21} \mathrm{H}_{22} \mathrm{~N}_{2} \mathrm{O}_{3} 350.1630$; Found 350.1625.

(1(Z/E),2E)-Cinnamaldehyde $O$-tetrahydro-2H-pyran-2-yl oxime (7n). (66 mg, 55\%, 1:1.2 Z/E), clear yellow oil. ${ }^{1} \mathrm{H}$ NMR (300 MHz, $\left.\mathrm{CDCl}_{3}, 25^{\circ} \mathrm{C}\right): \delta=1.56-1.92(\mathrm{~m}, 6 \mathrm{H}, E$ and $Z$ ), 3.63-3.70 
(m, 1H, $E$ and $Z$ ), 3.93-4.01 (m, 1H, $E$ and $Z), 4.96-4.99(\mathrm{~m}, 1 \mathrm{H}, Z) 5.30-5.32(\mathrm{~m}, 1 \mathrm{H}, E), 6.81-$ 6.99 (m, 1H, $E$ and $Z$ ), 7.31-7.40 (overlapping signals, $E$ and $Z$ ), 7.44-7.47 (dd, $J=6.7,1.1 \mathrm{~Hz}$; $2 \mathrm{H}, E$ and $Z), 7.99-8.03(\mathrm{~d}, J=9.2 \mathrm{~Hz} ; 1 \mathrm{H}, E) ;{ }^{13} \mathrm{C}\left\{{ }^{1} \mathrm{H}\right\}$ NMR $\left(75.5 \mathrm{MHz}, \mathrm{CDCl}_{3}, 25{ }^{\circ} \mathrm{C}\right): \delta=$ 19.8, 25.1, 25.5, 28.8, 30.7, 63.1, 94.7, 100.9, 122.1, 127.0, 128.8 (d, $J=6.8 \mathrm{~Hz}), 135.9,139.2$, 152.2; HRMS (EI-DFS) $m / z$ : $[\mathrm{M}+\mathrm{H}]^{+}$Calcd for $\mathrm{C}_{14} \mathrm{H}_{17} \mathrm{NO}_{2} 231.1259$; Found 231.1256.

(E)-3-(4-(tert-Butyl)-phenyl)-2-methylpropanal $O$-tetrahydro-2H-pyran-2-yl oxime (7o). (80 mg, $77 \%, 1: 2 \mathrm{Z} / E)$, clear oil. ${ }^{1} \mathrm{H}$ NMR $\left(300 \mathrm{MHz}, \mathrm{CDCl}_{3}, 25^{\circ} \mathrm{C}\right): \delta=1.08-1.11(\mathrm{~d}, J=6.7 \mathrm{~Hz} ; 3 \mathrm{H})$, $1.34(\mathrm{~s}, 9 \mathrm{H}), 1.59-1.85(\mathrm{~m}, 6 \mathrm{H}), 2.55-2.64(\mathrm{~m}, 1 \mathrm{H}), 2.70-2.91(\mathrm{~m}, 2 \mathrm{H}), 3.57-3.67(\mathrm{~m}, 1 \mathrm{H}), 3.85-$ $3.99(\mathrm{~m}, 1 \mathrm{H}), 5.21-5.25(\mathrm{~m}, 1 \mathrm{H}), 7.11-7.14(\mathrm{~d}, J=8.0 \mathrm{~Hz} ; 2 \mathrm{H}), 7.31-7.34(\mathrm{~m}, 2 \mathrm{H}), 7.44-7.47$ $(\mathrm{dd}, J=5.4,1.5 \mathrm{~Hz} ; 1 \mathrm{H}) ;{ }^{13} \mathrm{C}\left\{{ }^{1} \mathrm{H}\right\} \mathrm{NMR}\left(75.5 \mathrm{MHz}, \mathrm{CDCl}_{3}, 25{ }^{\circ} \mathrm{C}\right) \delta=17.4(\mathrm{~d}, J=7.6 \mathrm{~Hz}), 19.9$, 20.1, 25.2, 28.9 (d, $J=5.5 \mathrm{~Hz}), 31.4,34.4,36.0$ (d, $J=2.8 \mathrm{~Hz}), 40.3,40.4,62.9,63.2,100.3,100.6$, 125.2, $128.9136 .0\left(\mathrm{~d}, J=2.4 \mathrm{~Hz}\right.$ ), 149.0, 156.6; HRMS (EI-DFS) $m / z$ : $[\mathrm{M}+\mathrm{H}]^{+} \mathrm{Calcd}$ for $\mathrm{C}_{19} \mathrm{H}_{29} \mathrm{NO}_{2}$ 303.2198; Found 303.2191.

Cyclopentanone $O$-tetrahydro- $2 H$-pyran-2-yl oxime (7p). (85 mg, 89\%), clear oil. ${ }^{1} \mathrm{H}$ NMR (300 $\left.\mathrm{MHz}, \mathrm{CDCl}_{3}, 25{ }^{\circ} \mathrm{C}\right): \delta=1.54-1.85(\mathrm{~m}, 10 \mathrm{H}), 2.36-2.50(\mathrm{~m}, 4 \mathrm{H}), 3.56-3.64(\mathrm{~m}, 1 \mathrm{H}), 3.90-3.97$ $(\mathrm{m}, 1 \mathrm{H}), 5.16-5.19(\mathrm{~m}, 1 \mathrm{H},) ;{ }^{13} \mathrm{C}\left\{{ }^{1} \mathrm{H}\right\} \operatorname{NMR}\left(75.5 \mathrm{MHz}, \mathrm{CDCl}_{3}, 25^{\circ} \mathrm{C}\right) \delta=20.3,24.6,25.1,25.2$, 27.8, 29.1, 31.1, 63.5, 100.7, 168.3; HRMS (EI-DFS) $m / z$ : $[\mathrm{M}+\mathrm{H}]^{+}$Calcd for $\mathrm{C}_{10} \mathrm{H}_{17} \mathrm{NO}_{2}$ 183.1259; Found 183.1252.

Cycloheptanone $O$-tetrahydro- $2 \mathrm{H}$-pyran-2-yl oxime (7q). (79 mg, 72\%), clear oil. ${ }^{1} \mathrm{H}$ NMR (300 $\left.\mathrm{MHz}, \mathrm{CDCl}_{3}, 25{ }^{\circ} \mathrm{C}\right): \delta=1.52-1.84(\mathrm{~m}, 14 \mathrm{H}), 2.40-2.44(\mathrm{~m}, 2 \mathrm{H}), 2.59-2.63(\mathrm{~m}, 2 \mathrm{H}), 3.58-3.65$ $(\mathrm{m}, 1 \mathrm{H}), 3.88-3.95(\mathrm{~m} \mathrm{1H}), 5.18-5.20(\mathrm{~m}, 1 \mathrm{H}) ;{ }^{13} \mathrm{C}\left\{{ }^{1} \mathrm{H}\right\} \mathrm{NMR}\left(75.5 \mathrm{MHz}, \mathrm{CDCl}_{3}, 25^{\circ} \mathrm{C}\right) \delta=20.3$, 24.6, 25.3, 27.6, 29.2 (d, $J=6.5 \mathrm{~Hz}), 30.4$ (d, $J=4.6 \mathrm{~Hz}), 33.8$, 63.3, 100.5, 165.5; HRMS (EIDFS) $m / z:[\mathrm{M}+\mathrm{H}]^{+}$Calcd for $\mathrm{C}_{12} \mathrm{H}_{21} \mathrm{NO}_{2} 211.1572$; Found 211.1565.

(Z/E)-1,6,6-Trimethylbicyclo[3.1.1]heptan-3-one $O$-tetrahydro-2H-pyran-2-yl oxime (7r). (97 mg, 74\%, 1:1 Z/E), clear oil. ${ }^{1} \mathrm{H}$ NMR $\left(300 \mathrm{MHz}, \mathrm{CDCl}_{3}, 25^{\circ} \mathrm{C}\right): \delta=0.802(\mathrm{~s}, 3 \mathrm{H}, E$ and $Z), 0.907$ (s, 3H, $E$ and $Z$ ), 1.04, (s, 3H, $E$ and $Z$ ), 1.18-1.29 (m, 1H, $E$ and $Z$ ), 1.49-1.90 (overlapping signals, $10 \mathrm{H}, E$ and $Z), 2.04(\mathrm{~s}, 1 \mathrm{H}, Z), 2.11(\mathrm{~s}, 1 \mathrm{H}, E), 2.51-2.60(\mathrm{~m}, 1 \mathrm{H}, E$ and $Z), 3.56-3.63(\mathrm{~m}$, $1 \mathrm{H}, E$ and $Z)$, 3.82-3.97 (m, $1 \mathrm{H}, E$ and $Z), 5.17-5.20(\mathrm{~m}, 1 \mathrm{H}, E), 5.23-5.26(\mathrm{~m}, 1 \mathrm{H}, Z) ;{ }^{13} \mathrm{C}\left\{{ }^{1} \mathrm{H}\right\}$ NMR $\left(75.5 \mathrm{MHz}, \mathrm{CDCl}_{3}, 2{ }^{\circ} \mathrm{C}\right) \delta=11.1,11.2,18.5,18.5,19.5,19.6,19.9,20.4,25.3,25.4,27.2$, 27.3, 29.3, 29.4, 32.7, 32.8, 33.8, 33.9, 43.6, 43.7, 48.0, 48.3, 51.8, 51.9, 62.6, 63.5, 100.0, 100.8, 170.8, 171.0; HRMS (EI-DFS) $m / z$ : $[\mathrm{M}+\mathrm{H}]^{+}$Calcd for $\mathrm{C}_{15} \mathrm{H}_{25} \mathrm{NO}_{2} 251.1885$; Found 251.1883 .

(E)-4-(4-Methylpent-3-en-1-yl)cyclohex-3-enecarbaldehyde $O$-tetrahydro-2H-pyran-2-yl oxime (7s). (94 mg, 95\%, 1:2 Z/E), clear oil. ${ }^{1} \mathrm{H} \mathrm{NMR}\left(300 \mathrm{MHz}, \mathrm{CDCl}_{3}, 25^{\circ} \mathrm{C}\right): \delta=1.60$ (overlapping signals, $5 \mathrm{H}) 1.69(\mathrm{~s}, 3 \mathrm{H}), 1.80-2.23$ (overlapping signals, $14 \mathrm{H}), 2.50-2.60(\mathrm{~m}, 1 \mathrm{H}), 3.59-3.65(\mathrm{~m}$, $1 \mathrm{H}), 3.91-3.97(\mathrm{~m}, 1 \mathrm{H}), 5.06-5.11(\mathrm{~m}, 1 \mathrm{H}), 5.19-5.21(\mathrm{~m}, 1 \mathrm{H}), 5.38-5.43(\mathrm{~m}, 1 \mathrm{H}), 7.43-7.47$ (dd, $J=7.3,3.5 \mathrm{~Hz} ; 1 \mathrm{H}) ;{ }^{13} \mathrm{C}\left\{{ }^{1} \mathrm{H}\right\} \operatorname{NMR}\left(75.5 \mathrm{MHz}, \mathrm{CDCl}_{3}, 25^{\circ} \mathrm{C}\right): \delta=17.7,20.1,25.2,25.7,26.3$, $26.8(\mathrm{~d}, J=5.6 \mathrm{~Hz}), 27.1(\mathrm{~d}, J=3.3 \mathrm{~Hz}), 28.9,34.4(\mathrm{~d}, J=4.3 \mathrm{~Hz}), 34.9,37.6,63.3,100.6,118.5$, 120.5 (d, $J=3.4 \mathrm{~Hz}), 124.2,131.4,135.8,137.6,156.3$ (d, $J=3.1 \mathrm{~Hz})$; HRMS (EI-DFS) $m / z$ : [M $+\mathrm{H}]^{+}$Calcd for $\mathrm{C}_{18} \mathrm{H}_{29} \mathrm{NO}_{2}$ 291.2198; Found 291.2190.

(Z)-4-(4-Methylpent-3-en-1-yl)cyclohex-3-enecarbaldehyde $O$-tetrahydro-2H-pyran-2-yl oxime (7s'). (45 mg, 86\%,), clear oil. ${ }^{1} \mathrm{H} \mathrm{NMR}\left(300 \mathrm{MHz}, \mathrm{CDCl}_{3}, 25^{\circ} \mathrm{C}\right): \delta=1.62$ (overlapping signals, 
$5 \mathrm{H}), 1.70(\mathrm{~s}, 3 \mathrm{H}), 1.77-2.23$ (overlapping signals, $14 \mathrm{H}), 3.14-3.24(\mathrm{~m}, 1 \mathrm{H}), 3.63-3.68(\mathrm{~m}, 1 \mathrm{H})$, 3.88-3.97 (m, 1H), 5.09-5.13 (m, 1H), 5.24-5.28 (m, 1H), 5.41-5.45 (m, 1H), 6.68-6.71 (d, $J=$ $7.1 \mathrm{~Hz} ; 1 \mathrm{H}) ;{ }^{13} \mathrm{C}\left\{{ }^{1} \mathrm{H}\right\} \mathrm{NMR}\left(75.5 \mathrm{MHz}, \mathrm{CDCl}_{3}, 25^{\circ} \mathrm{C}\right): \delta=17.7,19.9(\mathrm{~d}, J=6.4 \mathrm{~Hz}), 25.2,25.7$, 26.4, $27.0(\mathrm{~d}, J=2.2 \mathrm{~Hz}), 28.4,28.8,30.7$ (d, $J=2.1 \mathrm{~Hz}), 37.7,62.9,63.2,100.7,118.8,120.6$, $124.2,131.5,137.7,156.9(\mathrm{~d}, J=4.8 \mathrm{~Hz})$.

(Z/E)-3-Methyl-2-((Z)-pent-2-en-1-yl)cyclopent-2-enone $O$-tetrahydro-2H-pyran-2-yl oxime (7t). (59 mg, 43\%, C.N.D.) clear oil. ${ }^{1} \mathrm{H}$ NMR $\left(300 \mathrm{MHz}, \mathrm{CDCl}_{3}, 25{ }^{\circ} \mathrm{C}\right): \delta=0.963-1.01(\mathrm{t}, J=7.5$ $\mathrm{Hz}$; 3H), 1.57-1.86 (m, 6H), $1.87(\mathrm{~s}, 3 \mathrm{H}), 2.15-2.19(\mathrm{~m}, 2 \mathrm{H}), 2.37-2.41(\mathrm{~m}, 2 \mathrm{H}), 2.65-2.69(\mathrm{~m}$, 2H), 2.90-3.10 (qd, $J=14.7,4.6 \mathrm{~Hz} ; 2 \mathrm{H}), 3.57-3.64(\mathrm{~m}, 1 \mathrm{H}), 3.91-3.98(\mathrm{~m}, 1 \mathrm{H}), 5.24-5.26$ (m, 1H), 5.35-5.38 (t, $J=4.8 \mathrm{~Hz}, 2 \mathrm{H}) ;{ }^{13} \mathrm{C}\left\{{ }^{1} \mathrm{H}\right\} \mathrm{NMR}\left(75.5 \mathrm{MHz}, \mathrm{CDCl}_{3}, 25{ }^{\circ} \mathrm{C}\right) \delta=14.215 .4,20.2$, 20.5, 22.5, 25.1, 25.4, 29.4, 34.3, 63.2, 101.0, 125.9, 133.3, 151.4, 168.7; HRMS (EI-DFS) $m / z$ : $[\mathrm{M}+\mathrm{H}]^{+}$Calcd for $\mathrm{C}_{16} \mathrm{H}_{25} \mathrm{NO}_{2} 263.1885$; Found 263.1881. Note: the $Z / E$ isomer configuration could not be determined (C.N.D.) for this compound.

(Z/E)-tert-Buty-1,4-((((tetrahydro-2H-pyran-2-yl)oxy)imino)methyl)piperidine-1-carboxylate (7u). (87 mg, 54\% 1:2 Z/E), clear oil. ${ }^{1} \mathrm{H} \mathrm{NMR}\left(300 \mathrm{MHz}, \mathrm{CDCl}_{3}, 25{ }^{\circ} \mathrm{C}\right): \delta=1.44(\mathrm{~s}, 9 \mathrm{H}, E$ and $Z), 1.51-1.84$ (overlapping signals, $10 \mathrm{H}, E$ and $Z), 2.39-2.50(\mathrm{~m}, 1 \mathrm{H}, E), 2.73-2.81(\mathrm{~m}, 2 \mathrm{H}, E$ and $Z), 3.02-3.10(\mathrm{~m}, 1 \mathrm{H}, Z), 3.56-3.63(\mathrm{~m}, 1 \mathrm{H}, E$ and $Z), 3.88-3.95(\mathrm{~m}, 1 \mathrm{H}, E$ and $Z), 4.05-4.09(\mathrm{~m}$, $2 \mathrm{H}, E$ and $Z), 5.17-5.19(\mathrm{~m}, 1 \mathrm{H}, E), 5.21-5.22(\mathrm{~m}, 1 \mathrm{H}, Z) 6.59-6.61(\mathrm{~d}, J=6.9 \mathrm{~Hz} ; 1 \mathrm{H}, Z), 7.37-$ $7.39(\mathrm{~d}, J=6.5 \mathrm{~Hz} ; 1 \mathrm{H}, E) ;{ }^{13} \mathrm{C}\left\{{ }^{1} \mathrm{H}\right\} \operatorname{NMR}\left(75.5 \mathrm{MHz}, \mathrm{CDCl}_{3}, 25{ }^{\circ} \mathrm{C}\right) \delta=20.0,25.1,28.4,28.9$, 29.2, 29.3, 36.9, 63.3, 79.5, 100.7, 154.8, 155.2; HRMS (EI-DFS) $m / z:[\mathrm{M}+\mathrm{H}]^{+}$Calcd for $\mathrm{C}_{16} \mathrm{H}_{29} \mathrm{~N}_{2} \mathrm{O}_{4}$ 313.2127; Found 313.2119.

(Z/E)-2,2,7,7-Tetramethyltetrahydro-3aH-bis([1,3]dioxolo)[4,5-b4',5'-d]pyran-5-carbaldehyde $O$-tetrahydro-2 $H$-pyran-2-yl oxime (7v). (97 mg, 79\%, 1:2, Z/E), yellow oil. ${ }^{1} \mathrm{H}$ NMR (300 MHz, $\left.\mathrm{CDCl}_{3}, 25^{\circ} \mathrm{C}\right): \delta=1.33-1.36(\mathrm{t}, J=4.4 \mathrm{~Hz} ; 6 \mathrm{H}, E$ and $Z), 1.49(\mathrm{~s}, 3 \mathrm{H}, E$ and $Z), 1.56(\mathrm{~s}, 3 \mathrm{H}, E$ and $Z), 1.59-1.83(\mathrm{~m}, 6 \mathrm{H}, E$ and $Z), 3.60-3.70(\mathrm{~m}, 1 \mathrm{H}, E$ and $Z), 3.79-3.95(\mathrm{~m}, 1 \mathrm{H}, E$ and $Z$ ), 4.29-4.40 (m, 1H, $E$ and $Z$ ), 4.50-4.68 (overlapping signals, $2 \mathrm{H}, E$ and $Z$ ), 5.00-5.05 (td, $J=5.2$, $1.9 \mathrm{~Hz} ; 1 \mathrm{H}, E), 5.27-5.31(\mathrm{~m}, 1 \mathrm{H}, E) 5.38-5.41(\mathrm{t}, J=2.9 \mathrm{~Hz} ; 1 \mathrm{H}, Z), 5.55-5.59(\mathrm{~m}, 1 \mathrm{H}, E$ and $Z), 6.87-6.91(\mathrm{~m}, 1 \mathrm{H}, E), 7.51-7.56(\mathrm{~m}, 1 \mathrm{H}, Z) ;{ }^{13} \mathrm{C}\left\{{ }^{1} \mathrm{H}\right\} \mathrm{NMR}\left(75.5 \mathrm{MHz}, \mathrm{CDCl}_{3}, 25{ }^{\circ} \mathrm{C}\right) \delta=$ 19.0, 19.3, 19.5, 19.7, 19.9, 24.3 (d, $J=5.0 \mathrm{~Hz}), 24.5,24.8,25.0$ (d, $J=2.0 \mathrm{~Hz}$ ), 25.1, 25.9, 26.0 $(\mathrm{d}, J=3.9 \mathrm{~Hz}), 26.1,28.4,28.6(\mathrm{~d}, J=4.7 \mathrm{~Hz}), 62.1,62.3,62.9,63.5,63.9,66.5(\mathrm{~d}, J=3.2 \mathrm{~Hz})$, $70.1,70.2,70.3,70.6(\mathrm{~d}, J=4.4 \mathrm{~Hz}), 71.4,71.5,72.9,73.6,96.1,96.2(\mathrm{~d}, J=2.8 \mathrm{~Hz}), 100.2,100.3$, 100.7, 101.0, 108.9, 109.6, 109.7; HRMS (EI-DFS) $m / z$ : $[\mathrm{M}+\mathrm{H}]^{+}$Calcd for $\mathrm{C}_{17} \mathrm{H}_{27} \mathrm{NO}_{7} 357.1788$; Found 357.1780.

(E)-3,7-Dimethloct-6-enal $O$-tetrahydro-2H-pyran-2-yl oxime (7w). (28 mg, 39\%, 1:1.2 Z/E), clear oil. ${ }^{1} \mathrm{H}$ NMR $\left(300 \mathrm{MHz}, \mathrm{CDCl}_{3}, 25{ }^{\circ} \mathrm{C}\right): \delta=0.923-0.952(\mathrm{dd}, J=4.6,2.1 \mathrm{~Hz} ; 3 \mathrm{H}), 1.17-$ $1.44(\mathrm{~m}, 4 \mathrm{H}), 1.59(\mathrm{~s}, 3 \mathrm{H}), 1.67(\mathrm{~s}, 3 \mathrm{H}), 1.76-2.29$ (overlapping signals, 9H), 3.56-3.64 (m, 1H), 3.89-3.96 (m, 1H), 5.05-5.10 (m, 1H), 5.19-5.22 (m, 1H), 7.47-7.52 (t, J=6.5 Hz; $1 \mathrm{H}) ;{ }^{13} \mathrm{C}\left\{{ }^{1} \mathrm{H}\right\}$ $\operatorname{NMR}\left(75.5 \mathrm{MHz}, \mathrm{CDCl}_{3}, 25^{\circ} \mathrm{C}\right): \delta=17.6,19.4(\mathrm{~d}, J=6.6 \mathrm{~Hz}), 20.0(\mathrm{~d}, J=1.7 \mathrm{~Hz}), 25.2,25.4$ $(\mathrm{d}, J=2.4 \mathrm{~Hz}), 25.7,28.9,31.0(\mathrm{~d}, J=6.6 \mathrm{~Hz}), 36.4(\mathrm{~d}, J=2.1 \mathrm{~Hz}), 36.6,36.8,63.1(\mathrm{~d}, J=4.2$ $\mathrm{Hz}), 100.5(\mathrm{~d}, J=3.5 \mathrm{~Hz}), 124.3,131.5,152.2(\mathrm{~d}, J=2.1 \mathrm{~Hz})$; HRMS (EI-DFS) $m / z:[\mathrm{M}+\mathrm{H}]^{+}$ Calcd for $\mathrm{C}_{15} \mathrm{H}_{28} \mathrm{NO}_{2}$ 254.2120; Found 254.2000. 
(E)-Ethyl-2-cyano-2-(((tetrahydro-2H-pyran-2-yl)oxy)imino)acetate. (7x). (98 mg, 83\%, E), clear oil. ${ }^{1} \mathrm{H}$ NMR $\left(300 \mathrm{MHz}, \mathrm{CDCl}_{3}, 25{ }^{\circ} \mathrm{C}\right): \delta=1.39-1.44$ (t, $\left.J=7.1 \mathrm{~Hz} ; 3 \mathrm{H}\right) 1.64-2.02(\mathrm{~m}, 6 \mathrm{H})$, $3.72-3.79(\mathrm{~m}, 1 \mathrm{H}), 3.82-3.90(\mathrm{td}, J=10.5,2.9 \mathrm{~Hz} ; 1 \mathrm{H}), 4.40-4.48(\mathrm{dq}, J=7.1,1.8 \mathrm{~Hz} ; 2 \mathrm{H}), 5.70-$ $5.71(\mathrm{~m}, 1 \mathrm{H}) ;{ }^{13} \mathrm{C}\left\{{ }^{1} \mathrm{H}\right\}$ NMR $\left(75.5 \mathrm{MHz}, \mathrm{CDCl}_{3}, 2{ }^{\circ} \mathrm{C}\right) \delta=14.0,18.1,24.6,27.9,62.6,63.6$, 104.7, 107.6, 157.8; HRMS (CI-DFS) $m / z$ : $[\mathrm{M}+\mathrm{H}]^{+}$Calcd for $\mathrm{C}_{10} \mathrm{H}_{15} \mathrm{~N}_{2} \mathrm{O}_{4}$ 227.1032; Found 227.1027.

(E)-Acetophenone $O$-(1-ethoxyethyl) oxime (7y). (99 mg, 92\%, 1:19 Z/E), clear oil. ${ }^{1} \mathrm{H}$ NMR (300 $\left.\mathrm{MHz}, \mathrm{CDCl}_{3}, 25^{\circ} \mathrm{C}\right): \delta=1.24-1.29(\mathrm{t}, J=7.1 \mathrm{~Hz}, 3 \mathrm{H}), 1.51-1.53(\mathrm{~d}, J=5.4 \mathrm{~Hz} ; 3 \mathrm{H}), 2.30(\mathrm{~s}$, $3 \mathrm{H}), 3.65-3.75(\mathrm{~m}, 1 \mathrm{H}), 3.90-4.00(\mathrm{~m}, 1 \mathrm{H}), 5.43-5.49(\mathrm{q}, J=5.4 \mathrm{~Hz} ; 1 \mathrm{H}), 7.38-7.41(\mathrm{~m}, 3 \mathrm{H})$, 7.68-7.71 (m, 2H); ${ }^{13} \mathrm{C}\left\{{ }^{1} \mathrm{H}\right\}$ NMR $\left(75.5 \mathrm{MHz}, \mathrm{CDCl}_{3}, 25^{\circ} \mathrm{C}\right): \delta=12.9,15.4,20.4,64.2,103.8$, 126.1, 128.4, 129.1, 136.7, 154.4; HRMS (EI-DFS) $m / z$ : $[\mathrm{M}+\mathrm{H}]^{+}$Calcd for $\mathrm{C}_{12} \mathrm{H}_{17} \mathrm{NO}_{2} 207.1259$; Found 207.1252.

(E)-Acetophenone $O$-(1-butoxyethyl) oxime (7z). (116 mg, 95\%, 1:19 Z/E), clear oil. ${ }^{1} \mathrm{H}$ NMR $\left(300 \mathrm{MHz}, \mathrm{CDCl}_{3}, 25^{\circ} \mathrm{C}\right): \delta=0.95-0.97(\mathrm{~m}, 3 \mathrm{H}), 1.36-1.46(\mathrm{~m}, 2 \mathrm{H}), 1.50-1.52(\mathrm{~d}, J=5.5 \mathrm{~Hz}$; $3 \mathrm{H}), 1.60-1.66(\mathrm{~m}, 2 \mathrm{H}), 2.30(\mathrm{~s}, 3 \mathrm{H}), 3.60-3.67(\mathrm{~m}, 1 \mathrm{H}), 3.86-3.93(\mathrm{~m}, 1 \mathrm{H}), 5.42-5.47$ (q, $J=5.4$ $\mathrm{Hz} ; 1 \mathrm{H}), 7.38-7.40(\mathrm{~m}, 3 \mathrm{H}), 7.68-7.71(\mathrm{~m}, 2 \mathrm{H}) ;{ }^{13} \mathrm{C}\left\{{ }^{1} \mathrm{H}\right\}$ NMR $\left(75.5 \mathrm{MHz}, \mathrm{CDCl}_{3}, 25^{\circ} \mathrm{C}\right): \delta=$ 12.8, 13.9, 19.3, 20.3, 32.0, 68.5, 104.0, 126.1, 128.3, 129.0, 136.8, 154.4; HRMS (EI-DFS) $m / z$ : $[\mathrm{M}+\mathrm{H}]^{+}$Calcd for $\mathrm{C}_{14} \mathrm{H}_{21} \mathrm{NO}_{2} 235.1572$; Found 235.1564.

(E)-Acetophenone $O$-(1-isobutoxyethyl) oxime (7aa). (109 mg, 89\%, 1:19 Z/E), clear oil. ${ }^{1} \mathrm{H}$ NMR $\left(300 \mathrm{MHz}, \mathrm{CDCl}_{3}, 25^{\circ} \mathrm{C}\right): \delta=0.929-0.964(\mathrm{q}, J=3.5 \mathrm{~Hz} ; 6 \mathrm{H}), 1.50-1.52(\mathrm{~d}, J=5.4 \mathrm{~Hz} ; 3 \mathrm{H})$, $1.83-1.97(\mathrm{~m}, 1 \mathrm{H}) 2.30(\mathrm{~s}, 3 \mathrm{H}), 3.37-3.42(\mathrm{~m}, 1 \mathrm{H}), 3.63-3.68(\mathrm{~m}, 1 \mathrm{H}), 5.40-5.45$ (q, $J=5.4 \mathrm{~Hz}$; $1 \mathrm{H}), 7.38-7.41(\mathrm{~m}, 3 \mathrm{H}), 7.68-7.71(\mathrm{~m}, 2 \mathrm{H}) ;{ }^{13} \mathrm{C}\left\{{ }^{1} \mathrm{H}\right\} \mathrm{NMR}\left(75.5 \mathrm{MHz}, \mathrm{CDCl}_{3}, 25^{\circ} \mathrm{C}\right): \delta=12.9$, $19.4(\mathrm{~d}, J=6.8 \mathrm{~Hz}), 20.2,28.7,75.6,104.1,126.1,128.4,129.0,136.8,154.4$; HRMS (EI-DFS) $m / z:[\mathrm{M}+\mathrm{H}]^{+}$Calcd for $\mathrm{C}_{14} \mathrm{H}_{21} \mathrm{NO}_{2} 235.1572$; Found 235.1566.

(E)-Acetophenone $O$-(2-ethoxypropan-2-yl) oxime (7ab). (75 mg, 65\%, 1:19 Z/E), clear oil. ${ }^{1} \mathrm{H}$ NMR (300 MHz, $\left.\mathrm{CDCl}_{3}, 25^{\circ} \mathrm{C}\right): \delta=1.19-1.23$ (t, $\left.J=7.1 \mathrm{~Hz} ; 3 \mathrm{H}\right), 1.61(\mathrm{~s}, 6 \mathrm{H}), 2.28(\mathrm{~s}, 3 \mathrm{H})$, $3.58-3.65(\mathrm{q}, J=7.1 \mathrm{~Hz} ; 2 \mathrm{H}), 7.38-7.40(\mathrm{~m}, 3 \mathrm{H}), 7.73-7.76(\mathrm{~m}, 2 \mathrm{H}) ;{ }^{13} \mathrm{C}\left\{{ }^{1} \mathrm{H}\right\} \mathrm{NMR}(75.5 \mathrm{MHz}$, $\left.\mathrm{CDCl}_{3}, 25{ }^{\circ} \mathrm{C}\right): \delta=12.5,15.7,24.7,57.0,103.7,126.2,128.3,128.9,137.1,154.1$; HRMS (EIDFS) $m / z:[\mathrm{M}+\mathrm{H}]^{+}$Calcd for $\mathrm{C}_{13} \mathrm{H}_{19} \mathrm{NO}_{2} 221.1416$; Found 221.1408.

(E)-Acetophenone $O$-(1-(cyclohexyloxy)ethyl) oxime (7ac). $(65 \mathrm{mg}, 48 \%, 1: 19 \mathrm{Z} / \mathrm{E})$, clear oil. ${ }^{1} \mathrm{H}$ NMR (300 MHz, $\left.\mathrm{CDCl}_{3}, 25^{\circ} \mathrm{C}\right): \delta=1.19-1.42(\mathrm{~m}, 5 \mathrm{H}), 1.49-1.50(\mathrm{~d}, J=5.4 \mathrm{~Hz} ; 3 \mathrm{H}), 1.53-1.58$ $(\mathrm{m}, 1 \mathrm{H}), 1.74-1.79(\mathrm{~m}, 2 \mathrm{H}), 1.91-2.00(\mathrm{~m}, 2 \mathrm{H}), 2.28(\mathrm{~s}, 3 \mathrm{H}), 3.78-3.87(\mathrm{~m}, 1 \mathrm{H}), 5.53-5.59(\mathrm{q}, J$ $=5.4 \mathrm{~Hz} ; 1 \mathrm{H}), 7.37-7.41(\mathrm{~m}, 3 \mathrm{H}), 7.67-7.70(\mathrm{~m}, 2 \mathrm{H}) ;{ }^{13} \mathrm{C}\{1 \mathrm{H}\}$ NMR $\left(75.5 \mathrm{MHz}, \mathrm{CDCl}_{3}, 25{ }^{\circ} \mathrm{C}\right)$ : $\delta=12.9,20.9,24.2,24.4,25.7,32.4,32.5,33.6,76.3,102.0,126.0,128.4,129.0,136.8,154.3$; HRMS (EI-DFS) $m / z$ : $[\mathrm{M}+\mathrm{H}]^{+}$Calcd for $\mathrm{C}_{16} \mathrm{H}_{23} \mathrm{NO}_{2}$ 261.1729; Found 261.1725.

(E)-Acetophenone $O$-tetrahydrofuran-2-yl oxime (7ad). (92 mg, 87\%, 1:19 Z/E), clear oil. ${ }^{1} \mathrm{H}$ NMR $\left(300 \mathrm{MHz}, \mathrm{CDCl}_{3}, 25{ }^{\circ} \mathrm{C}\right): \delta=1.89-2.17(\mathrm{~m}, 4 \mathrm{H}), 2.26(\mathrm{~s}, 3 \mathrm{H}), 3.96-4.09$ (overlapping signals, 2H), 5.93-5.95 (t, $J=3.5 \mathrm{~Hz} ; 1 \mathrm{H}), 7.36-7.38(\mathrm{~m}, 3 \mathrm{H}), 7.69-7.72(\mathrm{~m}, 2 \mathrm{H}) ;{ }^{13} \mathrm{C}\left\{{ }^{1} \mathrm{H}\right\} \mathrm{NMR}$ (75.5 MHz, $\left.\mathrm{CDCl}_{3}, 25^{\circ} \mathrm{C}\right): \delta=12.9,24.0,30.9,67.9,106.5,126.2,128.3,129.1,136.5,155.8$; HRMS (EI-DFS) $m / z$ : [M + H] ${ }^{+}$Calcd for $\mathrm{C}_{12} \mathrm{H}_{15} \mathrm{NO}_{2}$ 205.1103; Found 205.1099. 
(E)-Acetophenone $O$-(1-(( $(3 S, 8 S, 9 S, 10 R, 13 R, 14 S, 17 R)-10,13$-dimethyl-17-((R)-6-methylheptan2-yl)-2,3,4,7,8,9,10,11,12,13,14,15,16,17-tetradecahydro-1H-cyclopenta[a]phenanthren-3yl)oxy)ethyl) oxime (7ae). (172 mg, 68\%, 1:19 Z/E), yellow oil. ${ }^{1} \mathrm{H} \mathrm{NMR} \mathrm{(300} \mathrm{MHz,} \mathrm{CDCl}_{3}, 25$ $\left.{ }^{\circ} \mathrm{C}\right): \delta=0.70(\mathrm{~s}, 3 \mathrm{H}), 0.87-0.89(\mathrm{~d}, J=6.5 \mathrm{~Hz}, 6 \mathrm{H}), 0.92-0.95(\mathrm{~d}, J=6.4 \mathrm{~Hz}, 3 \mathrm{H}), 1.03(\mathrm{~s}, 3 \mathrm{H})$, 1.08-1.63 (overlapping signals, $23 \mathrm{H}), 1.81-2.09(\mathrm{~m}, 6 \mathrm{H}), 2.24-2.38$ (overlapping signals, $4 \mathrm{H}$ ), 3.44-3.52 (m, 1H), 3.65-3.76 (m, 1H), 5.27-5.40 (m, 1H), 5.52-5.58 (m, 1H), 7.37-7.38 (m, 3H), 7.66-7.70 (m, 2H); ${ }^{13} \mathrm{C}\left\{{ }^{1} \mathrm{H}\right\} \mathrm{NMR}\left(75.5 \mathrm{MHz}, \mathrm{CDCl}_{3}, 25^{\circ} \mathrm{C}\right): \delta=11.9,12.9(\mathrm{~d}, J=3.2 \mathrm{~Hz}), 15.3$, $18.7,19.4,20.9,21.1,22.6,22.8,23.8,24.3,28.0,28.2,28.7,29.6,31.9,35.8,36.2,36.7(\mathrm{~d}, J=$ $2.3 \mathrm{~Hz}), 37.3,37.4,39.3,38.5,39.8,40.2$, 42.3, 50.2, 56.2, 58.8, 98.3, $102.1102 .3,121.5(\mathrm{~d}, J=$ $7.6 \mathrm{~Hz}$ ), 126.1 (d, $J=2.6 \mathrm{~Hz}), 128.3,129.0,136.7,141.0,141.1$; HRMS (CI-DFS) $m / z:[\mathrm{M}+\mathrm{H}]^{+}$ Calcd for $\mathrm{C}_{37} \mathrm{H}_{58} \mathrm{NO}_{2}$ 548.4468; Found 548.4462.

(Z/E)-Acetophenone $O$-(1-(((1R,2S,5R)-2-isopropyl-5-methylcyclohexyl)oxy)ethyl) oxime (7af). (84 mg, 51\%, 1:3 Z/E), clear oil. ${ }^{1} \mathrm{H}$ NMR (300 MHz, $\left.\mathrm{CDCl}_{3}, 25{ }^{\circ} \mathrm{C}\right): \delta=0.66-0.69(\mathrm{~d}, J=7.2$ $\mathrm{Hz} ; 3 \mathrm{H}, E), 0.72-0.75(\mathrm{~d}, J=6.9 \mathrm{~Hz} ; 3 \mathrm{H}, Z$ ), 0.82-0.97 (overlapping signals, $13 \mathrm{H}, E$ and $Z$ ) 1.46$1.50(\mathrm{~d}, J=5.0 \mathrm{~Hz} ; 3 \mathrm{H}, E), 1.59-1.69$ (overlapping signals, $2 \mathrm{H}, E$ and $Z$ ), $2.26(\mathrm{~s}, 3 \mathrm{H}, E), 2.28$, (s, $3 \mathrm{H}, Z), 3.57-3.66$ (td, $J=10.5,4.8 \mathrm{~Hz} ; 1 \mathrm{H}, E), 3.82-3.90$ (td, $J=10.4,4.2 \mathrm{~Hz} ; 1 \mathrm{H}, Z), 5.43-5.48$ $(\mathrm{q}, J=5.3 \mathrm{~Hz} ; 1 \mathrm{H}, Z), 5.53-5.59(\mathrm{q}, J=5.5 \mathrm{~Hz} ; 1 \mathrm{H}, E), 7.35-7.41$ (overlapping signals, $3 \mathrm{H}, E$ and $Z$ ), 7.64-7.71 (overlapping signals, $2 \mathrm{H}, E$ and $Z$ ); ${ }^{13} \mathrm{C}\left\{{ }^{1} \mathrm{H}\right\} \mathrm{NMR}\left(75.5 \mathrm{MHz}, \mathrm{CDCl}_{3}, 25{ }^{\circ} \mathrm{C}\right): \delta=$ 12.9, 15.9, 21.1, 21.2, 22.3, 23.1, 25.1, 31.5, 34.4, 34.5, 40.1, 48.0, 48.9, 100.8, 126.0, 128.3, 129.0; HRMS (EI-DFS) $m / z$ : $[\mathrm{M}+\mathrm{H}]^{+}$Calcd for $\mathrm{C}_{20} \mathrm{H}_{31} \mathrm{NO}_{2}$ 317.2355; Found 317.2344.

(E)-Acetophenone $O$-(1-((6-chlorohexyl)oxy)ethyl) oxime (7ag). (130 mg, 88\%, 1:19 Z/E), clear oil. ${ }^{1} \mathrm{H}$ NMR $\left(300 \mathrm{MHz}, \mathrm{CDCl}_{3}, 25^{\circ} \mathrm{C}\right): \delta=1.37-1.52$ (overlapping signals, $\left.7 \mathrm{H}\right), 1.58-1.69(\mathrm{~m}$, 2H), 1.72-1.84 (m, 2H), $2.30(\mathrm{~s}, 3 \mathrm{H}), 3.49-3.54(\mathrm{t}, J=6.6 \mathrm{~Hz} ; 2 \mathrm{H}) 3.60-3.67(\mathrm{~m}, 1 \mathrm{H}), 3.83-3.92$ $(\mathrm{m}, 1 \mathrm{H}), 5.40-5.46(\mathrm{q}, J=5.4 \mathrm{~Hz} ; 1 \mathrm{H}), 7.38-7.42(\mathrm{~m}, 3 \mathrm{H}), 7.66-7.71(\mathrm{~m}, 2 \mathrm{H}) ;{ }^{13} \mathrm{C}\left\{{ }^{1} \mathrm{H}\right\} \mathrm{NMR}$ (75.5 MHz, $\left.\mathrm{CDCl}_{3}, 25^{\circ} \mathrm{C}\right): \delta=12.9,20.2,25.5,26.7,29.7,32.6,45.0,68.5,103.9,126.1,128.4$, 129.1, 136.7, 154.5; HRMS (EI-DFS) $m / z$ : $[\mathrm{M}+\mathrm{H}]^{+}$Calcd for $\mathrm{C}_{16} \mathrm{H}_{24} \mathrm{ClNO}_{2}$ 297.1496; Found 297.1492.

(E)-Acetophenone $O$-(1-(benzyloxy)ethyl) oxime (7ah). (87 mg, 65\%, 1:19 Z/E), clear oil. ${ }^{1} \mathrm{H}$ NMR $\left(300 \mathrm{MHz}, \mathrm{CDCl}_{3}, 25^{\circ} \mathrm{C}\right): \delta=1.54-1.65(\mathrm{~d}, J=5.5 \mathrm{~Hz} ; 3 \mathrm{H}), 2.29(\mathrm{~s}, 3 \mathrm{H}), 4.69-4.94(\mathrm{~m}$, 2H), 5.53-5.57 (q, $J=5.1 \mathrm{~Hz} ; 1 \mathrm{H}$ ), 7.29-7.43 (overlapping signals, 8H), 7.69-7.72 (m, 2H); ${ }^{13} \mathrm{C}\left\{{ }^{1} \mathrm{H}\right\} \mathrm{NMR}\left(75.5 \mathrm{MHz}, \mathrm{CDCl}_{3}, 25^{\circ} \mathrm{C}\right): \delta=12.9,20.3,70.4,103.5,126.1,127.5,127.8,128.3$, 128.4, 129.1; HRMS (EI-DFS) $m / z$ : $[\mathrm{M}+\mathrm{H}]^{+}$Calcd for $\mathrm{C}_{17} \mathrm{H}_{19} \mathrm{NO}_{2}$ 269.1416; Found 269.1530.

\section{Phase-transfer catalysis procedure:}

\section{1-(Fluoromethyl)-4-nitrobenzene $(8){ }^{20}$}

To an oven-dried $10.0 \mathrm{~mL}$ round-bottom flask charged with $4 \mathbf{4 e} \cdot \mathrm{BF}_{4}^{-}(20 \mathrm{mg}, 10 \mathrm{~mol} \%)$; 4nitrobenzyl bromide ( $85 \mathrm{mg} 0.4 \mathrm{mmol})$ and cesium fluoride $(182 \mathrm{mg}, 1.20 \mathrm{mmol})$ were combined and subsequently diluted in propionitrile $(2 \mathrm{~mL})$. The resulting mixture was stirred for $5 \mathrm{~h}$ at 65 ${ }^{\circ} \mathrm{C}$ under an inert atmosphere. After 5 hours, a second portion of $4 \mathbf{e} \cdot \mathrm{BF}_{4}^{-}(20 \mathrm{mg}, 10 \mathrm{~mol} \%)$ was added and stirred for a further 5 hours under the same conditions. Reaction progress was monitored using ${ }^{1} \mathrm{H}$ NMR. After removal of the solvent, the crude material was subjected to flash chromatography (6:1 Hexane/EtOAc) to furnish the product as a yellow oil $(51 \mathrm{mg}, 83 \%) .{ }^{1} \mathrm{H}$ 
$\operatorname{NMR}\left(300 \mathrm{MHz}, \mathrm{CDCl}_{3}, 25{ }^{\circ} \mathrm{C}\right): \delta=5.45-5.61(\mathrm{~d}, J=47.1 \mathrm{~Hz} ; 2 \mathrm{H}), 7.53-7.57(\mathrm{~d}, J=8.4 \mathrm{~Hz}$; $2 \mathrm{H}), 8.26-8.30$ (d, $J=8.4 \mathrm{~Hz} ; 2 \mathrm{H})$.

\section{Alkylation procedure:}

\section{2,4-Dichloro- $N$-(1-phenylethyl)aniline (9). ${ }^{21}$}

To an oven-dried $10.0 \mathrm{~mL}$ round-bottom flask charged with $4 \mathrm{e}^{-\mathrm{BF}_{4}^{-}}(24 \mathrm{mg}, 10 \mathrm{~mol} \%)$; 1 phenylethyl-2,2,2-trichloroacetimidate $(100 \mathrm{mg}, 0.38 \mathrm{mmol})$ and 2,4-dichloroaniline $(91 \mathrm{mg}, 0.56$ $\mathrm{mmol})$ were combined and subsequently diluted in dichloromethane $(2 \mathrm{~mL})$. The resulting solution was stirred for $4 \mathrm{~h}$ at room temperature under an inert atmosphere. Reaction progress was monitored via TLC. After removal of the solvent, the crude material was subjected to flash chromatography $\left(80: 16: 4 \mathrm{Hexane} / \mathrm{DCM} / \mathrm{NEt}_{3}\right)$ to furnish the product as a colourless oil (74 $\mathrm{mg}$, $73 \%) .{ }^{1} \mathrm{H} \mathrm{NMR}\left(400 \mathrm{MHz}, \mathrm{CDCl}_{3}, 25^{\circ} \mathrm{C}\right): \delta=1.59-1.61(\mathrm{~d}, J=6.4 \mathrm{~Hz} ; 3 \mathrm{H}), 4.46-4.56(\mathrm{~m}, 1 \mathrm{H})$, 4.70 (brs, 1H) 6.32-6.35 (d, $J=9.0 \mathrm{~Hz} ; 1 \mathrm{H}), 6.92-6.95(\mathrm{dd}, J=6.1 \mathrm{~Hz}, 2.6 \mathrm{~Hz} ; 1 \mathrm{H}), 7.24-7.39$ $(\mathrm{m}, 6 \mathrm{H})$.

\section{Etherification procedure:}

\section{Benzyl-1-Phenylethyl Ether (10). ${ }^{22}$}

To an oven-dried $10.0 \mathrm{~mL}$ round-bottom flask charged with $4 \mathrm{e}^{-\mathrm{BF}_{4}{ }^{-}}(24 \mathrm{mg}, 10 \mathrm{~mol} \%)$; 1 phenylethyl-2,2,2-trichloroacetimidate $(100 \mathrm{mg} 0.38 \mathrm{mmol})$ and benzyl alcohol $(61 \mathrm{mg}, 0.56$ $\mathrm{mmol})$ were combined and subsequently diluted in dichloromethane $(2 \mathrm{~mL})$. The resulting solution was stirred for $2 \mathrm{~h}$ at room temperature under an inert atmosphere. Reaction progress was monitored via TLC. After removal of the solvent, the crude material was subjected to flash chromatography (4:1 Hexane/EtOAc) to furnish the product as a colourless oil $(71 \mathrm{mg}, 88 \%) .{ }^{1} \mathrm{H}$ NMR $\left(400 \mathrm{MHz}, \mathrm{CDCl}_{3}, 25^{\circ} \mathrm{C}\right): \delta=1.55-1.56(\mathrm{~d}, J=6.4 \mathrm{~Hz} ; 3 \mathrm{H}), 4.35-4.38(\mathrm{~d}, J=11.4 \mathrm{~Hz}$; $1 \mathrm{H}), 4.51-4.54(\mathrm{~d}, J=12.1 \mathrm{~Hz} ; 1 \mathrm{H}) 4.55-4.60(\mathrm{q}, J=6.4 \mathrm{~Hz} ; 1 \mathrm{H}), 7.33-7.46(\mathrm{~m}, 10 \mathrm{H})$.

\section{Glycosylation procedure:}

\section{Benzyl 2,3,4,6-tetra- $\boldsymbol{O}$-acetyl- $\boldsymbol{\beta}$-D-galactopyranoside (11). ${ }^{23}$}

To an oven-dried $10.0 \mathrm{~mL}$ round-bottom flask charged with $4 \mathbf{e} \cdot \mathrm{BF}_{4}^{-}(15 \mathrm{mg}, 10 \mathrm{~mol} \%)$; 2,3,4,6-tetra- $O$ - $\alpha$-D-galactopyranosyl trichloroacetimidate $(100 \mathrm{mg} 0.20 \mathrm{mmol}$ ) and benzyl alcohol (32 mg, $0.30 \mathrm{mmol}$ ) were combined and subsequently diluted in dichloromethane $(2 \mathrm{~mL})$. The resulting solution was stirred for $5 \mathrm{~h}$ at room temperature under an inert atmosphere. Reaction progress was monitored via TLC. After removal of the solvent, the crude material was subjected to flash chromatography (2:1 Hexane/EtOAc) to furnish the product as a colourless oil (75 $\mathrm{mg}$, 86\%). ${ }^{1} \mathrm{H}$ NMR $\left(300 \mathrm{MHz}, \mathrm{CDCl}_{3}, 25{ }^{\circ} \mathrm{C}\right): \delta=2.00(\mathrm{~s}, 3 \mathrm{H}), 2.04(\mathrm{~s}, 3 \mathrm{H}), 2.09(\mathrm{~s}, 3 \mathrm{H}), 2.18(\mathrm{~s}$, $3 \mathrm{H}), 3.88-3.94(\mathrm{td}, J=6.7,1.0 \mathrm{~Hz} ; 1 \mathrm{H}), 4.13-4.28(\mathrm{~m}, 1 \mathrm{H}), 4.52-4.56(\mathrm{~d}, J=8.0 \mathrm{~Hz} ; 1 \mathrm{H}), 4.63-$ $4.68(\mathrm{~d}, J=12.5 \mathrm{~Hz} ; 1 \mathrm{H}), 4.91-4.97(\mathrm{~d}, J=13.2 \mathrm{~Hz} ; 1 \mathrm{H}), 4.99-5.01$ (d, $J=3.8 \mathrm{~Hz} ; 1 \mathrm{H}), 5.02-$ $5.04(\mathrm{~d}, J=3.5 \mathrm{~Hz} ; 1 \mathrm{H}), 5.28-5.30(\mathrm{dd}, J=8.0,2.6 \mathrm{~Hz} ; 1 \mathrm{H}), 5.39-5.41$ (dd, $J=2.4,0.8 \mathrm{~Hz} ; 1 \mathrm{H})$, 7.29-7.41 (m, 5H). 
Table S5. Control reactions in the absence of catalyst $4 \mathbf{e} \cdot \mathrm{BF}_{4}{ }^{-}$.

\begin{tabular}{ccc}
\hline $\begin{array}{c}\text { control reaction conditions } \\
\text { of applications }\end{array}$ & time (h) & $\begin{array}{c}\text { conversion (\%) } \\
(\mathbf{8}-\mathbf{1 1})\end{array}$ \\
\hline Phase Transfer & 10 & 5 \\
Alkylation & 5 & No Conversion \\
Etherification & 5 & No Conversion \\
Glycosylation & 10 & No Conversion
\end{tabular}




\section{Spectra}

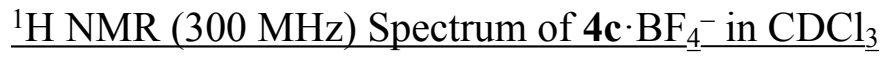
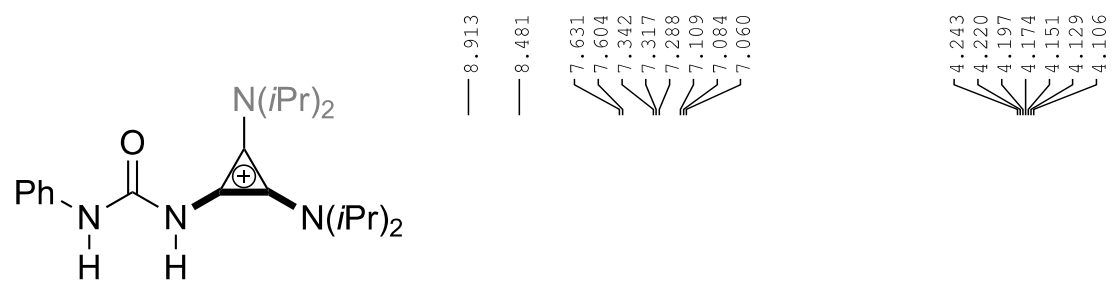

V)

$\mathrm{BF}_{4}^{-}$

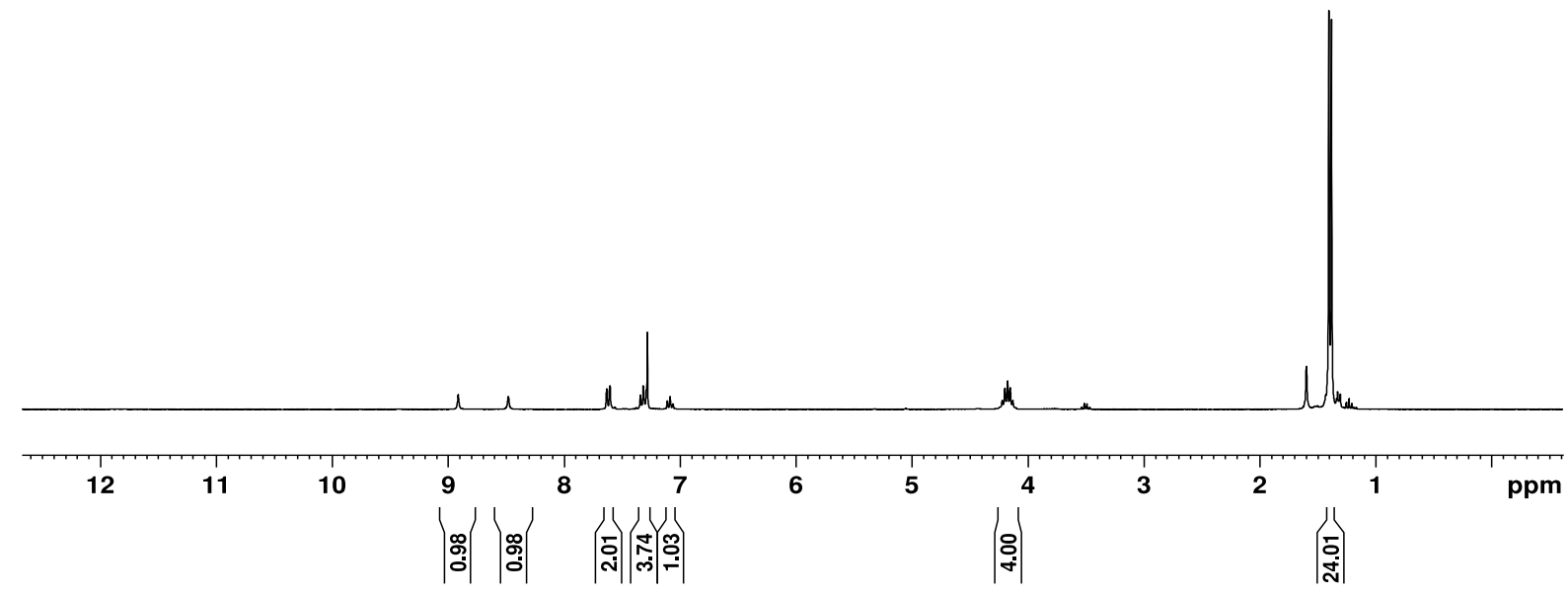

${ }^{13} \mathrm{C}\left\{{ }^{1} \mathrm{H}\right\}$ NMR (75.5 MHz) Spectrum of $4 \mathbf{c} \cdot \mathrm{BF}_{4}{ }^{-}$in $\mathrm{CDCl}_{3}$
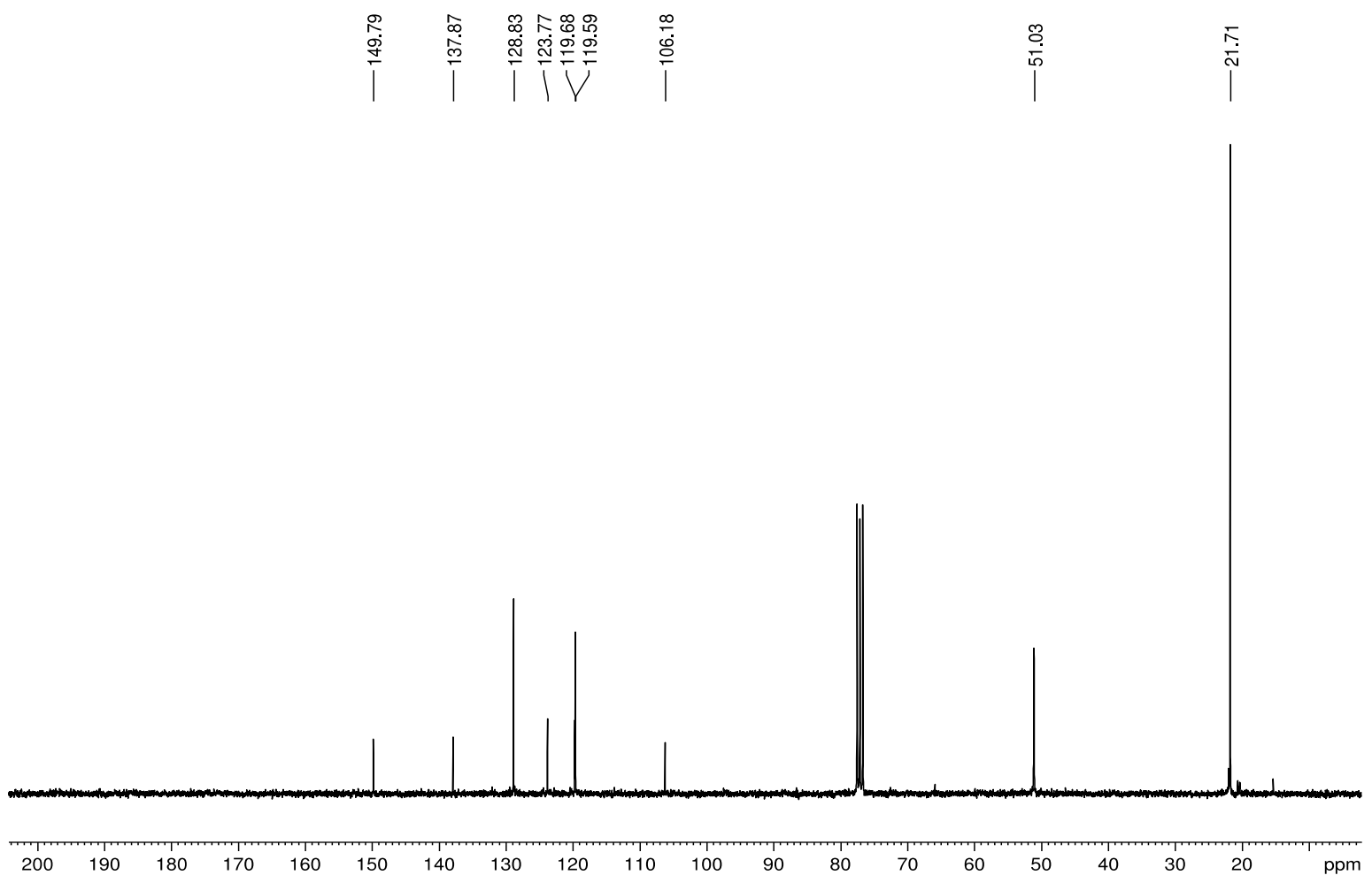


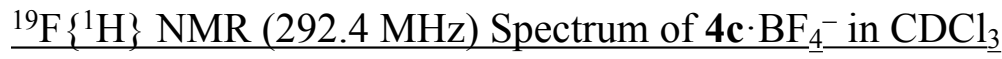

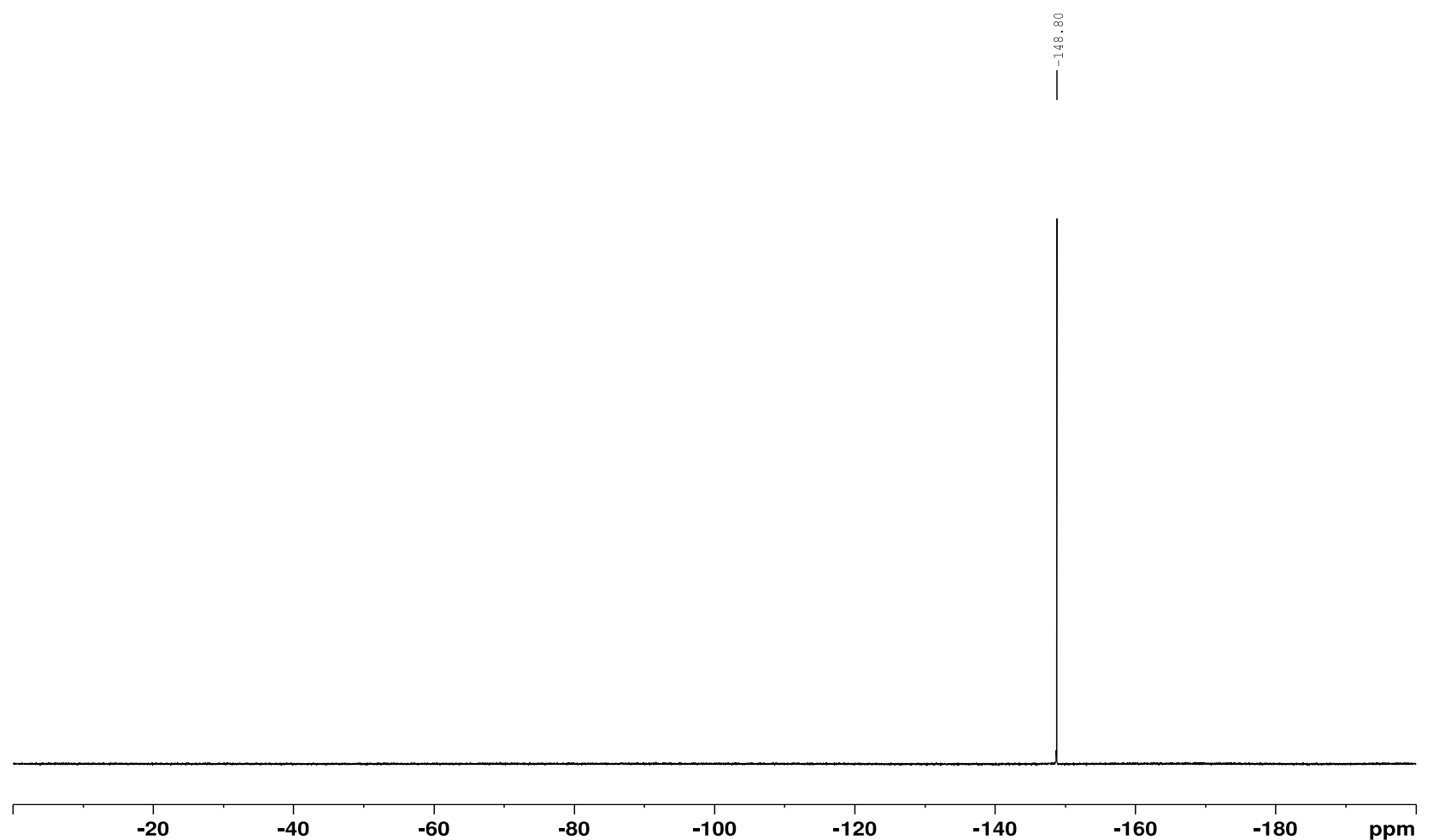

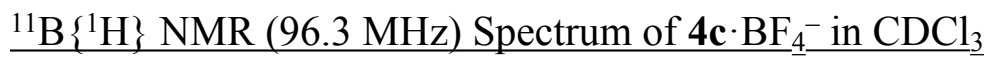

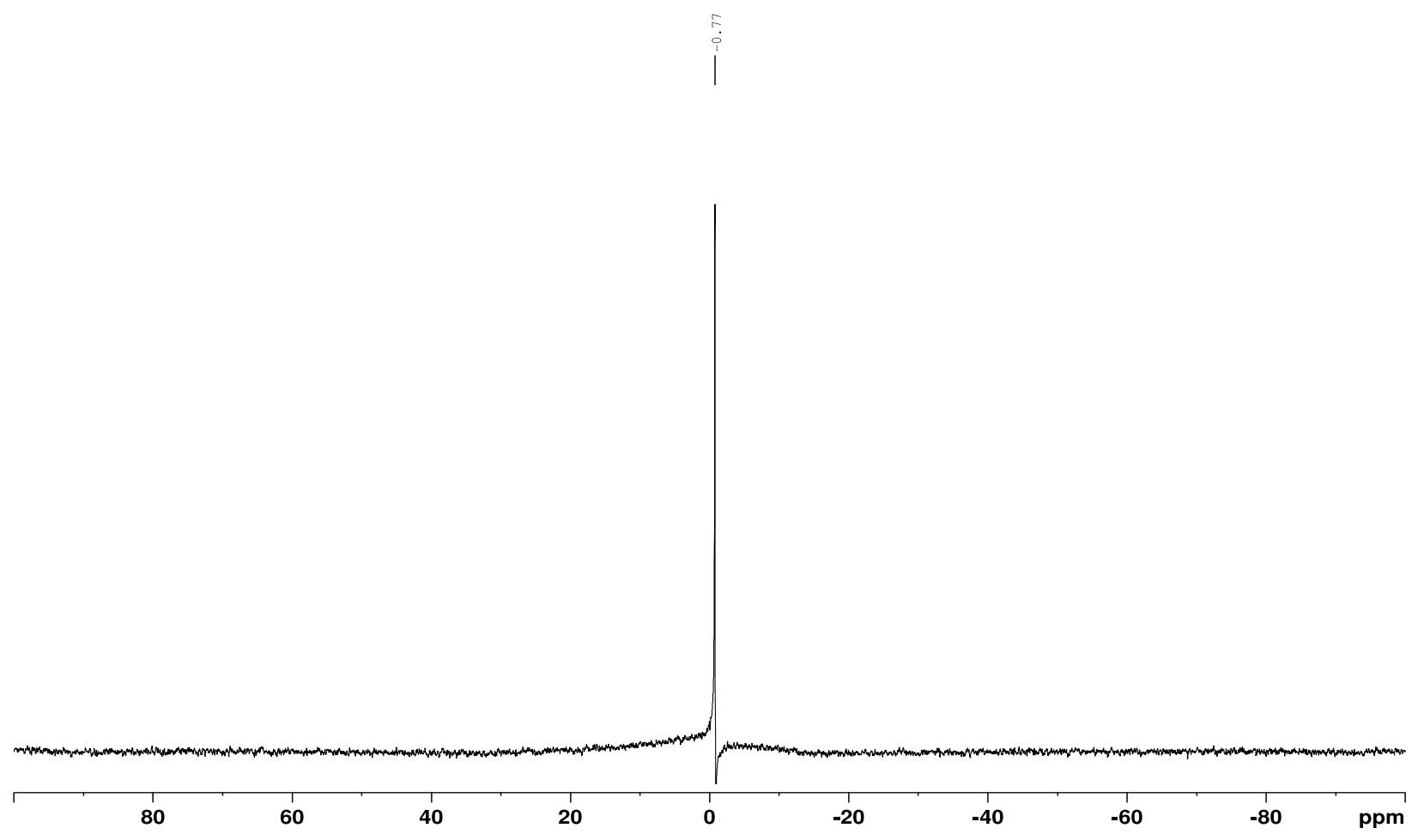


$\underline{{ }^{1} \mathrm{H} \text { NMR (300 MHz) Spectrum of } \mathbf{3} \text { in } \mathrm{CDCl}_{3}}$

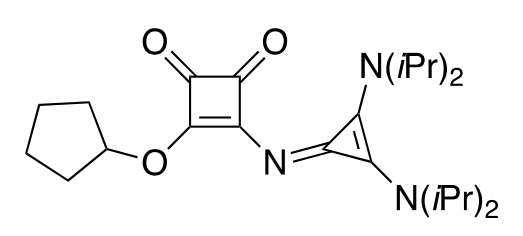

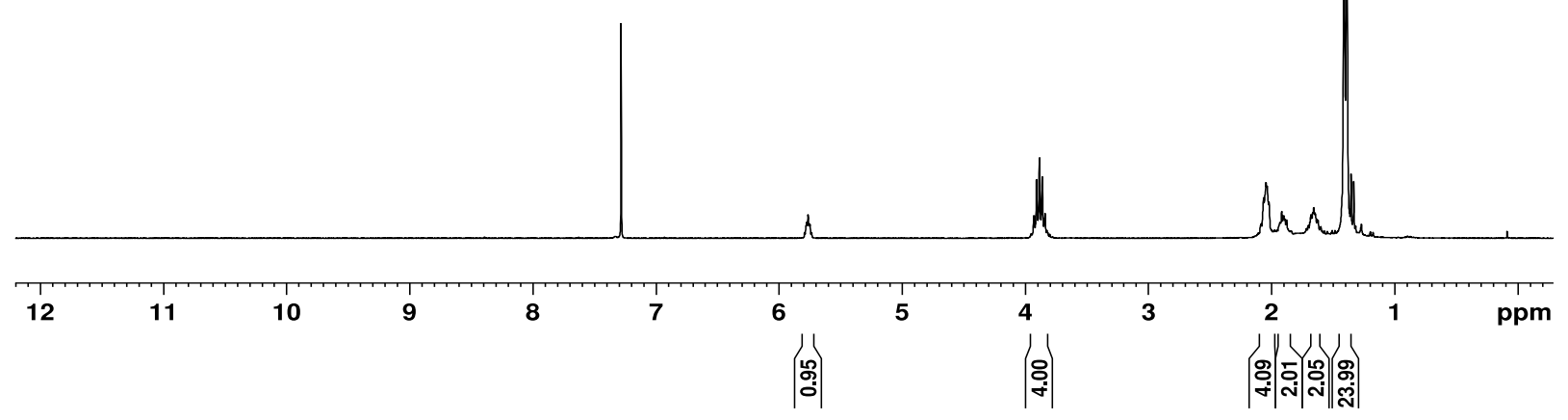

${ }^{13} \mathrm{C}\left\{{ }^{1} \mathrm{H}\right\}$ NMR (75.5 MHz) Spectrum of $\mathbf{3}$ in $\mathrm{CDCl}_{3}$

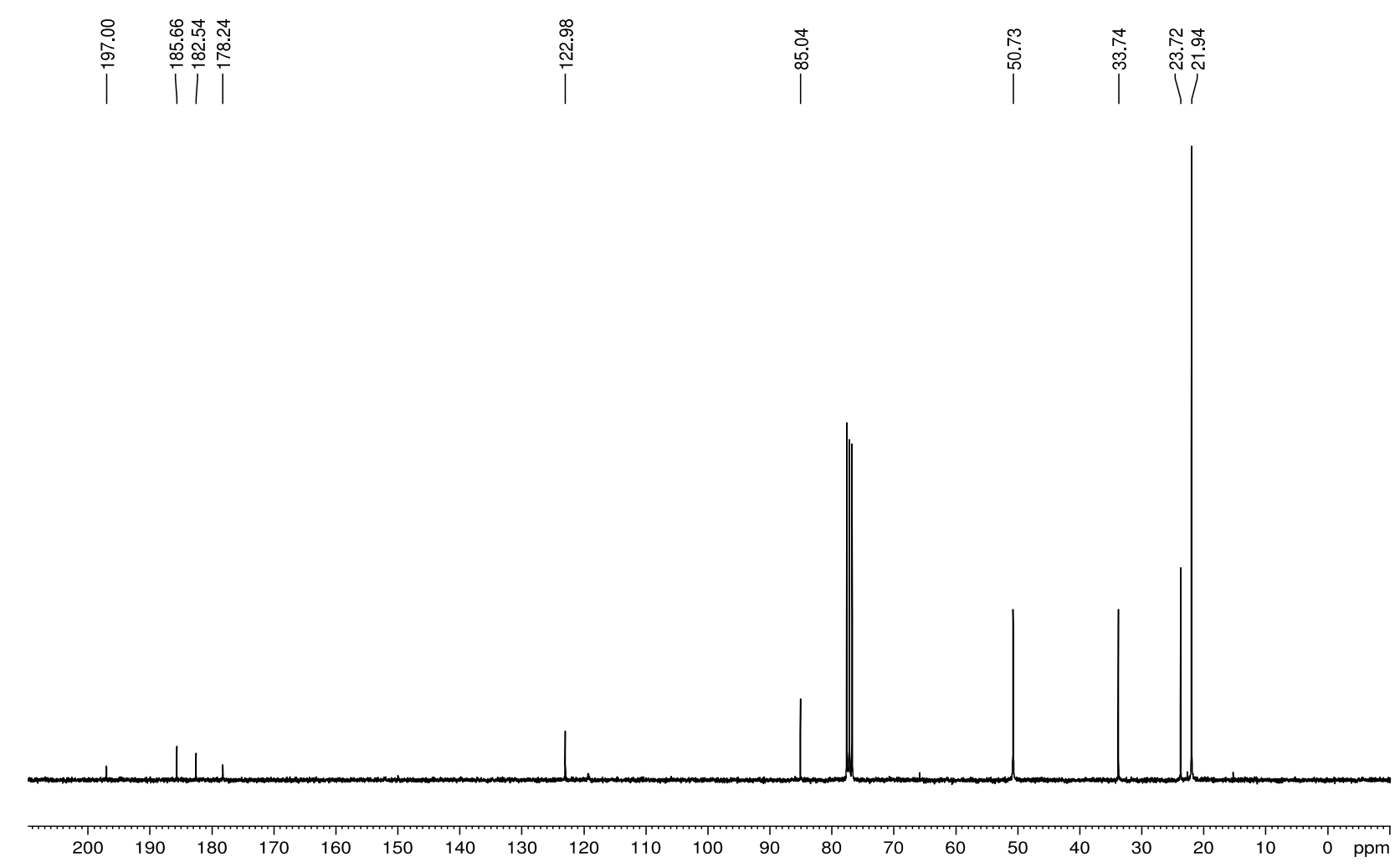




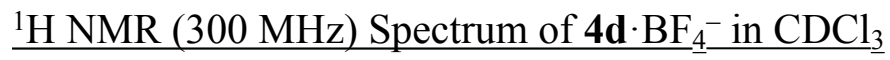

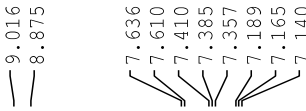

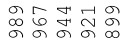

$\sqrt[\text { inim }]{\min }$

Viv<smiles></smiles>

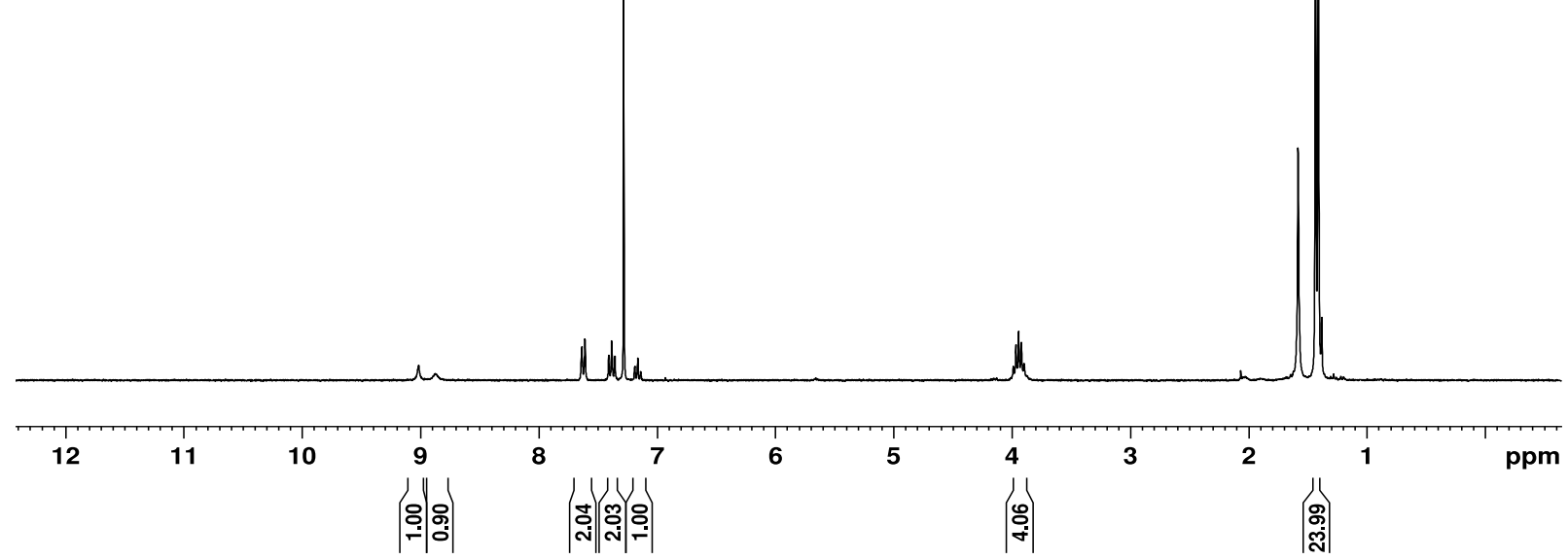

${ }^{13} \mathrm{C}\left\{{ }^{1} \mathrm{H}\right\}$ NMR (75.5 MHz) Spectrum of $\mathbf{4 d} \cdot \mathrm{BF}_{4}^{-}$in $\mathrm{CDCl}_{3}$

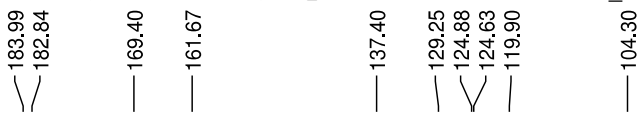

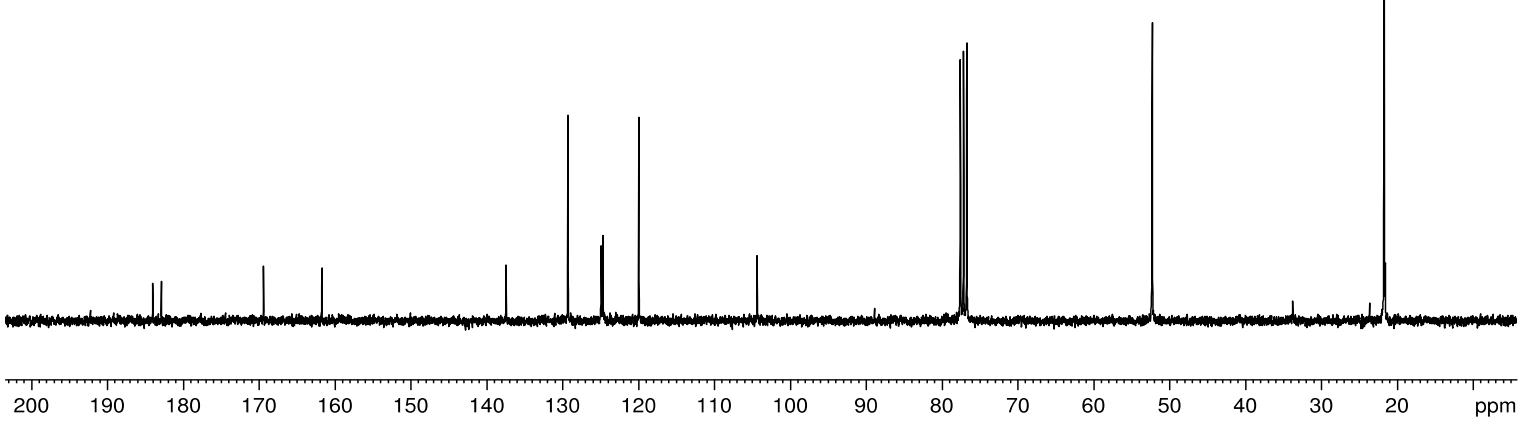




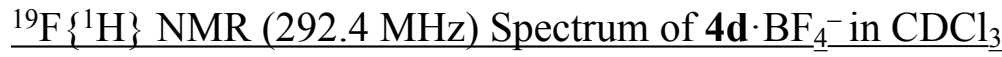

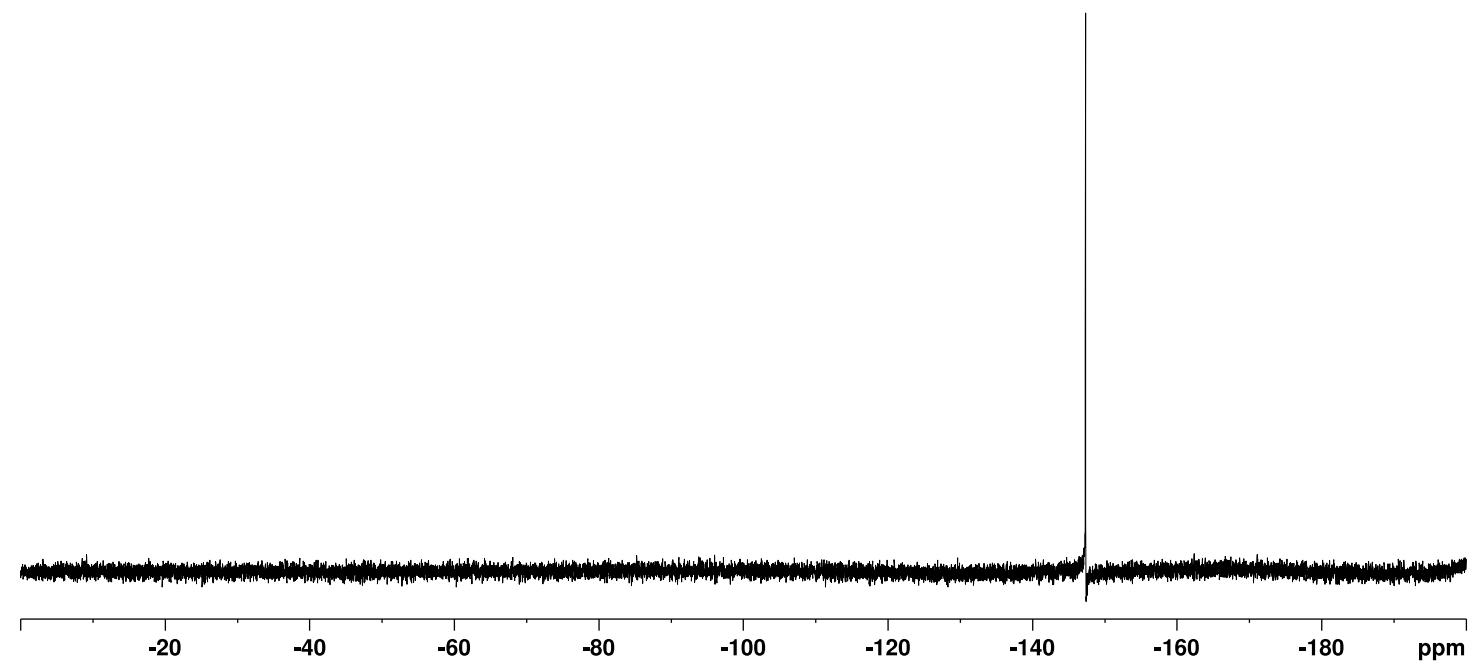

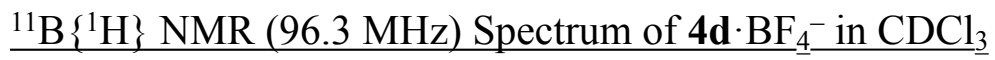

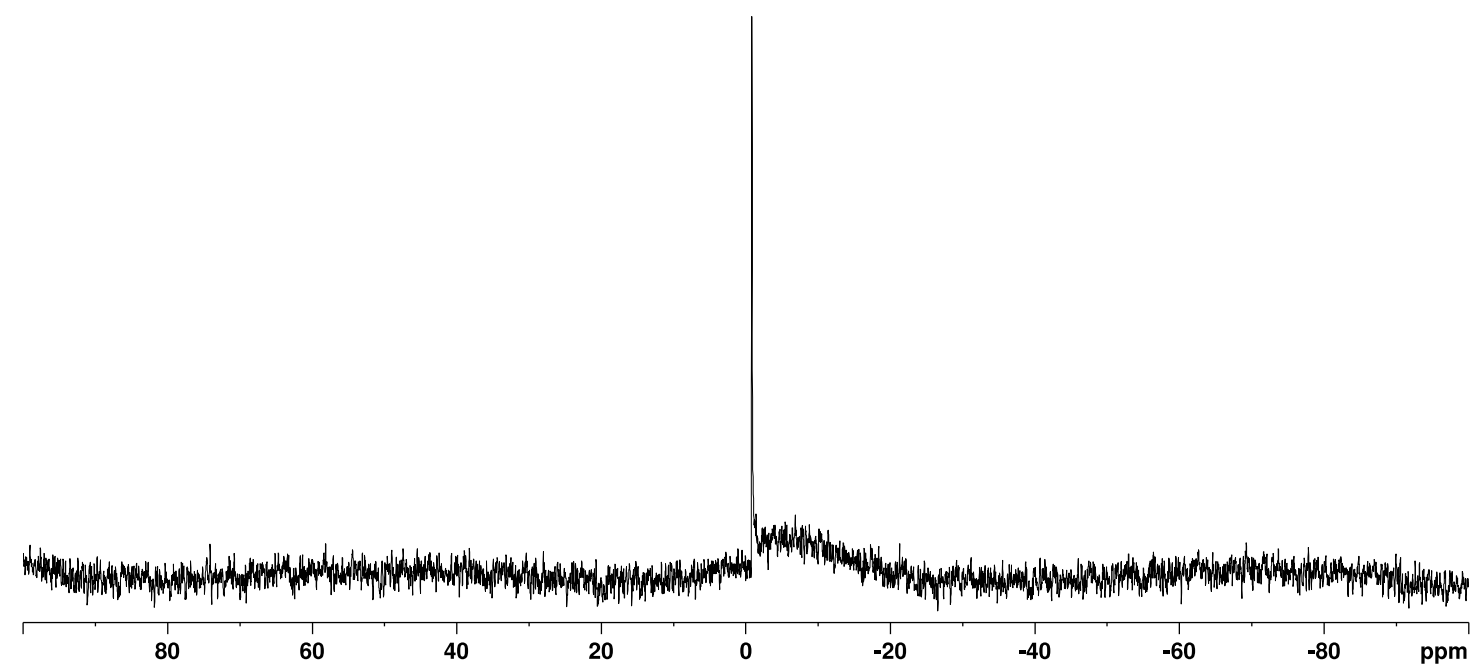




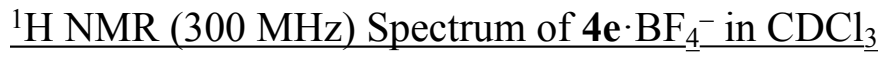

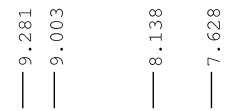<smiles></smiles>

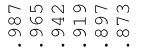

نंiniving

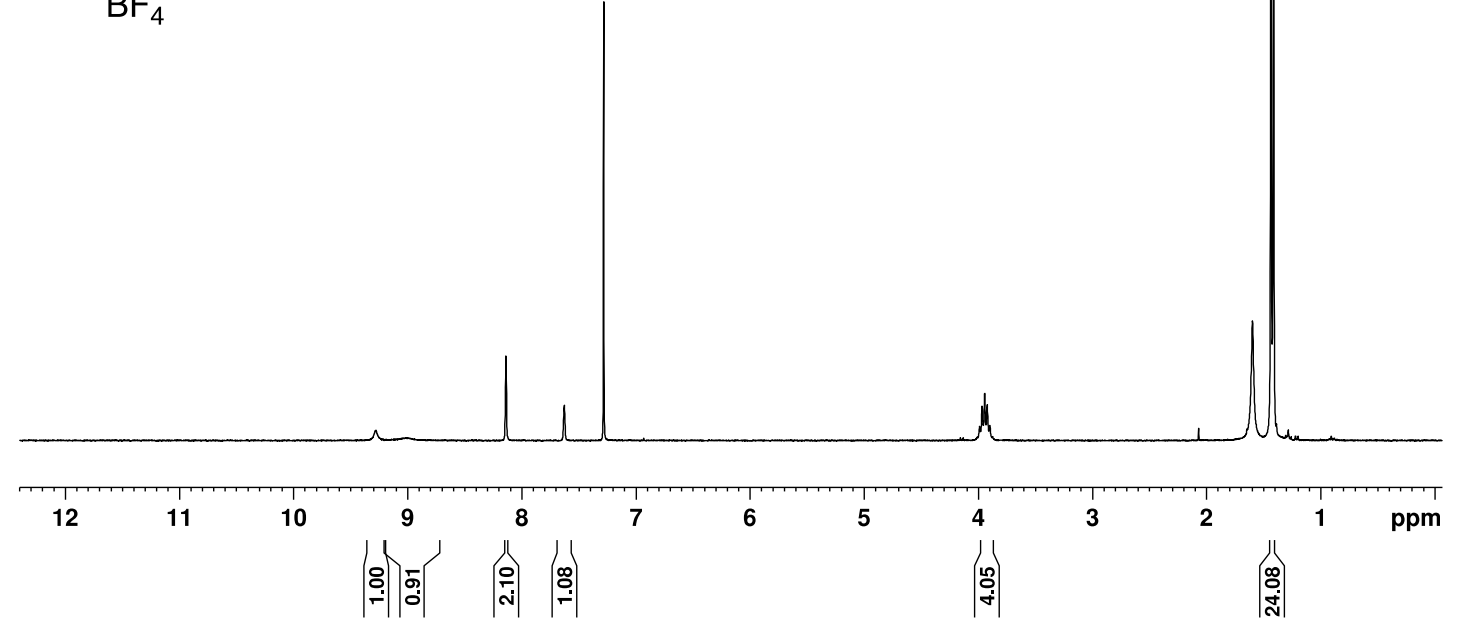

${ }^{13} \mathrm{C}\left\{{ }^{1} \mathrm{H}\right\}$ NMR (75.5 MHz) Spectrum of $4 \mathrm{e} \cdot \mathrm{BF}_{4}^{-}{ }^{-}$in $\mathrm{CDCl}_{3}$

V | |
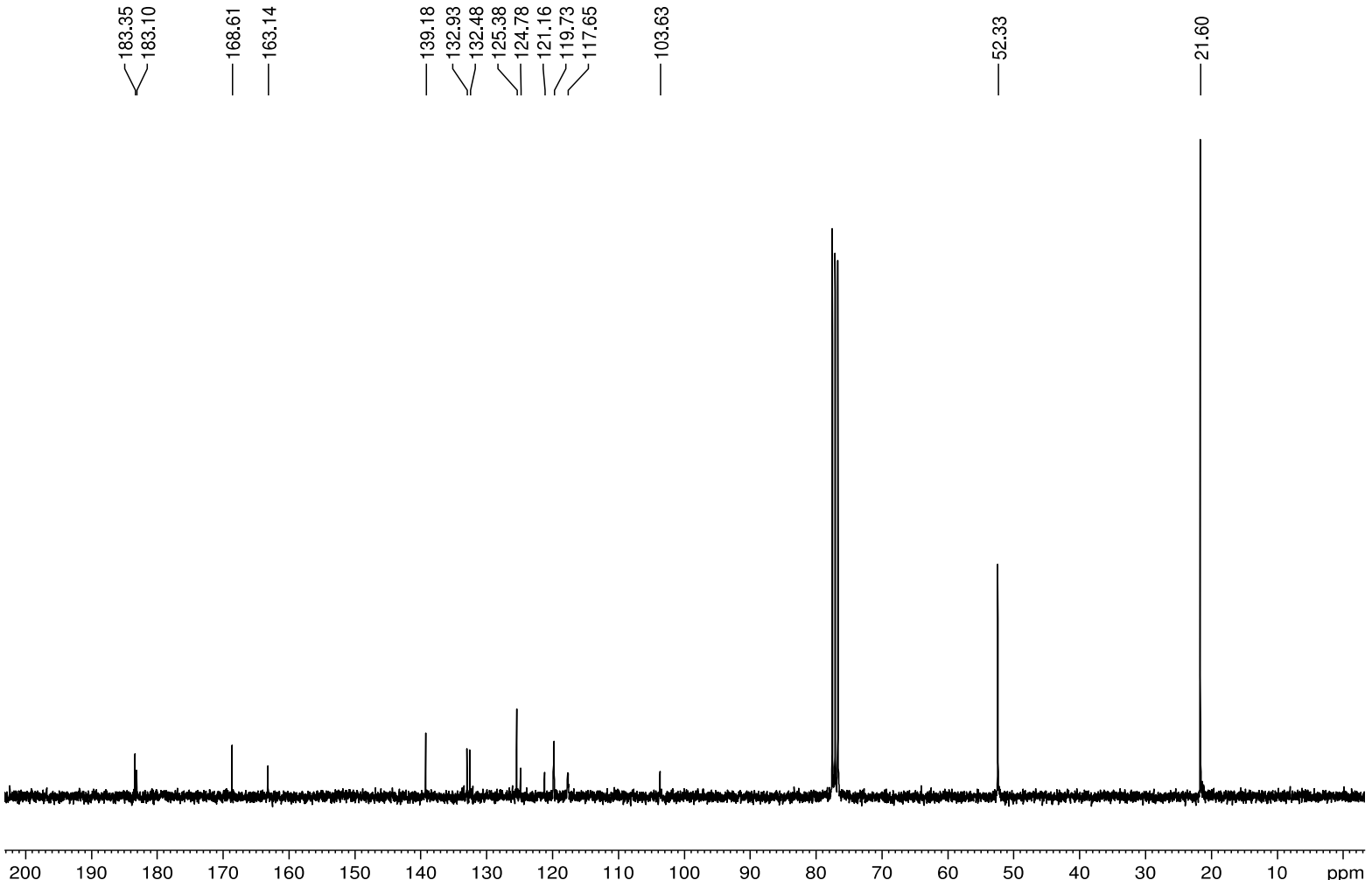


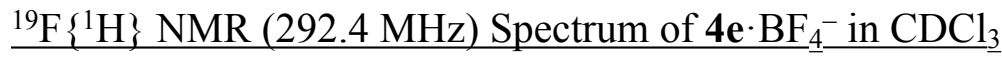

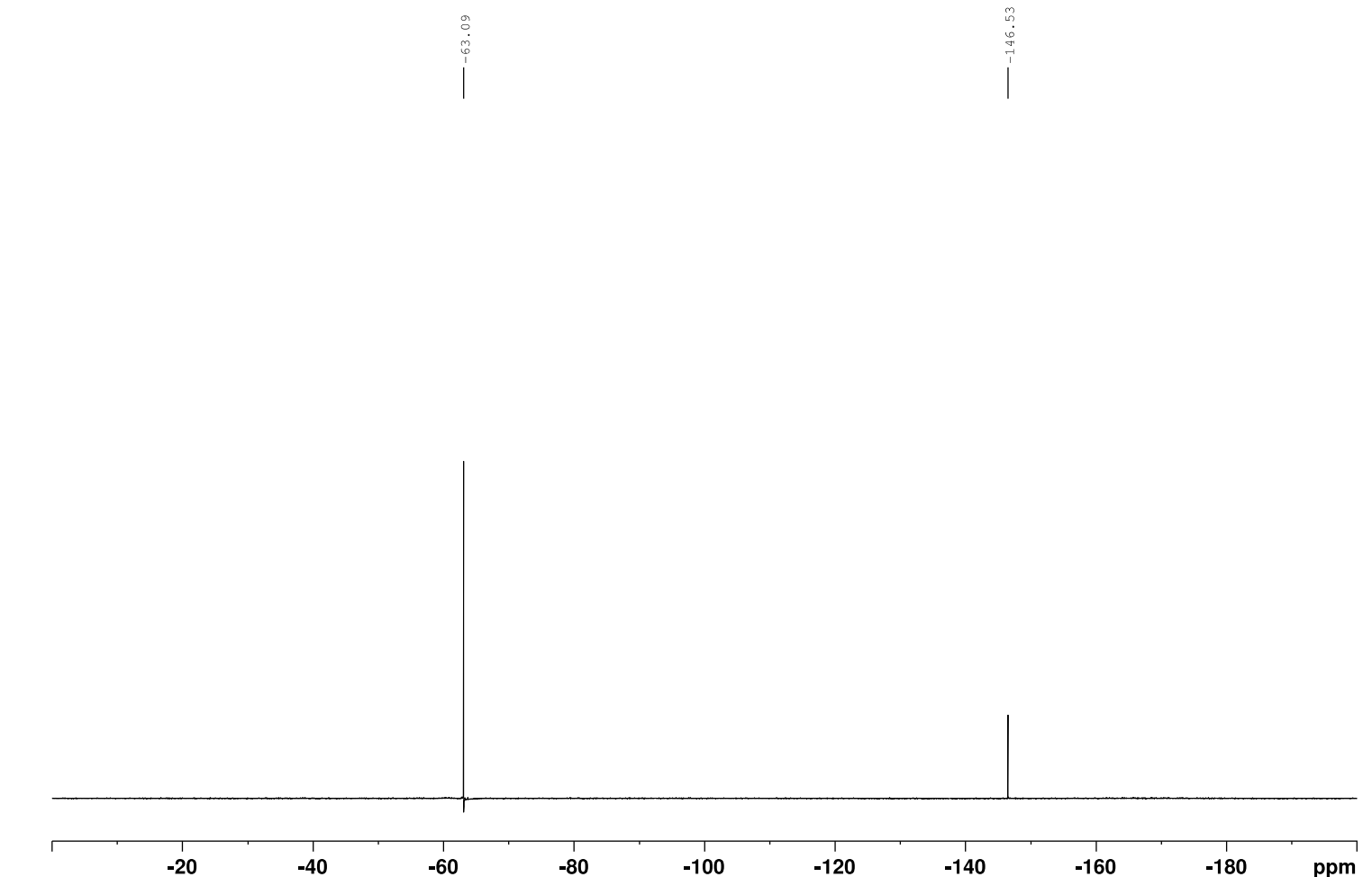

$\underline{{ }^{11} \mathrm{~B}\left\{{ }^{1} \mathrm{H}\right\} \text { NMR (96.3 MHz) Spectrum of } 4 \mathrm{e} \cdot \mathrm{BF}_{4}^{-}{ }^{-} \text {in } \mathrm{CDCl}_{3}}$

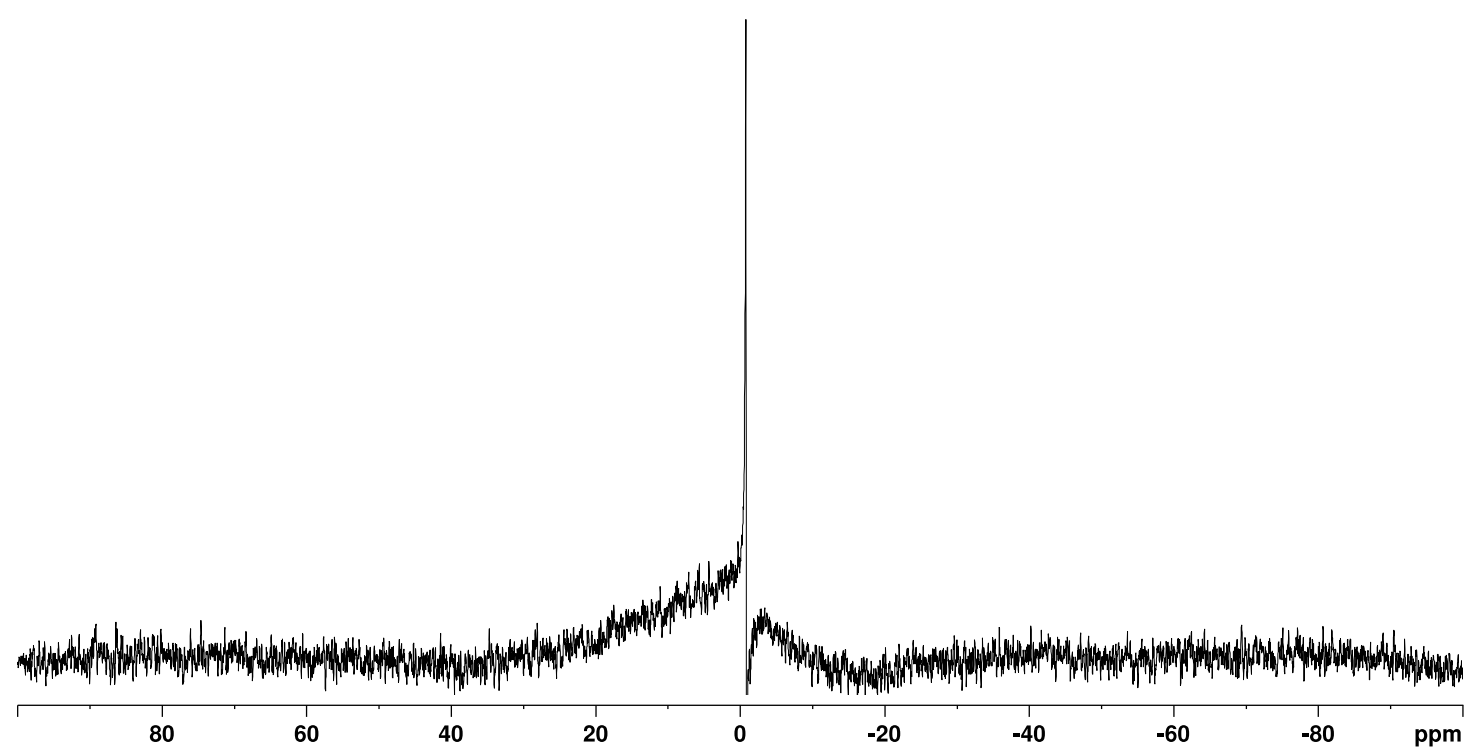


$\underline{{ }^{1} \mathrm{H} \text { NMR (300 MHz) Spectrum of } \mathbf{S 1} \text { in } \mathrm{CDCl}_{3}}$
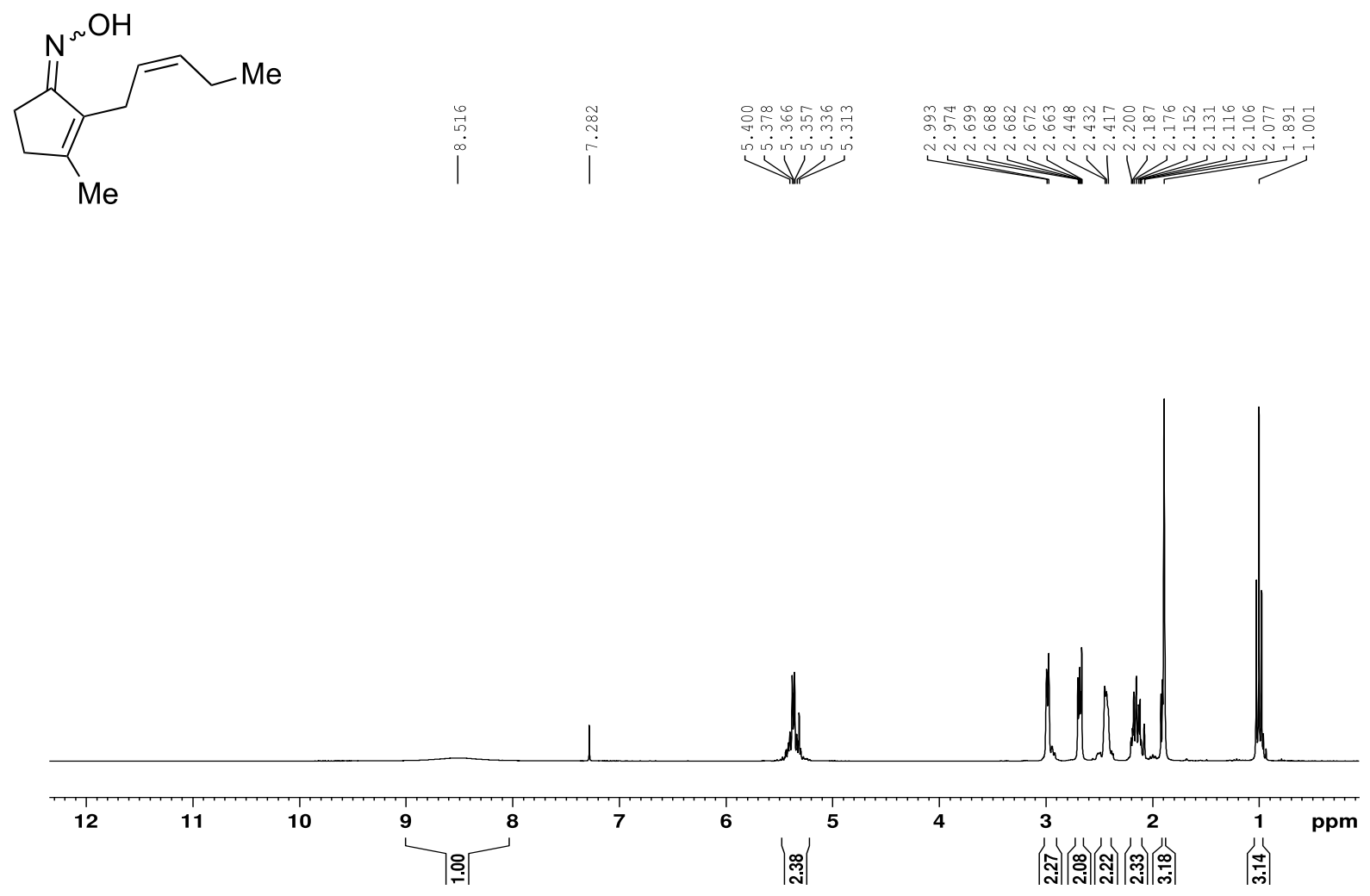

${ }^{13} \mathrm{C}\left\{{ }^{1} \mathrm{H}\right\}$ NMR (75.5 MHz) Spectrum of $\mathbf{S 1}$ in $\mathrm{CDCl}_{3}$

|

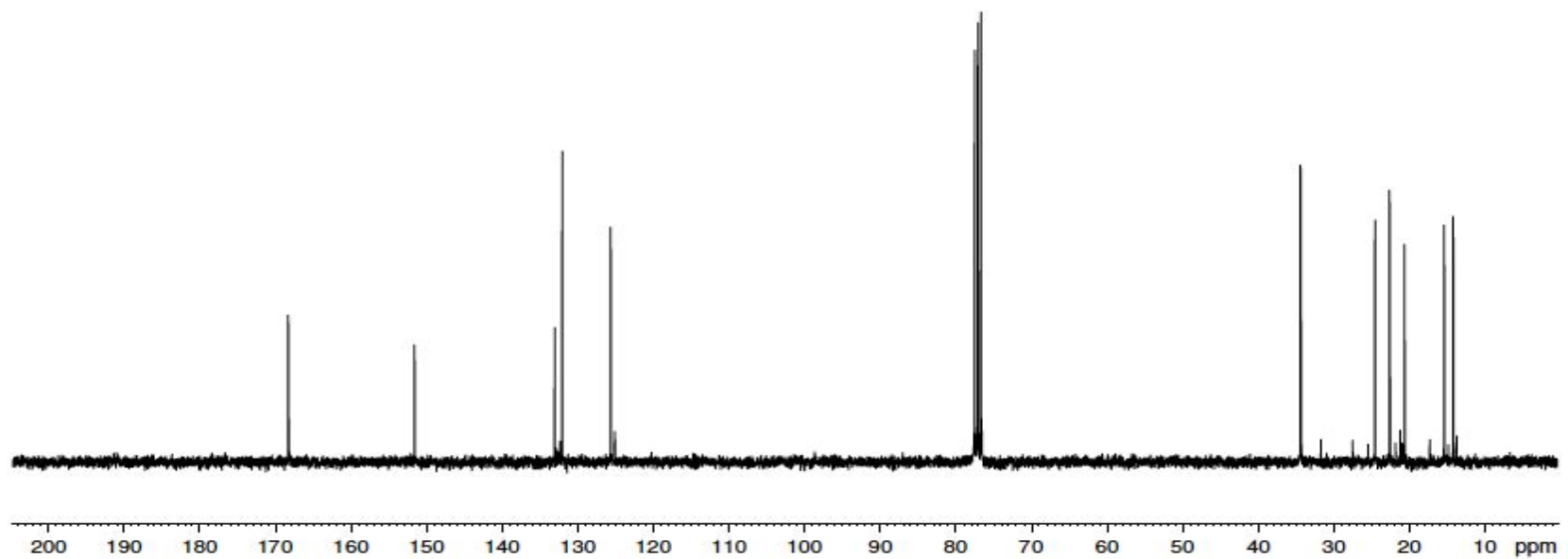


$\underline{{ }^{1} \mathrm{H} \text { NMR (300 MHz) Spectrum of } \mathbf{S 2} \text { in } \mathrm{CDCl}_{3}}$<smiles>CC(Cc1ccc(C(C)(C)C)cc1)/C([N])=N/O</smiles>

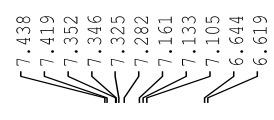

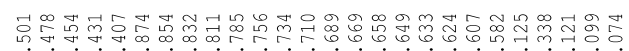

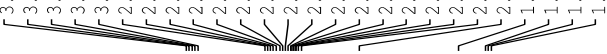

\footnotetext{
t-Bu
}

$$
\text { (n) }
$$

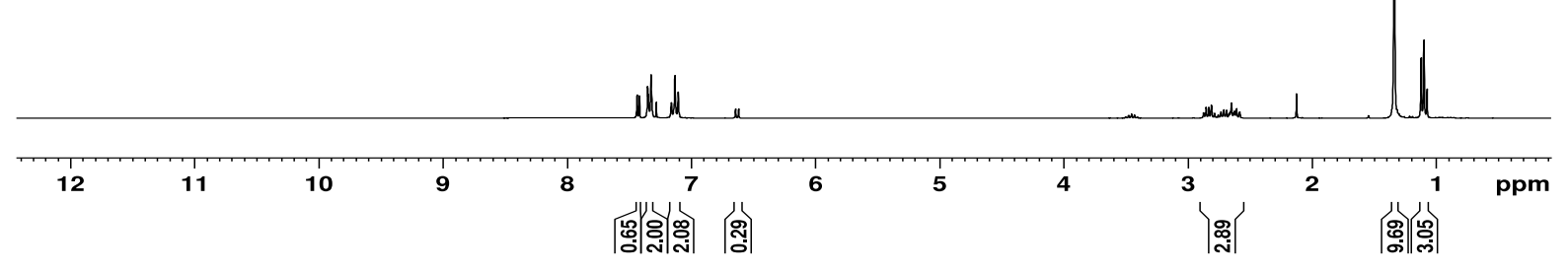

${ }^{13} \mathrm{C}\left\{{ }^{1} \mathrm{H}\right\}$ NMR (75.5 MHz) Spectrum of $\mathbf{S 2}$ in $\mathrm{CDCl}_{3}$
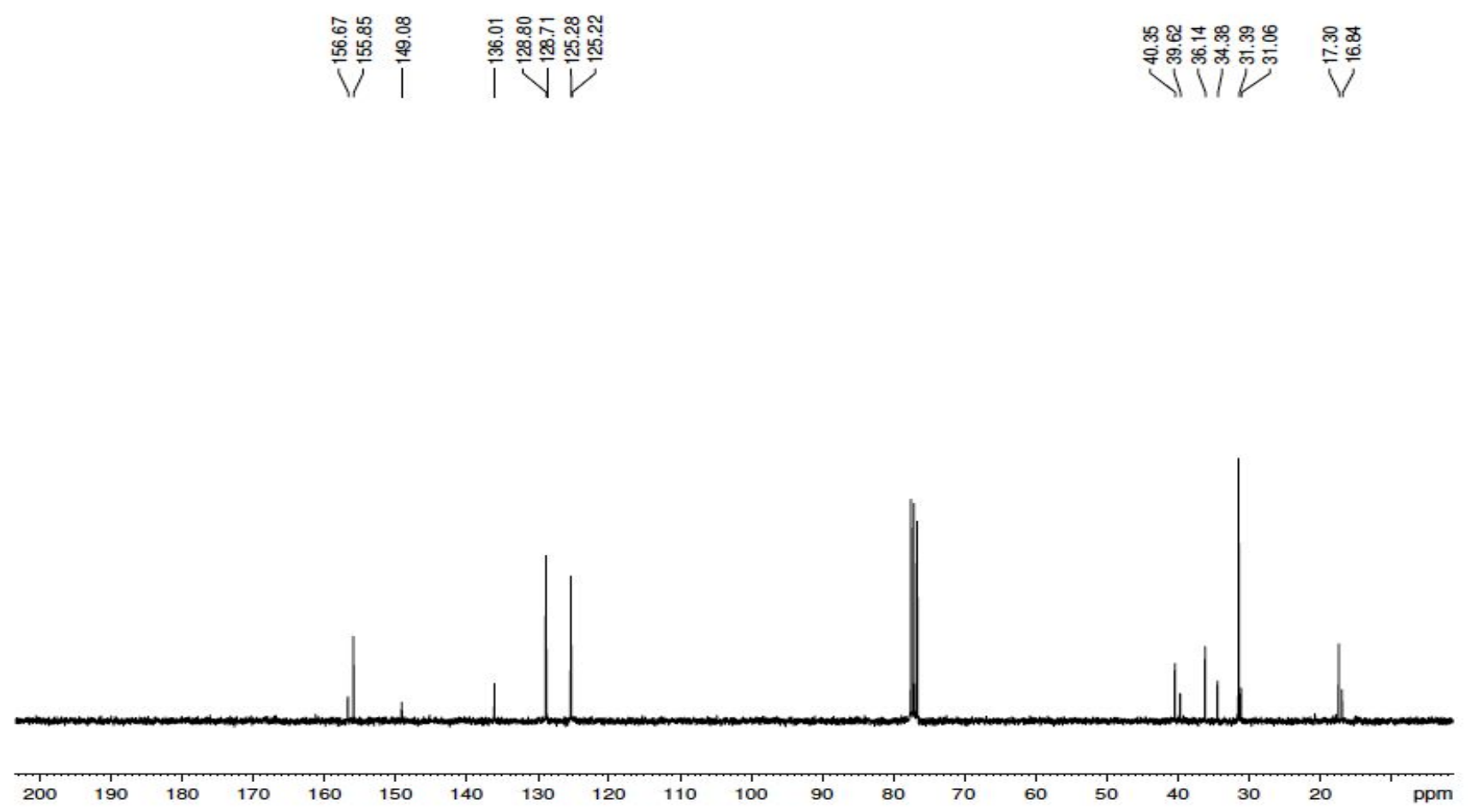
$\underline{{ }^{1} \mathrm{H} N M R}$ (300 MHz) Spectrum of $\mathbf{S 3}$ in $\mathrm{CDCl}_{3}$

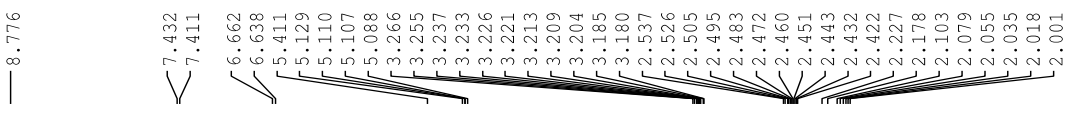<smiles>CC(C)=CCCC1=CCC(/C=N/O)CC1</smiles>

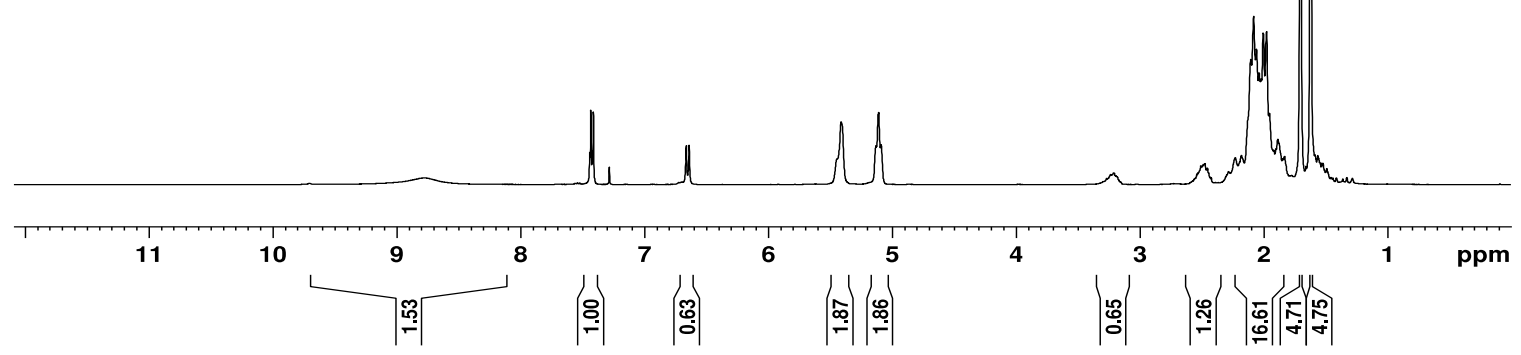

${ }^{13} \mathrm{C}\left\{{ }^{1} \mathrm{H}\right\}$ NMR (75.5 MHz) Spectrum of $\mathbf{S 3}$ in $\mathrm{CDCl}_{3}$

$\left.\right|_{\mid} ^{\infty}$

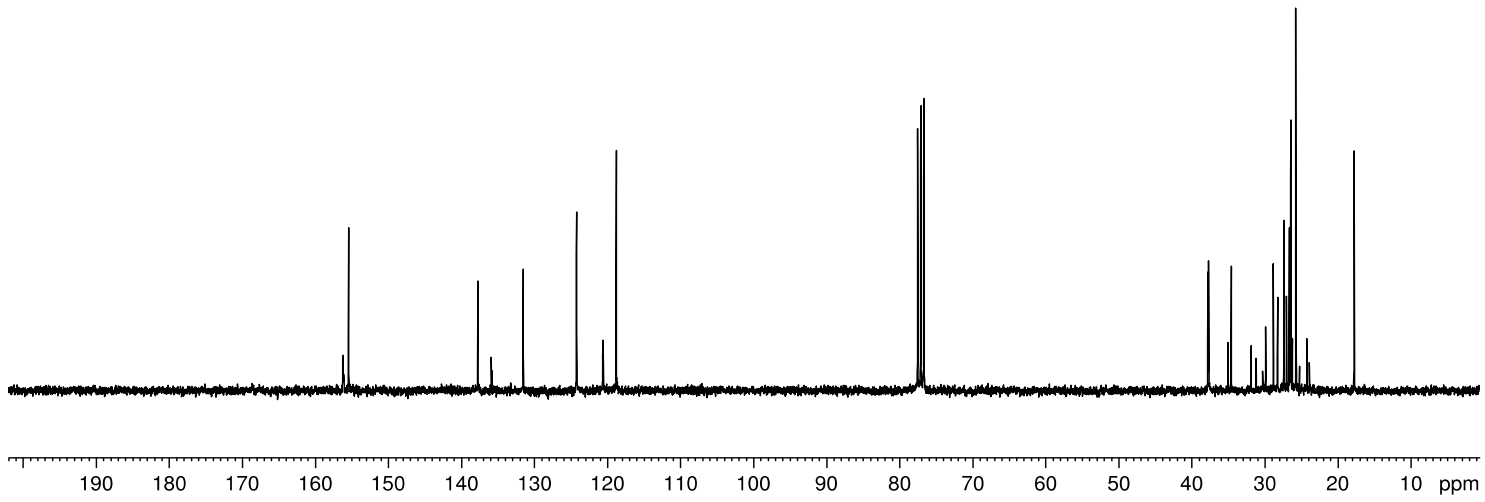


$\underline{{ }^{1} \mathrm{H} \text { NMR }(300 \mathrm{MHz}) \text { Spectrum of a 1:3 Z/E mixture of 7a in } \mathrm{CDCl}_{3}}$

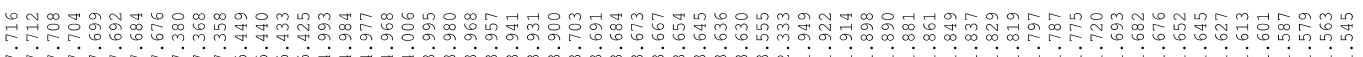

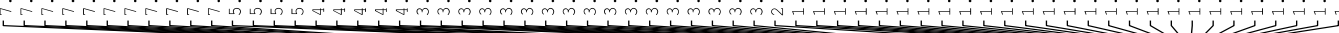<smiles>C/C(=N\OC1CCCCO1)c1ccccc1</smiles>

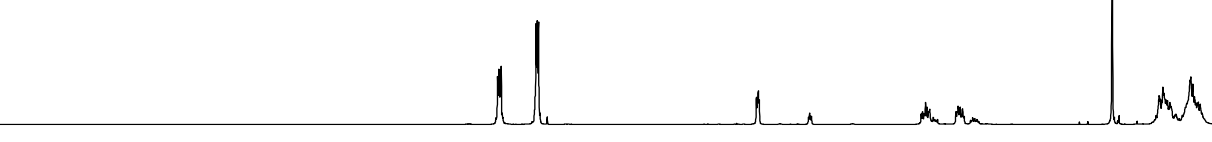

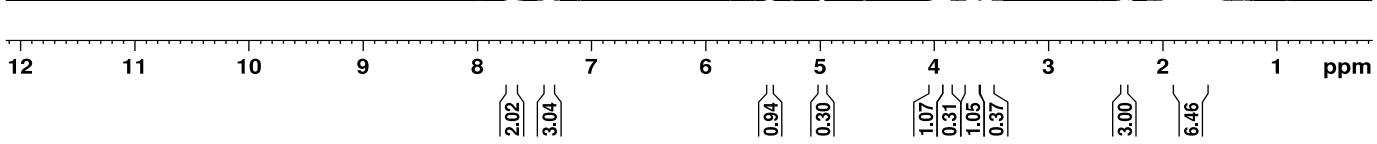

${ }^{13} \mathrm{C}\left\{{ }^{1} \mathrm{H}\right\}$ NMR (75.5 MHz) Spectrum of a 1:3 Z/E mixture of 7a in $\mathrm{CDCl}_{3}$

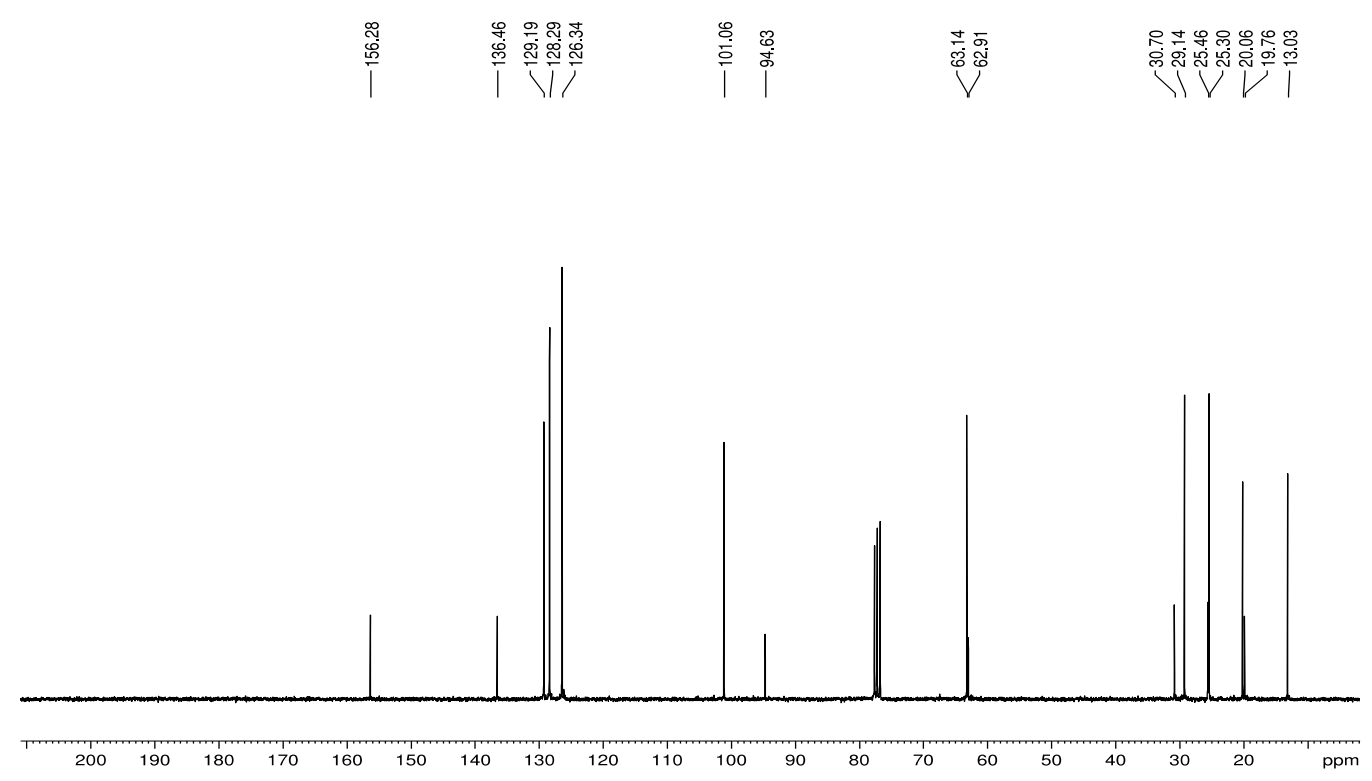


$\underline{{ }^{1} \mathrm{H} \mathrm{NMR}(300 \mathrm{MHz}) \text { Spectrum of } 7 \mathbf{a}\left(\boldsymbol{E} \text { isomer) in } \mathrm{CDCl}_{3}\right.}$<smiles>C[N+](=O)/C(=N\OC1CCCCO1)c1ccccc1</smiles>
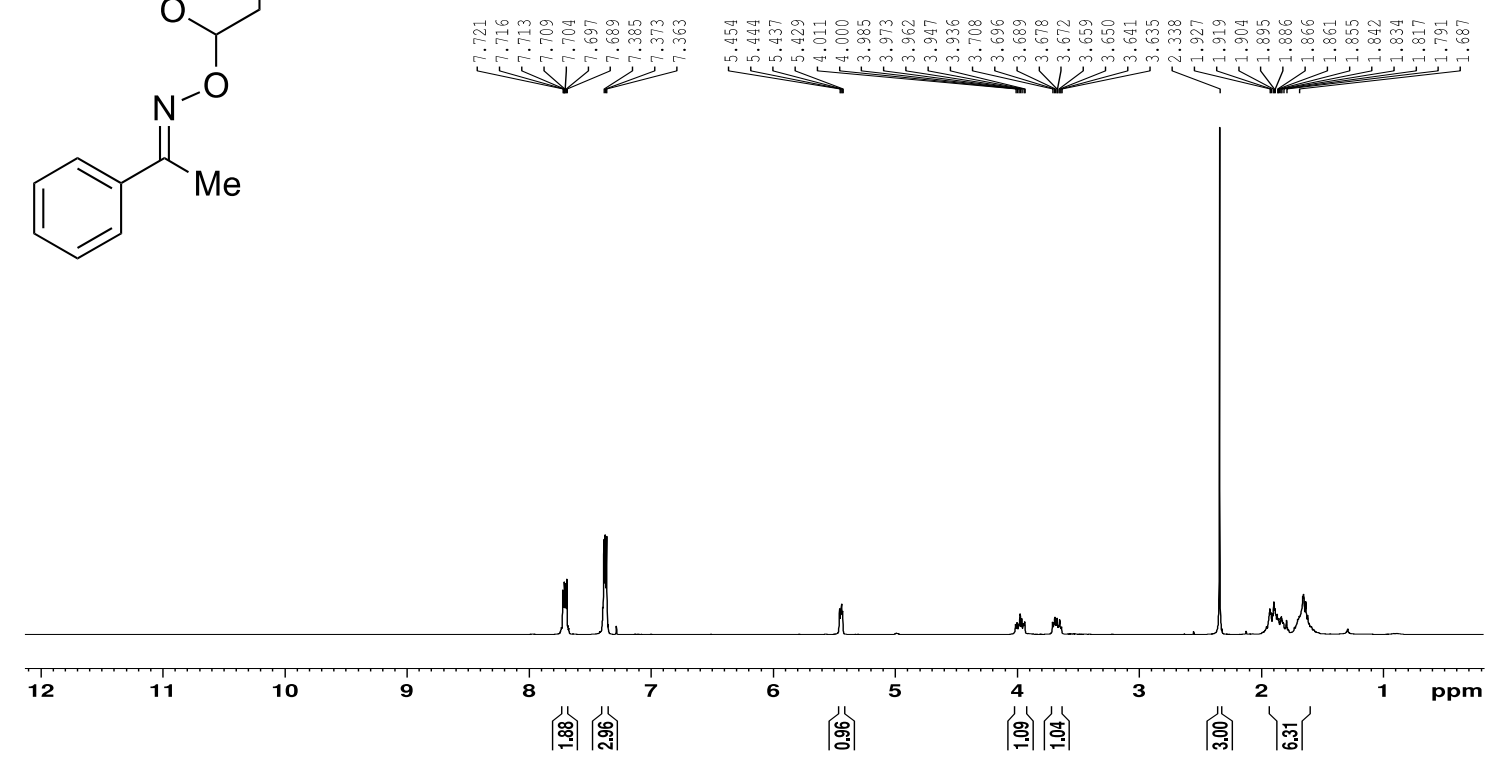

${ }^{13} \mathrm{C}\left\{{ }^{1} \mathrm{H}\right\}$ NMR (75.5 MHz) Spectrum of $7 \mathbf{a}\left(\boldsymbol{E}\right.$ isomer) in $\mathrm{CDCl}_{3}$

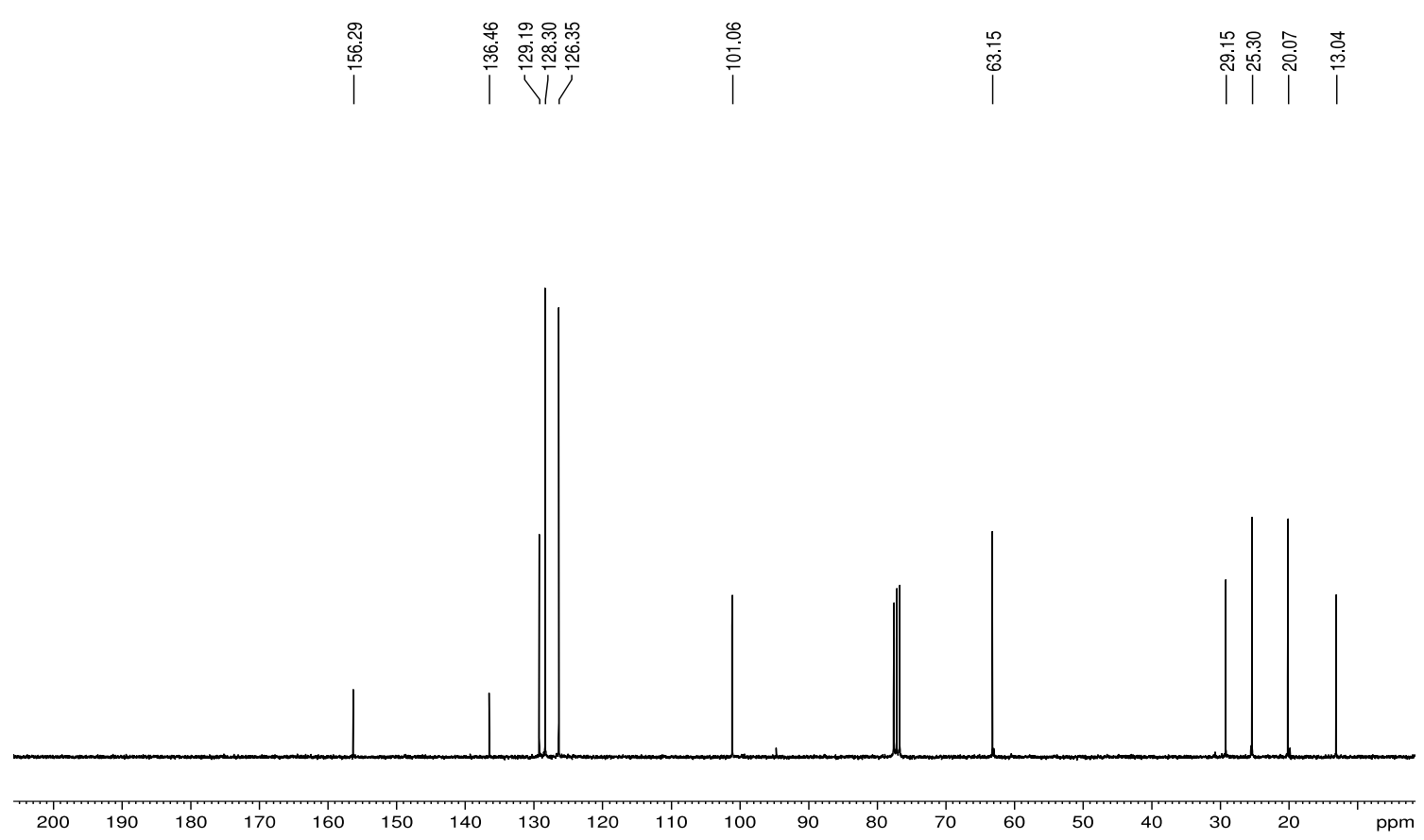




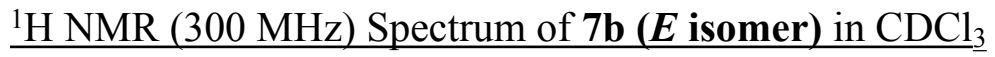

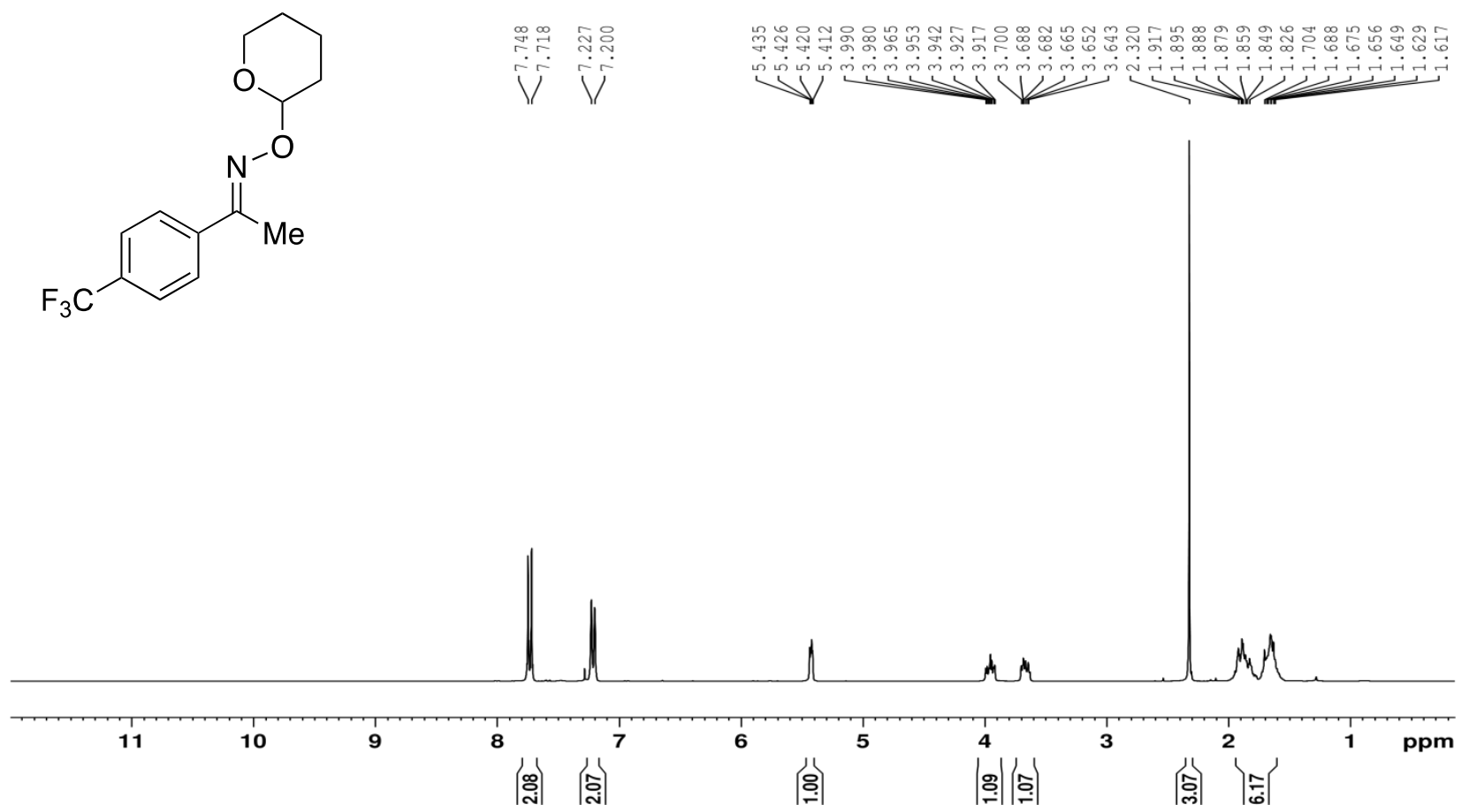

${ }^{13} \mathrm{C}\left\{{ }^{1} \mathrm{H}\right\}$ NMR (75.5 MHz) Spectrum of $7 \mathbf{b}\left(\boldsymbol{E}\right.$ isomer) in $\mathrm{CDCl}_{3}$

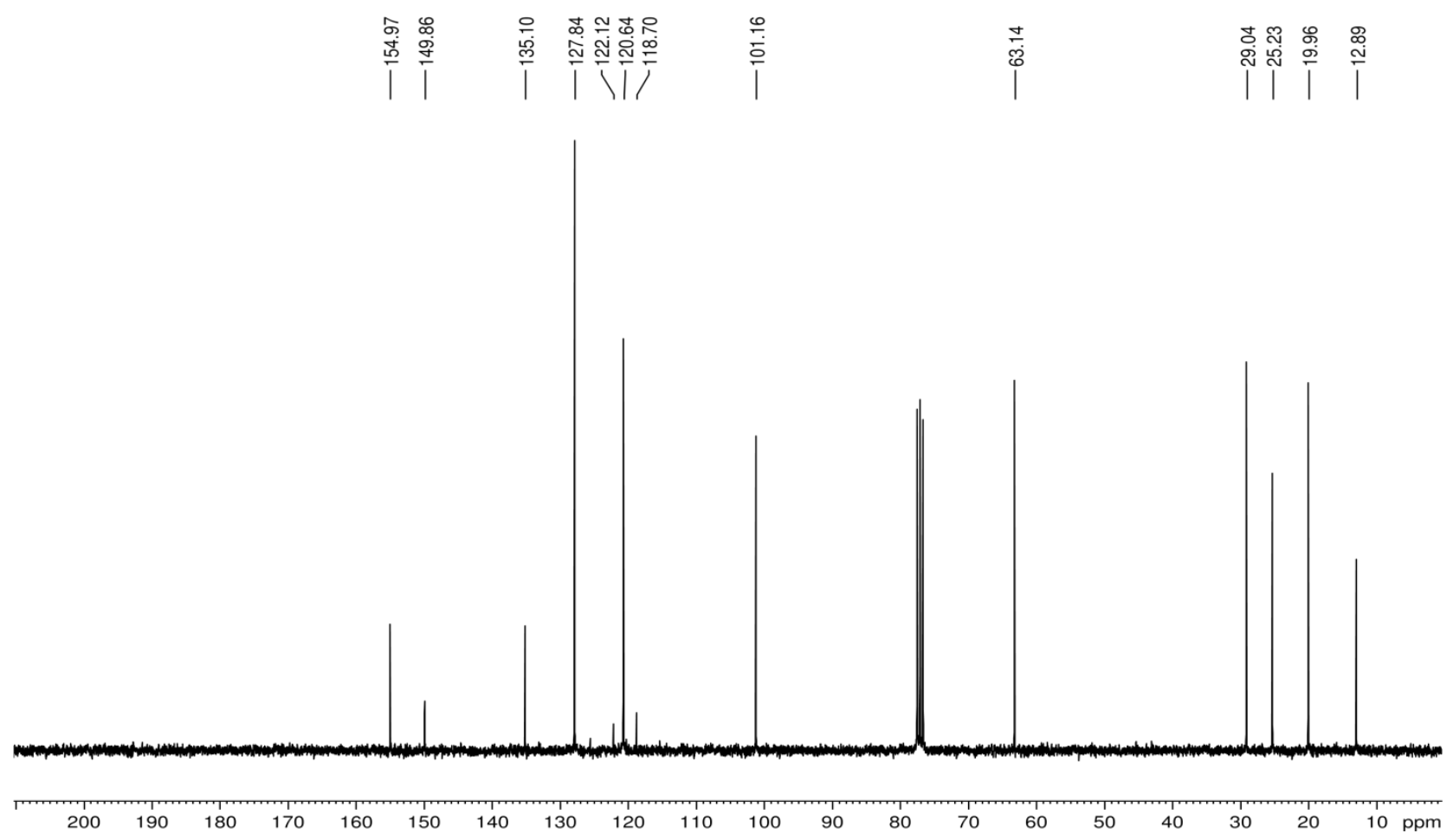


$\underline{{ }^{1} \mathrm{H} N M R}(300 \mathrm{MHz})$ Spectrum of $7 \mathbf{c}\left(\boldsymbol{E}\right.$ isomer) in $\mathrm{CDCl}_{3}$<smiles>C[N+](=O)/C(=N\OC1CCCCO1)c1ccc(OC(F)(F)F)cc1</smiles>
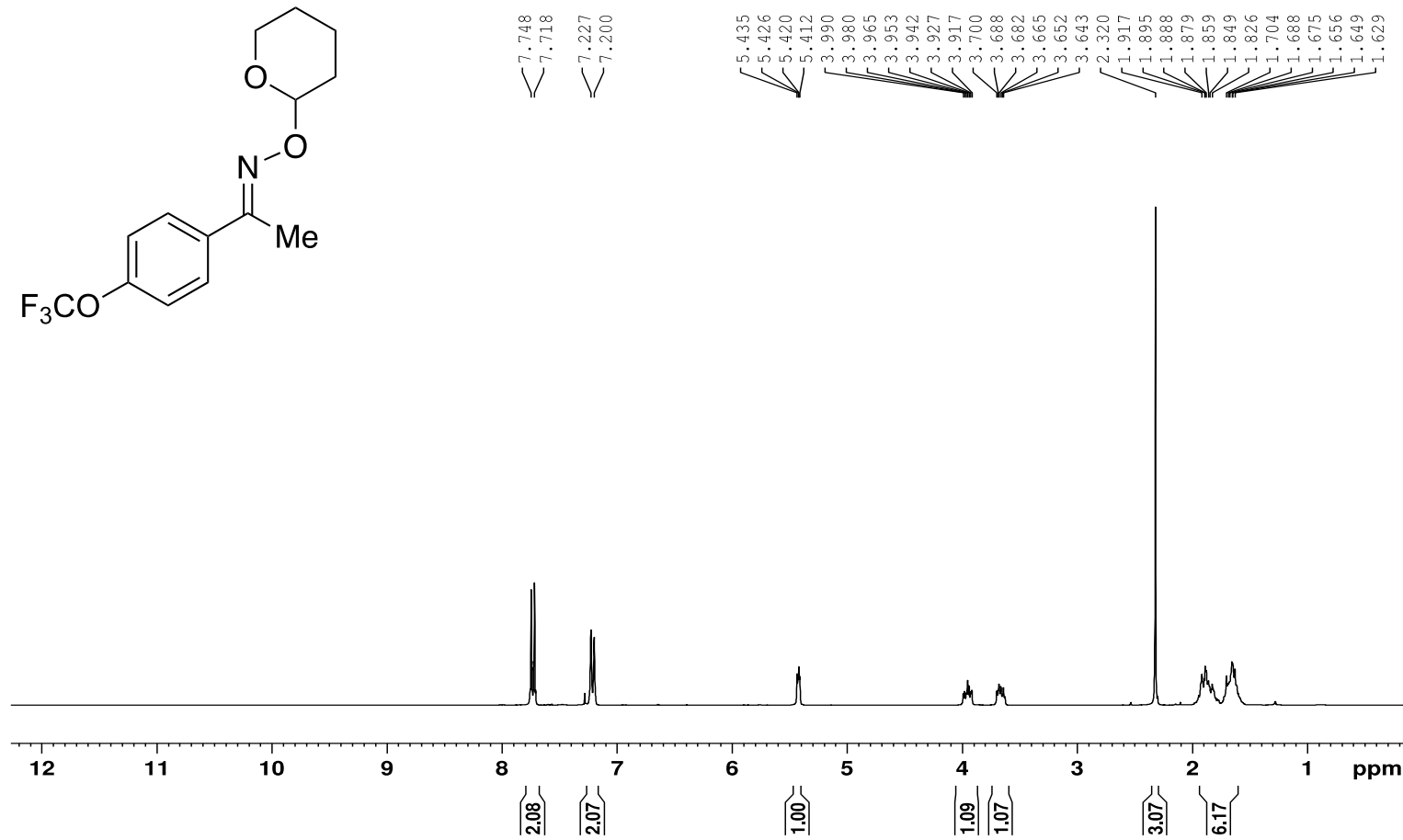

${ }^{13} \mathrm{C}\left\{{ }^{1} \mathrm{H}\right\}$ NMR (75.5 MHz) Spectrum of $7 \mathbf{c}\left(\boldsymbol{E}\right.$ isomer) in $\mathrm{CDCl}_{3}$

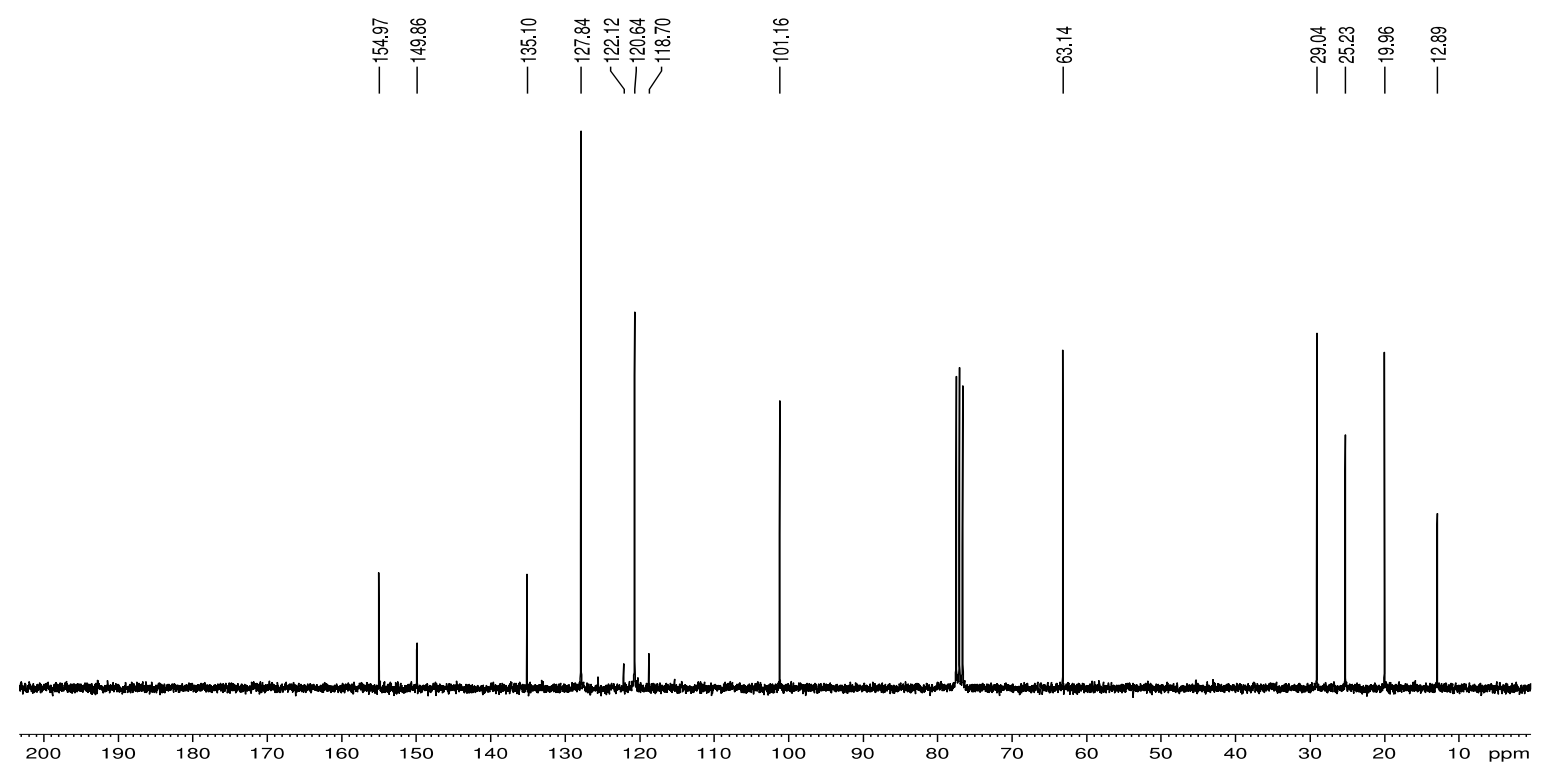


$\underline{{ }^{1} \mathrm{H} N M R}(300 \mathrm{MHz})$ Spectrum of $\mathbf{7 d}\left(\boldsymbol{E}\right.$ isomer) in $\mathrm{CDCl}_{3}$

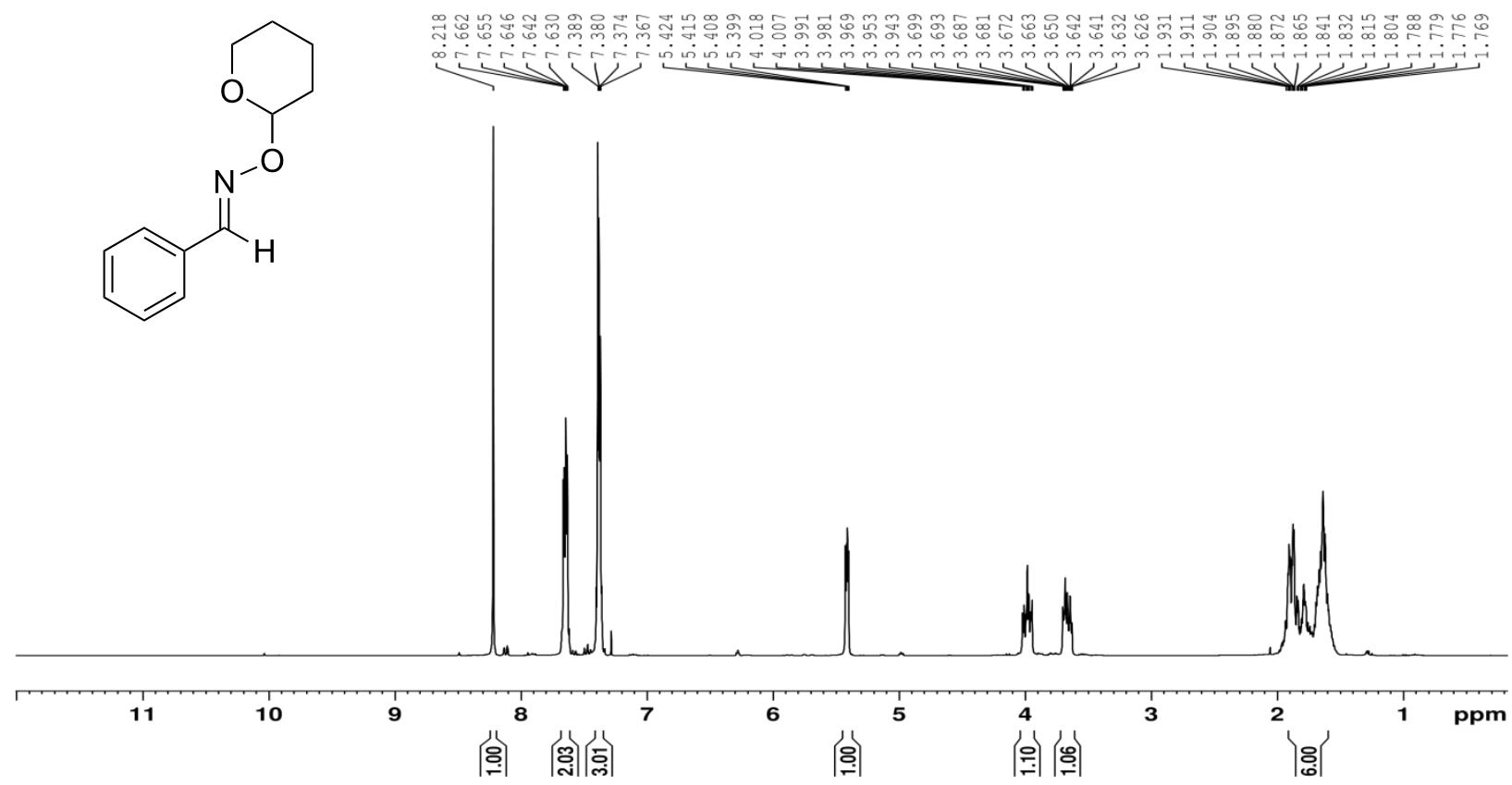

${ }^{13} \mathrm{C}\left\{{ }^{1} \mathrm{H}\right\}$ NMR (75.5 MHz) Spectrum of $7 \mathbf{d}\left(\boldsymbol{E}\right.$ isomer) in $\mathrm{CDCl}_{3}$

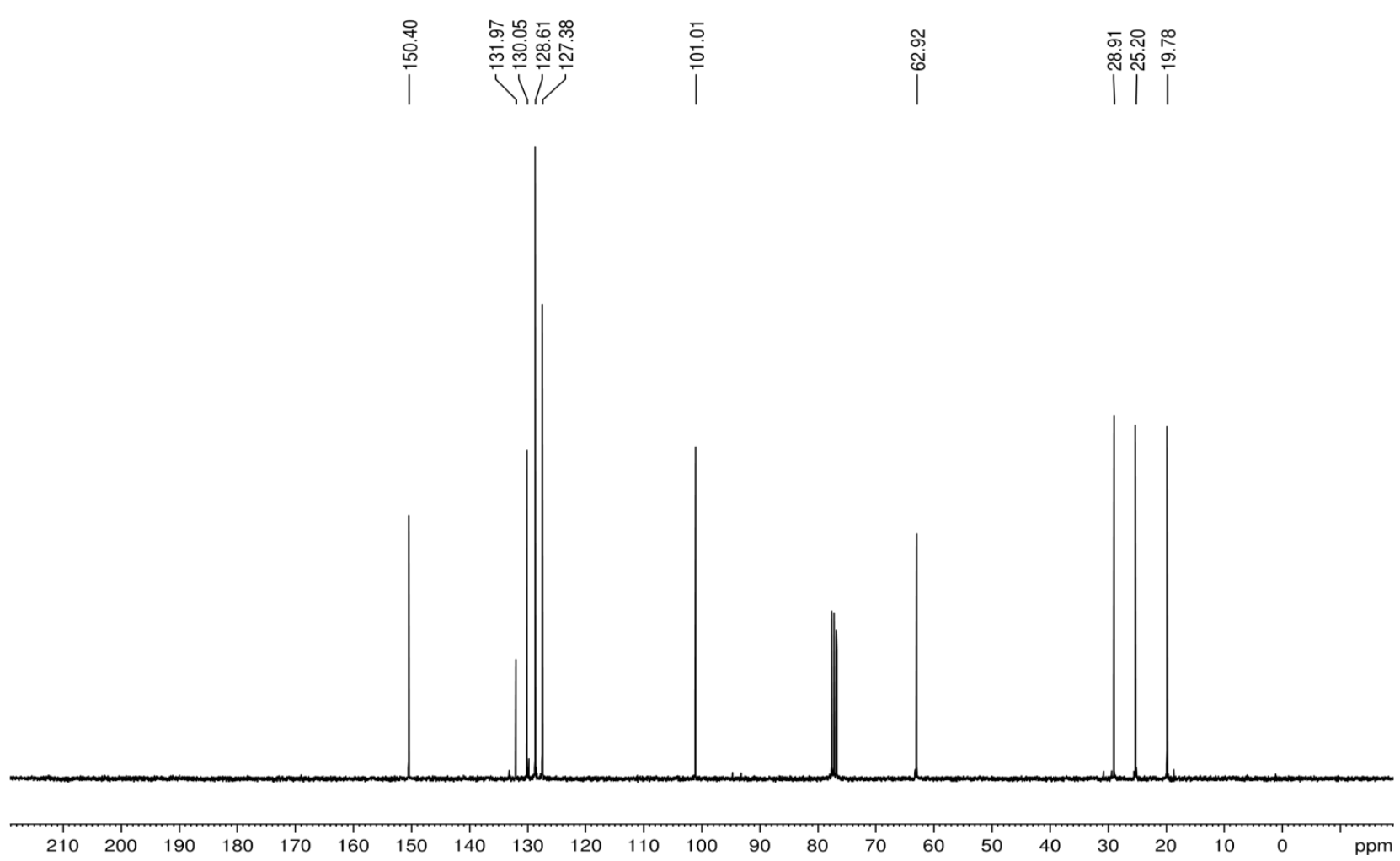


$\underline{{ }^{1} \mathrm{H} \mathrm{NMR}(300 \mathrm{MHz}) \text { Spectrum of } 7 \mathbf{d}\left(\boldsymbol{Z} \text { isomer) in } \mathrm{CDCl}_{3}\right.}$

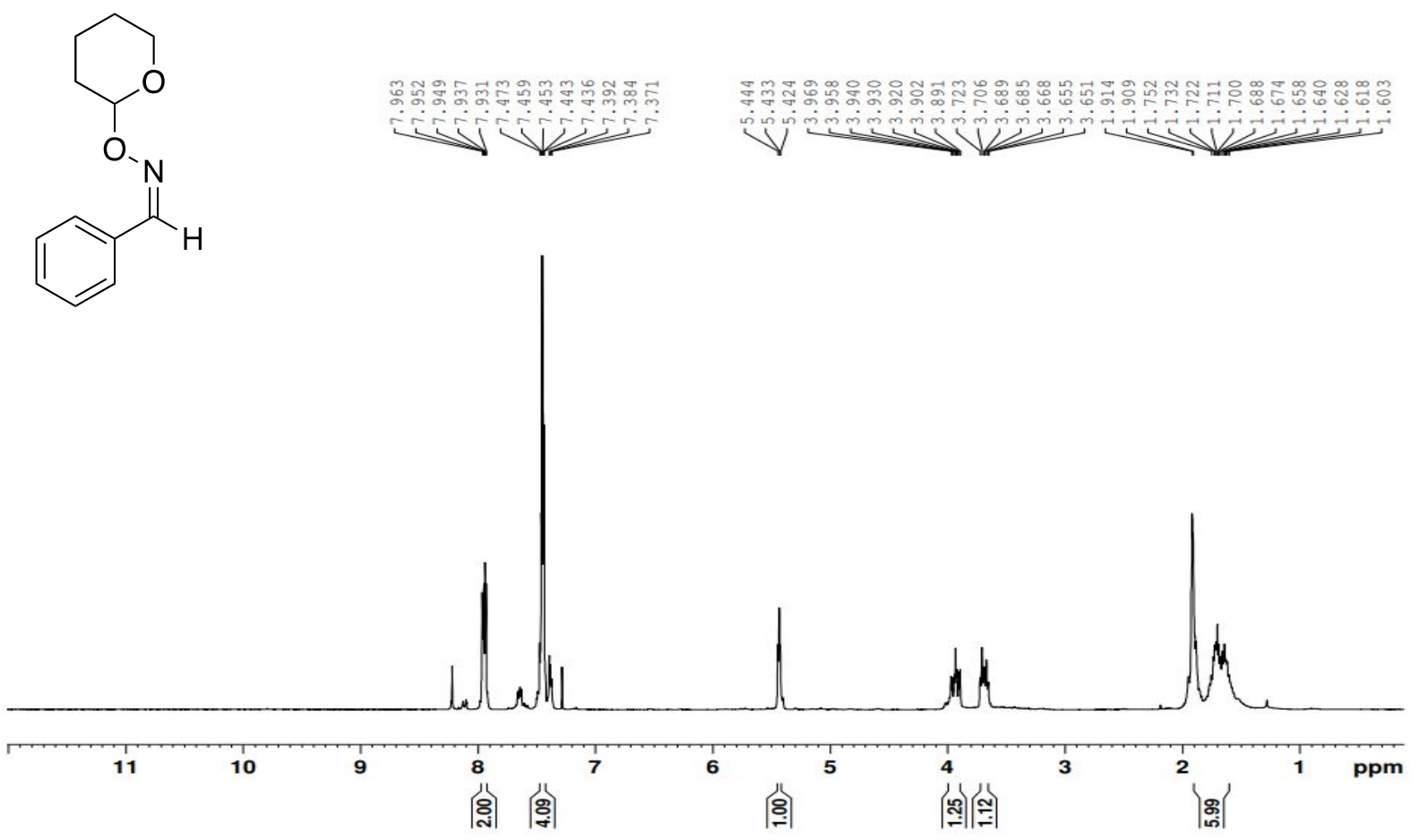

${ }^{13} \mathrm{C}\left\{{ }^{1} \mathrm{H}\right\}$ NMR (75.5 MHz) Spectrum of $7 \mathbf{d}\left(\boldsymbol{Z}\right.$ isomer) in $\mathrm{CDCl}_{3}$

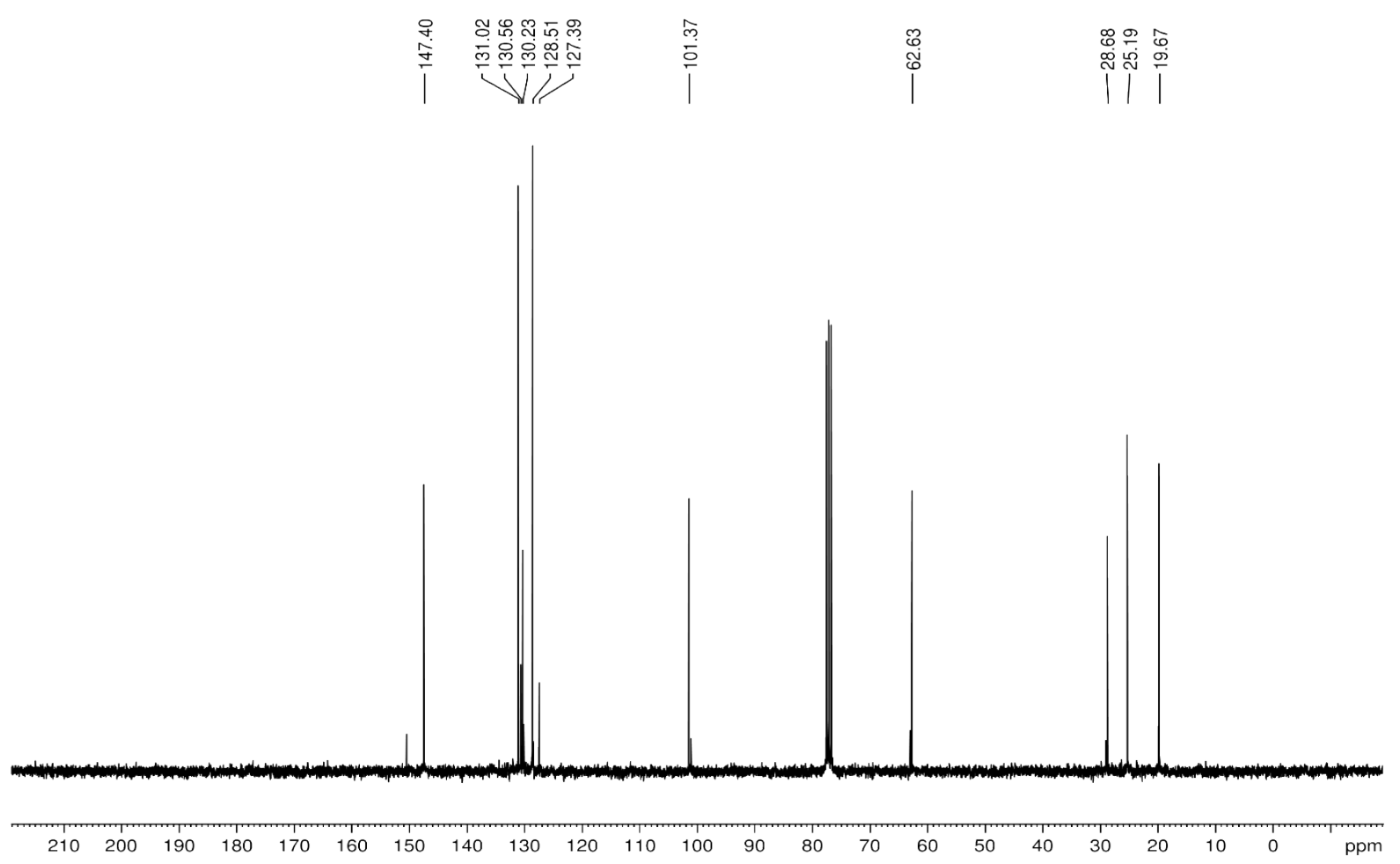


$\underline{{ }^{1} \mathrm{H} \text { NMR }(300 \mathrm{MHz}) \text { Spectrum of } 7 \mathrm{e}\left(\boldsymbol{E} \text { isomer) in } \mathrm{CDCl}_{3}\right.}$<smiles>Cc1ccc(/C=N/OC2CCCCO2)cc1</smiles>

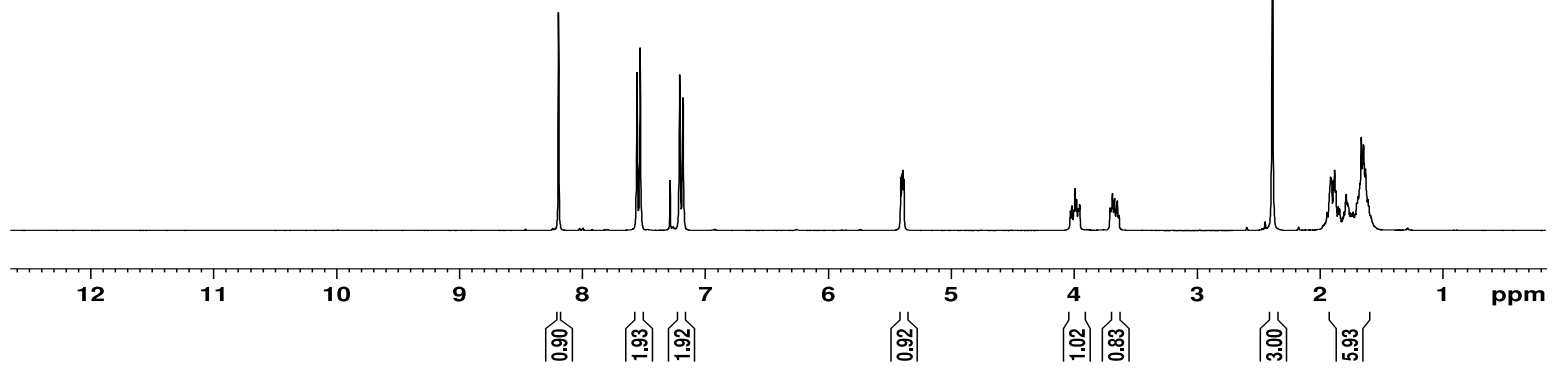

${ }^{13} \mathrm{C}\left\{{ }^{1} \mathrm{H}\right\}$ NMR (75.5 MHz) Spectrum of $7 \mathbf{e}\left(\boldsymbol{E}\right.$ isomer) in $\mathrm{CDCl}_{3}$

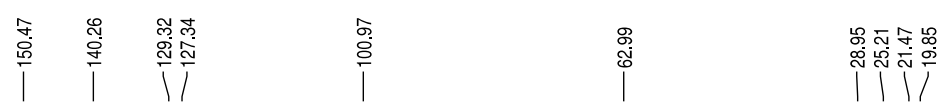

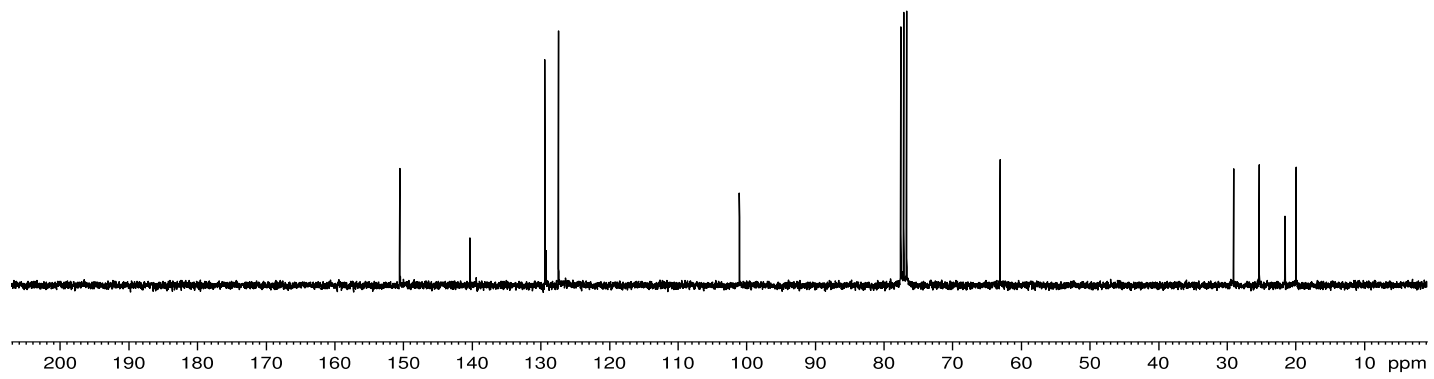


$\underline{{ }^{1} \mathrm{H} \text { NMR }(300 \mathrm{MHz}) \text { Spectrum of } 7 \mathrm{e}\left(\boldsymbol{Z} \text { isomer) in } \mathrm{CDCl}_{3}\right.}$<smiles>Cc1ccc(/C=N/OC2CCCCO2)cc1</smiles>
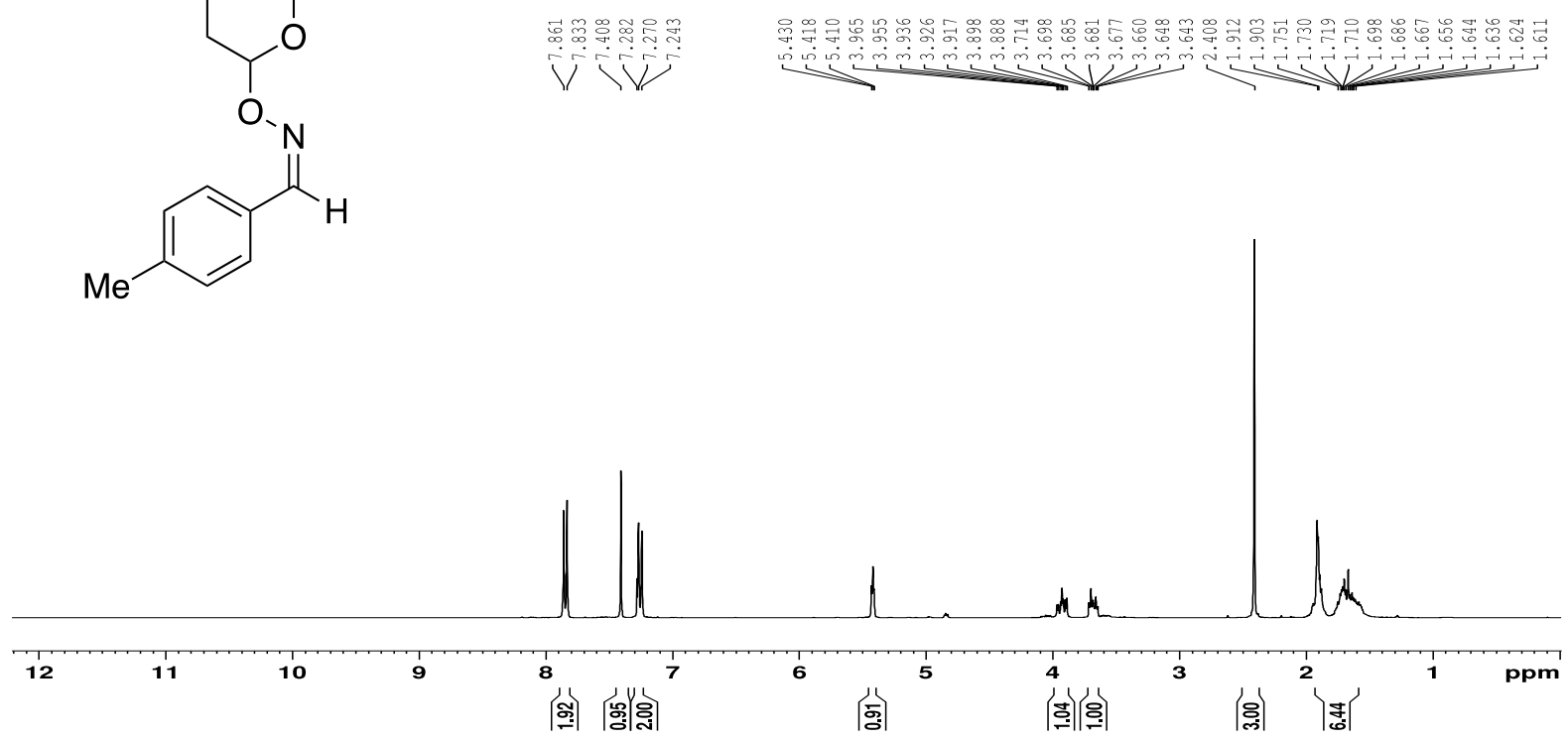

${ }^{13} \mathrm{C}\left\{{ }^{1} \mathrm{H}\right\}$ NMR (75.5 MHz) Spectrum of $7 \mathbf{e}\left(\boldsymbol{Z}\right.$ isomer) in $\mathrm{CDCl}_{3}$
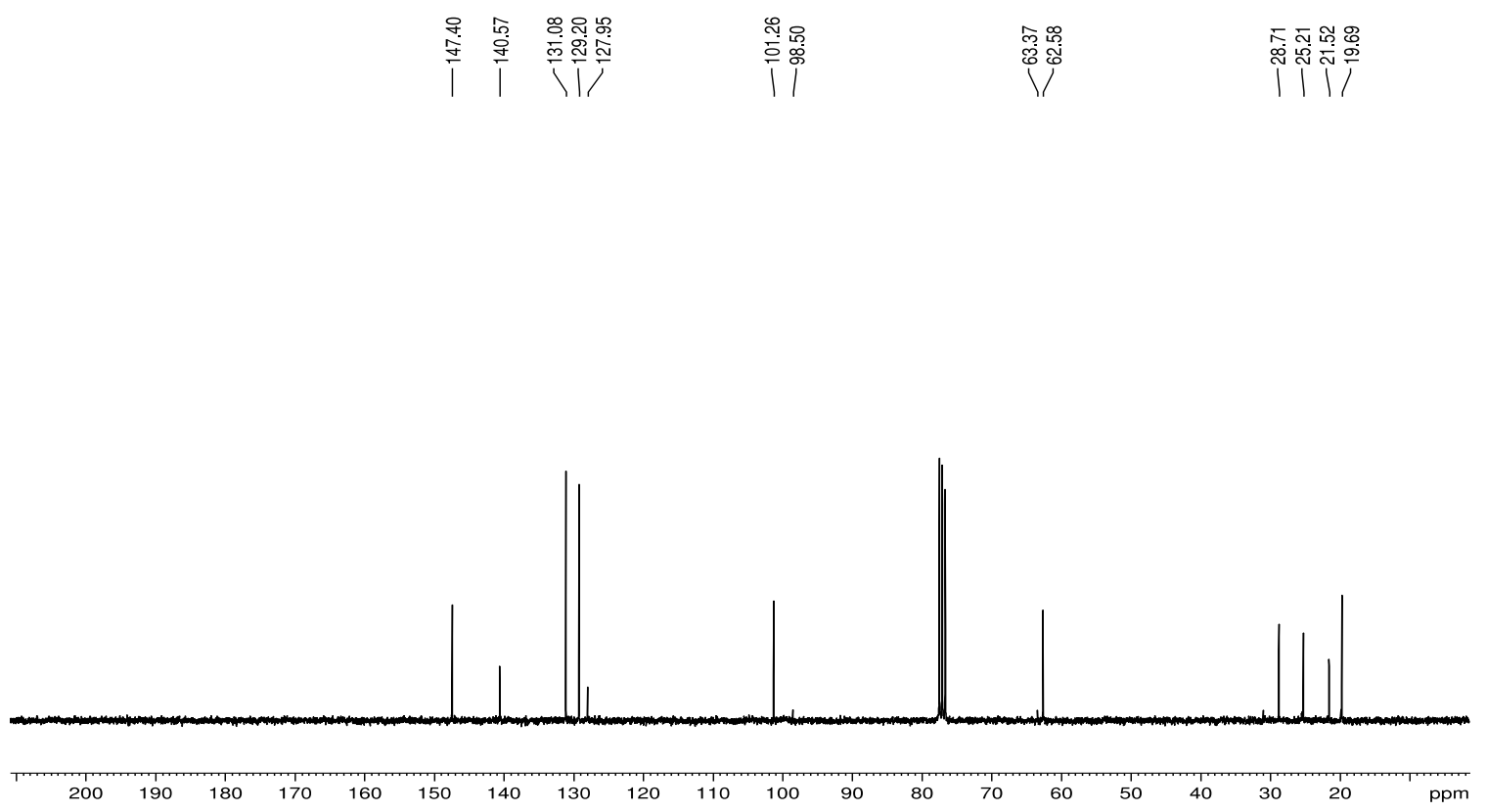
$\underline{{ }^{1} \mathrm{H} \mathrm{NMR}(300 \mathrm{MHz}) \text { Spectrum of } 7 \mathbf{f}\left(\boldsymbol{E} \text { isomer) in } \mathrm{CDCl}_{3}\right.}$

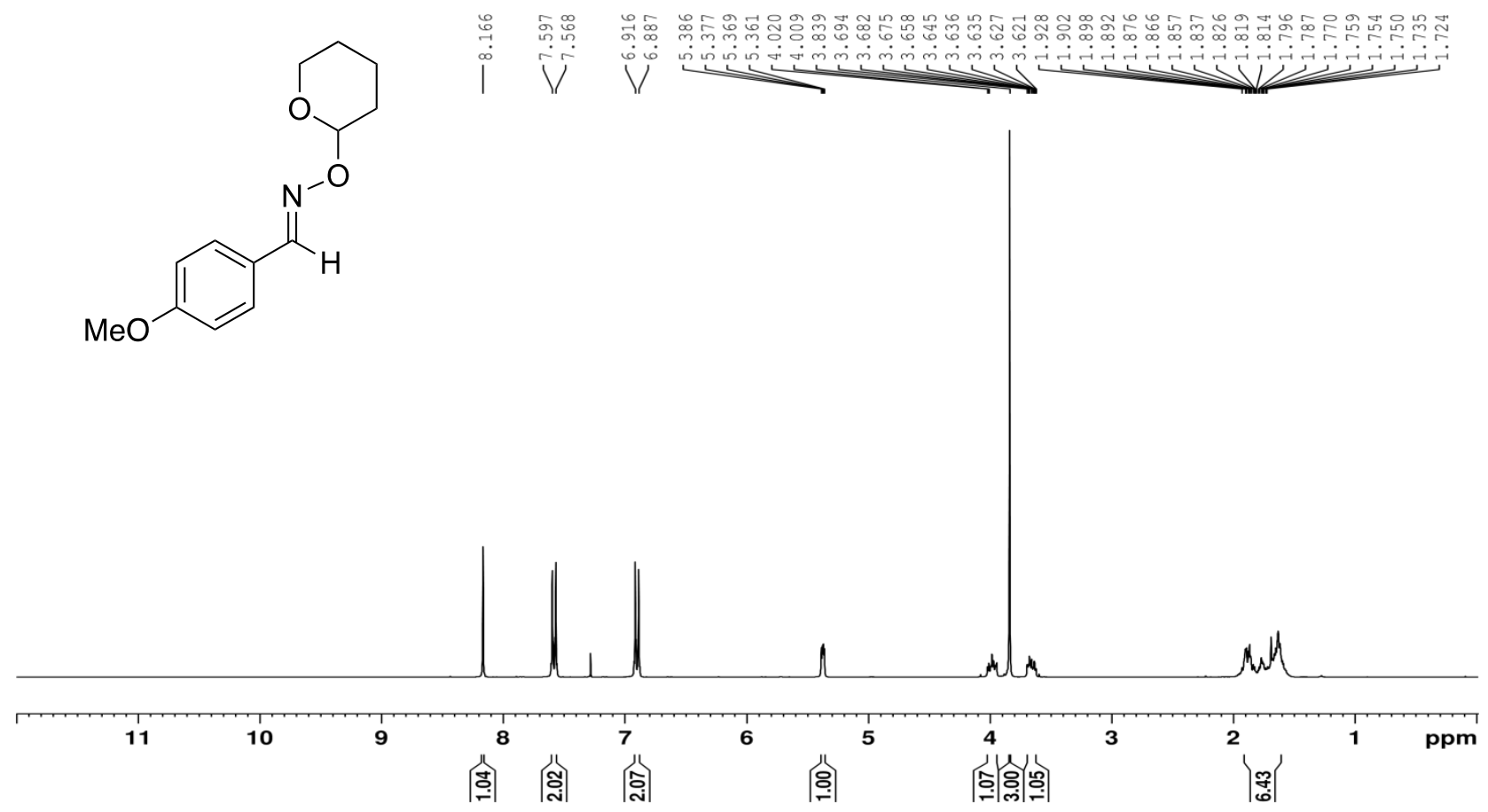

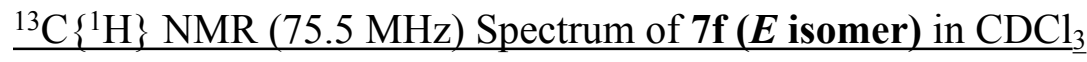

$$
\text { li }
$$
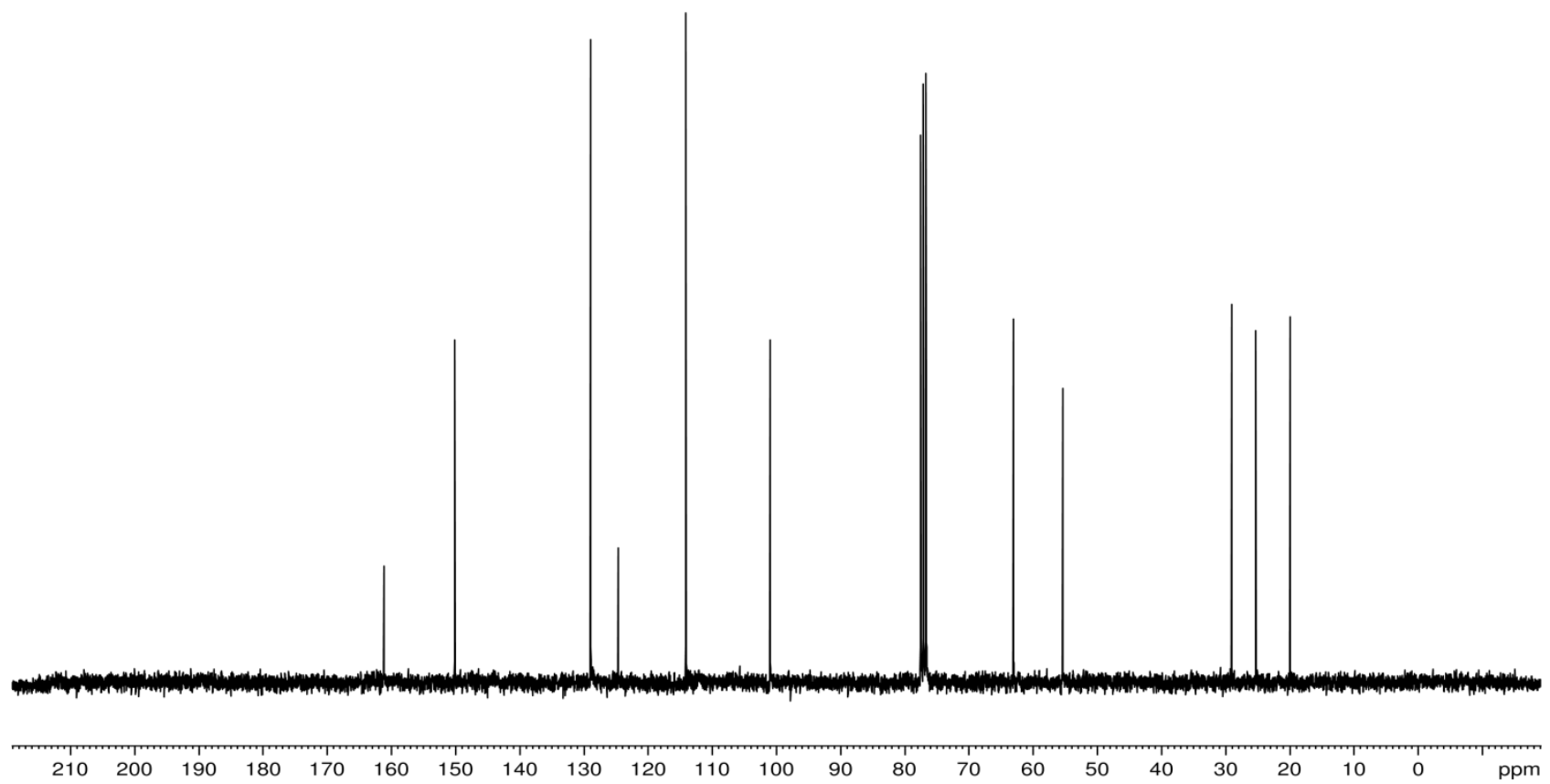
$\underline{{ }^{1} \mathrm{H} \mathrm{NMR}(300 \mathrm{MHz}) \text { Spectrum of } 7 \mathbf{f}\left(\boldsymbol{Z} \text { isomer) in } \mathrm{CDCl}_{3}\right.}$

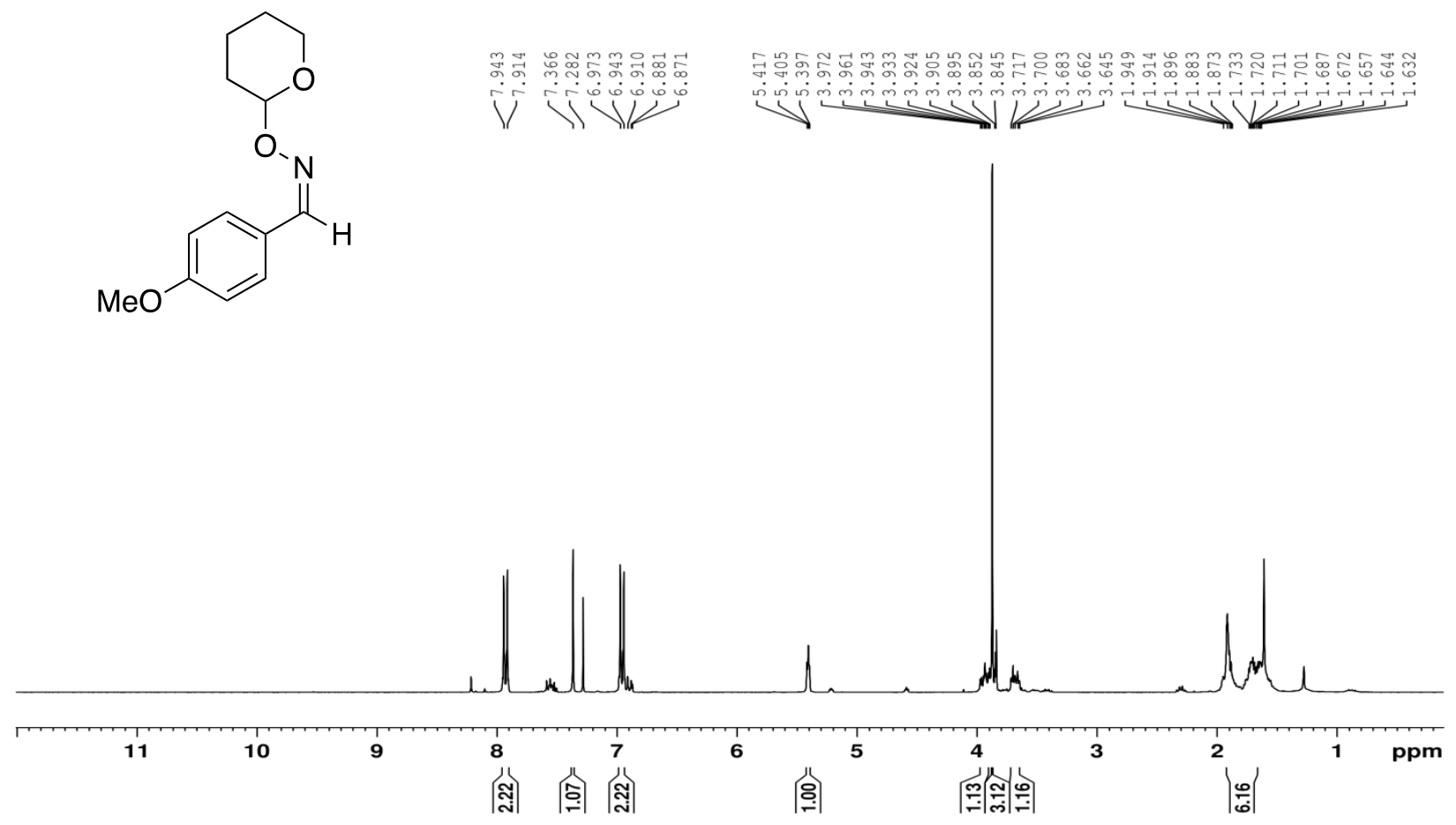

${ }^{13} \mathrm{C}\left\{{ }^{1} \mathrm{H}\right\}$ NMR (75.5 MHz) Spectrum of $7 \mathbf{f}\left(\boldsymbol{Z}\right.$ isomer) in $\mathrm{CDCl}_{3}$

$$
\text { | }
$$

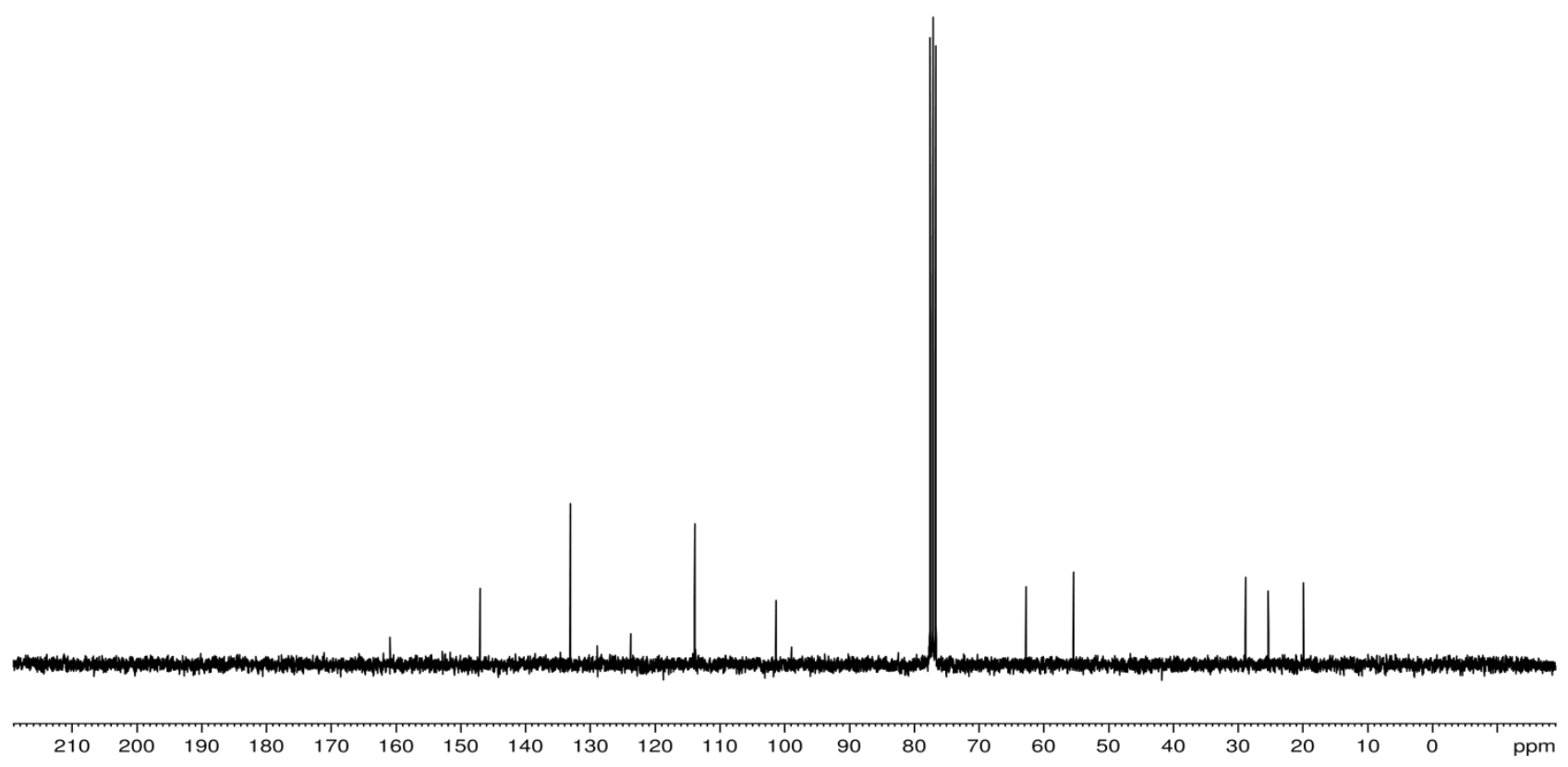




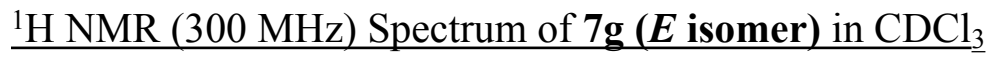

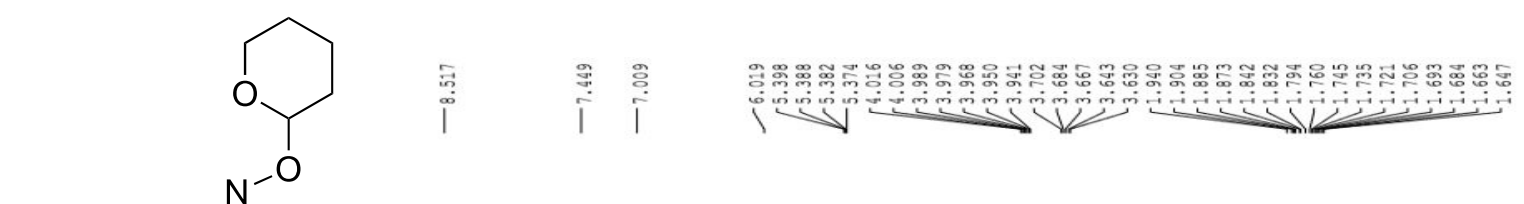

${ }^{13} \mathrm{C}\left\{{ }^{1} \mathrm{H}\right\}$ NMR (75.5 MHz) Spectrum of $7 \mathbf{g}\left(\boldsymbol{E}\right.$ isomer) in $\mathrm{CDCl}_{3}$

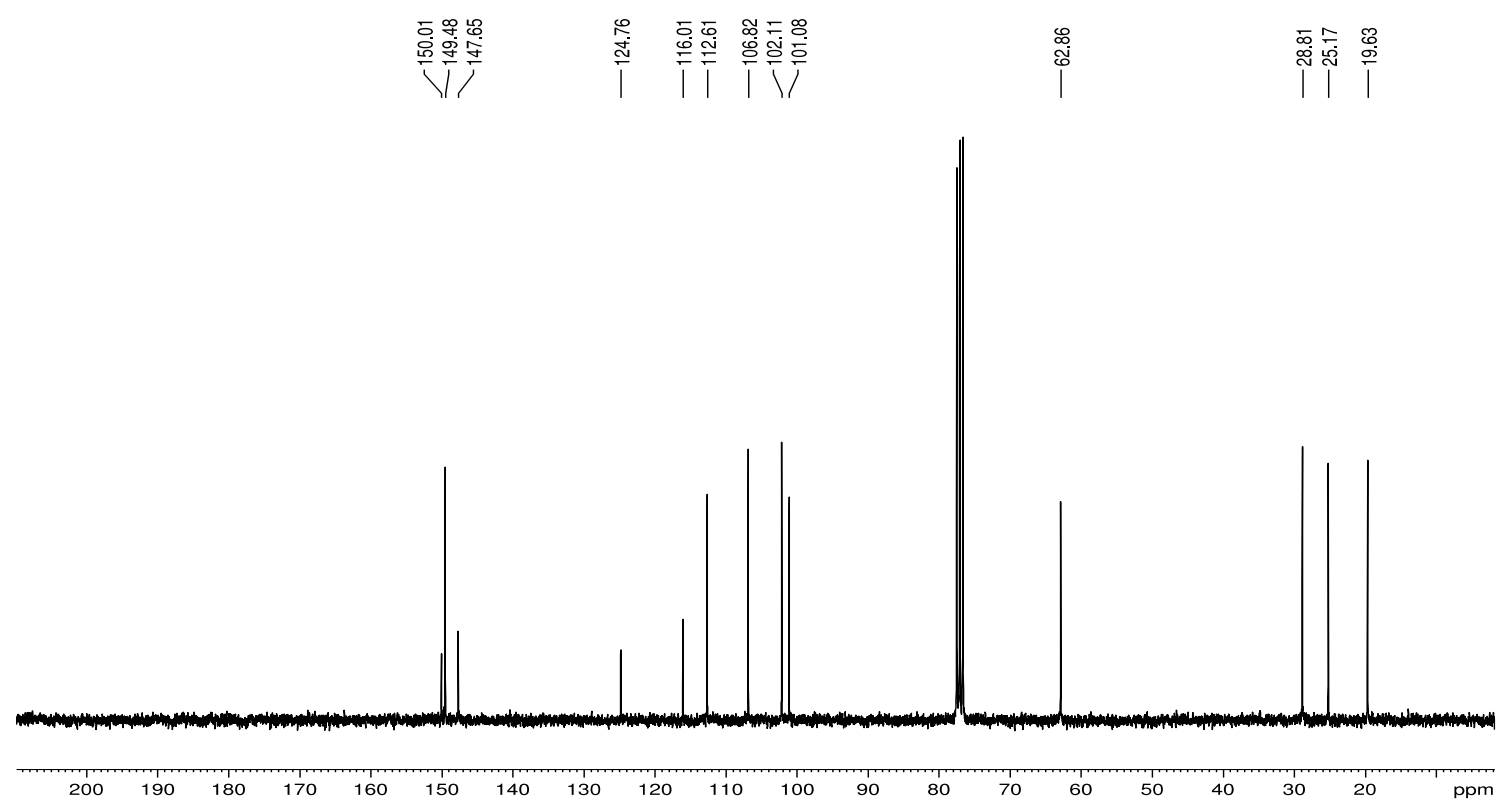


$\underline{{ }^{1} \mathrm{H} N M R}(300 \mathrm{MHz})$ Spectrum of $7 \mathbf{h}\left(\boldsymbol{E}\right.$ isomer) in $\mathrm{CDCl}_{3}$

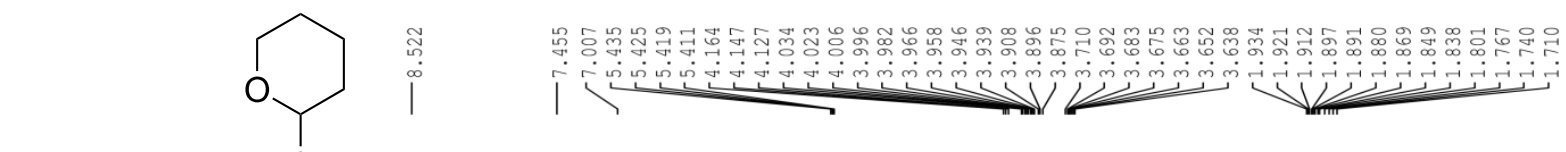<smiles>CO/N=C/c1cc(OC)c(OC)cc1Br</smiles>

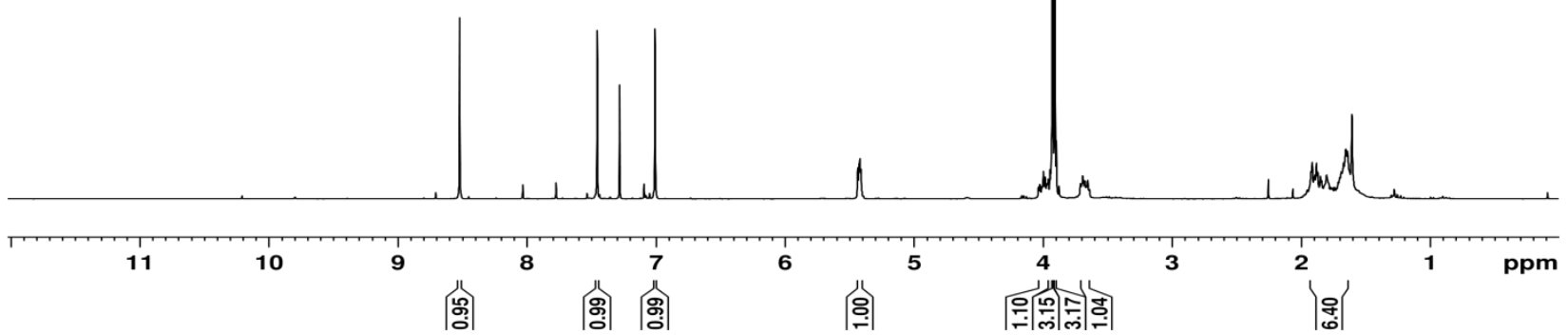

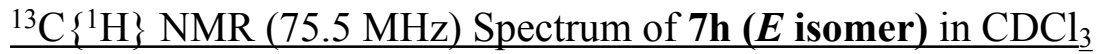

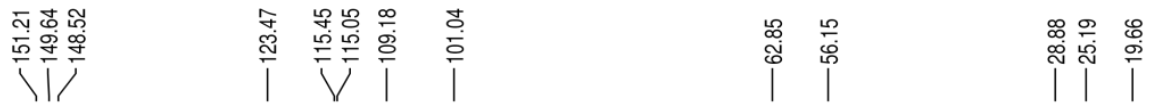




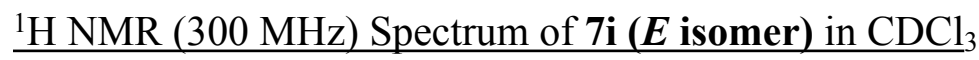

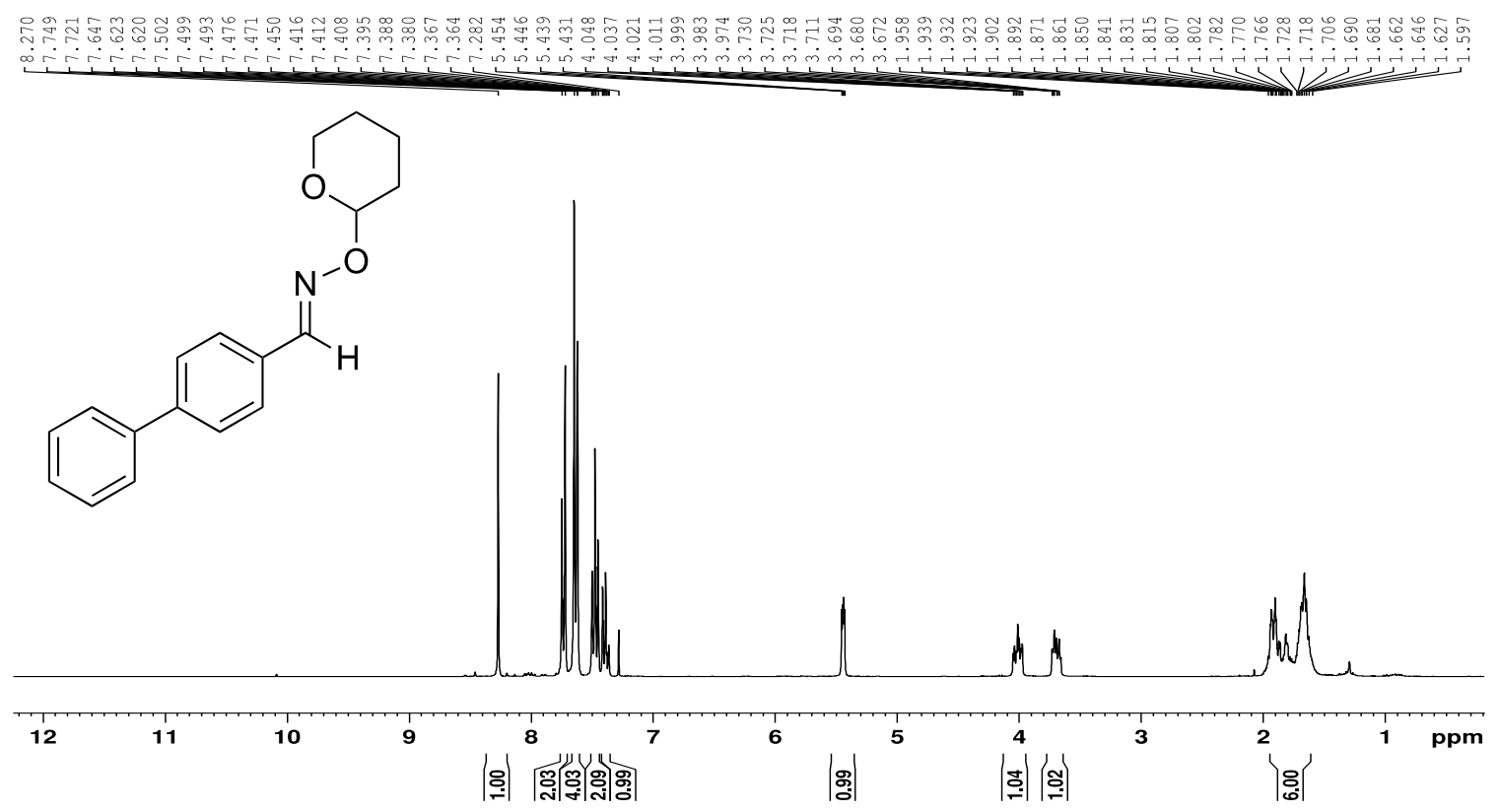

$\underline{{ }^{13} \mathrm{C}\left\{{ }^{1} \mathrm{H}\right\} \text { NMR (75.5 MHz) Spectrum of } 7 \mathbf{i}\left(\boldsymbol{E} \text { isomer) in } \mathrm{CDCl}_{3}\right.}$
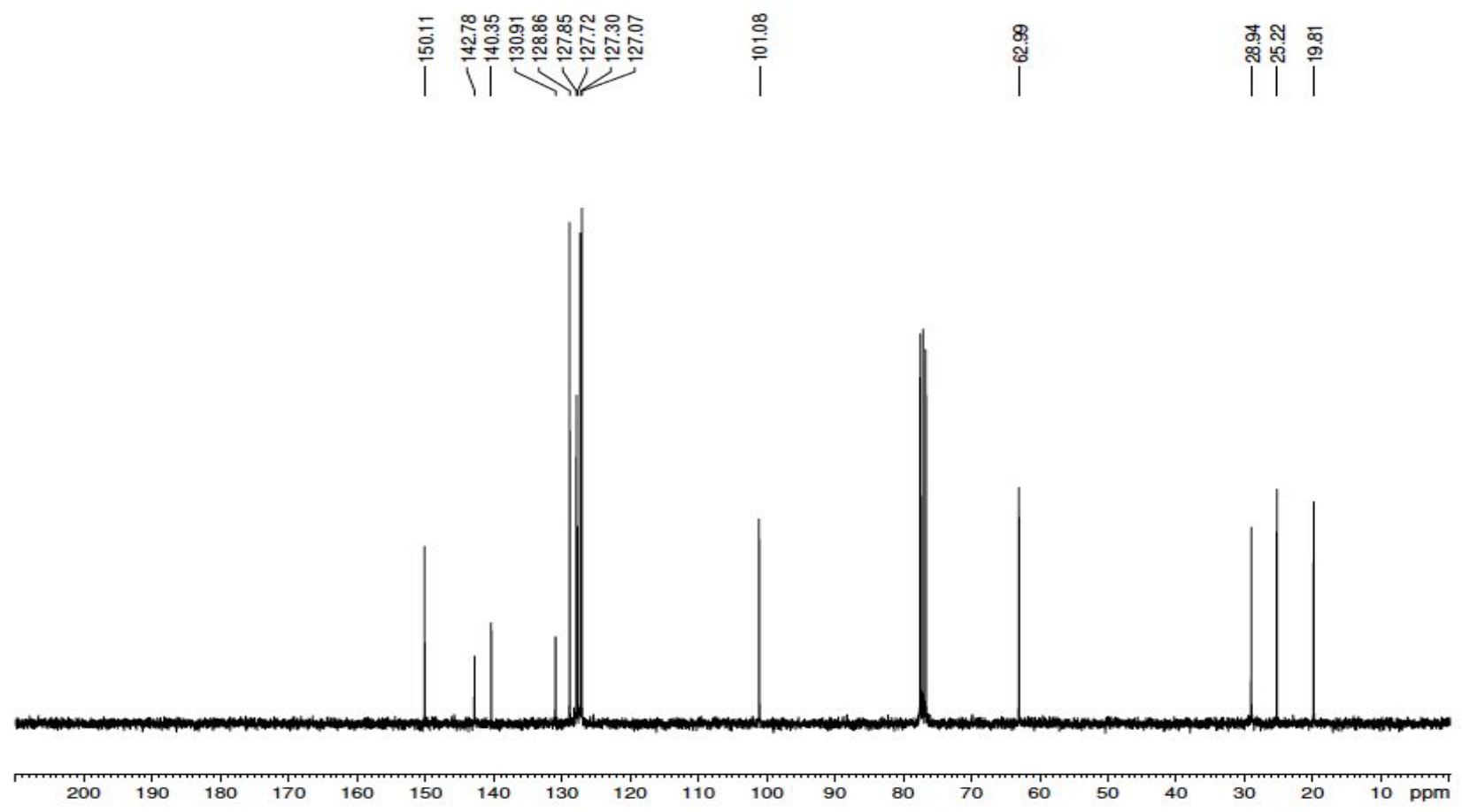
$\underline{{ }^{1} \mathrm{H} \mathrm{NMR}(300 \mathrm{MHz}) \text { Spectrum of } 7 \mathbf{j} \text { in } \mathrm{CDCl}_{3}}$

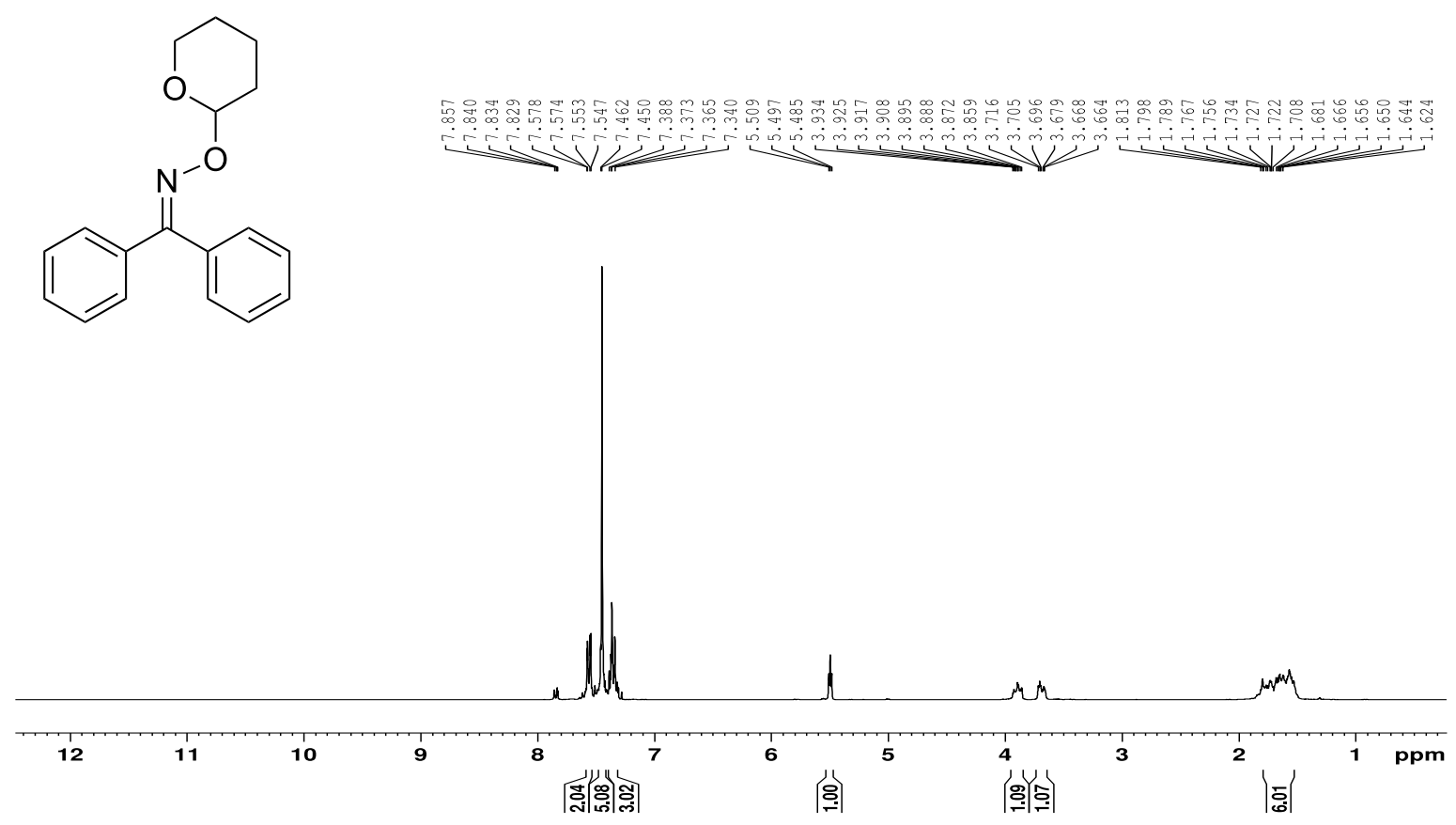

\section{${ }^{13} \mathrm{C}\left\{{ }^{1} \mathrm{H}\right\}(75.5 \mathrm{MHz})$ NMR Spectrum of $7 \mathbf{j}$ in $\mathrm{CDCl}_{3}$}

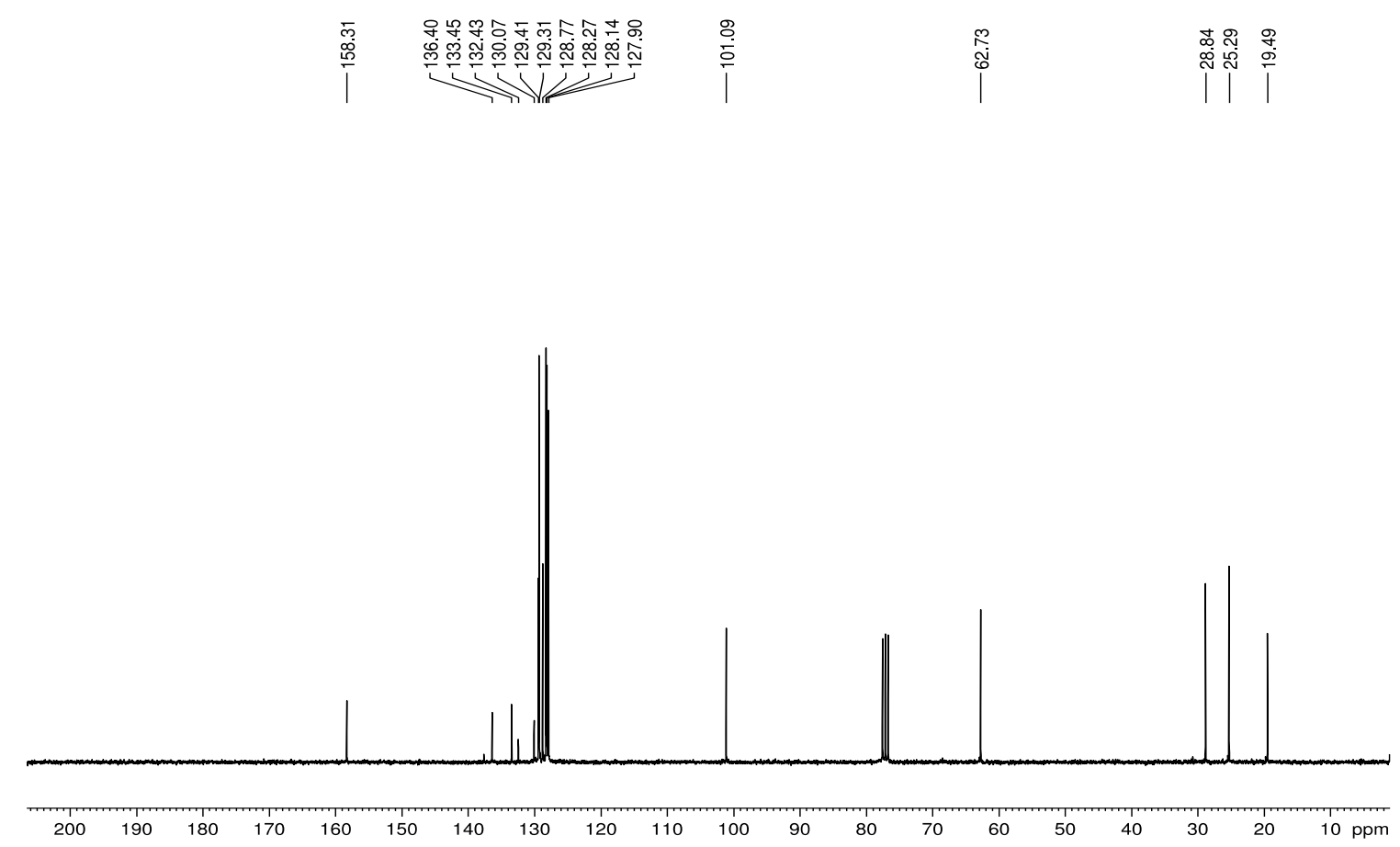


$\underline{{ }^{1} \mathrm{H} \mathrm{NMR}(300 \mathrm{MHz}) \text { Spectrum of } 7 \mathbf{k} \text { ( } \boldsymbol{E} \text { isomer) in } \mathrm{CDCl}_{3}}$

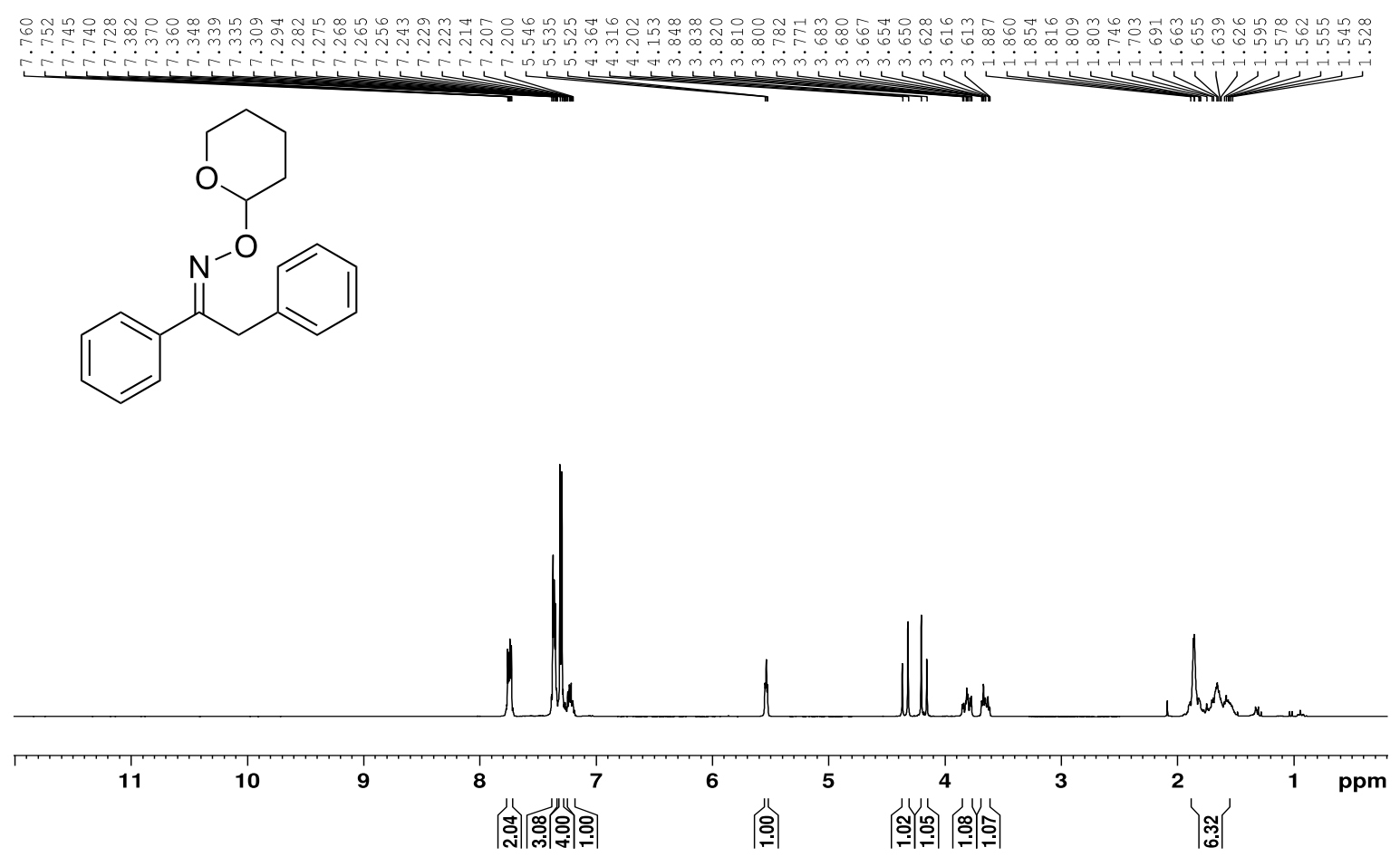

${ }^{13} \mathrm{C}\left\{{ }^{1} \mathrm{H}\right\}$ NMR (75.5 MHz) Spectrum of $7 \mathbf{k}\left(\boldsymbol{E}\right.$ isomer) in $\mathrm{CDCl}_{3}$

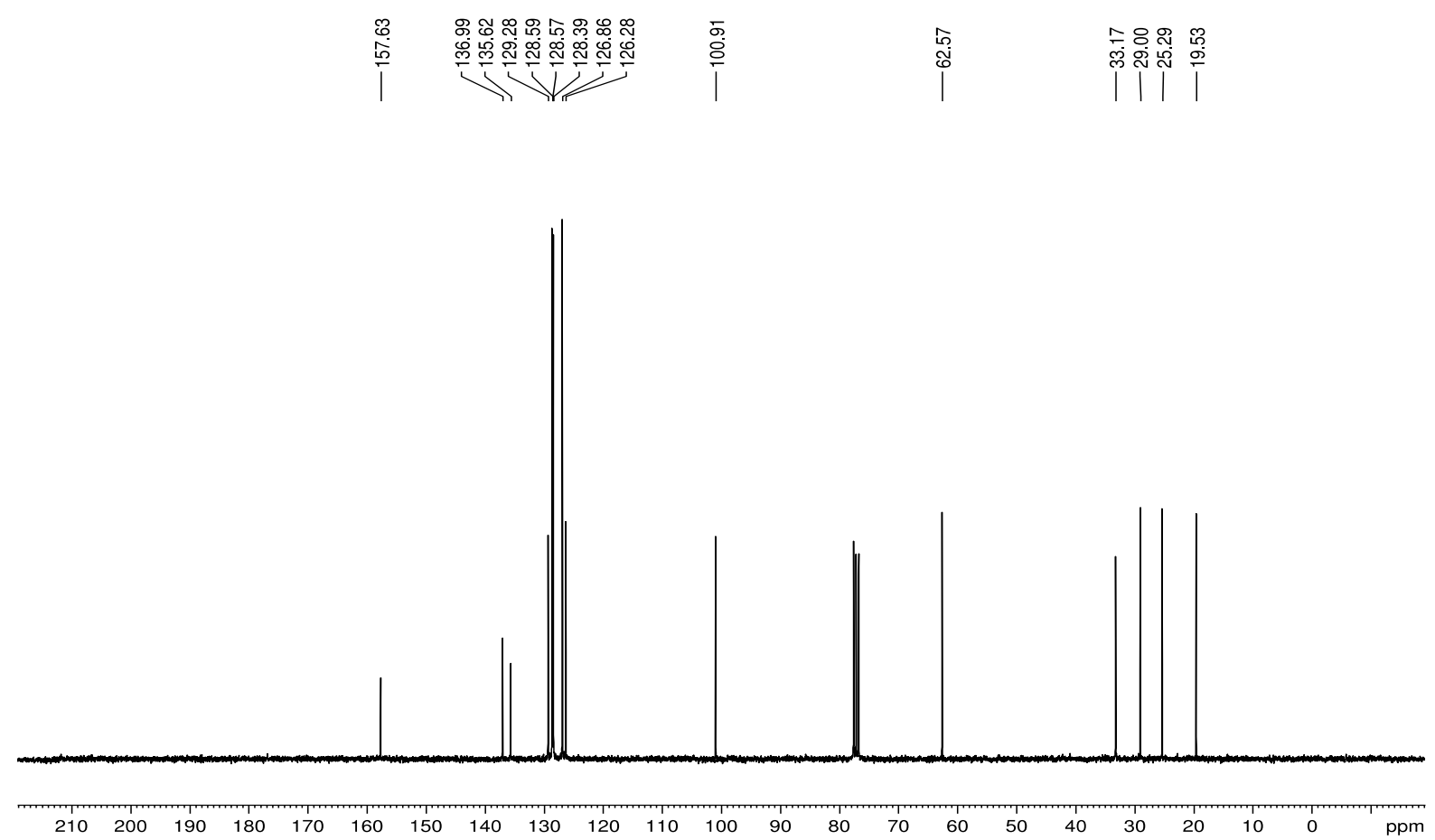


$\underline{{ }^{1} \mathrm{H} \text { NMR (300 MHz) Spectrum of a 1:6 Z/E mixture of } 71 \text { in } \mathrm{CDCl}_{3}}$

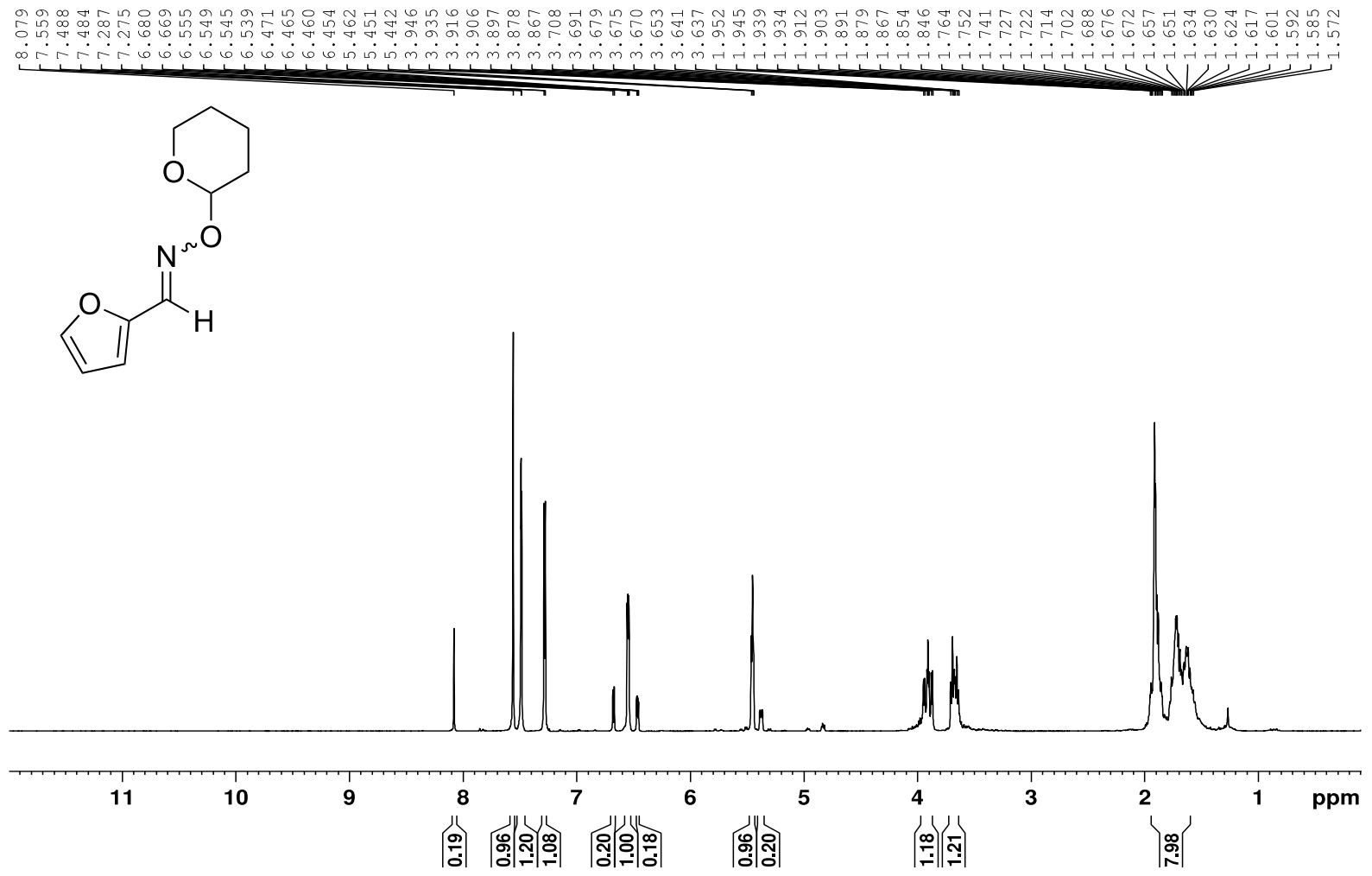

${ }^{13} \mathrm{C}\left\{{ }^{1} \mathrm{H}\right\}$ NMR (75.5 MHz) Spectrum of a 1:6 Z/E mixture of $7 \mathbf{l}$ in $\mathrm{CDCl}_{3}$

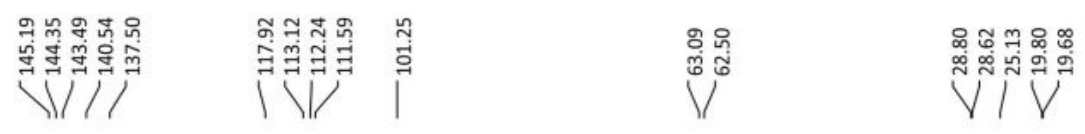

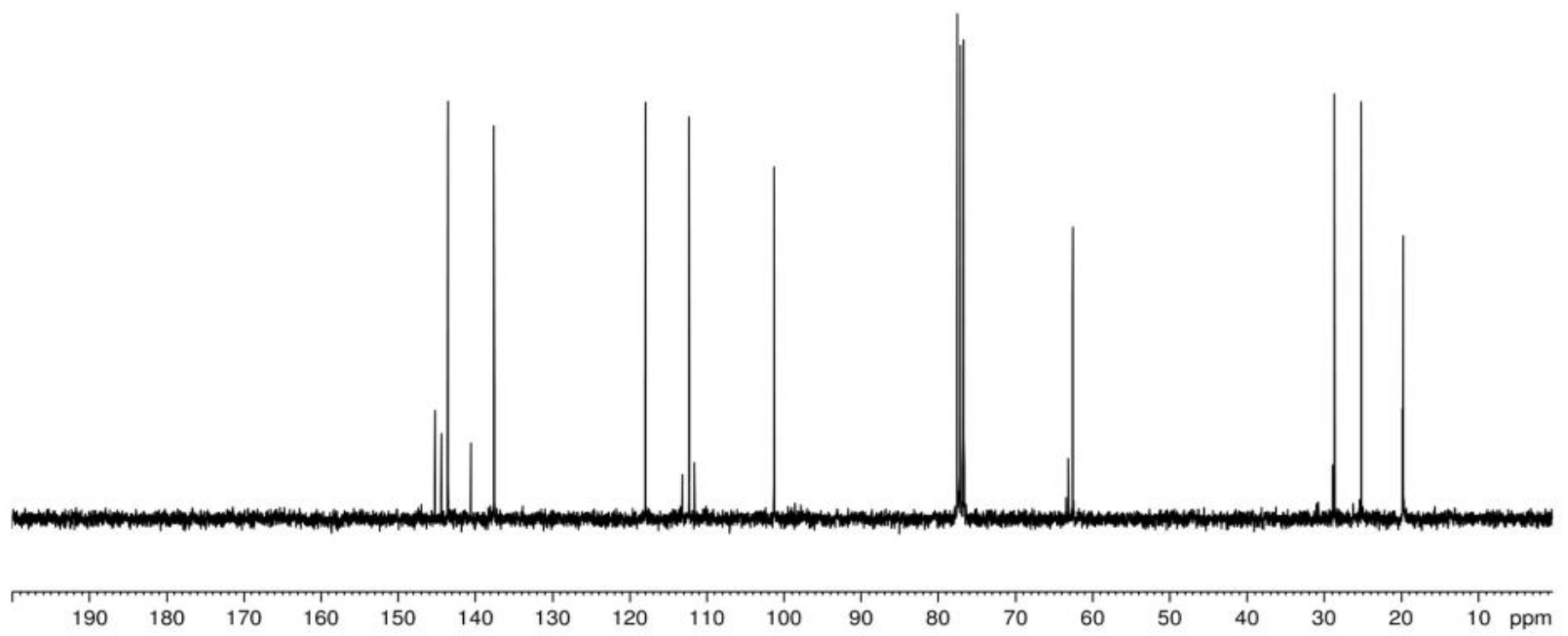




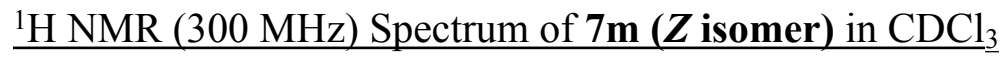

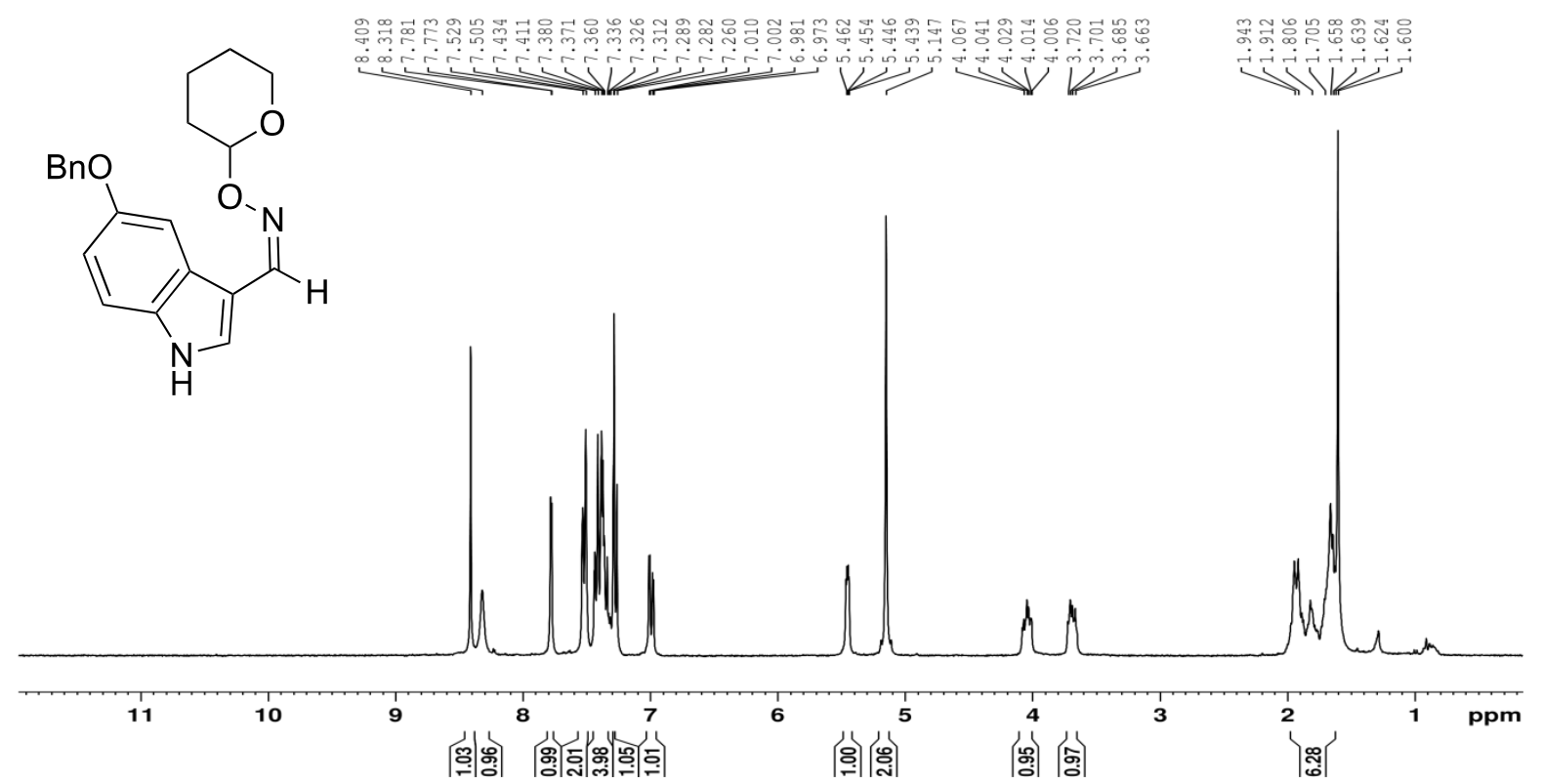

${ }^{13} \mathrm{C}\left\{{ }^{1} \mathrm{H}\right\}$ NMR $(75.5 \mathrm{MHz})$ Spectrum of $7 \mathbf{m}\left(\boldsymbol{Z}\right.$ isomer) in $d_{\underline{6}} \underline{\text {-DMSO }}$
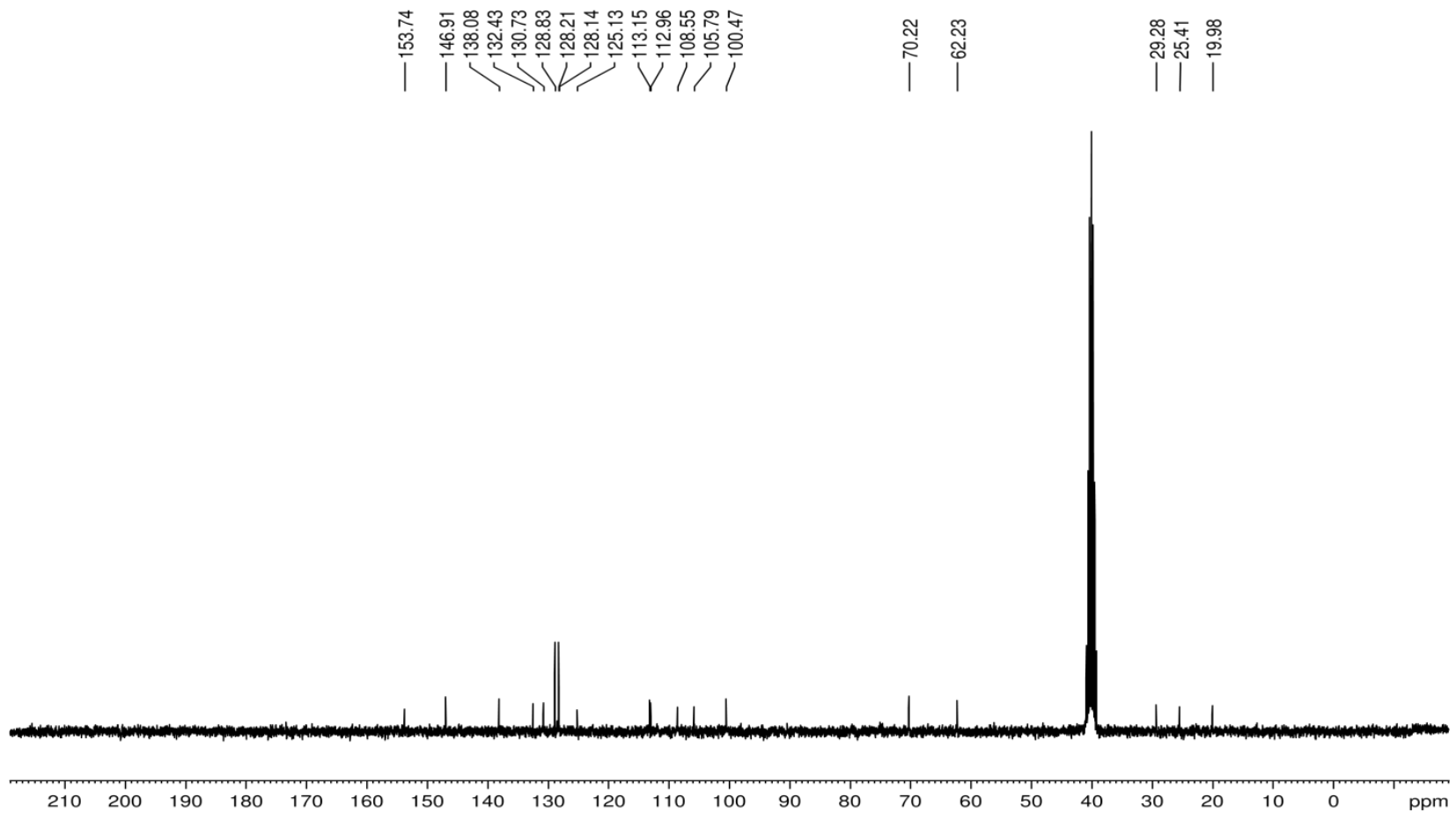
$\underline{{ }^{1} \mathrm{H} N M R}\left(300 \mathrm{MHz}\right.$ ) Spectrum of a 1: 8 Z/E mixture of $7 \mathbf{n}$ in $\mathrm{CDCl}_{3}$

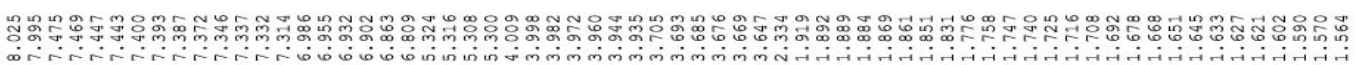

垈<smiles>C(=C/c1ccccc1)\N=C\OC1CCCCO1</smiles>

${ }^{13} \mathrm{C}\left\{{ }^{1} \mathrm{H}\right\}$ NMR (75.5 MHz) Spectrum of a 1: 8 Z/E mixture of $7 \mathbf{n}$ in $\mathrm{CDCl}_{3}$

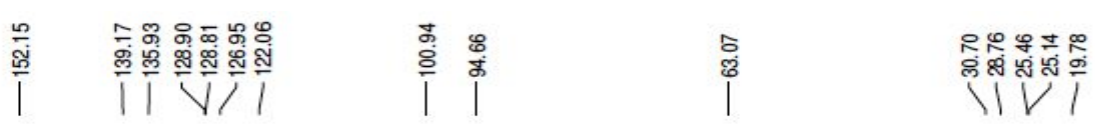

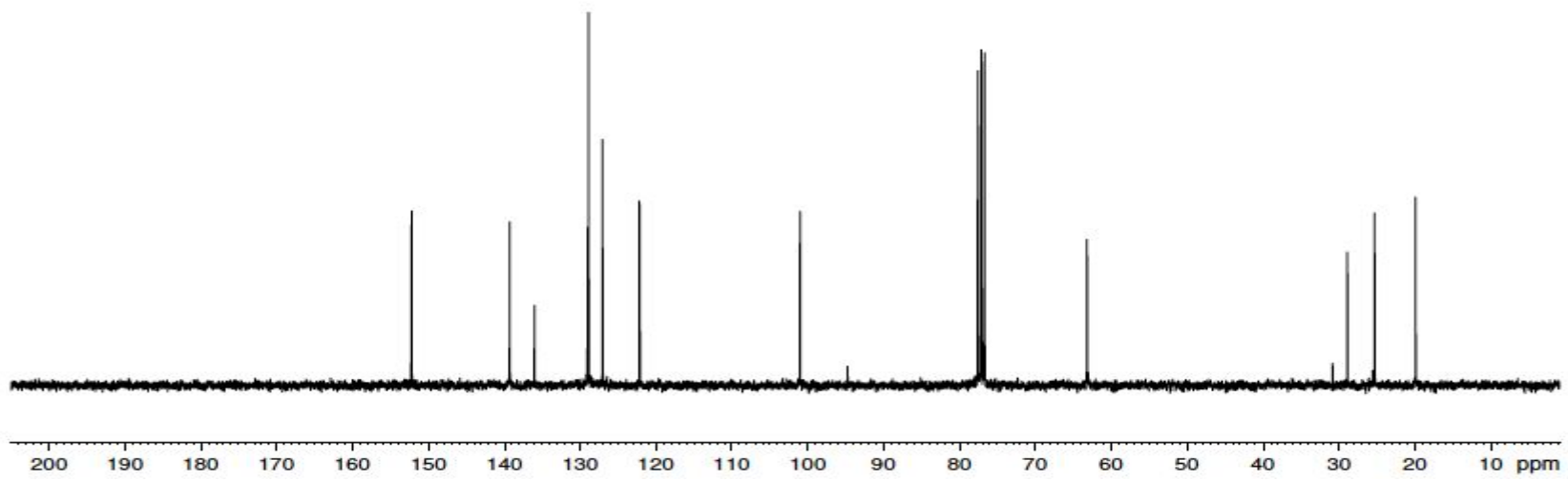


$\underline{{ }^{1} \mathrm{H} \text { NMR }(300 \mathrm{MHz}) \text { Spectrum of } 7 \mathbf{0}\left(\boldsymbol{E} \text { isomer) in } \mathrm{CDCl}_{3}\right.}$

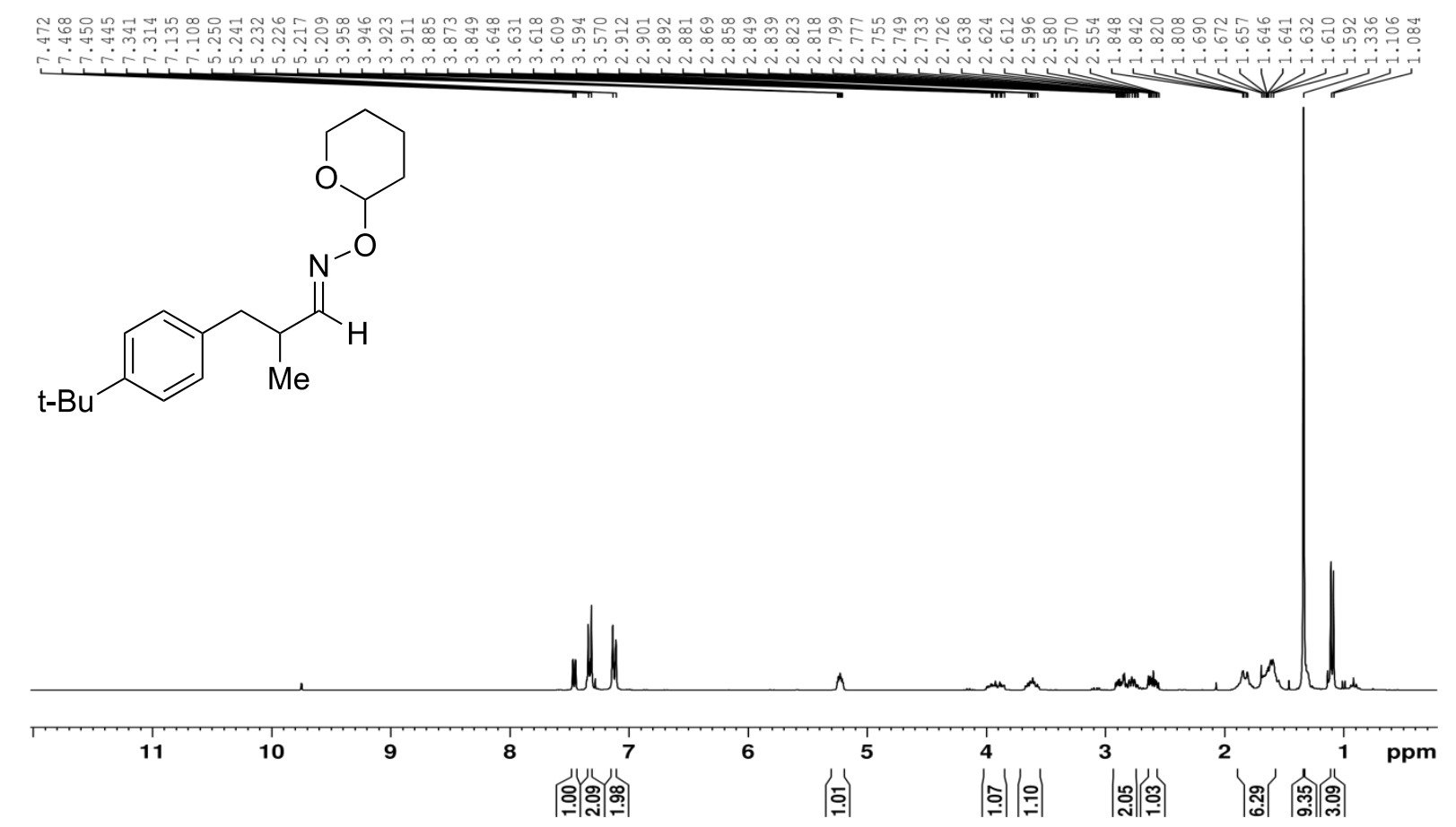

${ }^{13} \mathrm{C}\left\{{ }^{1} \mathrm{H}\right\}$ NMR (75.5 MHz) Spectrum of $7 \mathbf{0}\left(\boldsymbol{E}\right.$ isomer) in $\mathrm{CDCl}_{3}$
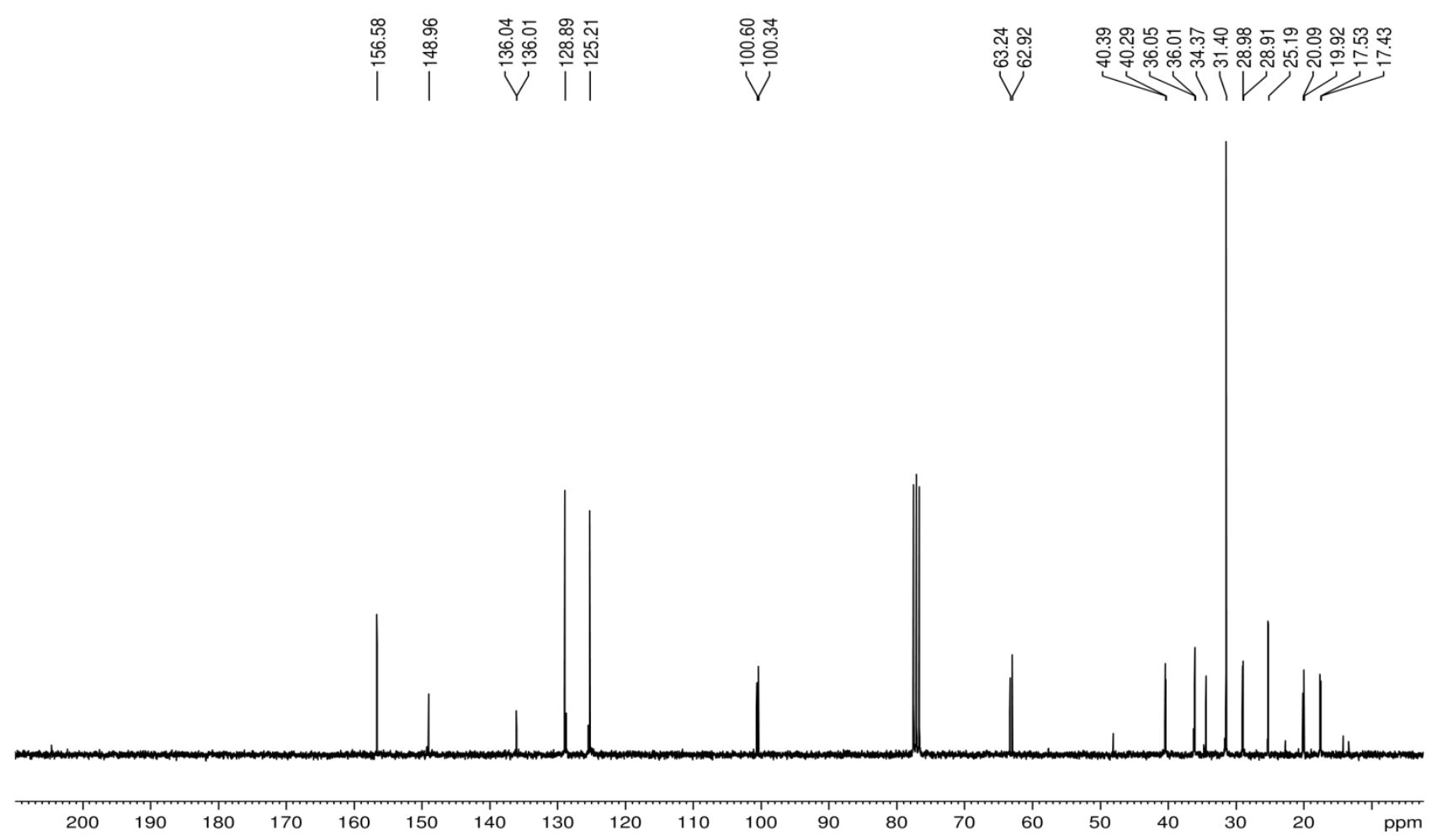


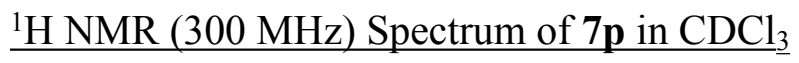

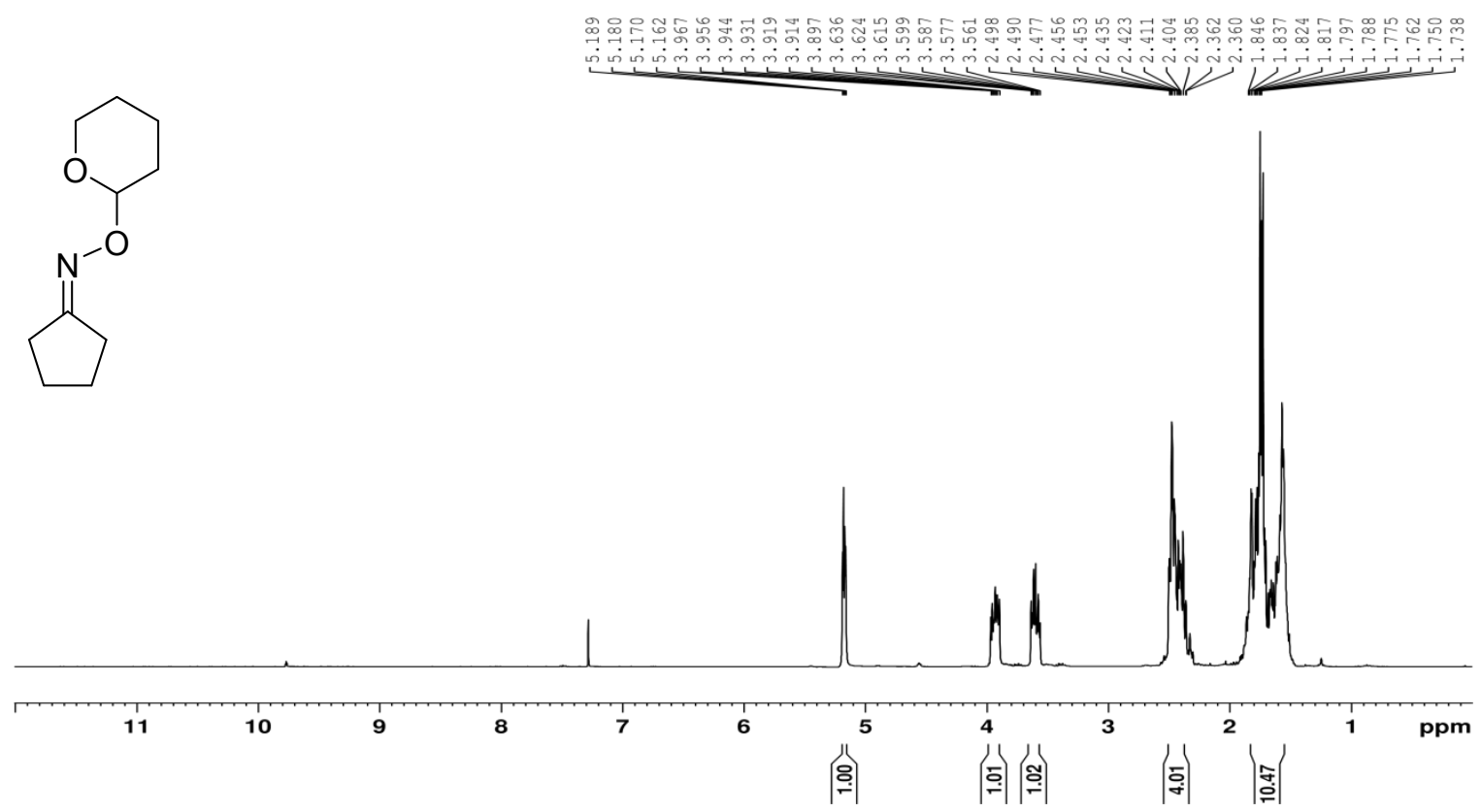

${ }^{13} \mathrm{C}\left\{{ }^{1} \mathrm{H}\right\}$ NMR (75.5 MHz) Spectrum of $7 \mathbf{p}$ in $\mathrm{CDCl}_{3}$

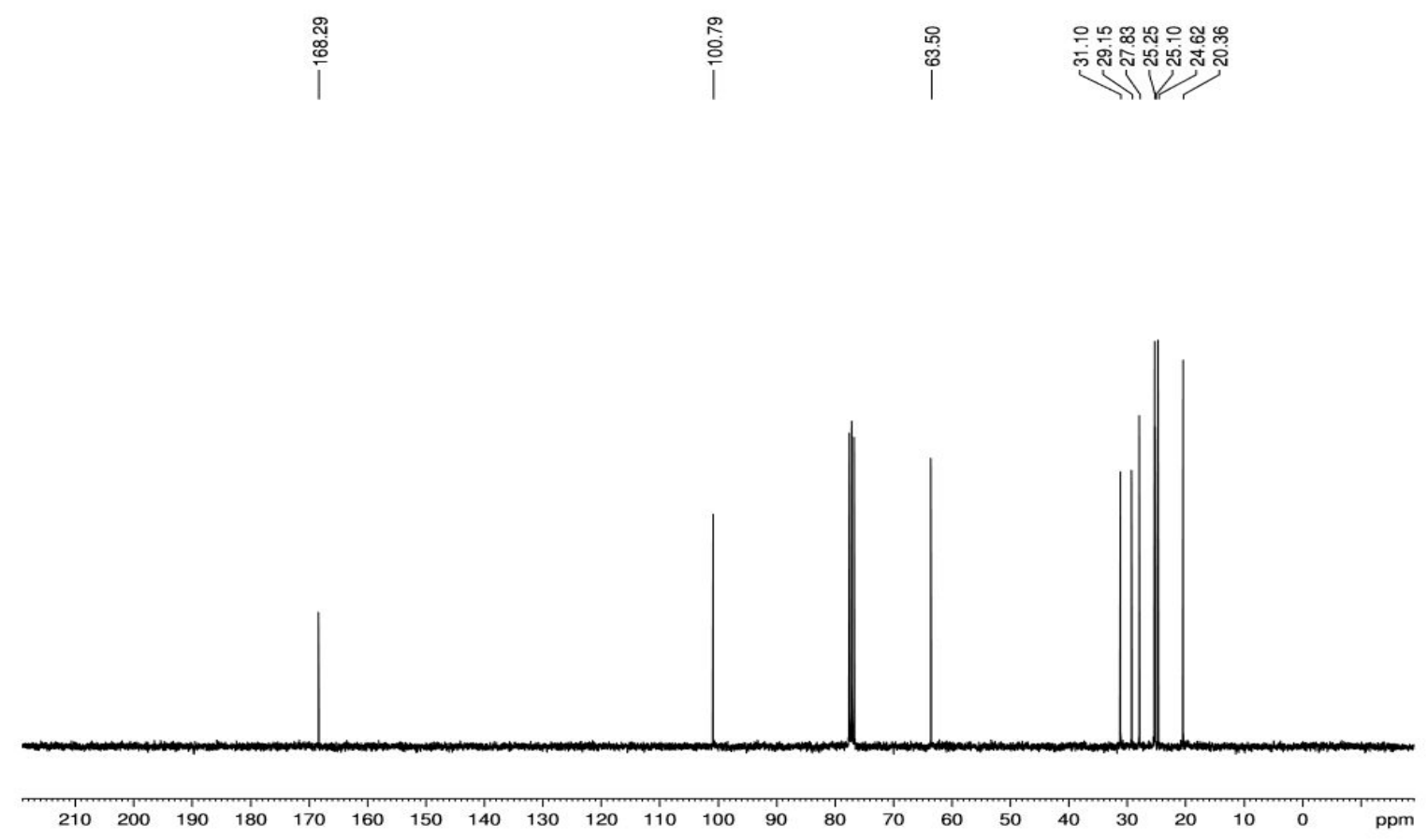


$\underline{{ }^{1} \mathrm{H} \text { NMR (300 MHz) Spectrum of } \mathbf{7 q} \text { in } \mathrm{CDCl}_{3}}$

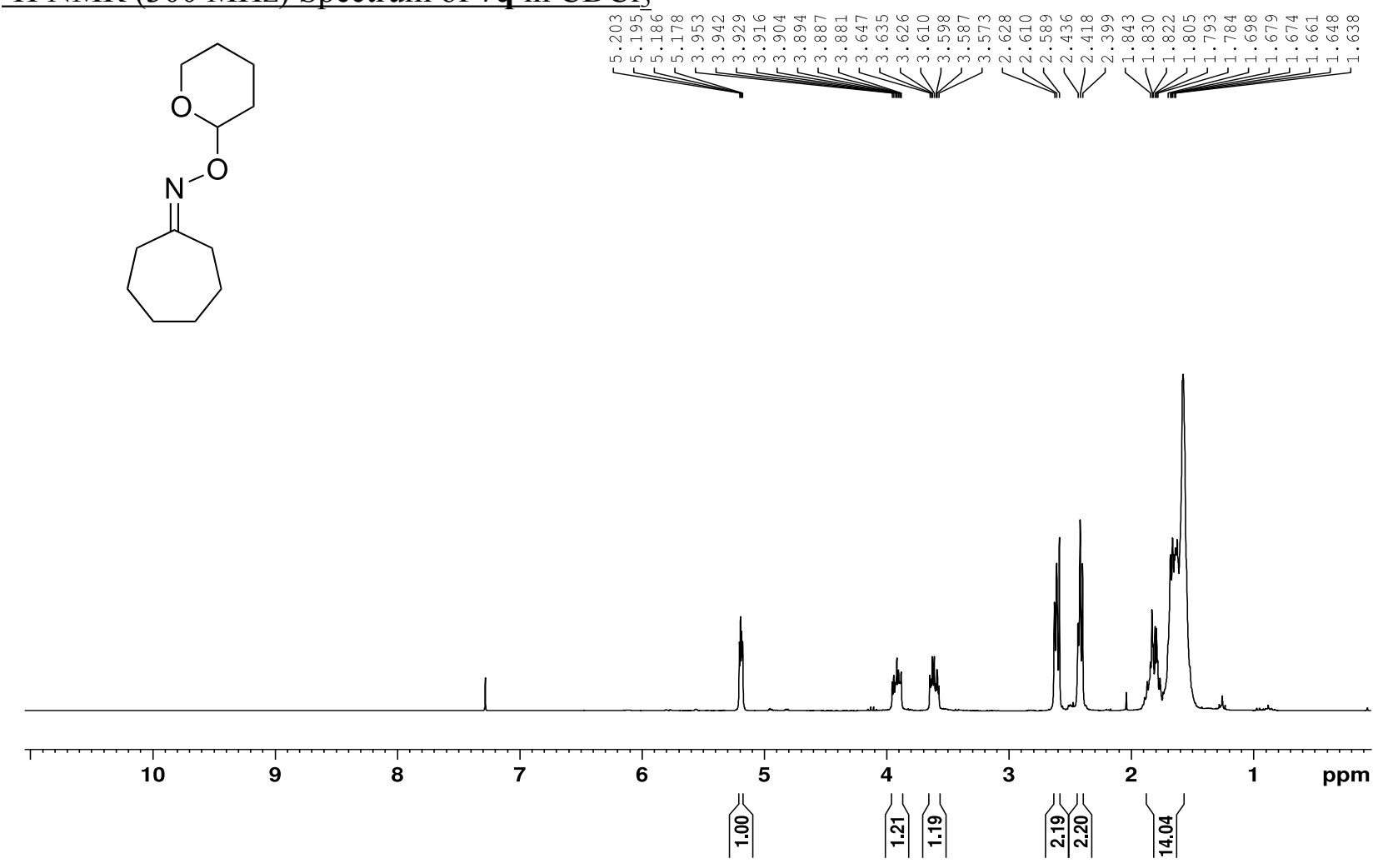

${ }^{13} \mathrm{C}\left\{{ }^{1} \mathrm{H}\right\}(75.5 \mathrm{MHz})$ NMR Spectrum of $7 \mathbf{q}$ in $\mathrm{CDCl}_{3}$
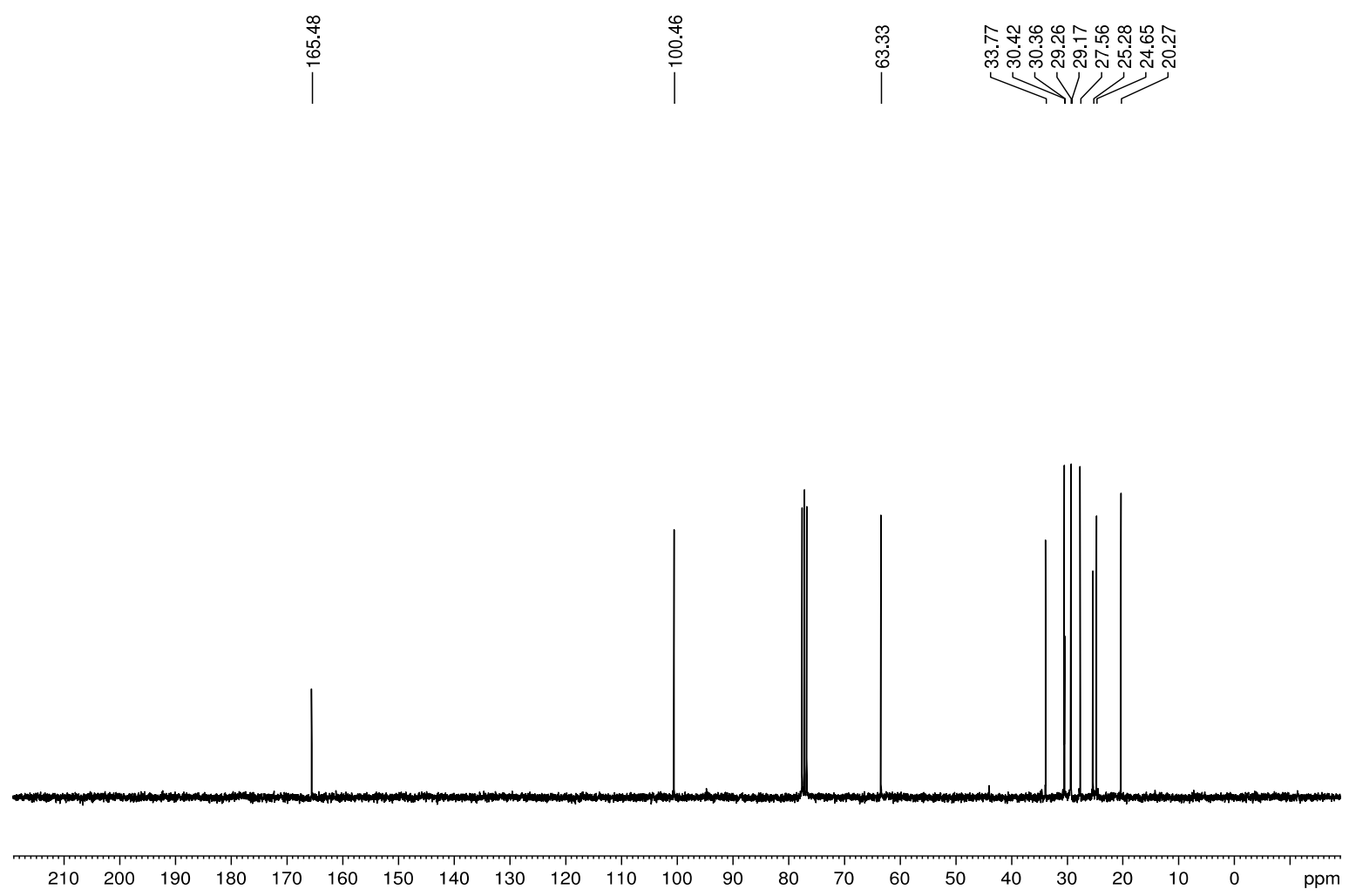
$\underline{{ }^{1} \mathrm{H} \mathrm{NMR}(300 \mathrm{MHz}) \text { Spectrum of a 1:2 Z/E mixture of } 7 \mathbf{r} \text { in } \mathrm{CDCl}_{3}}$

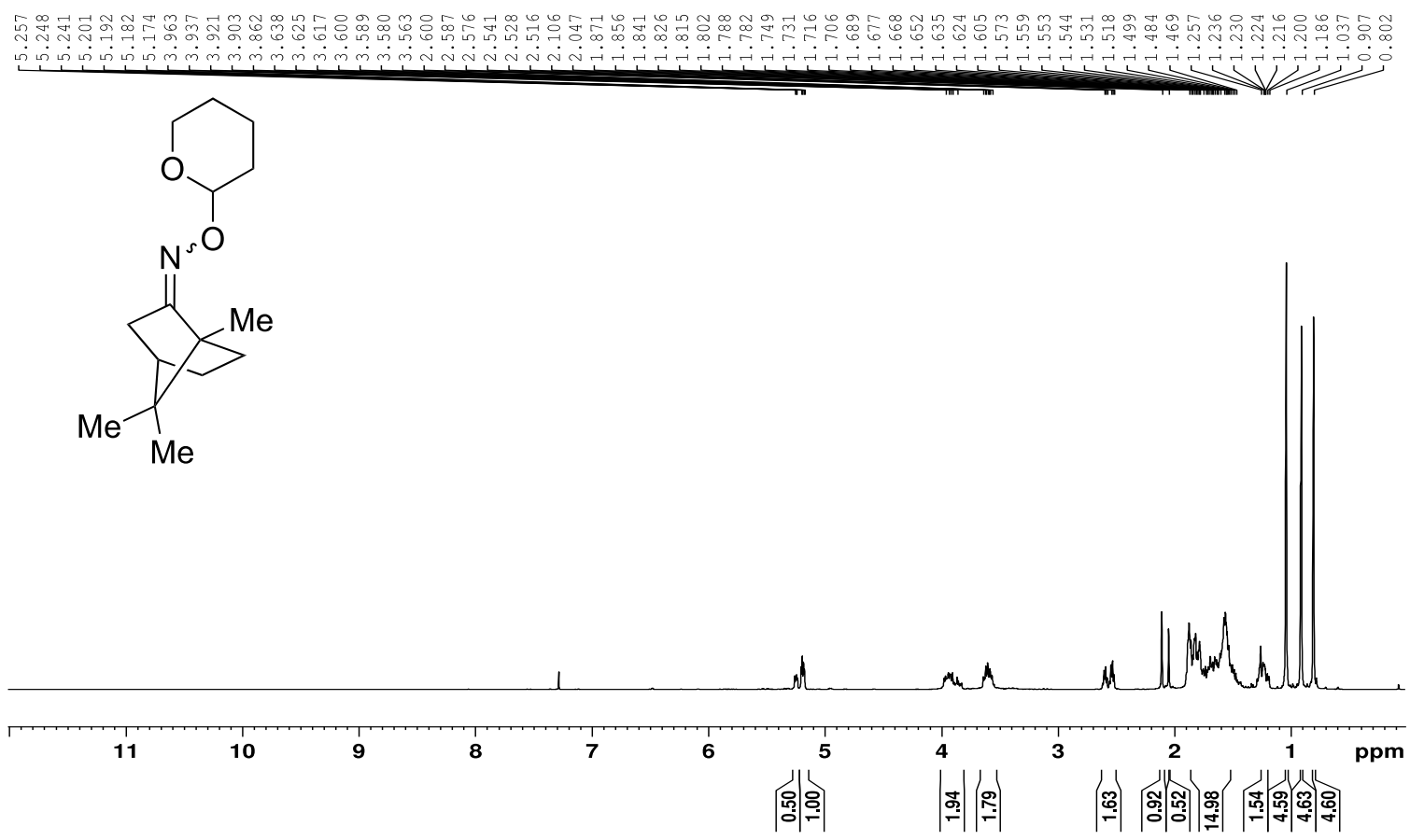

${ }^{{ }^{13} \mathrm{C}\left\{{ }^{1} \mathrm{H}\right\} \text { NMR (75.5 MHz) Spectrum of a 1:2 Z/E mixture of } 7 \mathbf{r} \text { in } \mathrm{CDCl}_{3}}$
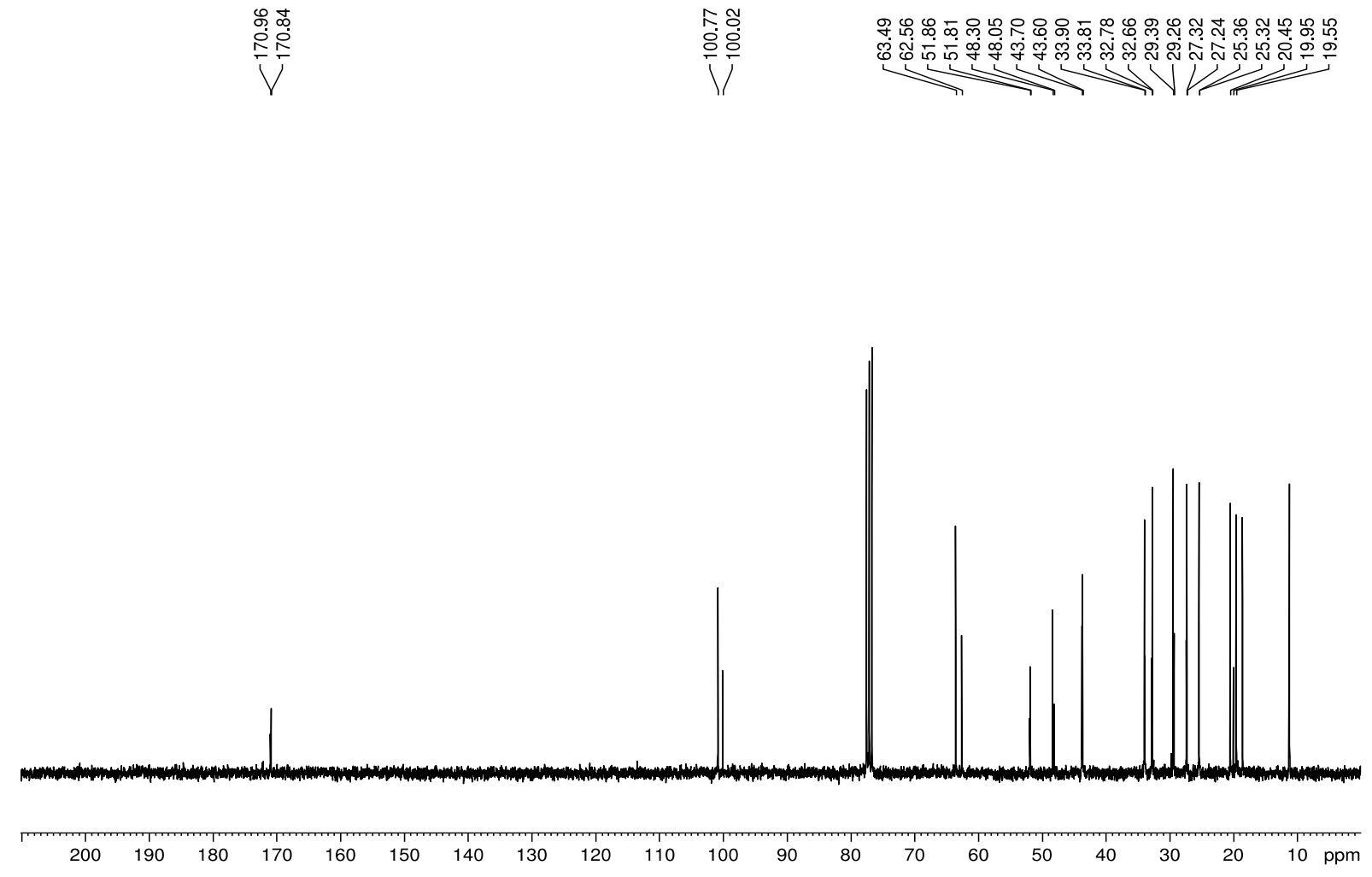
$\underline{{ }^{1} \mathrm{H} N M R}(300 \mathrm{MHz})$ Spectrum of $7 \mathbf{s}\left(\boldsymbol{E}\right.$ isomer) in $\mathrm{CDCl}_{3}$<smiles>CC(C)=CCCC1=CCC(/C=N/OC2CCCCO2)CC1</smiles>

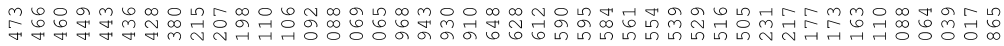

4
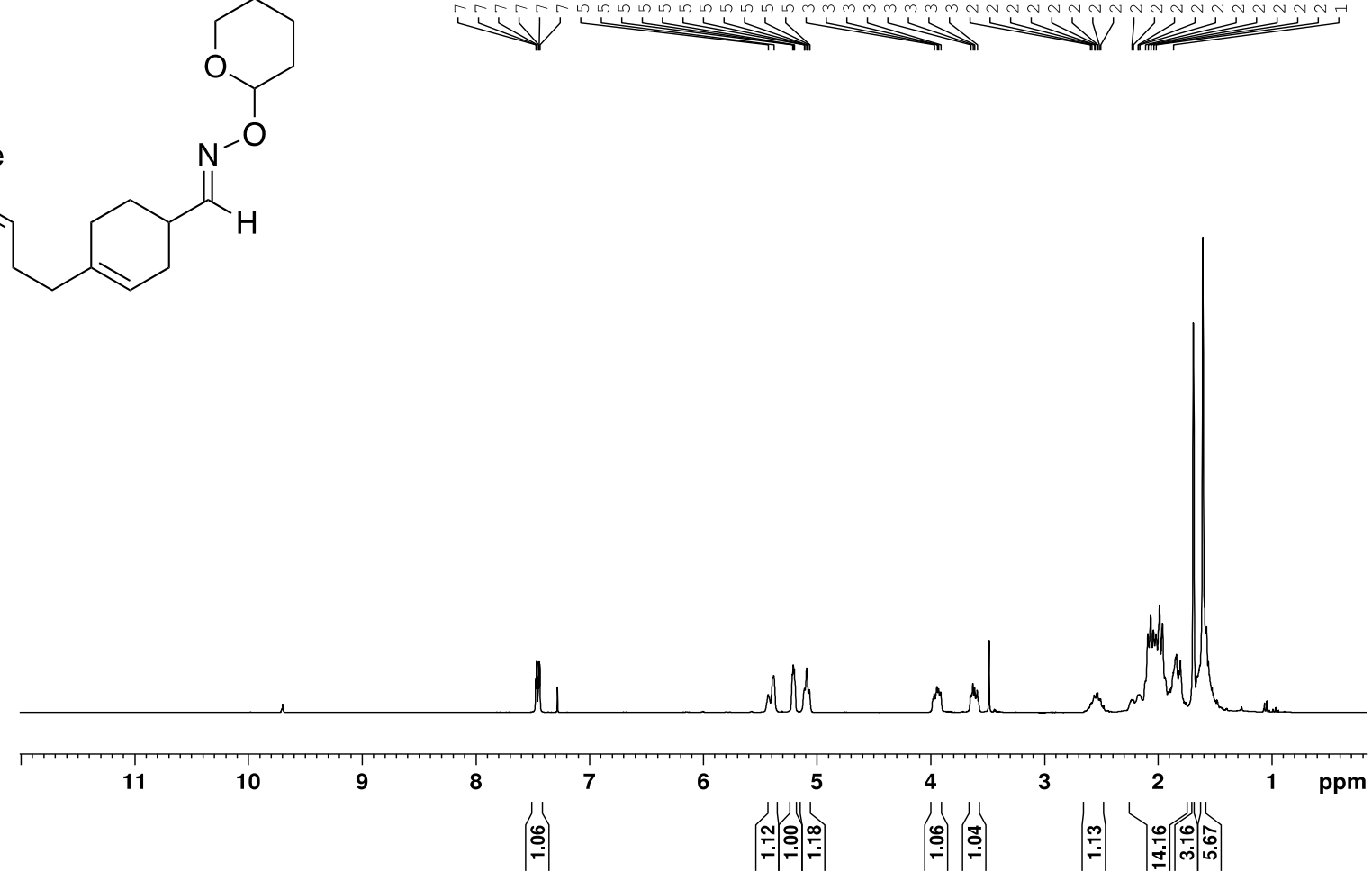

${ }^{13} \mathrm{C}\left\{{ }^{1} \mathrm{H}\right\}$ NMR (75.5 MHz) Spectrum of $7 \mathbf{s}\left(\boldsymbol{E}\right.$ isomer) in $\mathrm{CDCl}_{3}$

|

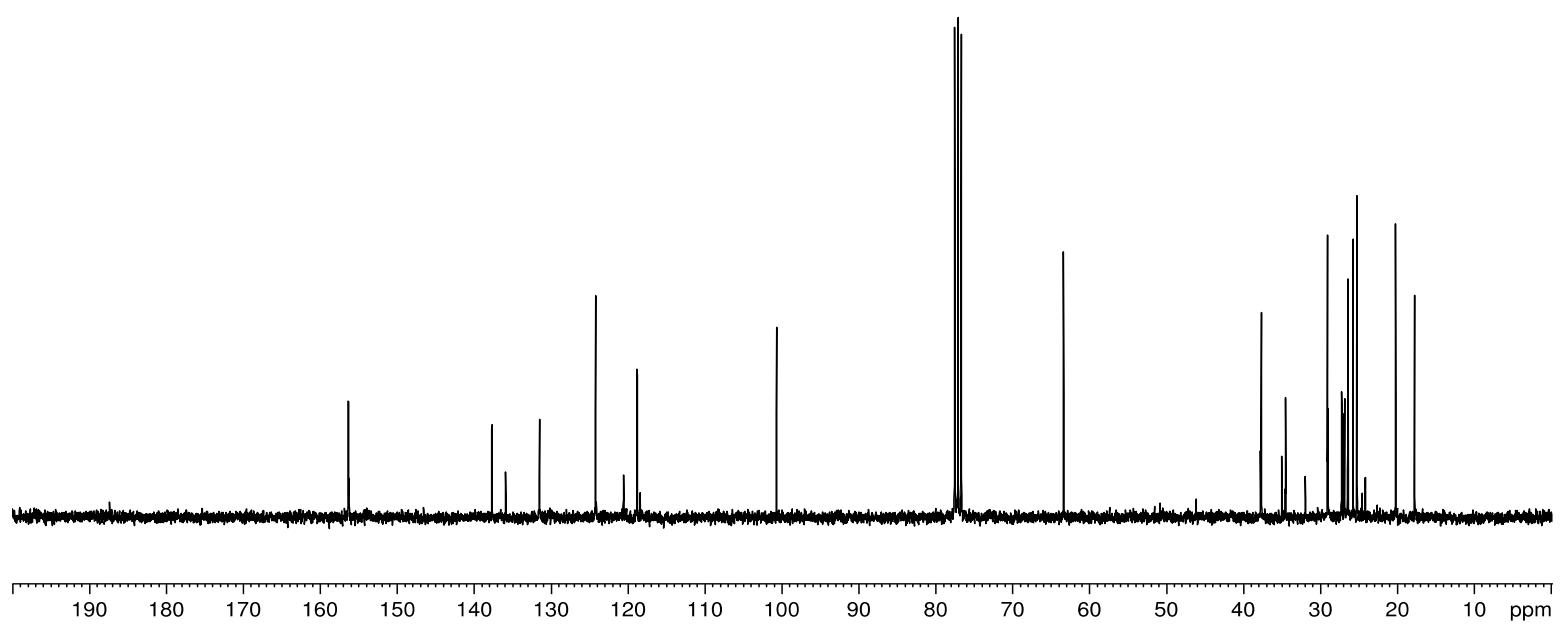


$\underline{{ }^{1} \mathrm{H} \mathrm{NMR}(300 \mathrm{MHz}) \text { Spectrum of } 7 \mathbf{s}\left(\boldsymbol{Z} \text { isomer) in } \mathrm{CDCl}_{3}\right.}$

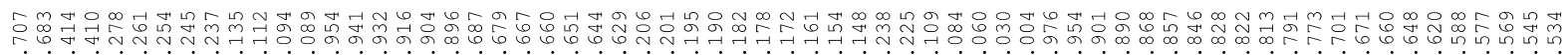

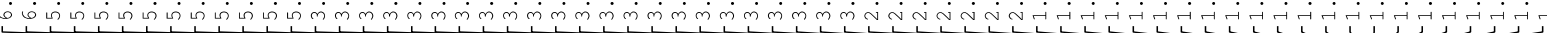<smiles>CC(C)=CCCC1=CCC(/C=N/OC2CCCCO2)CC1</smiles>

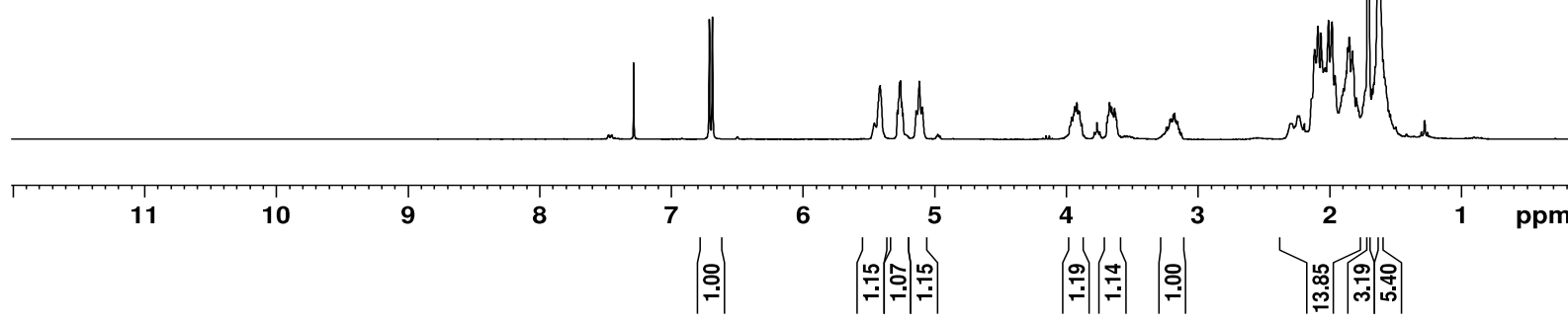

${ }^{13} \mathrm{C}\left\{{ }^{1} \mathrm{H}\right\}$ NMR (75.5 MHz) Spectrum of $7 \mathbf{s}\left(\boldsymbol{Z}\right.$ isomer) in $\mathrm{CDCl}_{3}$

V|l
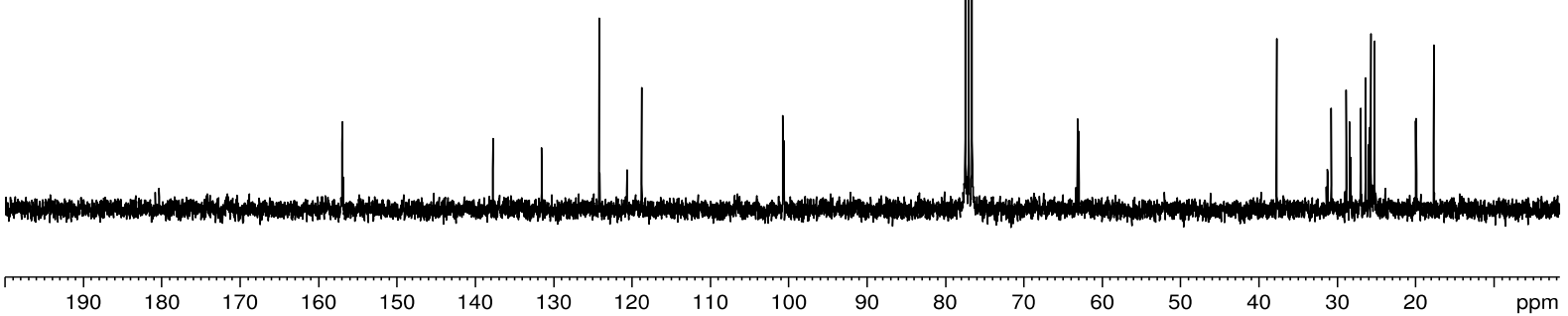
$\underline{{ }^{1} \mathrm{H} \text { NMR (300 MHz) Spectrum of 7t (C.N.D.) in } \mathrm{CDCl}_{3}}$
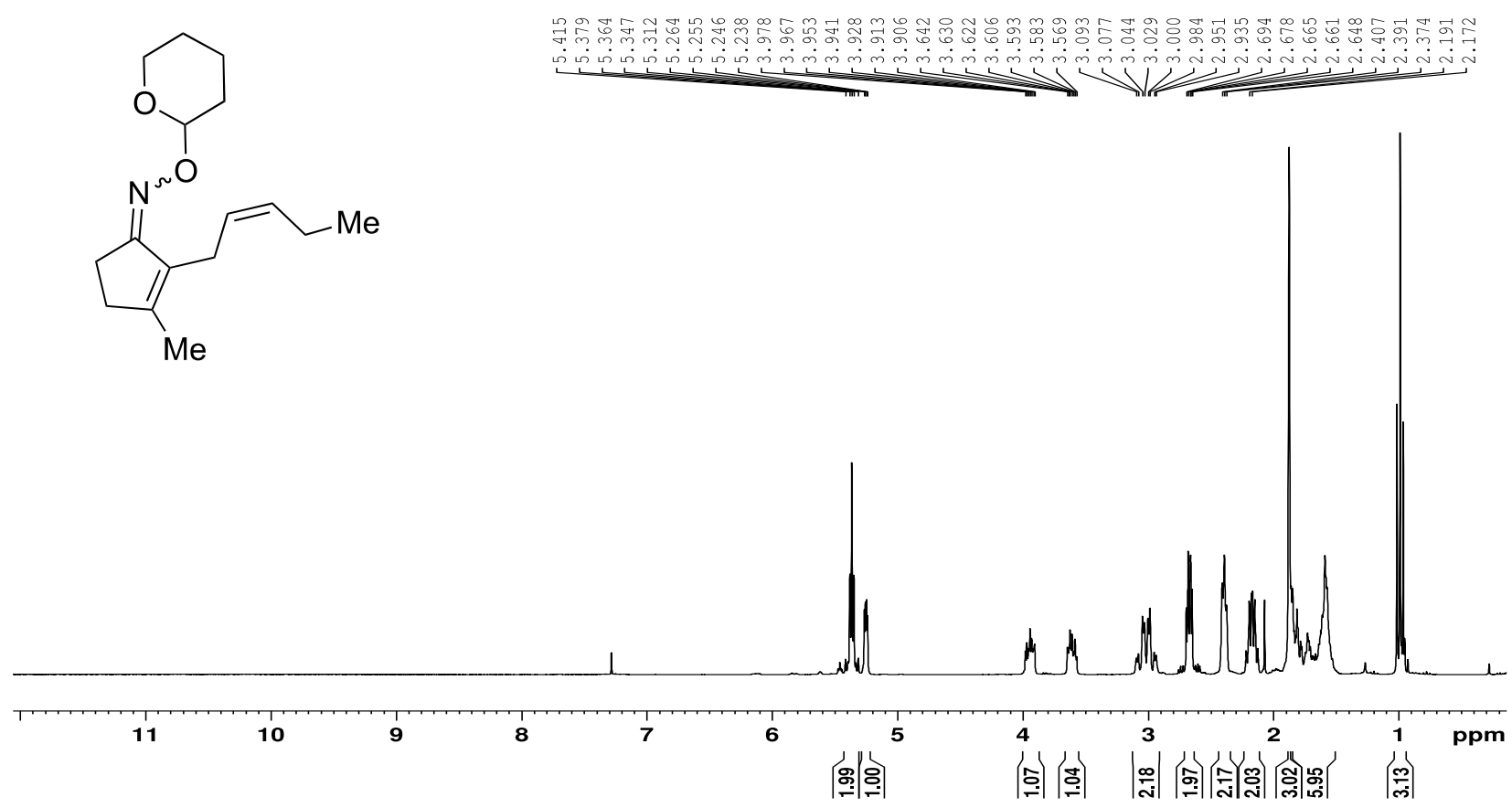

${ }^{13} \mathrm{C}\left\{{ }^{1} \mathrm{H}\right\}$ NMR (75.5 MHz) Spectrum of $7 \mathbf{t}$ (C.N.D.) in $\mathrm{CDCl}_{3}$

\begin{tabular}{|c|c|c|c|c|}
\hline 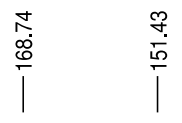 & 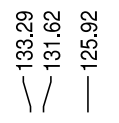 & 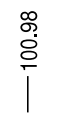 & $\frac{\infty}{\dot{0}}$ & 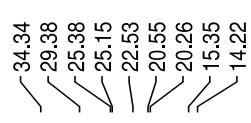 \\
\hline
\end{tabular}
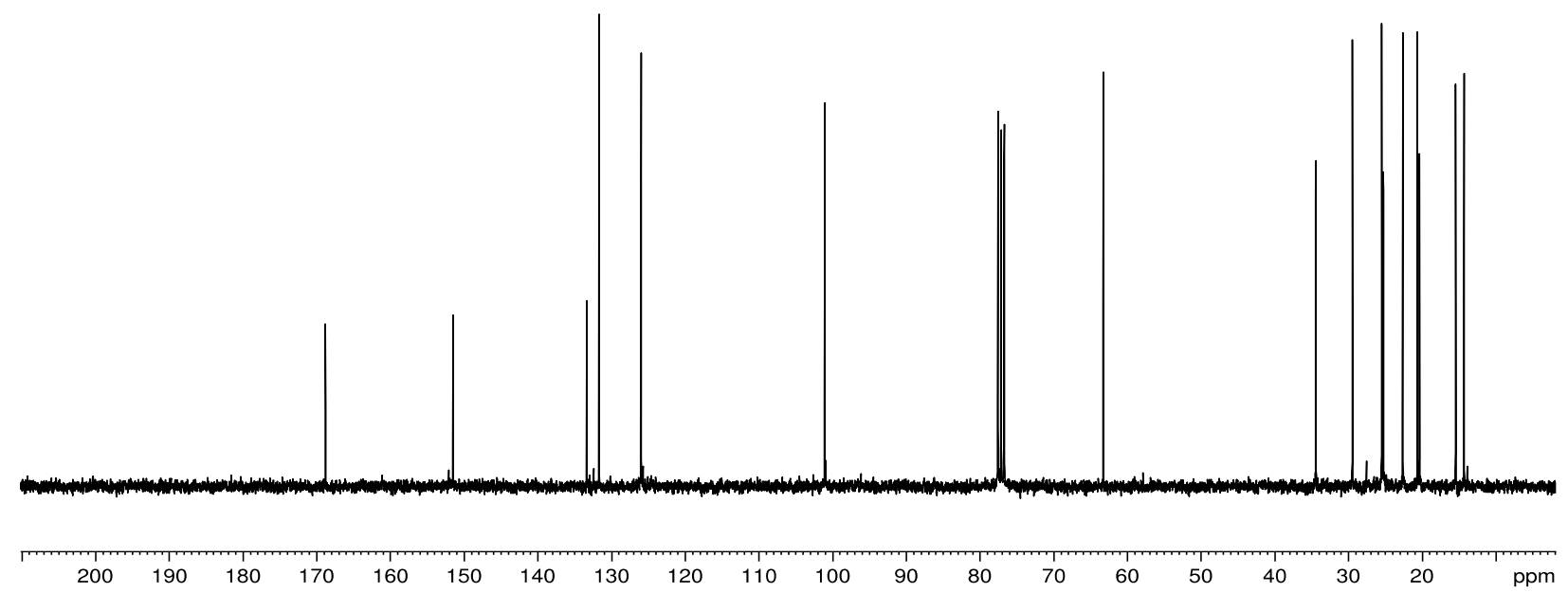
$\underline{{ }^{1} \mathrm{H} \mathrm{NMR}(300 \mathrm{MHz}) \text { Spectrum of a 1:5 Z/E mixture of } 7 \mathbf{u} \text { in } \mathrm{CDCl}_{3}}$

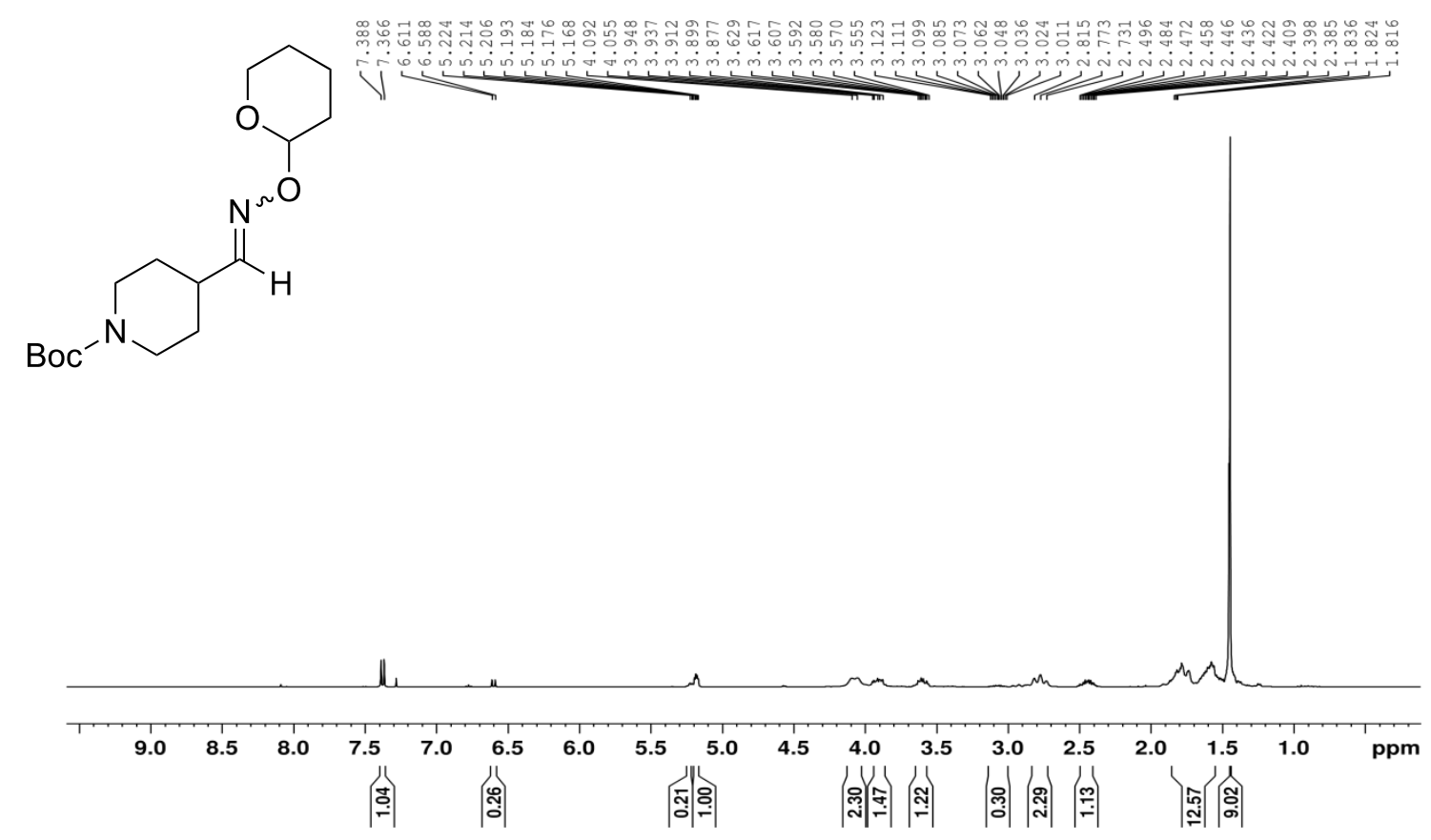

${ }^{13} \mathrm{C}\left\{{ }^{1} \mathrm{H}\right\}$ NMR (75.5 MHz) Spectrum of a 1:5 Z/E mixture of $7 \mathbf{u}$ in $\mathrm{CDCl}_{3}$

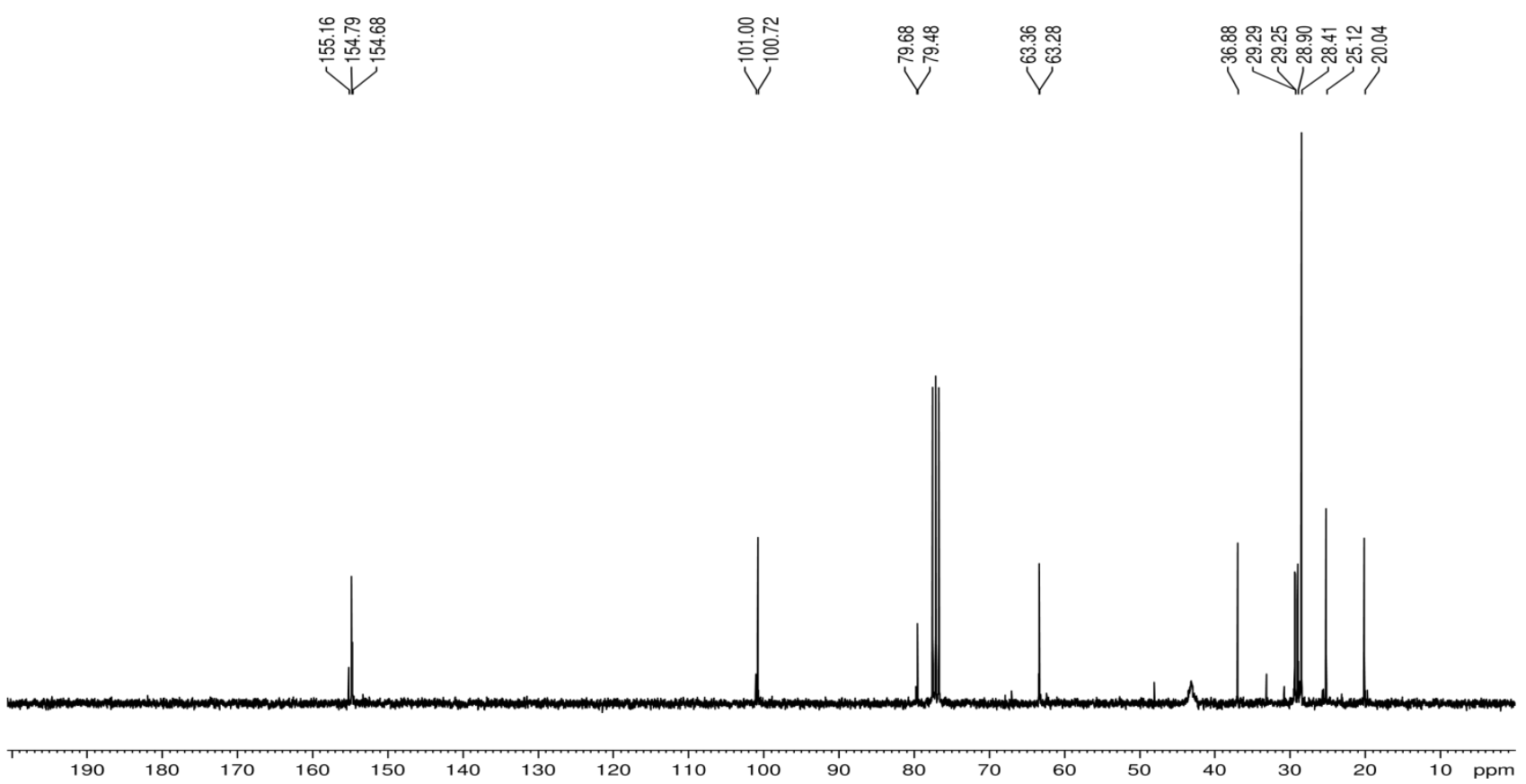


$\underline{{ }^{1} \mathrm{H} \text { NMR (300 MHz) Spectrum of a 1:2 Z/E mixture of } 7 \mathbf{v} \text { in } \mathrm{CDCl}_{3}}$

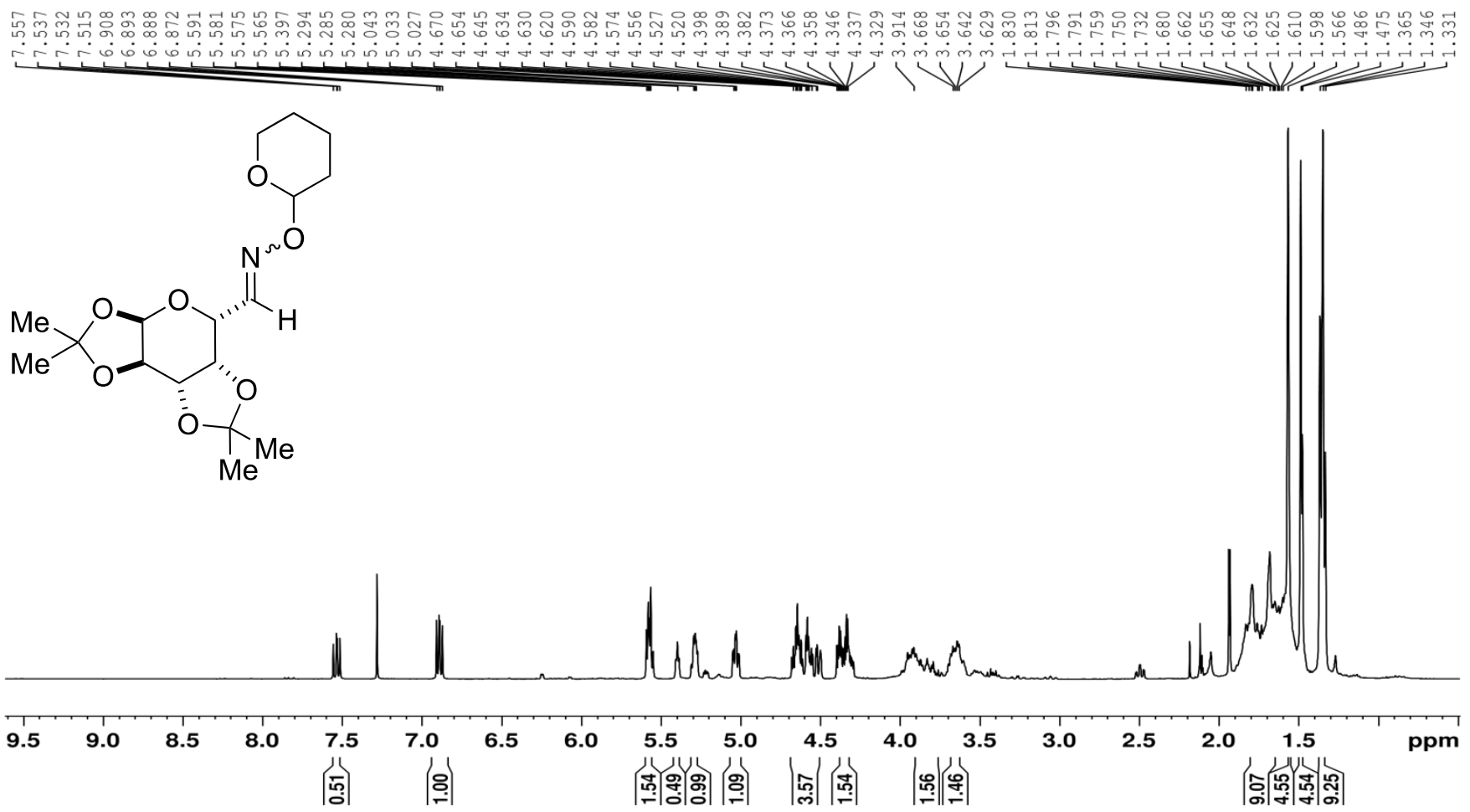

${ }^{13} \mathrm{C}\left\{{ }^{1} \mathrm{H}\right\}$ NMR (75.5 MHz) Spectrum of a 1:2 Z/E mixture of $7 \mathbf{v}$ in $\mathrm{CDCl}_{\underline{3}}$

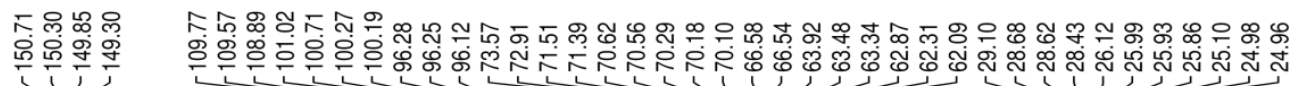

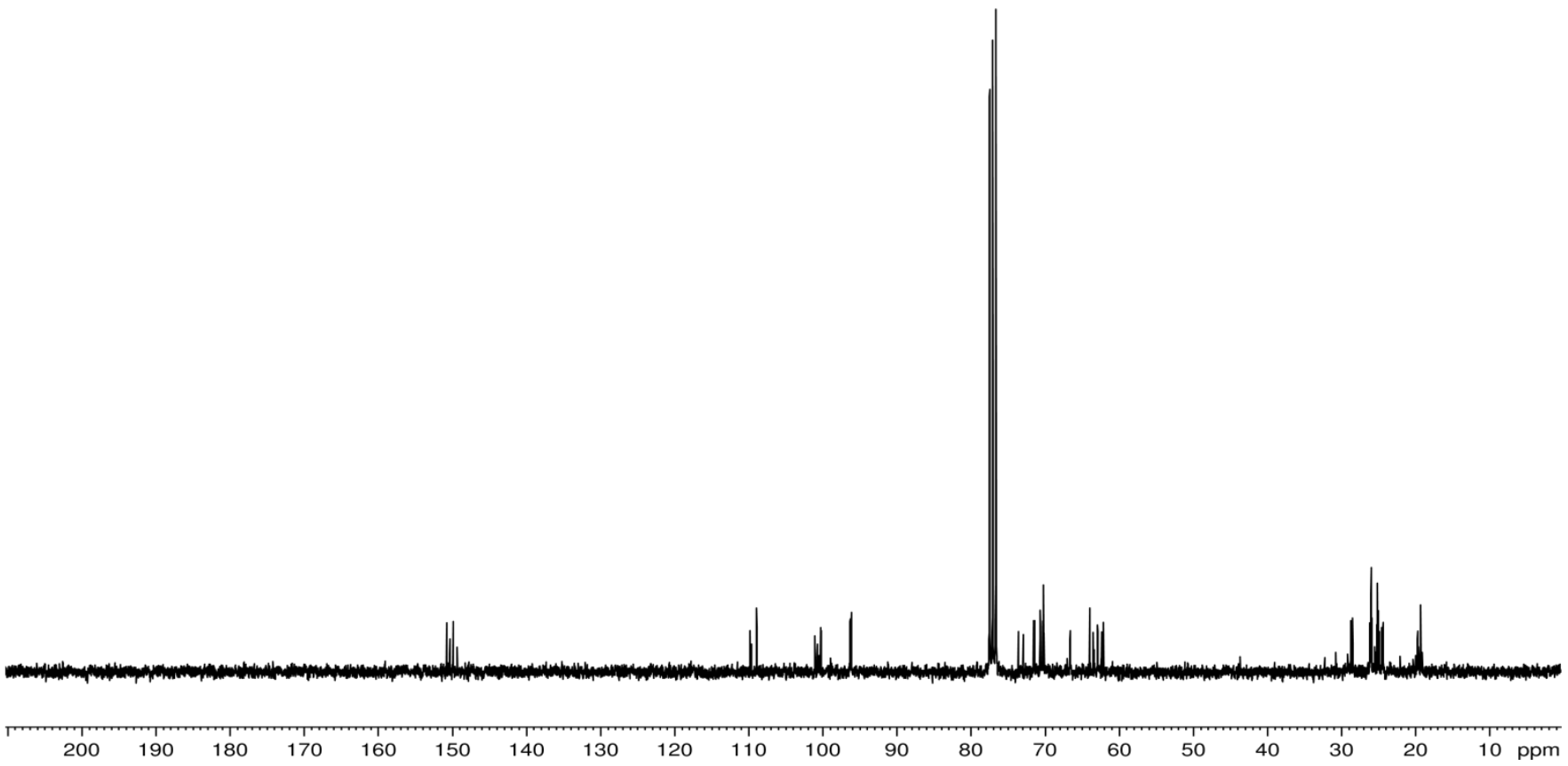


$\underline{{ }^{1} \mathrm{H} N M R}(300 \mathrm{MHz})$ Spectrum of $7 \mathbf{w}\left(\boldsymbol{E}\right.$ isomer) in $\mathrm{CDCl}_{3}$

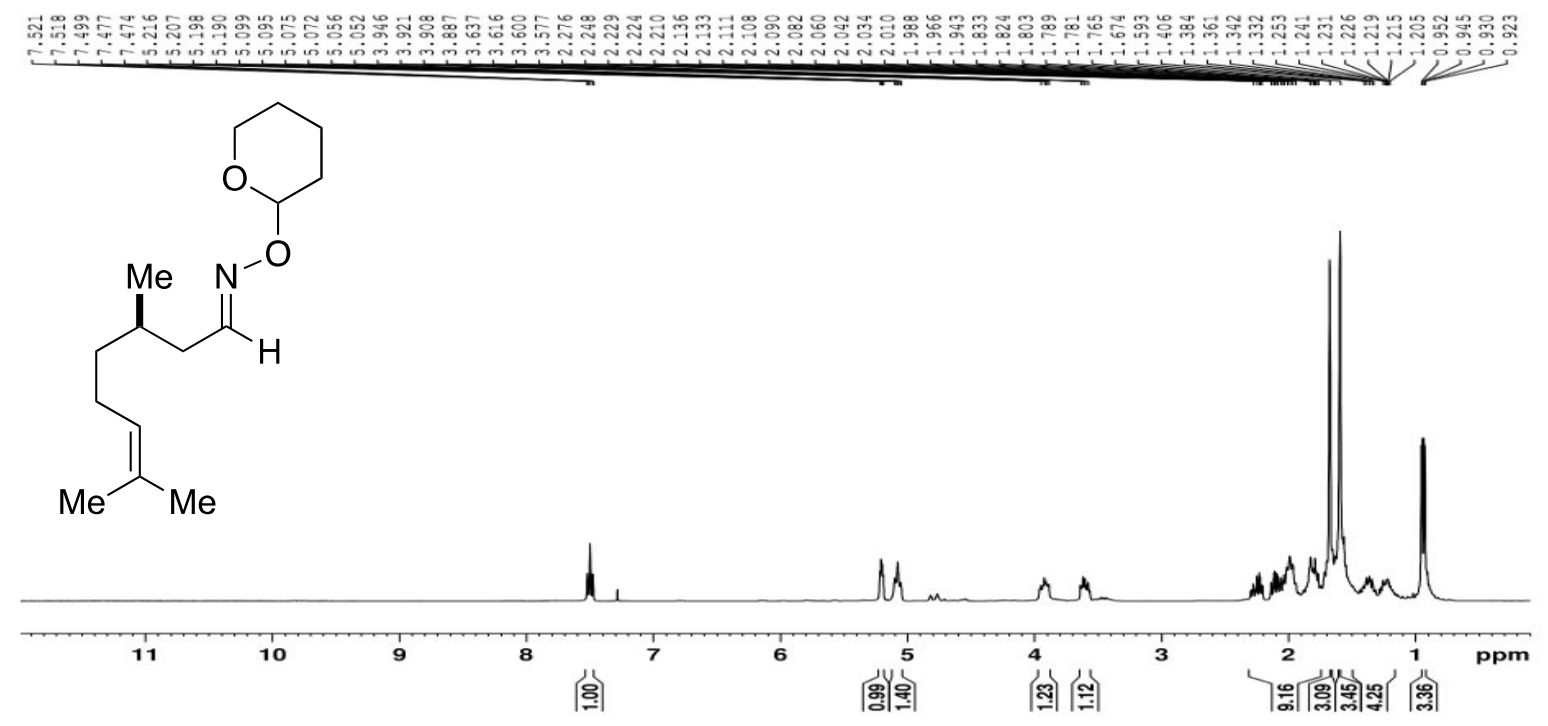

${ }^{13} \mathrm{C}\left\{{ }^{1} \mathrm{H}\right\}$ NMR (75.5 MHz) Spectrum of $7 \mathbf{w}\left(\boldsymbol{E}\right.$ isomer) in $\mathrm{CDCl}_{3}$

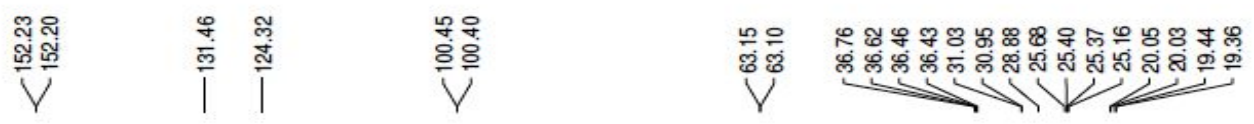
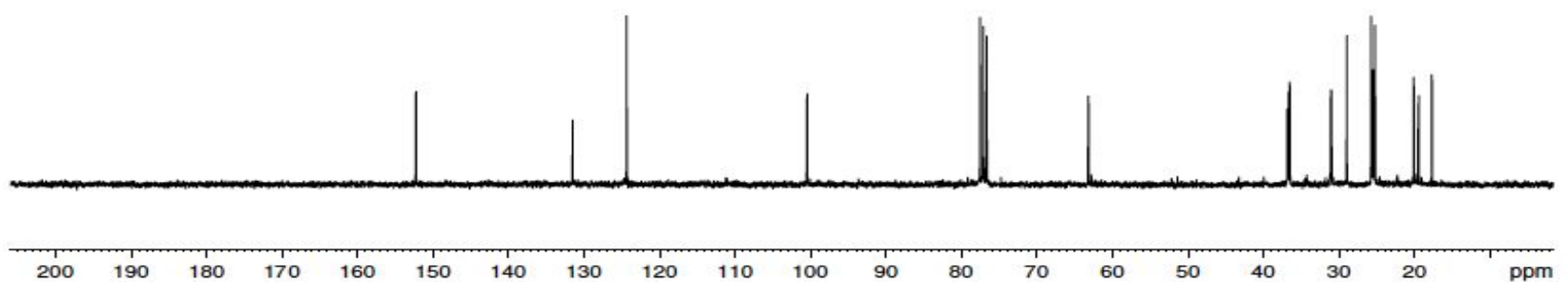
$\underline{{ }^{1} \mathrm{H} \text { NMR }(300 \mathrm{MHz}) \text { Spectrum of } 7 \mathbf{x}\left(\boldsymbol{E} \text { isomer) in } \mathrm{CDCl}_{3}\right.}$<smiles>CCOC(=O)/C(N)=N/OC1CCCCO1</smiles>

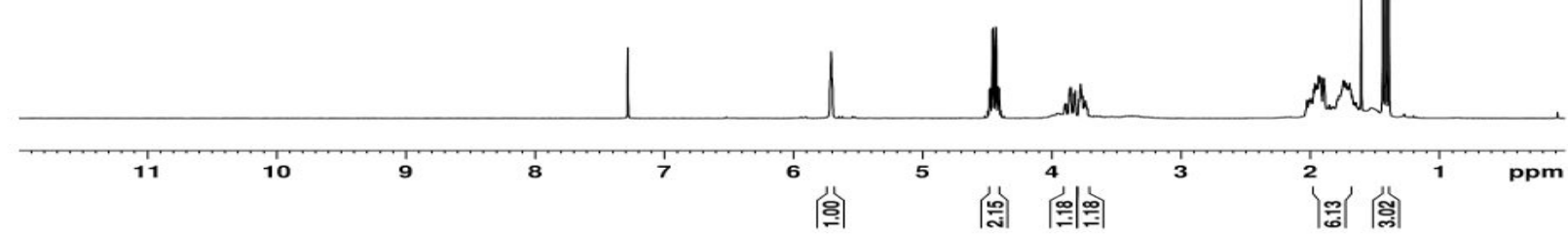

${ }^{13} \mathrm{C}\left\{{ }^{1} \mathrm{H}\right\}$ NMR (75.5 MHz) Spectrum of $7 \mathbf{x}\left(\boldsymbol{E}\right.$ isomer) in $\mathrm{CDCl}_{3}$

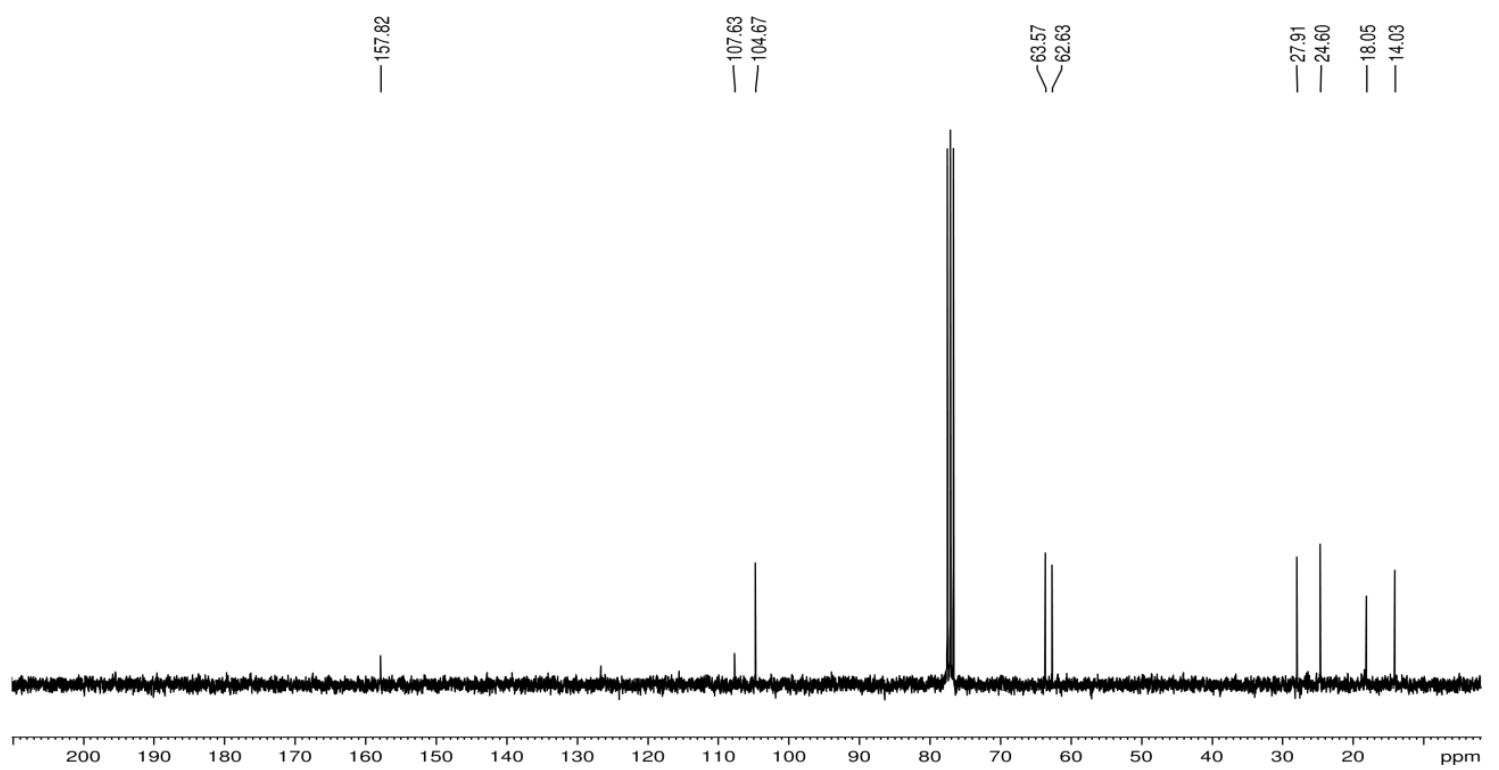




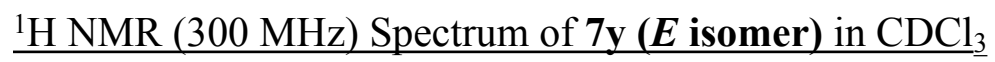
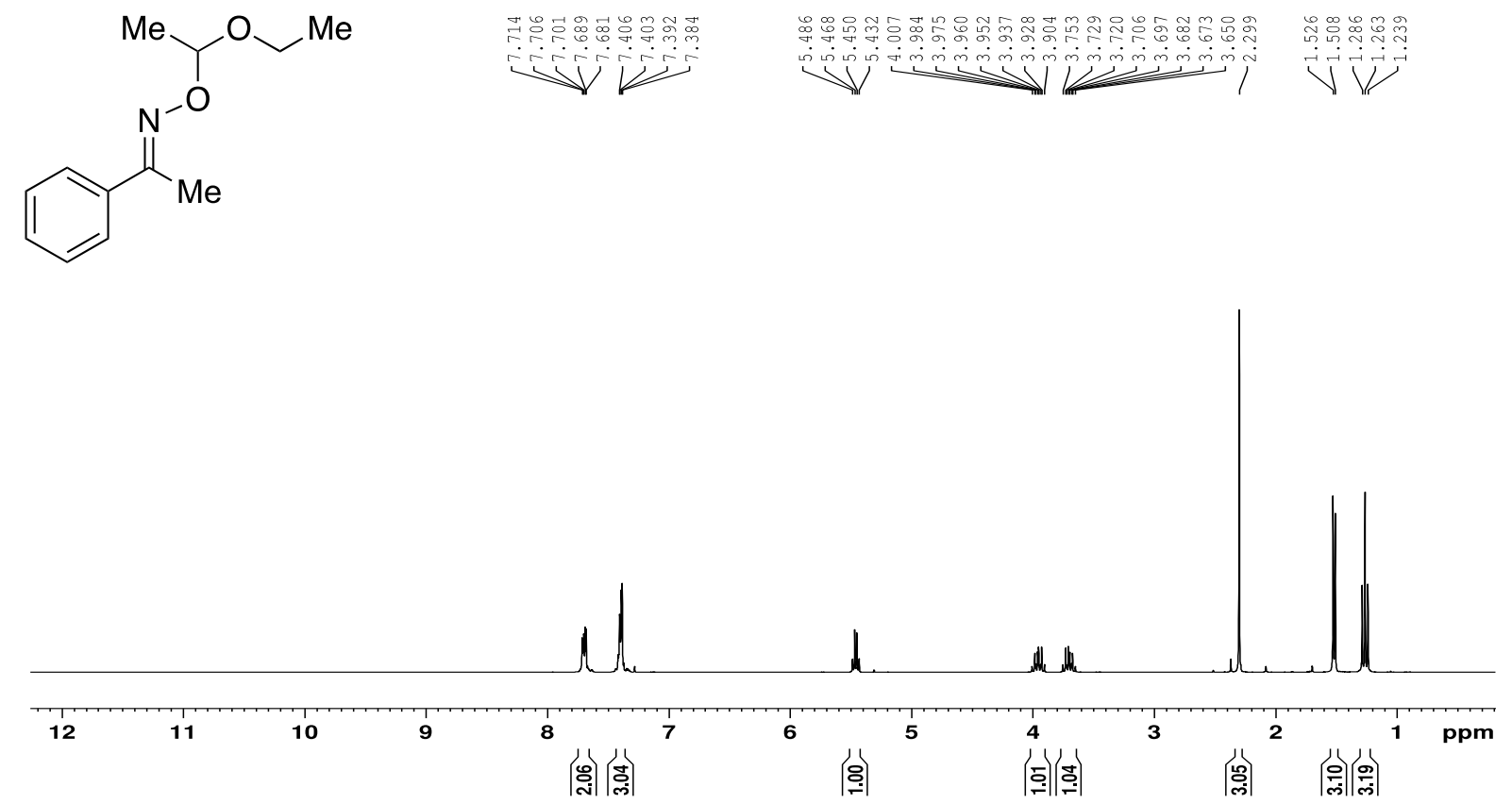

${ }^{13} \mathrm{C}\left\{{ }^{1} \mathrm{H}\right\}$ NMR (75.5 MHz) Spectrum of $7 \mathbf{y}\left(\boldsymbol{E}\right.$ isomer) in $\mathrm{CDCl}_{3}$
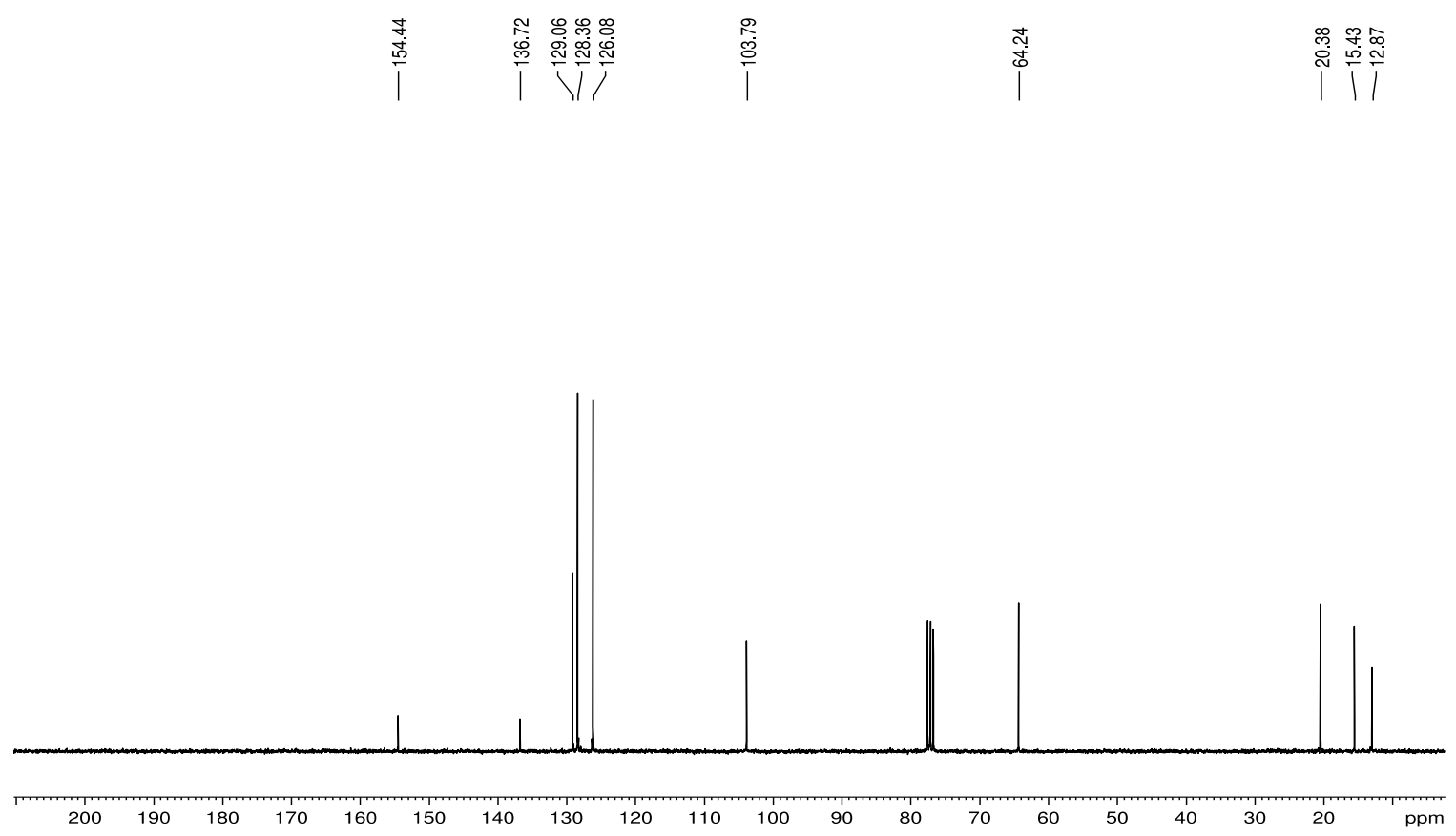


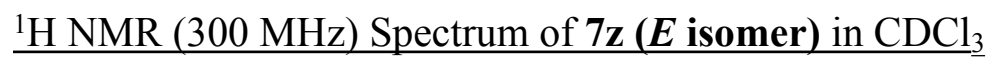<smiles>CCCCOC(C)O/N=C(\C)c1ccccc1</smiles>
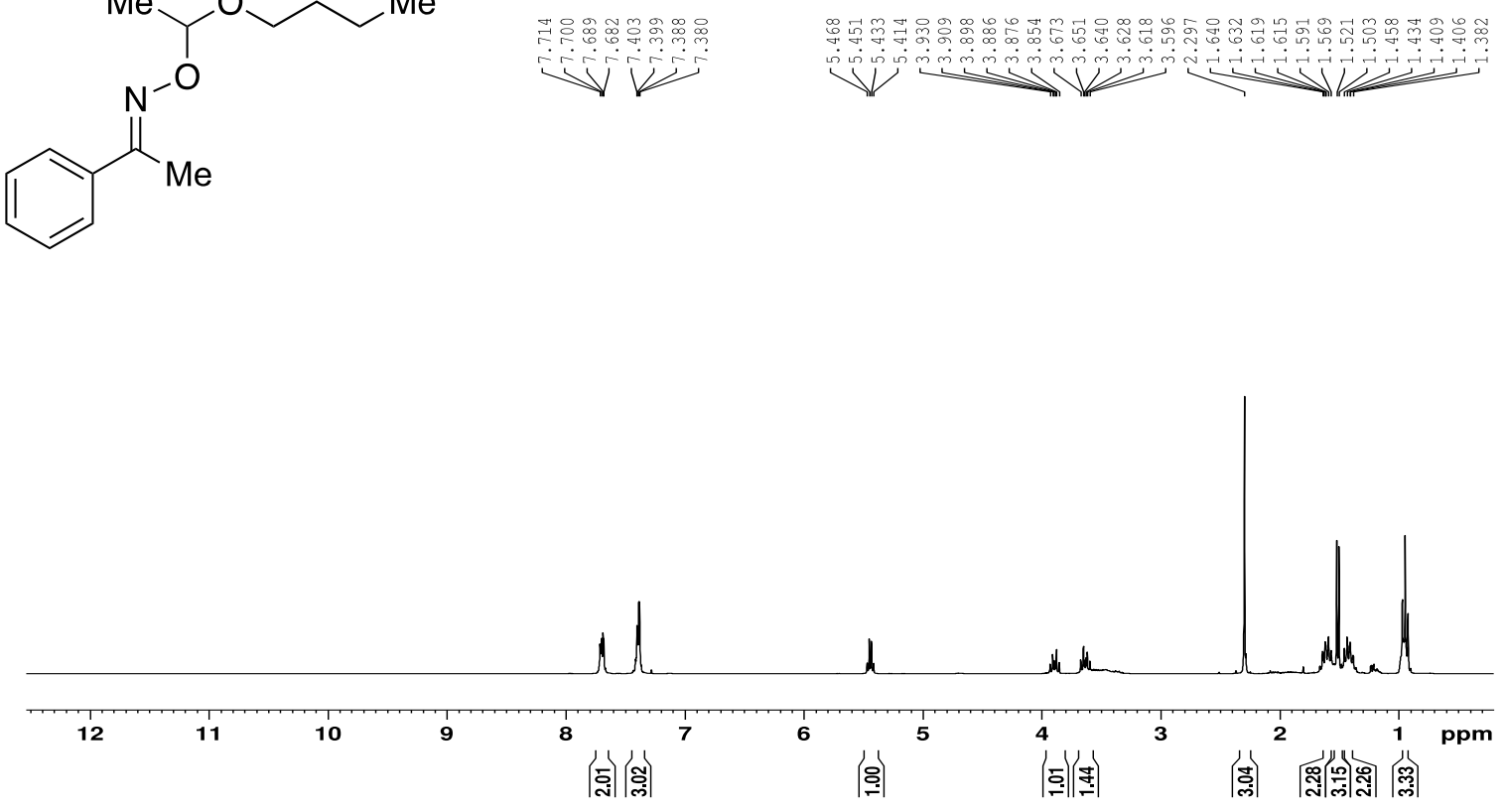

${ }^{13} \mathrm{C}\left\{{ }^{1} \mathrm{H}\right\}$ NMR (75.5 MHz) Spectrum of $7 \mathbf{z}\left(\boldsymbol{E}\right.$ isomer) in $\mathrm{CDCl}_{3}$

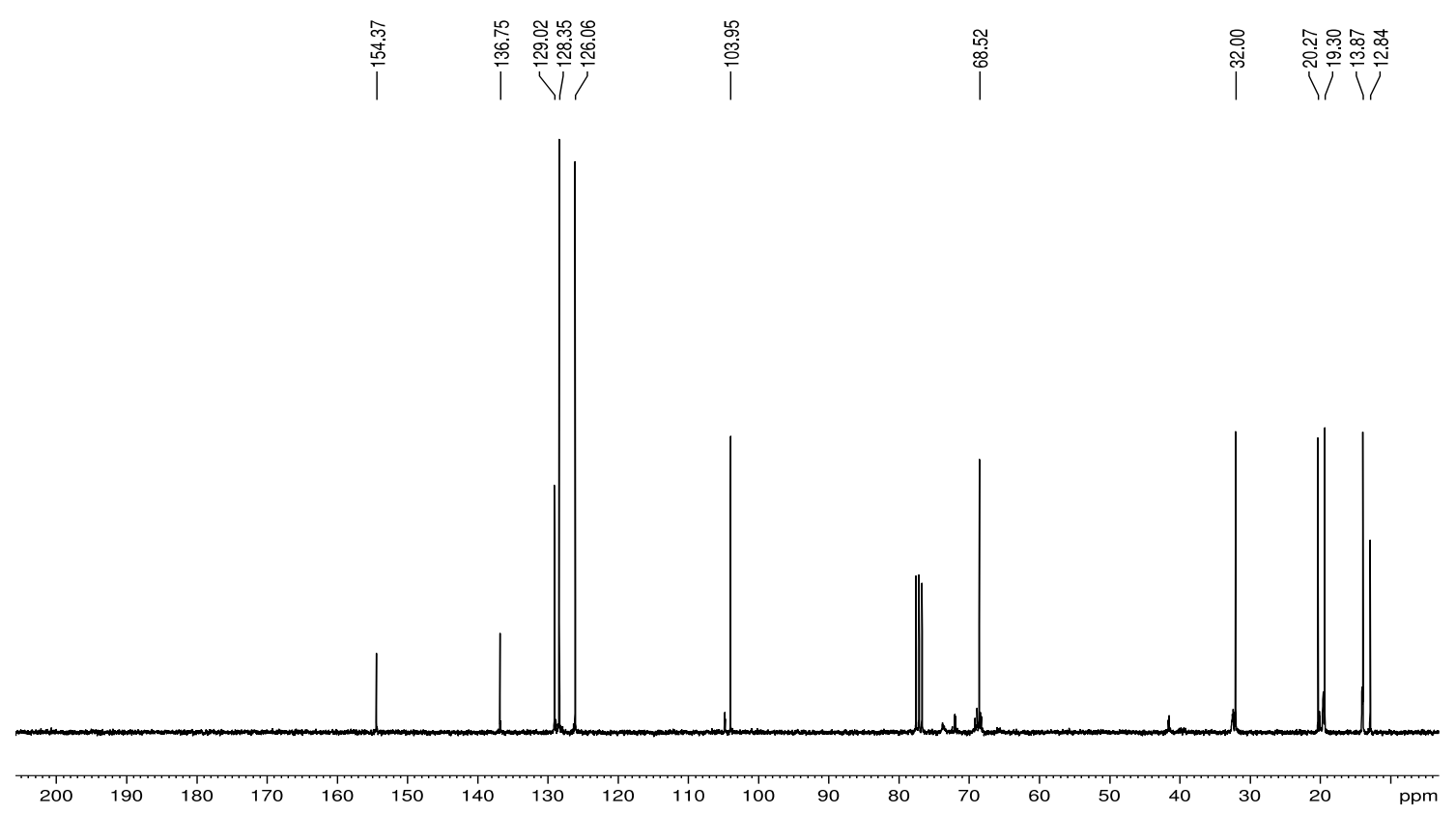


$\underline{{ }^{1} \mathrm{H} N M R}(300 \mathrm{MHz})$ Spectrum of 7aa ( $\boldsymbol{E}$ isomer) in $\mathrm{CDCl}_{3}$<smiles>C/C(=N\OC(C)OCC(C)C)c1ccccc1</smiles>

\section{눈}

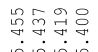
पें

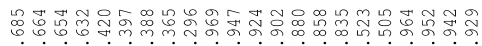

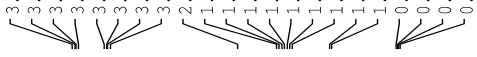
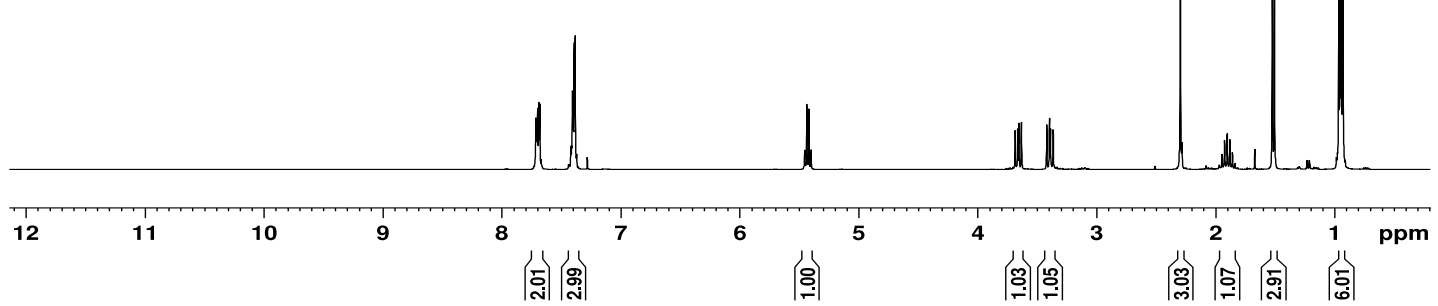

${ }^{13} \mathrm{C}\left\{{ }^{1} \mathrm{H}\right\}$ NMR (75.5 MHz) Spectrum of 7aa ( $\boldsymbol{E}$ isomer) in $\mathrm{CDCl}_{3}$

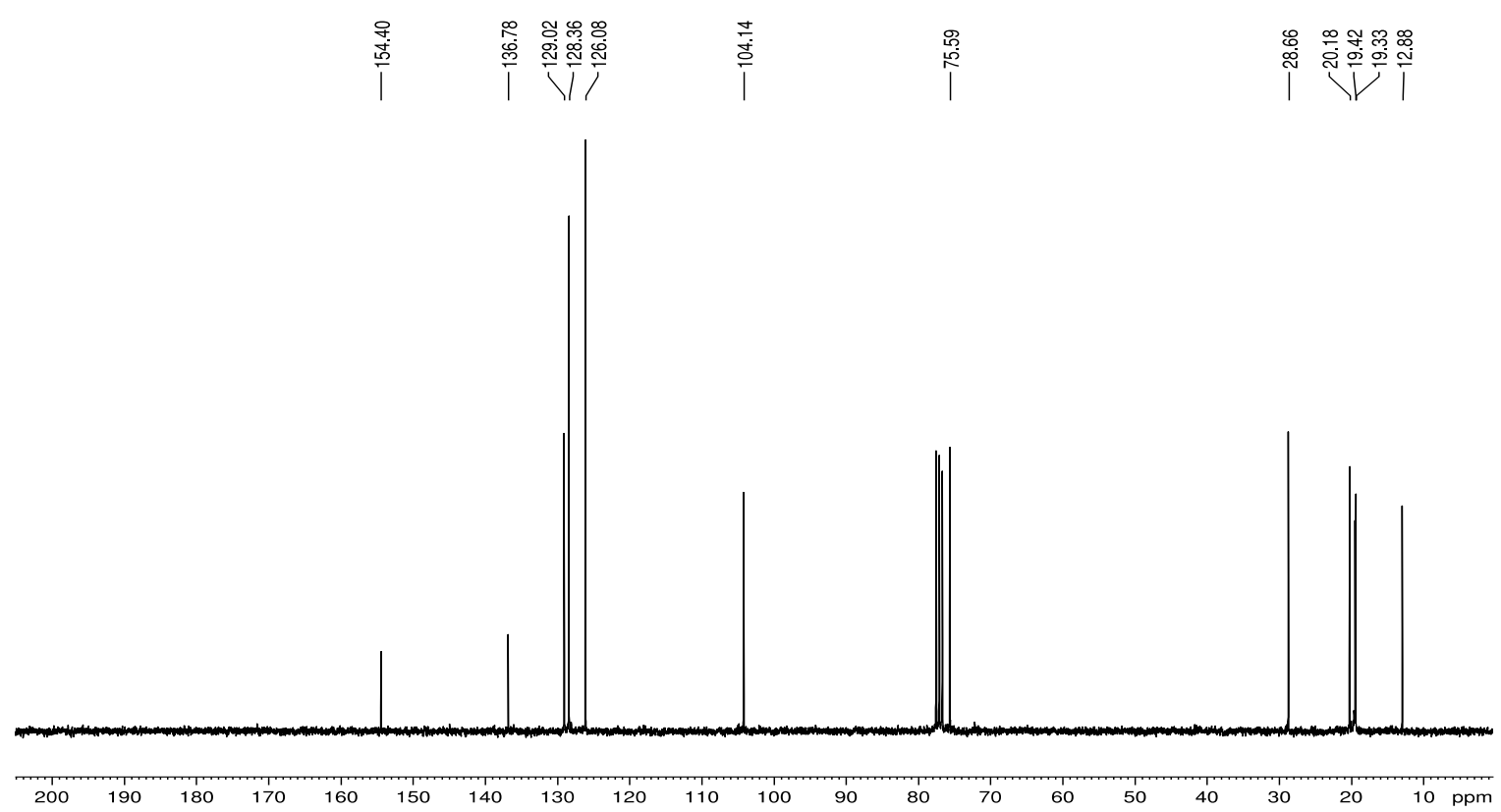




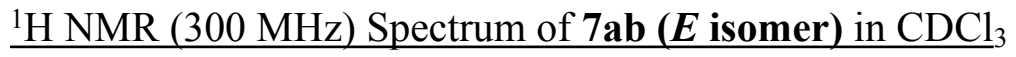
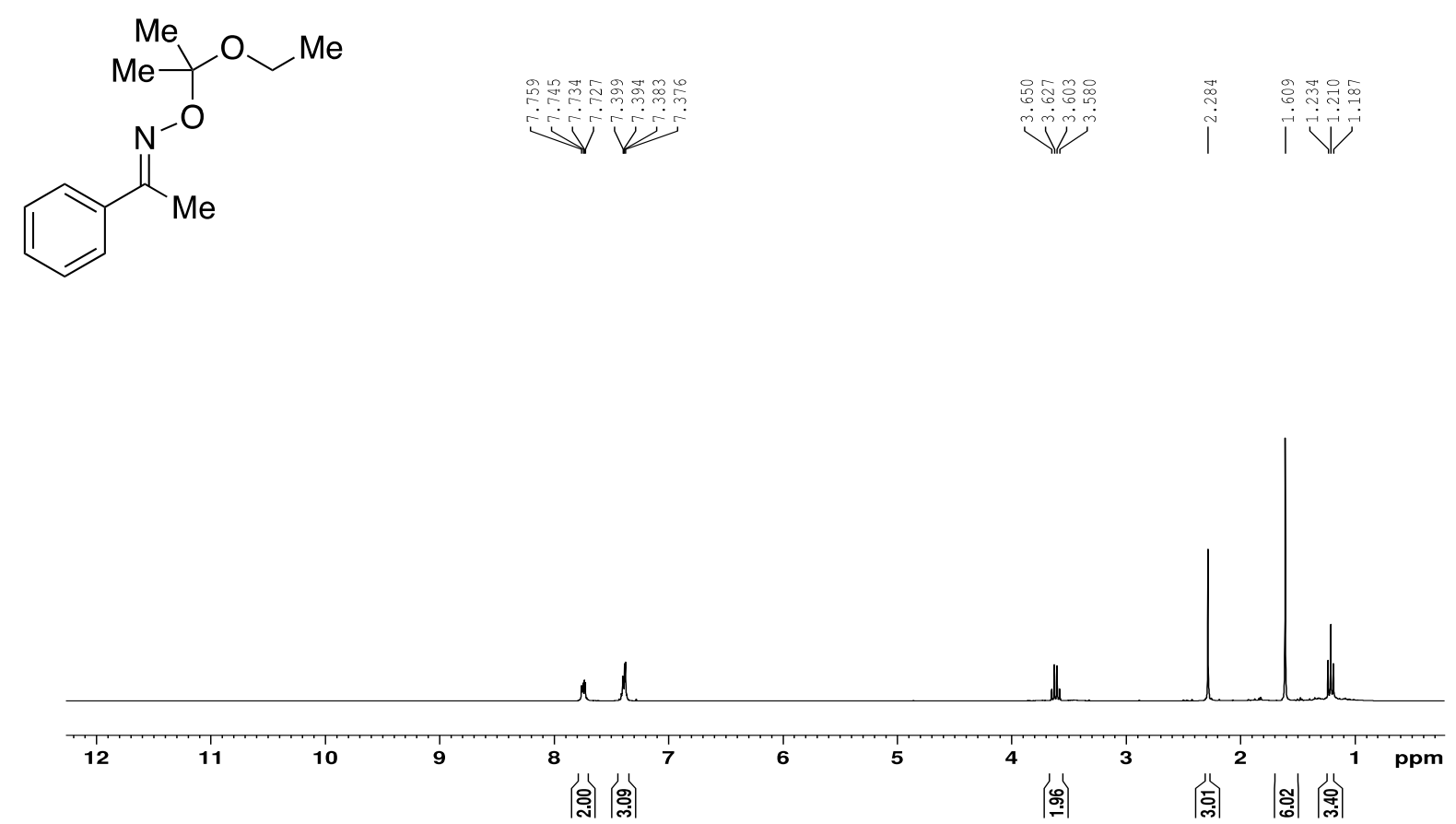

${ }^{13} \mathrm{C}\left\{{ }^{1} \mathrm{H}\right\}$ NMR (75.5 MHz) Spectrum of $7 \mathbf{a b}\left(\boldsymbol{E}\right.$ isomer) in $\mathrm{CDCl}_{3}$
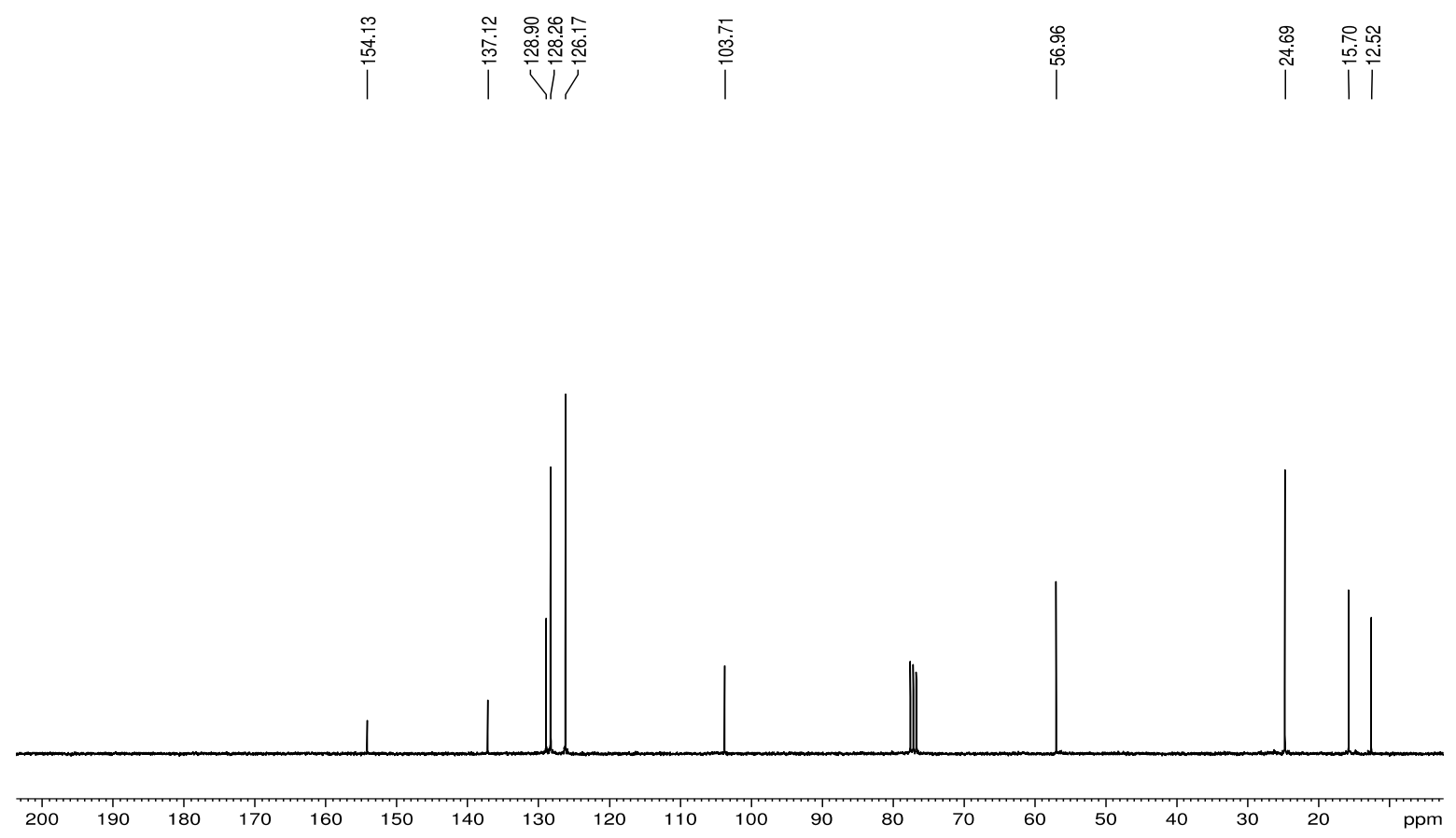


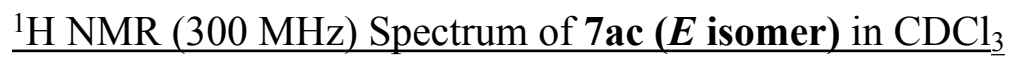
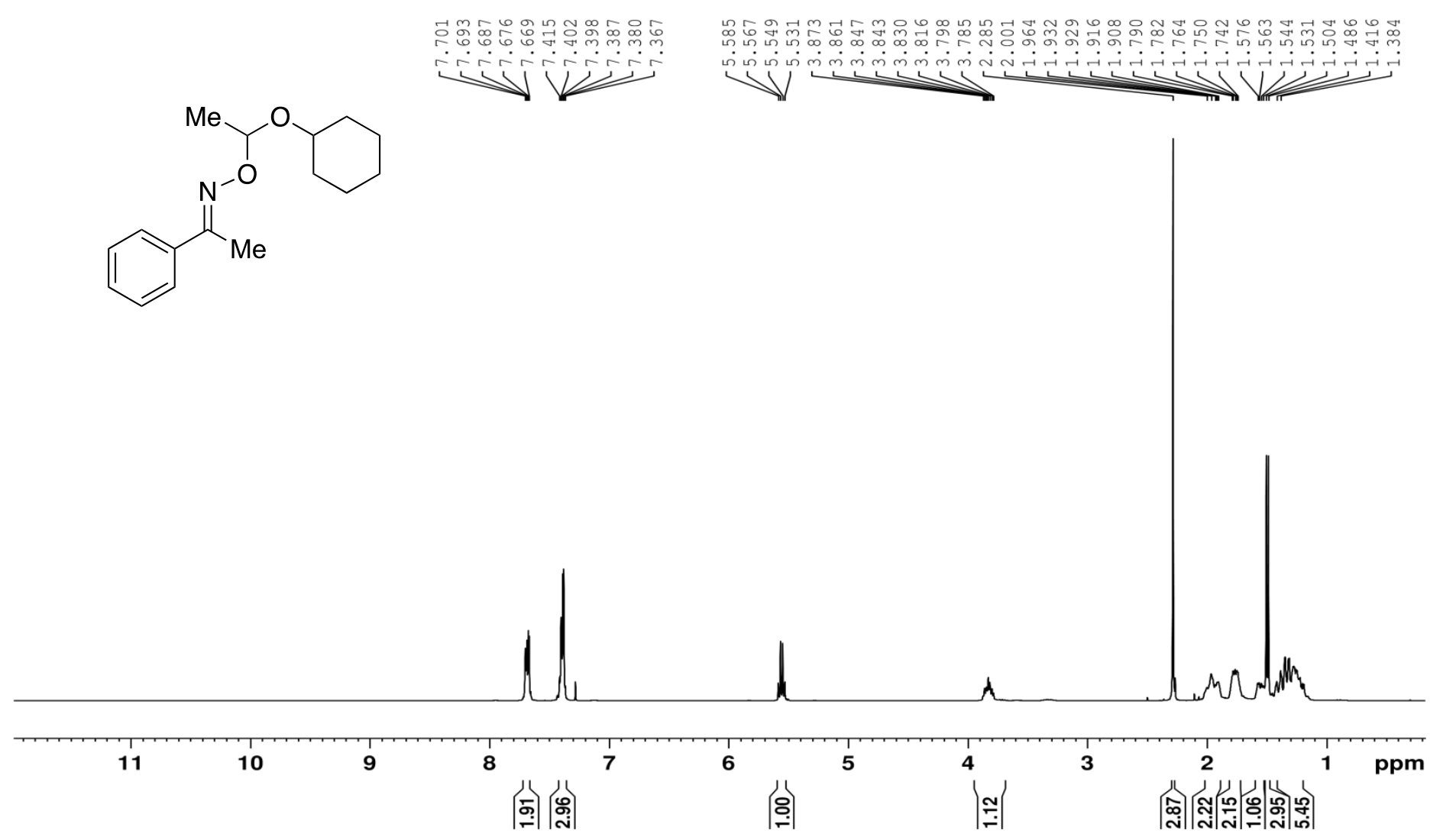

$\left.{ }^{13} \mathrm{C}^{1}{ }^{1} \mathrm{H}\right\}$ NMR (75.5 MHz) Spectrum of 7ac ( $\boldsymbol{E}$ isomer) in $\mathrm{CDCl}_{3}$

| |

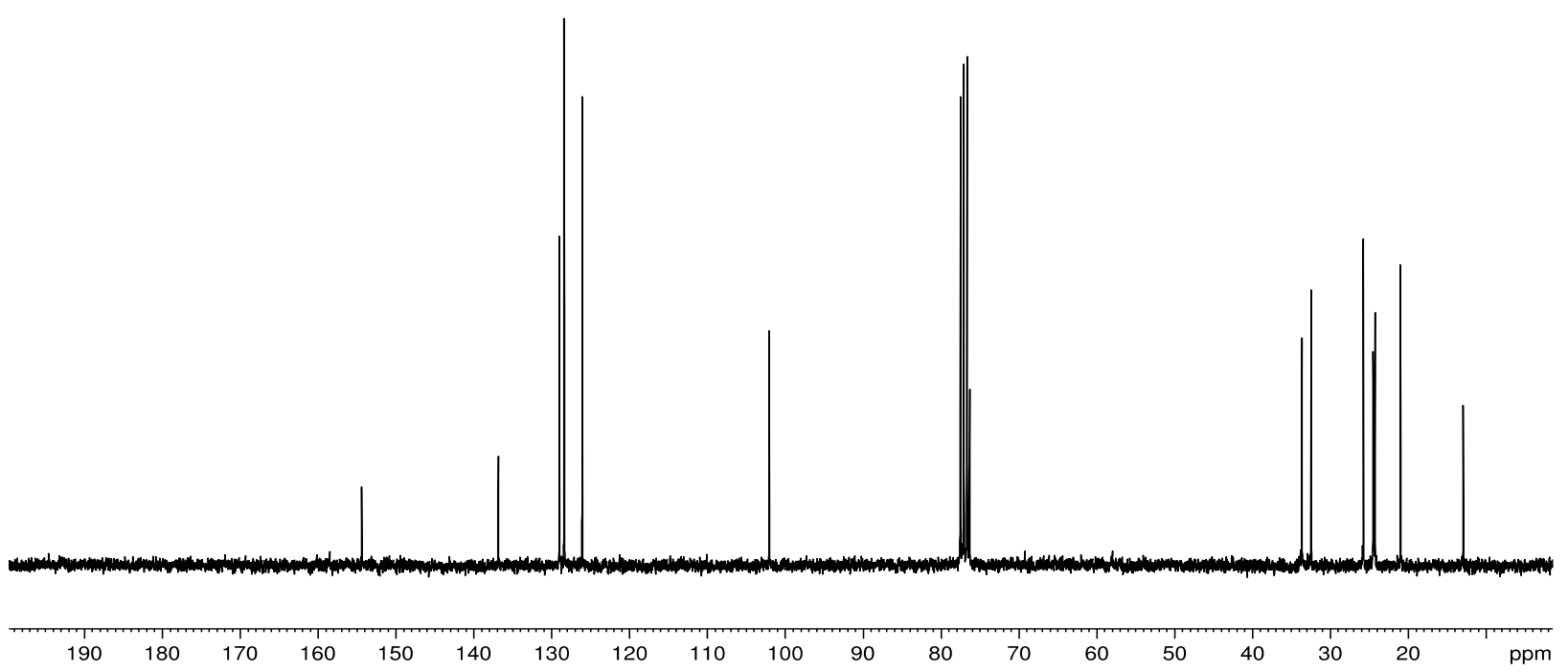


$\underline{{ }^{1} \mathrm{H} N M R}(300 \mathrm{MHz})$ Spectrum of $7 \mathbf{a d}\left(\boldsymbol{E}\right.$ isomer) in $\mathrm{CDCl}_{3}$

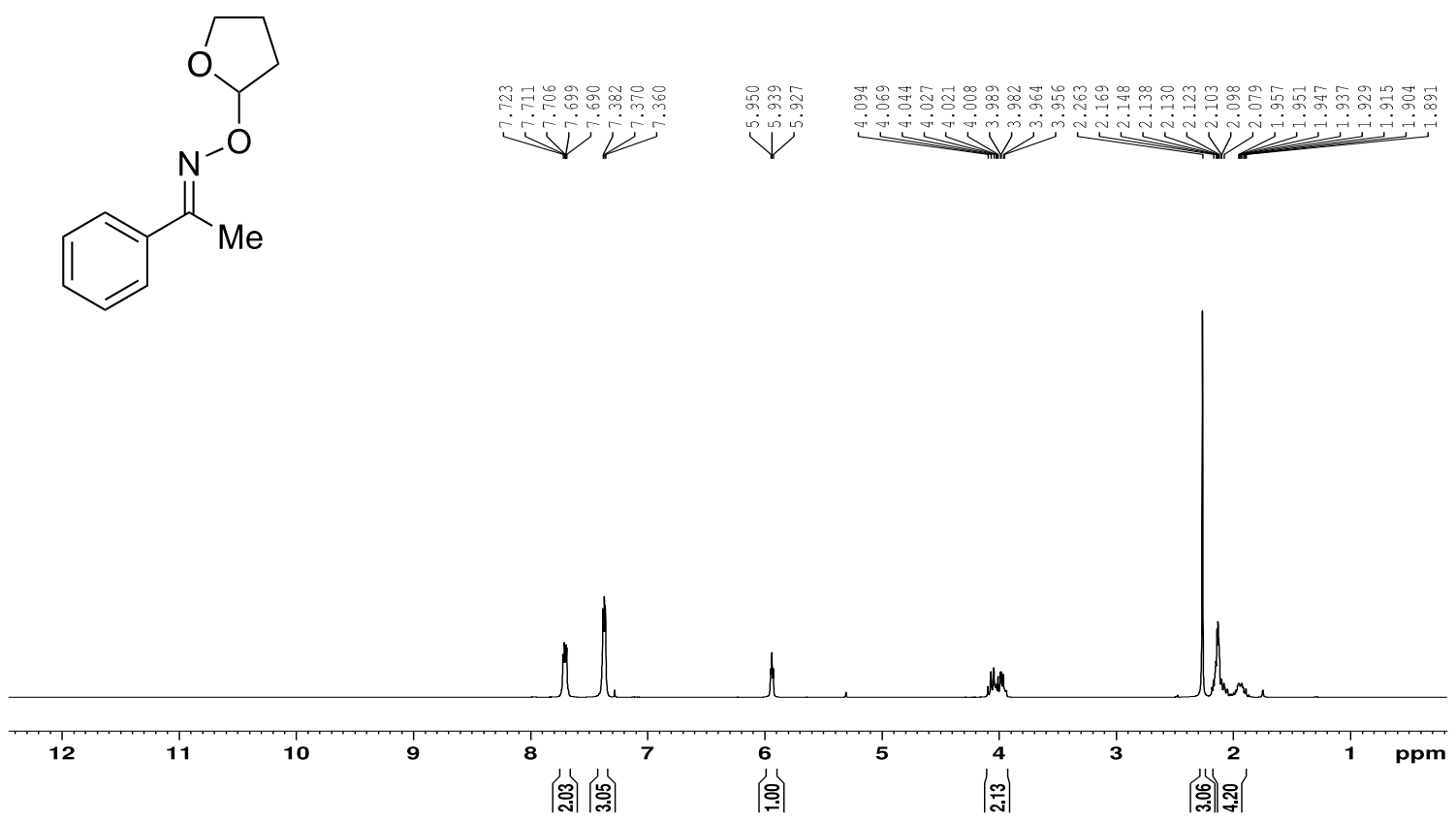

${ }^{13} \mathrm{C}\left\{{ }^{1} \mathrm{H}\right\}$ NMR (75.5 MHz) Spectrum of 7ad ( $\boldsymbol{E}$ isomer) in $\mathrm{CDCl}_{3}$

|

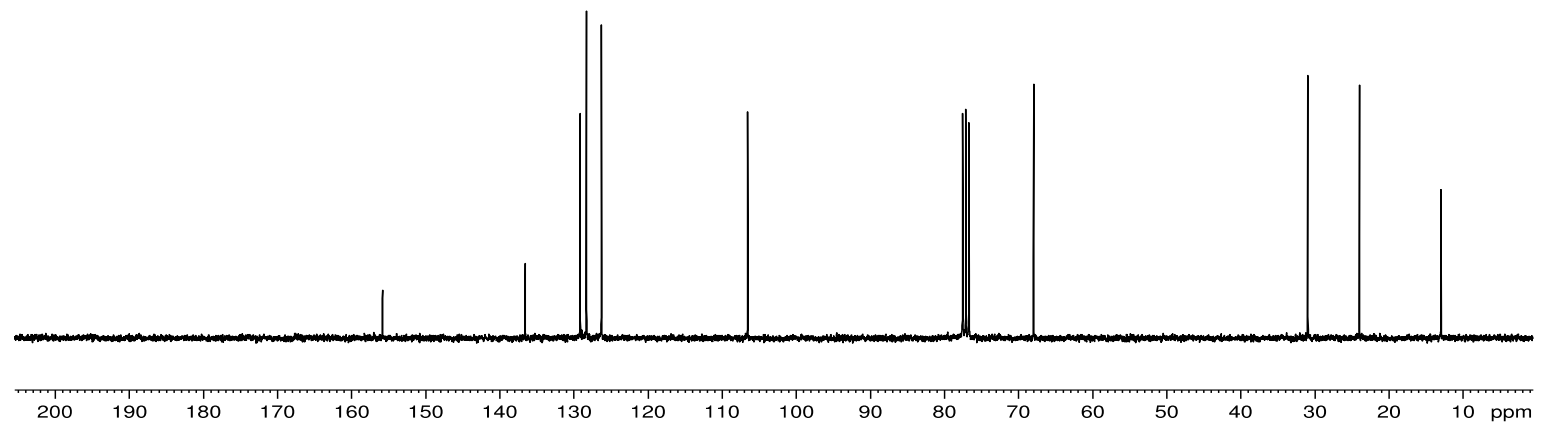


${ }^{1} \mathrm{H}$ NMR (300 MHz) Spectrum of 7ae ( $\boldsymbol{E}$ isomer) in $\mathrm{CDCl}_{3}$

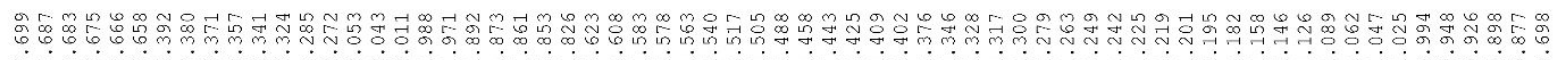
(r.

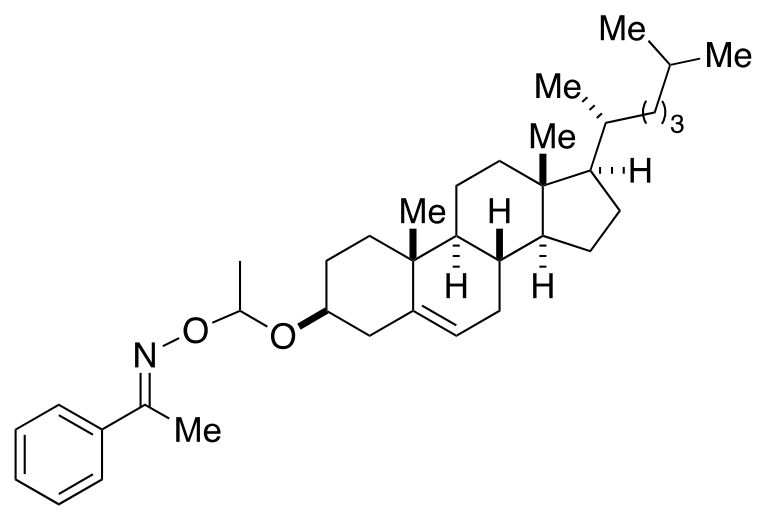
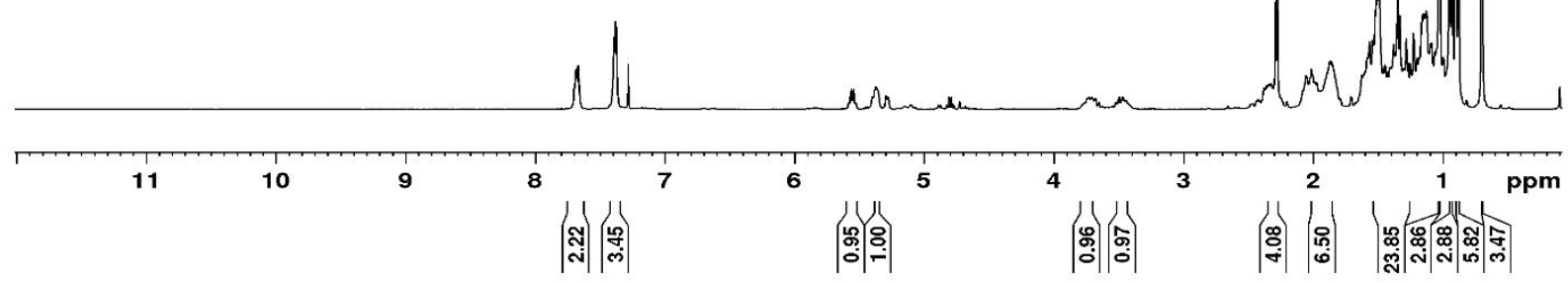

${ }^{13} \mathrm{C}\left\{{ }^{1} \mathrm{H}\right\} \mathrm{NMR}(75.5 \mathrm{MHz})$ Spectrum of $7 \mathbf{a e}\left(\boldsymbol{E}\right.$ isomer) in $\mathrm{CDCl}_{3}$

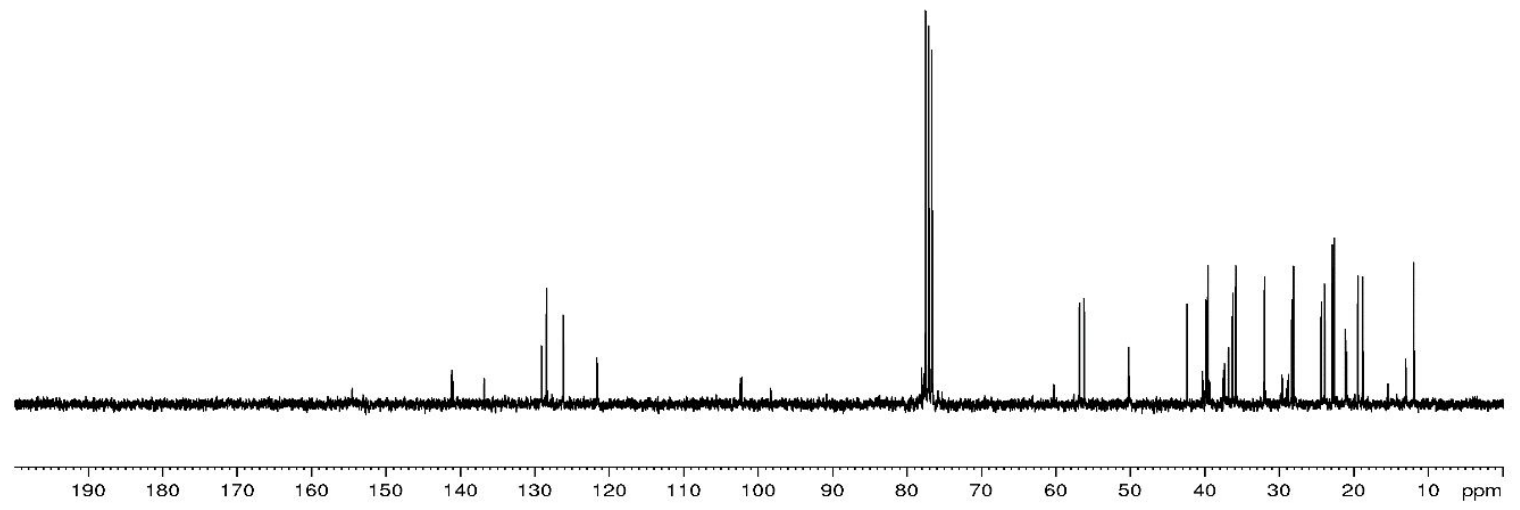


$\underline{{ }^{1} \mathrm{H} \text { NMR (300 MHz) Spectrum of a 1:3 Z/E mixture of 7af in } \mathrm{CDCl}_{3}}$

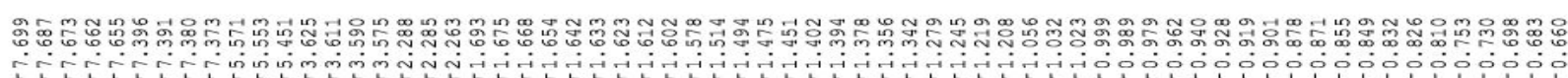

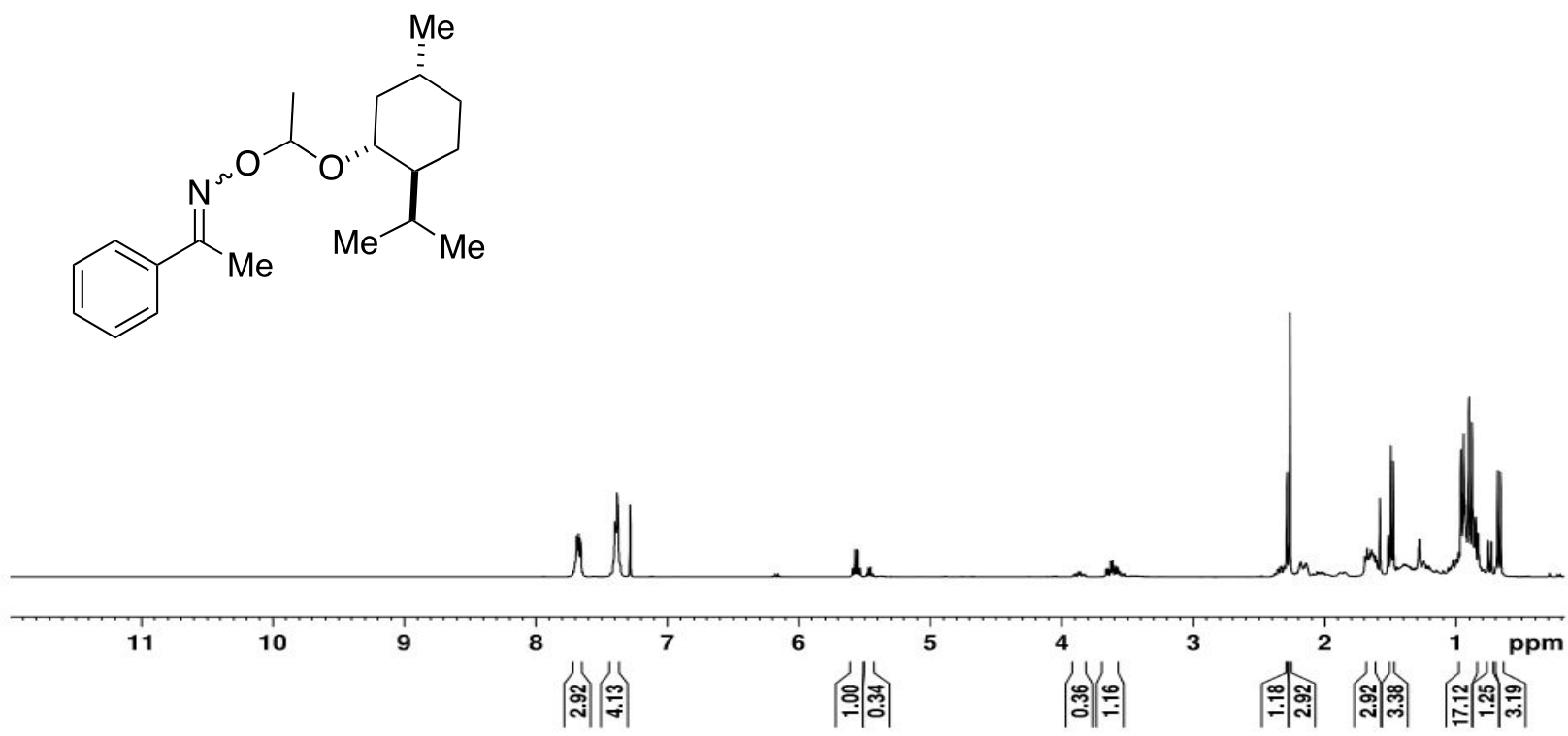

${ }^{13} \mathrm{C}\left\{{ }^{1} \mathrm{H}\right\}$ NMR (75.5 MHz) Spectrum of a 1:3 Z/E mixture of 7af in $\mathrm{CDCl}_{\underline{3}}$

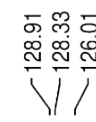

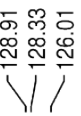

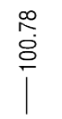

œ

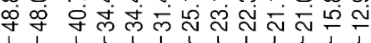

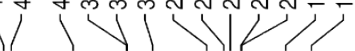

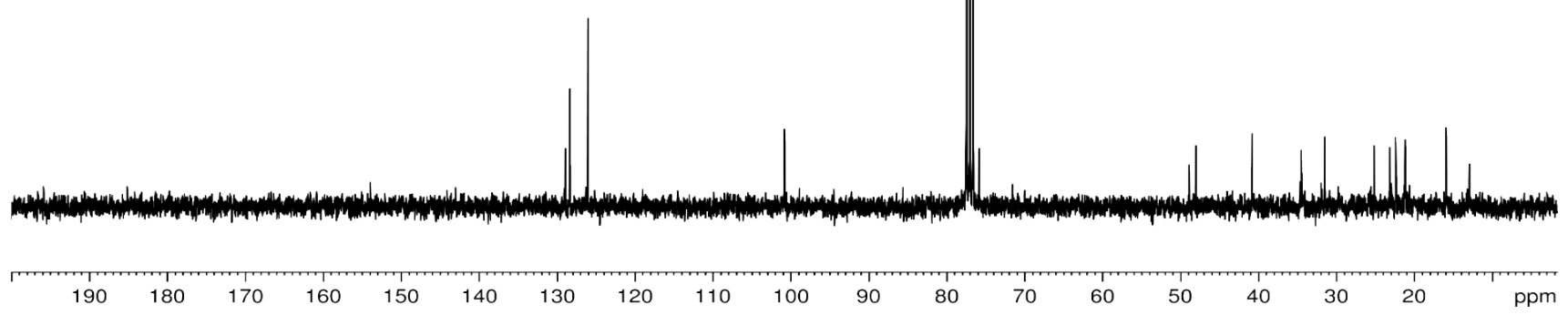


$\underline{{ }^{1} \mathrm{H} N M R}(300 \mathrm{MHz})$ Spectrum of $7 \mathbf{a g}\left(\boldsymbol{E}\right.$ isomer) in $\mathrm{CDCl}_{3}$

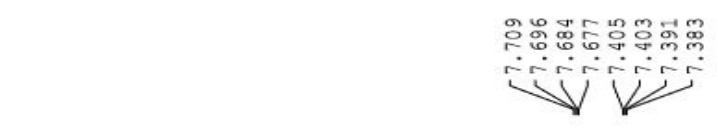<smiles>CC(=NOC(C)OCCCCCCCl)c1ccccc1</smiles>

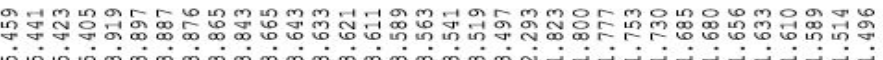
$\mathrm{V}$

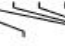


$\underline{{ }^{1} \mathrm{H} N M R}(300 \mathrm{MHz})$ Spectrum of 7ah ( $\boldsymbol{E}$ isomer) in $\mathrm{CDCl}_{3}$

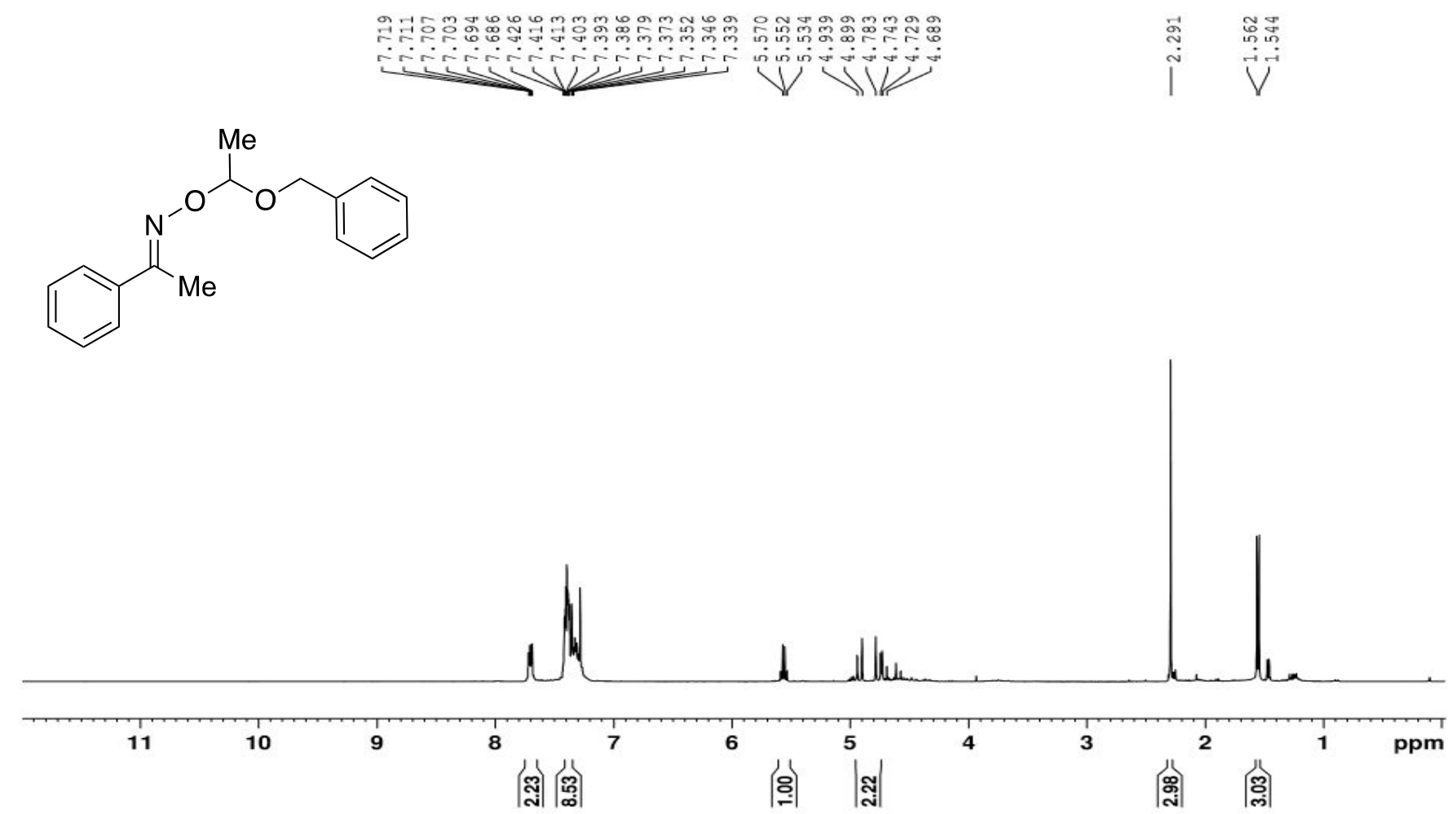

$\underline{{ }^{13} \mathrm{C}\left\{{ }^{1} \mathrm{H}\right\} \text { NMR (75.5 MHz) Spectrum of } 7 \mathbf{a h}\left(\boldsymbol{E} \text { isomer) in } \mathrm{CDCl}_{3}\right.}$
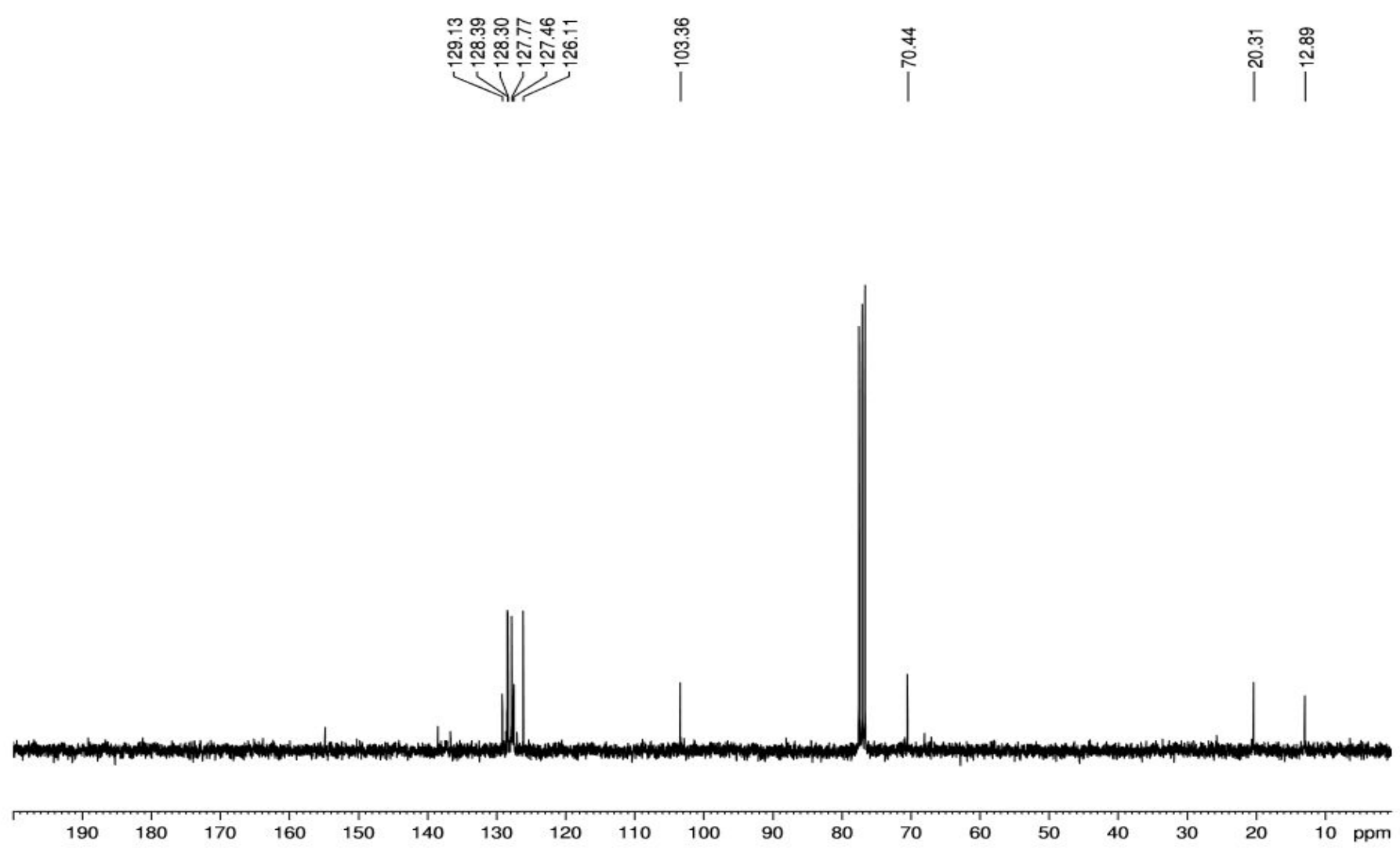
$\underline{{ }^{1} \mathrm{H} \text { NMR (300 MHz) Spectrum of } 8 \text { in } \mathrm{CDCl}_{3}}$
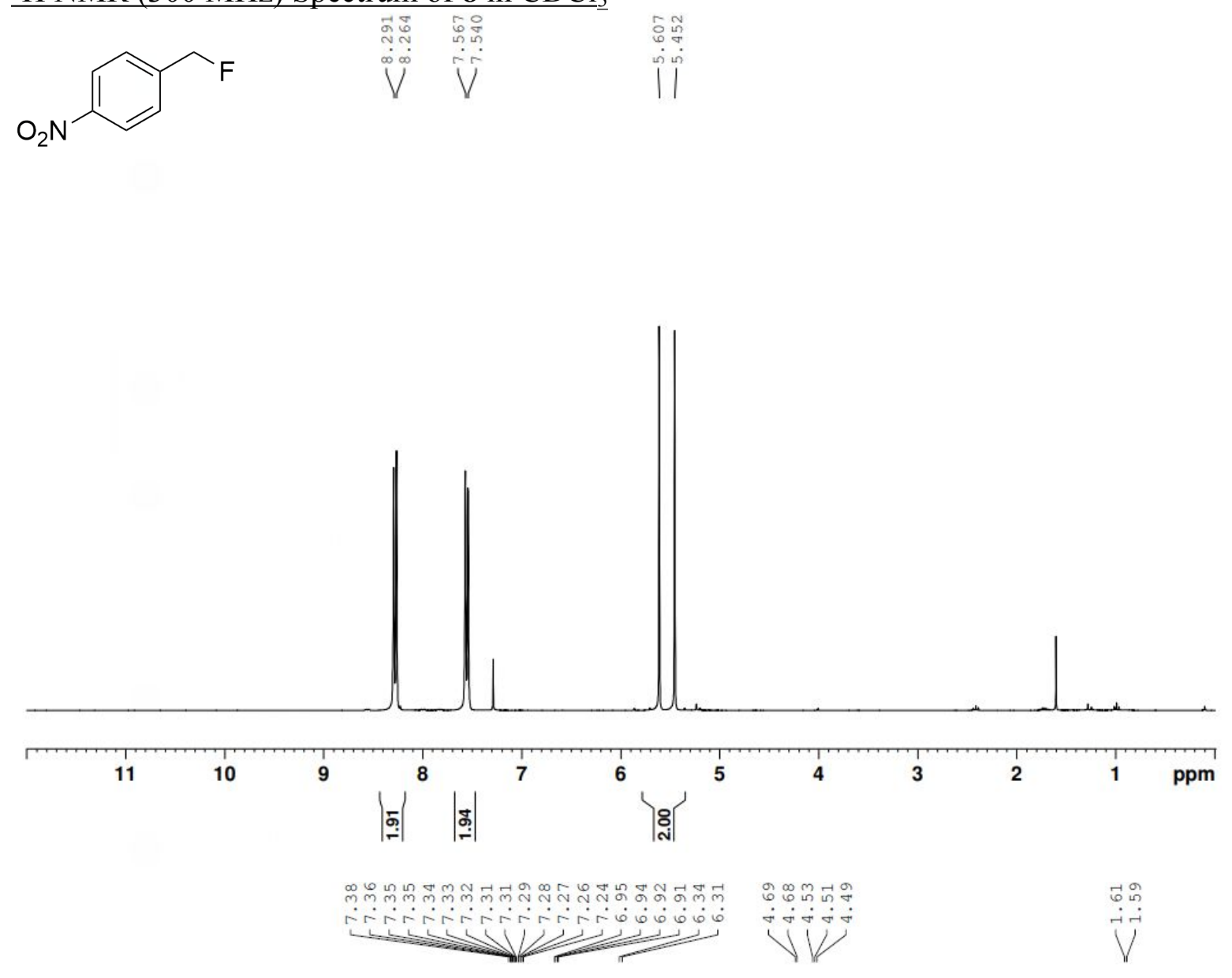

${ }^{1} \mathrm{H} \mathrm{NMR}(400 \mathrm{MHz})$ Spectrum of 9 in $\mathrm{CDCl}_{3}$<smiles>CC(Nc1ccc(Cl)cc1Cl)c1ccccc1</smiles>

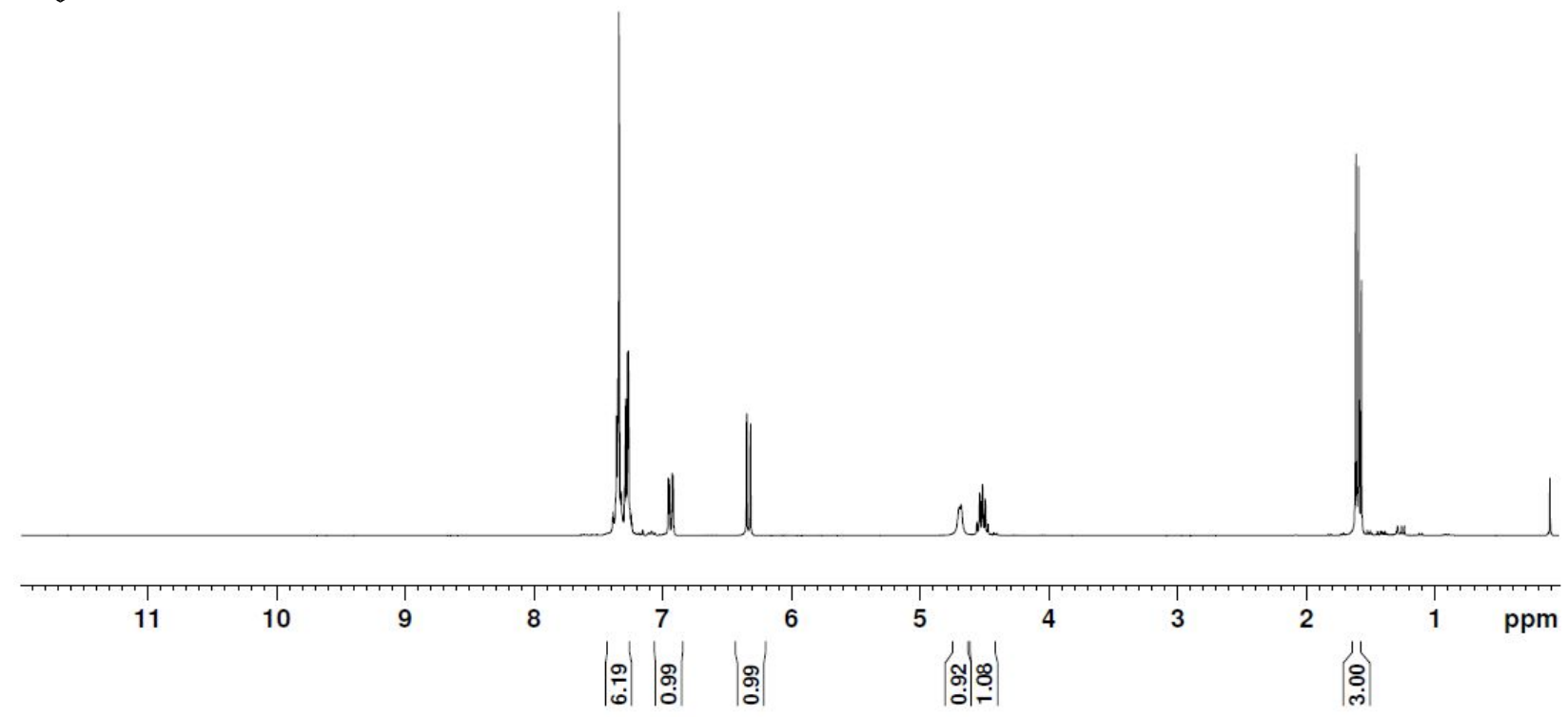


$\underline{{ }^{1} \mathrm{H} \text { NMR (400 MHz) Spectrum of } \mathbf{1 0} \text { in } \mathrm{CDCl}_{3}}$

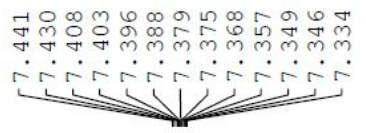

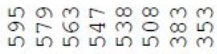

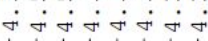

萬尔

$\therefore \sin 5$

Wh

iे<smiles>CC(OCc1ccccc1)c1ccccc1</smiles>

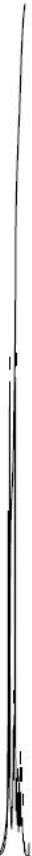
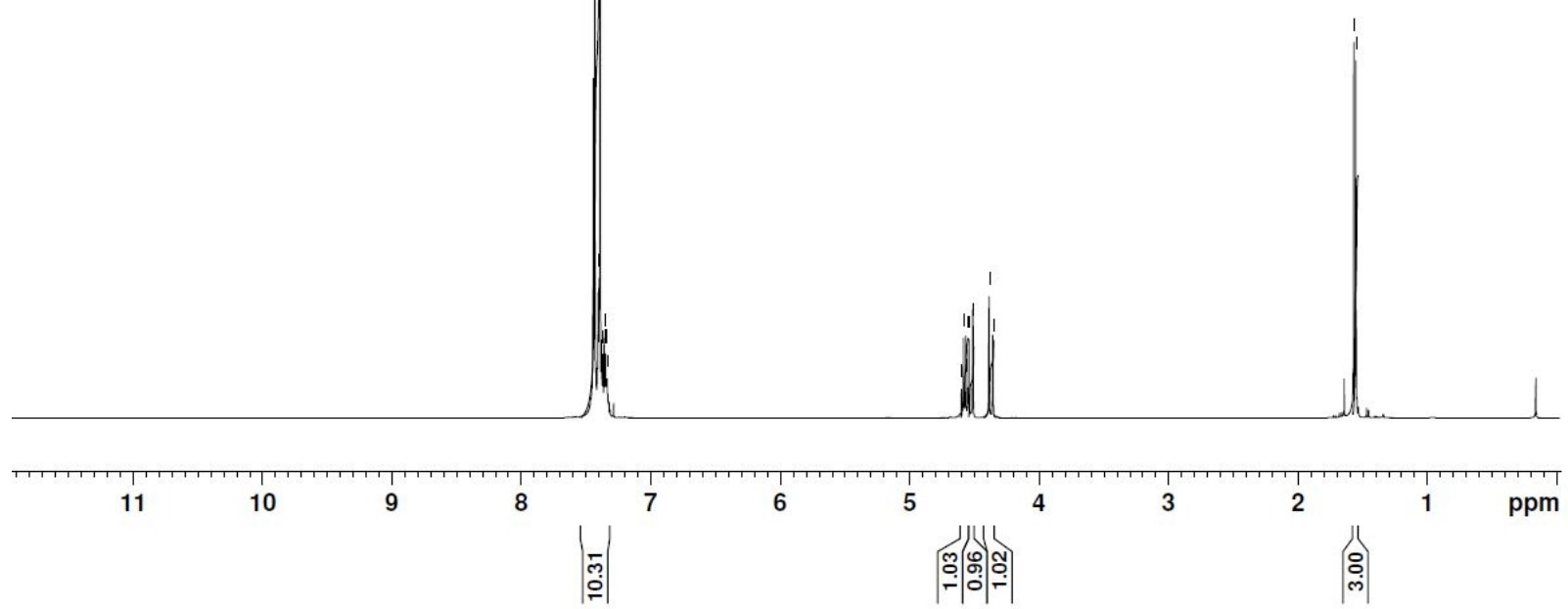

${ }^{1} \mathrm{H}$ NMR (300 MHz) Spectrum of 11 in $\mathrm{CDCl}_{3}$

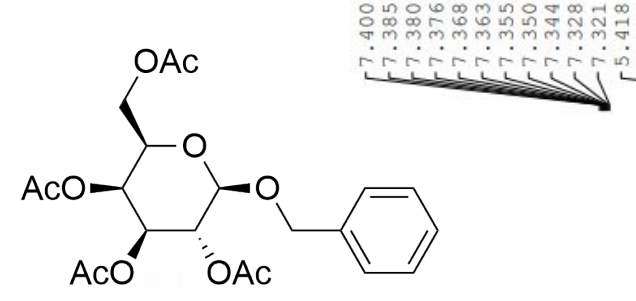




\section{X-ray crystallographic analysis}

Single-crystals of $\mathbf{4} \mathbf{e} \cdot \mathbf{B F}_{4}{ }^{-}$were mounted on a cryoloop with paratone oil and examined on an APEX II CCD X-ray diffractometer using graphite-monochromated Mo-K $\alpha$ radiation $(\lambda=0.71073$ $\AA$ ). An Oxford Cryostream cooler was used to maintain cryogenic temperatures. Data were collected using the APEX-II software, ${ }^{24}$ integrated using SAINT ${ }^{25}$ and corrected for absorption using the multi-scan approach (SADABS) ${ }^{26}$ Final cell constants were determined from full least squares refinement of all observed reflections. The structures were solved using intrinsic phasing (SHELXT). ${ }^{27}$ All structures were refined with full squares refinement on $\mathrm{F}^{2}$ using the SHELXTL software. ${ }^{28}$ All hydrogen atoms were added at calculated positions and refined isotropically with a riding model. Both $\mathrm{CF}_{3}$ groups were highly disordered and successfully modelled over two and three positions, respectively. Disordered solvent was removed from the crystallographic model using PLATON/SQUEEZE. ${ }^{29}$ A thermal ellipsoid plot of the complex with the appropriate atomic labelling scheme is given in Figure S5. A summary of the experimental crystallographic data for the complex is presented in Table S6. Select bond lengths and angles are provided in Table S7, and a summary of the H-bonding interactions are presented in Table S8.

Table S6. Select Crystallographic Parameters for $\mathbf{4 e} \cdot \mathbf{B F}_{4}^{-}$.

\begin{tabular}{|c|c|}
\hline Chemical formula & $\mathrm{C}_{27} \mathrm{H}_{33} \mathrm{BF}_{10} \mathrm{~N}_{4} \mathrm{O}_{2}$ \\
\hline$M_{\mathrm{r}}$ & 646.38 \\
\hline Crystal system, space group & Orthorhombic, $\mathrm{Pbca}$ \\
\hline Temperature (K) & 150 \\
\hline$a, b, c(\AA)$ & $7.9774(7), 29.030(2), 30.322(3)$ \\
\hline$V\left(\AA^{3}\right)$ & $7022.1(10)$ \\
\hline$Z$ & 8 \\
\hline Radiation type & Mo $K \alpha$ \\
\hline$\mu\left(\mathrm{mm}^{-1}\right)$ & 0.11 \\
\hline Crystal size (mm) & $0.2 \times 0.05 \times 0.05$ \\
\hline \multicolumn{2}{|l|}{ Data collection } \\
\hline Diffractometer & Bruker $A P E X$-II CCD \\
\hline Absorption correction & $\begin{array}{l}\text { Multi-scan } \\
\text { Bruker } S A D A B S\end{array}$ \\
\hline$T_{\min }, T_{\max }$ & $0.435,0.786$ \\
\hline $\begin{array}{l}\text { No. of measured, independent and } \\
\text { observed }[I>2 \sigma(I)] \text { reflections }\end{array}$ & $28152,6853,4548$ \\
\hline$R_{\text {int }}$ & 0.085 \\
\hline$(\sin \theta / \lambda)_{\max }\left(\AA^{-1}\right)$ & 0.628 \\
\hline \multicolumn{2}{|l|}{ Refinement } \\
\hline$R\left[F^{2}>2 \sigma\left(F^{2}\right)\right], w R\left(F^{2}\right), S$ & $0.128,0.337,1.06$ \\
\hline No. of reflections & 6853 \\
\hline No. of parameters & 413 \\
\hline No. of restraints & 31 \\
\hline H-atom treatment & H-atom parameters constrained \\
\hline
\end{tabular}




$$
w=1 /\left[\sigma^{2}\left(F_{\mathrm{o}}^{2}\right)+(0.0963 P)^{2}+55.5373 P\right]
$$

where $P=\left(F_{\mathrm{o}}^{2}+2 F_{\mathrm{c}}^{2}\right) / 3$

$\underline{\Delta \rho_{\max }, \Delta \rho_{\min }\left(\mathrm{e} \AA^{-3}\right)}$

$0.69,-0.46$

Computer programs: Bruker APEX2, ${ }^{24}$ Bruker SAINT, ${ }^{25}$ SHELXT (Sheldrick 2015), ${ }^{27}$

SHELXL2014 (Sheldrick 2014), ${ }^{28}$ Bruker SHELXTL. ${ }^{30}$

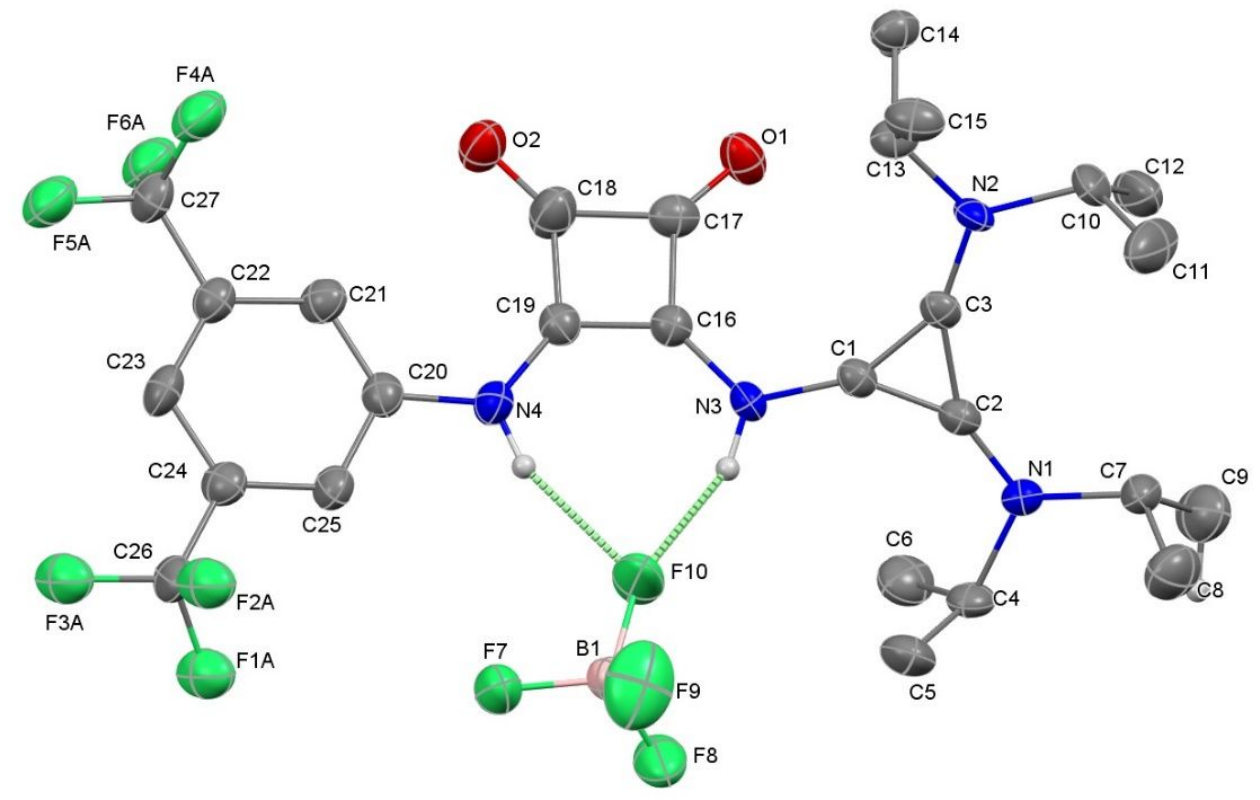

Figure S5. ORTEP plot of the molecular structure of $4 \mathbf{e} \cdot \mathbf{B F}_{4}{ }^{-}$with the appropriate atomic labelling scheme. Thermal ellipsoids are plotted at $50 \%$. For clarity only select $\mathrm{H}$ atoms are shown, and only one orientation of the disordered $\mathrm{CF}_{3}$ groups is shown. Short contacts from $\mathrm{F}(10)$ of the $\mathrm{BF}_{4}{ }^{-}$counterion to $\mathrm{H}(41)$ and $\mathrm{H}(31)$ are shown as red dashed lines.

Table S7. Selected geometric parameters for $\mathbf{4 e} \cdot \mathbf{B F}_{4}{ }^{-}\left(\AA,{ }^{\circ}\right)$

\begin{tabular}{cccc} 
Bond & Length $(\AA)$ & Bond & Length $(\AA)$ \\
\hline F1A-C26 & $1.338(12)$ & N3-C16 & $1.348(7)$ \\
F2A-C26 & $1.386(11)$ & N3-C1 & $1.375(7)$ \\
F3A-C26 & $1.342(10)$ & N4-C19 & $1.360(8)$ \\
F1B-C26 & $1.327(12)$ & N4-C20 & $1.405(8)$ \\
F2B-C26 & $1.381(10)$ & $\mathrm{C} 1-\mathrm{C} 2$ & $1.363(8)$ \\
F3B-C26 & $1.318(10)$ & $\mathrm{C} 1-\mathrm{C} 3$ & $1.369(8)$ \\
F4A-C27 & $1.345(11)$ & $\mathrm{C} 2-\mathrm{C} 3$ & $1.421(8)$ \\
F5A-C27 & $1.327(10)$ & $\mathrm{C} 4-\mathrm{C} 6$ & $1.511(10)$ \\
F6A-C27 & $1.346(11)$ & $\mathrm{C} 4-\mathrm{C} 5$ & $1.519(10)$ \\
F4B-C27 & $1.311(11)$ & $\mathrm{C} 7-\mathrm{C} 9$ & $1.507(10)$ \\
F5B-C27 & $1.313(12)$ & $\mathrm{C} 7-\mathrm{C} 8$ & $1.528(10)$ \\
F6B-C27 & $1.383(12)$ & $\mathrm{C} 10-\mathrm{C} 12$ & $1.494(11)$ \\
\hline
\end{tabular}




\begin{tabular}{|c|c|c|c|}
\hline $\mathrm{F} 4 \mathrm{C}-\mathrm{C} 27$ & $1.343(15)$ & $\mathrm{C} 10-\mathrm{C} 11$ & $1.533(11)$ \\
\hline $\mathrm{F} 5 \mathrm{C}-\mathrm{C} 27$ & $1.390(15)$ & $\mathrm{C} 13-\mathrm{C} 14$ & $1.526(8)$ \\
\hline $\mathrm{F} 6 \mathrm{C}-\mathrm{C} 27$ & $1.334(15)$ & $\mathrm{C} 13-\mathrm{C} 15$ & $1.528(9)$ \\
\hline F7-B1 & $1.398(9)$ & $\mathrm{C} 16-\mathrm{C} 19$ & $1.386(8)$ \\
\hline $\mathrm{F} 8-\mathrm{B} 1$ & $1.371(9)$ & $\mathrm{C} 16-\mathrm{C} 17$ & $1.476(8)$ \\
\hline F9-B1 & $1.343(9)$ & $\mathrm{C} 17-\mathrm{C} 18$ & $1.514(9)$ \\
\hline $\mathrm{F} 10-\mathrm{B} 1$ & $1.391(8)$ & $\mathrm{C} 18-\mathrm{C} 19$ & $1.479(9)$ \\
\hline $\mathrm{O} 1-\mathrm{C} 17$ & $1.209(8)$ & $\mathrm{C} 20-\mathrm{C} 21$ & $1.386(9)$ \\
\hline $\mathrm{O} 2-\mathrm{C} 18$ & $1.221(8)$ & $\mathrm{C} 20-\mathrm{C} 25$ & $1.414(9)$ \\
\hline $\mathrm{N} 1-\mathrm{C} 2$ & $1.302(7)$ & $\mathrm{C} 21-\mathrm{C} 22$ & $1.390(9)$ \\
\hline $\mathrm{N} 1-\mathrm{C} 7$ & $1.484(8)$ & $\mathrm{C} 22-\mathrm{C} 23$ & $1.383(9)$ \\
\hline $\mathrm{N} 1-\mathrm{C} 4$ & $1.496(7)$ & $\mathrm{C} 22-\mathrm{C} 27$ & $1.494(9)$ \\
\hline $\mathrm{N} 2-\mathrm{C} 3$ & $1.323(7)$ & $\mathrm{C} 23-\mathrm{C} 24$ & $1.388(9)$ \\
\hline $\mathrm{N} 2-\mathrm{C} 13$ & $1.472(8)$ & $\mathrm{C} 24-\mathrm{C} 25$ & $1.389(9)$ \\
\hline $\mathrm{N} 2-\mathrm{C} 10$ & $1.497(8)$ & $\mathrm{C} 24-\mathrm{C} 26$ & $1.488(9)$ \\
\hline Bond & Angle $\left(^{\circ}\right)$ & Bond & Angle ( $\left.{ }^{\circ}\right)$ \\
\hline $\mathrm{F} 9-\mathrm{B} 1-\mathrm{F} 8$ & $109.2(6)$ & $\mathrm{N} 4-\mathrm{C} 19-\mathrm{C} 18$ & $139.4(6)$ \\
\hline $\mathrm{F} 9-\mathrm{B} 1-\mathrm{F} 10$ & $110.7(7)$ & $\mathrm{C} 16-\mathrm{C} 19-\mathrm{C} 18$ & $91.8(5)$ \\
\hline $\mathrm{F} 8-\mathrm{B} 1-\mathrm{F} 10$ & $110.4(6)$ & $\mathrm{C} 21-\mathrm{C} 20-\mathrm{N} 4$ & $123.3(6)$ \\
\hline $\mathrm{F} 9-\mathrm{B} 1-\mathrm{F} 7$ & $110.1(6)$ & $\mathrm{C} 21-\mathrm{C} 20-\mathrm{C} 25$ & $120.3(6)$ \\
\hline $\mathrm{F} 8-\mathrm{B} 1-\mathrm{F} 7$ & $109.3(6)$ & $\mathrm{N} 4-\mathrm{C} 20-\mathrm{C} 25$ & $116.3(6)$ \\
\hline $\mathrm{F} 10-\mathrm{B} 1-\mathrm{F} 7$ & $107.0(6)$ & $\mathrm{C} 20-\mathrm{C} 21-\mathrm{C} 22$ & $119.1(6)$ \\
\hline $\mathrm{C} 2-\mathrm{N} 1-\mathrm{C} 7$ & $121.9(5)$ & $\mathrm{C} 23-\mathrm{C} 22-\mathrm{C} 21$ & $121.8(6)$ \\
\hline $\mathrm{C} 2-\mathrm{N} 1-\mathrm{C} 4$ & $118.4(5)$ & $\mathrm{C} 23-\mathrm{C} 22-\mathrm{C} 27$ & $119.5(5)$ \\
\hline $\mathrm{C} 7-\mathrm{N} 1-\mathrm{C} 4$ & $119.4(5)$ & $\mathrm{C} 21-\mathrm{C} 22-\mathrm{C} 27$ & $118.6(5)$ \\
\hline $\mathrm{C} 3-\mathrm{N} 2-\mathrm{C} 13$ & $119.5(5)$ & $\mathrm{C} 22-\mathrm{C} 23-\mathrm{C} 24$ & $118.8(6)$ \\
\hline $\mathrm{C} 3-\mathrm{N} 2-\mathrm{C} 10$ & $121.0(5)$ & $\mathrm{C} 25-\mathrm{C} 24-\mathrm{C} 23$ & $121.2(6)$ \\
\hline $\mathrm{C} 13-\mathrm{N} 2-\mathrm{C} 10$ & $119.5(5)$ & $\mathrm{C} 25-\mathrm{C} 24-\mathrm{C} 26$ & $119.1(6)$ \\
\hline $\mathrm{C} 16-\mathrm{N} 3-\mathrm{C} 1$ & $123.3(5)$ & $\mathrm{C} 23-\mathrm{C} 24-\mathrm{C} 26$ & $119.5(6)$ \\
\hline $\mathrm{C} 19-\mathrm{N} 4-\mathrm{C} 20$ & $127.6(5)$ & $\mathrm{C} 24-\mathrm{C} 25-\mathrm{C} 20$ & $118.8(6)$ \\
\hline $\mathrm{C} 2-\mathrm{C} 1-\mathrm{C} 3$ & $62.7(4)$ & $\mathrm{F} 3 \mathrm{~B}-\mathrm{C} 26-\mathrm{F} 1 \mathrm{~B}$ & $109.8(8)$ \\
\hline $\mathrm{C} 2-\mathrm{C} 1-\mathrm{N} 3$ & $146.6(5)$ & $\mathrm{F} 1 \mathrm{~A}-\mathrm{C} 26-\mathrm{F} 3 \mathrm{~A}$ & $108.7(8)$ \\
\hline $\mathrm{C} 3-\mathrm{C} 1-\mathrm{N} 3$ & $150.3(5)$ & $\mathrm{F} 3 \mathrm{~B}-\mathrm{C} 26-\mathrm{F} 2 \mathrm{~B}$ & $104.8(6)$ \\
\hline $\mathrm{N} 1-\mathrm{C} 2-\mathrm{C} 1$ & $148.2(6)$ & $\mathrm{F} 1 \mathrm{~B}-\mathrm{C} 26-\mathrm{F} 2 \mathrm{~B}$ & $103.5(7)$ \\
\hline $\mathrm{N} 1-\mathrm{C} 2-\mathrm{C} 3$ & $152.9(6)$ & $\mathrm{F} 1 \mathrm{~A}-\mathrm{C} 26-\mathrm{F} 2 \mathrm{~A}$ & $104.2(8)$ \\
\hline $\mathrm{C} 1-\mathrm{C} 2-\mathrm{C} 3$ & $58.9(4)$ & $\mathrm{F} 3 \mathrm{~A}-\mathrm{C} 26-\mathrm{F} 2 \mathrm{~A}$ & $104.6(6)$ \\
\hline $\mathrm{N} 2-\mathrm{C} 3-\mathrm{C} 1$ & $149.1(6)$ & $\mathrm{F} 3 \mathrm{~B}-\mathrm{C} 26-\mathrm{C} 24$ & $114.0(6)$ \\
\hline $\mathrm{N} 2-\mathrm{C} 3-\mathrm{C} 2$ & $152.5(6)$ & $\mathrm{F} 1 \mathrm{~B}-\mathrm{C} 26-\mathrm{C} 24$ & $115.2(8)$ \\
\hline $\mathrm{C} 1-\mathrm{C} 3-\mathrm{C} 2$ & $58.4(4)$ & $\mathrm{F} 1 \mathrm{~A}-\mathrm{C} 26-\mathrm{C} 24$ & $112.9(9)$ \\
\hline $\mathrm{N} 1-\mathrm{C} 4-\mathrm{C} 6$ & $111.4(5)$ & $\mathrm{F} 3 \mathrm{~A}-\mathrm{C} 26-\mathrm{C} 24$ & $116.1(7)$ \\
\hline $\mathrm{N} 1-\mathrm{C} 4-\mathrm{C} 5$ & $111.2(5)$ & $\mathrm{F} 2 \mathrm{~B}-\mathrm{C} 26-\mathrm{C} 24$ & $108.5(7)$ \\
\hline $\mathrm{C} 6-\mathrm{C} 4-\mathrm{C} 5$ & $113.5(6)$ & $\mathrm{F} 2 \mathrm{~A}-\mathrm{C} 26-\mathrm{C} 24$ & $109.3(6)$ \\
\hline
\end{tabular}




\begin{tabular}{cccc}
\hline $\mathrm{N} 1-\mathrm{C} 7-\mathrm{C} 9$ & $111.9(6)$ & $\mathrm{F} 4 \mathrm{~B}-\mathrm{C} 27-\mathrm{F} 5 \mathrm{~B}$ & $109.7(8)$ \\
$\mathrm{N} 1-\mathrm{C} 7-\mathrm{C} 8$ & $110.1(5)$ & $\mathrm{F} 5 \mathrm{~A}-\mathrm{C} 27-\mathrm{F} 6 \mathrm{~A}$ & $106.7(7)$ \\
$\mathrm{C} 9-\mathrm{C} 7-\mathrm{C} 8$ & $112.8(6)$ & $\mathrm{F} 6 \mathrm{C}-\mathrm{C} 27-\mathrm{F} 4 \mathrm{C}$ & $106.3(10)$ \\
$\mathrm{C} 12-\mathrm{C} 10-\mathrm{N} 2$ & $111.3(6)$ & $\mathrm{F} 5 \mathrm{~A}-\mathrm{C} 27-\mathrm{F} 4 \mathrm{~A}$ & $106.4(7)$ \\
$\mathrm{C} 12-\mathrm{C} 10-\mathrm{C} 11$ & $117.0(7)$ & $\mathrm{F} 6 \mathrm{~A}-\mathrm{C} 27-\mathrm{F} 4 \mathrm{~A}$ & $105.4(7)$ \\
$\mathrm{N} 2-\mathrm{C} 10-\mathrm{C} 11$ & $109.6(6)$ & $\mathrm{F} 4 \mathrm{~B}-\mathrm{C} 27-\mathrm{F} 6 \mathrm{~B}$ & $104.7(8)$ \\
$\mathrm{N} 2-\mathrm{C} 13-\mathrm{C} 14$ & $111.4(5)$ & $\mathrm{F} 5 \mathrm{~B}-\mathrm{C} 27-\mathrm{F} 6 \mathrm{~B}$ & $104.5(8)$ \\
$\mathrm{N} 2-\mathrm{C} 13-\mathrm{C} 15$ & $111.4(5)$ & $\mathrm{F} 6 \mathrm{C}-\mathrm{C} 27-\mathrm{F} 5 \mathrm{C}$ & $102.7(10)$ \\
$\mathrm{C} 14-\mathrm{C} 13-\mathrm{C} 15$ & $110.6(5)$ & $\mathrm{F} 4 \mathrm{C}-\mathrm{C} 27-\mathrm{F} 5 \mathrm{C}$ & $102.1(10)$ \\
$\mathrm{N} 3-\mathrm{C} 16-\mathrm{C} 19$ & $133.8(5)$ & $\mathrm{F} 4 \mathrm{~B}-\mathrm{C} 27-\mathrm{C} 22$ & $112.8(7)$ \\
$\mathrm{N} 3-\mathrm{C} 16-\mathrm{C} 17$ & $132.9(5)$ & $\mathrm{F} 5 \mathrm{~B}-\mathrm{C} 27-\mathrm{C} 22$ & $114.6(7)$ \\
$\mathrm{C} 19-\mathrm{C} 16-\mathrm{C} 17$ & $93.2(5)$ & $\mathrm{F} 5 \mathrm{~A}-\mathrm{C} 27-\mathrm{C} 22$ & $114.9(6)$ \\
$\mathrm{O} 1-\mathrm{C} 17-\mathrm{C} 16$ & $135.6(6)$ & $\mathrm{F} 6 \mathrm{C}-\mathrm{C} 27-\mathrm{C} 22$ & $115.3(9)$ \\
$\mathrm{O} 1-\mathrm{C} 17-\mathrm{C} 18$ & $137.4(6)$ & $\mathrm{F} 6 \mathrm{~A}-\mathrm{C} 27-\mathrm{C} 22$ & $111.9(6)$ \\
$\mathrm{C} 16-\mathrm{C} 17-\mathrm{C} 18$ & $87.0(5)$ & $\mathrm{F} 4 \mathrm{C}-\mathrm{C} 27-\mathrm{C} 22$ & $113.7(9)$ \\
$\mathrm{O} 2-\mathrm{C} 18-\mathrm{C} 19$ & $137.7(7)$ & $\mathrm{F} 4 \mathrm{~A}-\mathrm{C} 27-\mathrm{C} 22$ & $111.0(6)$ \\
$\mathrm{O} 2-\mathrm{C} 18-\mathrm{C} 17$ & $134.2(6)$ & $\mathrm{F} 6 \mathrm{~B}-\mathrm{C} 27-\mathrm{C} 22$ & $109.8(7)$ \\
$\mathrm{C} 19-\mathrm{C} 18-\mathrm{C} 17$ & $88.1(5)$ & $\mathrm{F} 5 \mathrm{C}-\mathrm{C} 27-\mathrm{C} 22$ & $115.2(9)$ \\
$\mathrm{N} 4-\mathrm{C} 19-\mathrm{C} 16$ & $128.9(6)$ & & \\
\hline
\end{tabular}

Table S8 Summary of H-bonding interactions present in $\mathbf{4 e} \cdot \mathbf{B F} \mathbf{F}_{\mathbf{4}}^{-}$.

\begin{tabular}{|c|c|c|c|}
\hline Donor-H...Acceptor & H...A (A) & D...A (Å) & D-H.....A ( $\left(^{\circ}\right)$ \\
\hline N3-H31...F10 & 1.95 & $2.760(6)$ & 153 \\
\hline N4-H41 ...F10 & 2.02 & $2.867(7)$ & 160 \\
\hline C4-H4....F6C & 2.43 & $3.373(19)$ & 157 \\
\hline C13-H13...F $1 B$ & 2.51 & $3.287(14)$ & 134 \\
\hline C14-H14A....O1 & 2.47 & $3.086(8)$ & 120 \\
\hline C15-H15B...F7 & 2.49 & $3.238(7)$ & 134 \\
\hline $\mathrm{C} 21-\mathrm{H} 21 \ldots \mathrm{O} 2$ & 2.17 & $3.061(8)$ & 156 \\
\hline $\mathrm{C} 23-\mathrm{H} 23 \ldots \mathrm{F} 5 \mathrm{~A}$ & 2.41 & $2.733(11)$ & 100 \\
\hline $\mathrm{C} 25-\mathrm{H} 25 \ldots \mathrm{F} 7$ & 2.28 & $3.216(7)$ & 166 \\
\hline
\end{tabular}

\section{Computational details}

DFT calculations were performed using the software package Gaussian 09, Revision E.0131 employing the range-separated hybrid $\omega$ B97X-D functional ${ }^{32}$ with a $6-31 \mathrm{G}(\mathrm{d})$ basis set. This functional was selected as it accounts for dispersion and has been shown to provide accurate thermochemical and kinetic energies. The optimized geometries were verified as transition state structures (one imaginary frequency) or minima (zero imaginary frequencies) by frequency calculations. Intrinsic reaction coordinate calculations ${ }^{33,34}$ were performed to confirm that all transition state structures were linked to relevant minima. Notably, numerous transition state geometries and addition modes were considered, from which the lowest energy models from each pathway were included. The energies of the $\omega \mathrm{B} 97 \mathrm{X}-\mathrm{D} / 6-31 \mathrm{G}(\mathrm{d})$ optimized structures were further refined by single-point calculations performed at the $\omega \mathrm{B} 97 \mathrm{X}-\mathrm{D} / 6-311++\mathrm{G}(\mathrm{d}, \mathrm{p})$ level of theory 
using the integral equation formalism polarizable continuum model (IEFPCM) with the default parameters of dichloromethane $(\varepsilon=8.93)$ to account for solvent. ${ }^{35}$ The final reported Gibbs free energies are the summed thermal corrections to the Gibbs free energies computed at the lower level of theory $(\omega \mathrm{B} 97 \mathrm{X}-\mathrm{D} / 6-31 \mathrm{G}(\mathrm{d}))$ and electronic energies from single-point calculations $(\omega \mathrm{B} 97 \mathrm{X}-\mathrm{D} / 6-311++\mathrm{G}(\mathrm{d}, \mathrm{p}))$. The keyword (integral=grid=ultrafine) was used for all calculations pertaining to the reaction coordinate (transition state and minima). A Wiberg bond index (WBI) single-point $(\omega \mathrm{B} 97 \mathrm{X}-\mathrm{D} / 6-311++\mathrm{G}(\mathrm{d}, \mathrm{p}))$ was performed using the optimized geometry of structure 4e. The natural bond orbital (NBO) charges of structure $4 \mathbf{e}$ were obtained using the B3LYP/6$31+\mathrm{G}(\mathrm{d}, \mathrm{p})$ level of theory. A further NBO analysis on TS1 using second-order perturbation theory was employed to probe the degree of charge transfer involving oxygen lone pair donation into the antibonding $\sigma^{*}$-orbitals of the $\mathrm{N}-\mathrm{H}$ bond of catalyst $4 \mathbf{e}$. The $\mathrm{p} K_{\mathrm{a}}$ was calculated in accordance to the method of Xue and $\mathrm{Ji}^{36}$ at the $\mathrm{SMD}_{(\mathrm{DMSO})} \mathrm{M} 06-2 \mathrm{X} / 6-311++\mathrm{G}(2 \mathrm{df}, 2 \mathrm{p}) / / \mathrm{B} 3 \mathrm{LYP} / 6-31+\mathrm{G}(\mathrm{d})$ level of theory. Notably, this method has been successfully applied to predict the $\mathrm{p} K_{\mathrm{a}}$ values of numerous $\mathrm{O}-\mathrm{H}$ and $\mathrm{N}-\mathrm{H}$ acids in DMSO. ${ }^{37}$ The 3D images of all optimized geometries were generated with CYLview, ${ }^{38}$ and GaussView $6.1^{39}$ was used to construct all structures prior to optimization and visualize the output from the Gaussian 09 calculations. The NBO image was generated using Chemcraft. ${ }^{40}$ The reported NCI plots (isovalue $=0.3$, $\min =-0.05$ and $\max =0.05$ ), highest occupied molecular orbital (HOMO) and HOMO-1 images (isovalue $=-0.5$ ), and electrostatic potential $(\mathrm{ESP})$ surface (isovalue $=0.0001$ ) were calculated using the B3LYP-D3 functional with a LACVP+*41-43 basis set using the program Jaguar of the Schrödinger software package. ${ }^{44,45}$ Monte Carlo conformational searches (MCCS) with an OPLS4 force field were performed on the full catalyst systems using the Schrödinger MacroModel program to locate conformers submitted for DFT optimization. ${ }^{46}$

\section{Cartesian coordinates and energies of calculated structures}

Table S9. Single-point energies of all structures calculated at the $\mathrm{SMD}_{(\mathrm{DMSO})} \mathrm{M} 06-2 \mathrm{X} / 6-311++\mathrm{G}(2 \mathrm{df}, 2 \mathrm{p})$ level of theory and thermal correction to Gibbs Free Energy and Gibbs Free Energy calculated at the B3LYP/6-31+G(d) level of theory. All energies are reported in Hartrees.

\begin{tabular}{|c|c|c|c|c|}
\hline Structure & $\begin{array}{c}\text { Single-Point } \\
\text { Energies (E) } \\
\text { SMD }_{(\text {DMSO) }} \text { M06- } \\
\text { 2X/6- } \\
\text { 311++G(2df,2p) }\end{array}$ & $\begin{array}{c}\text { Single-Point } \\
\text { Energies (E) } \\
\text { M06-2X/6- } \\
\text { 311++G(2df,2p) }\end{array}$ & $\begin{array}{c}\text { Thermal } \\
\text { Corrections to } \\
\text { Gibbs Free } \\
\text { Energies (G) } \\
\text { B3LYP/6- } \\
\text { 31+G(d) }\end{array}$ & $\begin{array}{c}\text { Gibbs Free } \\
\text { Energies (G) } \\
\text { B3LYP/6- } \\
\text { 31+G(d) }\end{array}$ \\
\hline
\end{tabular}




\begin{tabular}{|c|c|c|c|c|}
\hline $\mathbf{4 e}$ & -2016.98993189 & -2016.88524360 & 0.492522 & -2016.550047 \\
\hline $\mathbf{4 e}_{\mathbf{c y}-\mathbf{H}}$ & -2016.53535221 & -2016.49826211 & 0.480739 & -2016.178015 \\
\hline $\mathbf{4 e}_{\text {arvl-H }}$ & -2016.52083588 & -2016.46934443 & 0.478187 & -2016.151713 \\
\hline
\end{tabular}

Structure 4e

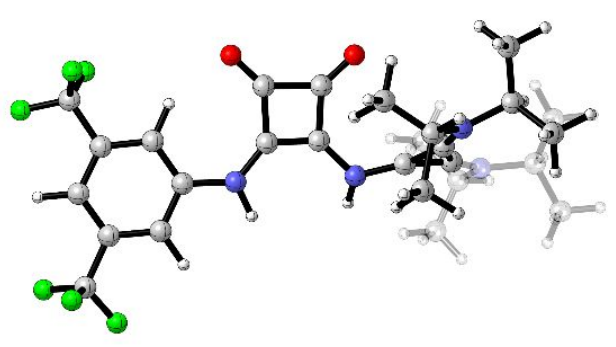

Number of Imaginary Frequencies $=0$

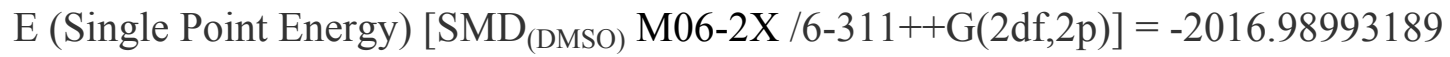

Zero-point correction=

Thermal correction to Energy=

Thermal correction to Enthalpy=

Thermal correction to Gibbs Free Energy=

Sum of electronic and zero-point Energies=

Sum of electronic and thermal Energies=

Sum of electronic and thermal Enthalpies=

Sum of electronic and thermal Free Energies=
0.574965 (Hartree/Particle)

0.616824

0.617769

0.492522

$-2016.467604$

$-2016.425745$

$-2016.424800$

$-2016.550047$

$\begin{array}{lrrr}11 & & & \\ \mathrm{C} & 0.91552300 & -0.38234500 & -0.35415800 \\ \mathrm{C} & -0.47315600 & -0.25792900 & -0.36787400 \\ \mathrm{C} & -0.70735100 & -1.51646900 & -1.09215700 \\ \mathrm{C} & 0.82815900 & -1.70854300 & -1.10543700 \\ \mathrm{O} & 1.60769900 & -2.51031600 & -1.54706900 \\ \mathrm{O} & -1.68383300 & -2.11400400 & -1.48953700 \\ \mathrm{~N} & -1.34826700 & 0.72720900 & 0.06726100 \\ \mathrm{H} & -1.08698500 & 1.69443100 & -0.10585500 \\ \mathrm{C} & -2.70227400 & 0.48088500 & 0.13256300 \\ \mathrm{C} & -3.98155300 & 0.82876600 & -0.24746800 \\ \mathrm{C} & -3.74780000 & -0.27953700 & 0.62113700 \\ \mathrm{~N} & -4.22537800 & -1.27863000 & 1.33585600 \\ \mathrm{~N} & -4.79598700 & 1.62320400 & -0.91587200 \\ \mathrm{C} & -4.22796800 & 2.61083100 & -1.88775900 \\ \mathrm{C} & -3.45015500 & 3.72199600 & -1.17207100 \\ \mathrm{C} & -3.40997800 & 1.91370700 & -2.98061000 \\ \mathrm{H} & -2.55011600 & 3.33481800 & -0.67798600 \\ \mathrm{H} & -3.12389900 & 4.47445200 & -1.89754500\end{array}$




\begin{tabular}{|c|c|c|c|}
\hline $\mathrm{H}$ & -4.06342700 & 4.22013100 & -0.41524400 \\
\hline $\mathrm{H}$ & -2.50645700 & 1.44111700 & -2.57884300 \\
\hline $\mathrm{H}$ & -3.99843900 & 1.14211700 & -3.48647700 \\
\hline $\mathrm{H}$ & -3.09447400 & 2.64774200 & -3.72951200 \\
\hline $\mathrm{C}$ & -6.27735900 & 1.54088000 & -0.72519700 \\
\hline $\mathrm{C}$ & -6.97247100 & 1.01135000 & -1.98499000 \\
\hline $\mathrm{H}$ & -6.87706300 & 1.70574700 & -2.82702000 \\
\hline $\mathrm{H}$ & -6.56268700 & 0.04284200 & -2.29098200 \\
\hline $\mathrm{H}$ & -8.04240900 & 0.88381300 & -1.78876300 \\
\hline $\mathrm{C}$ & -5.62741200 & -1.75494500 & 1.11357800 \\
\hline $\mathrm{C}$ & -5.64636300 & -3.23399300 & 0.70658500 \\
\hline $\mathrm{C}$ & -6.52714200 & -1.45412200 & 2.31769700 \\
\hline $\mathrm{H}$ & -5.32582000 & -3.89184700 & 1.52132200 \\
\hline $\mathrm{H}$ & -6.66971500 & -3.52055100 & 0.44155000 \\
\hline $\mathrm{H}$ & -5.00428500 & -3.41428800 & -0.16119100 \\
\hline $\mathrm{H}$ & -7.55836900 & -1.74114300 & 2.08564300 \\
\hline $\mathrm{H}$ & -6.22579900 & -2.01911900 & 3.20637200 \\
\hline $\mathrm{H}$ & -6.51784000 & -0.38806800 & 2.57085000 \\
\hline $\mathrm{C}$ & -3.39602800 & -1.92772700 & 2.40461000 \\
\hline $\mathrm{C}$ & -2.22712700 & -2.74343800 & 1.84617100 \\
\hline $\mathrm{H}$ & -1.43264200 & -2.09431200 & 1.46608200 \\
\hline $\mathrm{H}$ & -1.79618400 & -3.34960900 & 2.65038000 \\
\hline $\mathrm{H}$ & -2.54164600 & -3.41065500 & 1.03987400 \\
\hline $\mathrm{N}$ & 1.87137800 & 0.40647700 & 0.16911100 \\
\hline $\mathrm{H}$ & 1.54371600 & 1.18313600 & 0.73524700 \\
\hline $\mathrm{H}$ & -5.98525300 & -1.17893500 & 0.25611500 \\
\hline $\mathrm{H}$ & -5.10178400 & 3.06490300 & -2.36009500 \\
\hline $\mathrm{H}$ & -4.08795700 & -2.61896100 & 2.89016100 \\
\hline $\mathrm{C}$ & -2.94802600 & -0.89558300 & 3.44593000 \\
\hline $\mathrm{H}$ & -3.80143100 & -0.35633400 & 3.87005600 \\
\hline $\mathrm{H}$ & -2.42474100 & -1.40145100 & 4.26420400 \\
\hline $\mathrm{H}$ & -2.25420300 & -0.16428700 & 3.01402400 \\
\hline $\mathrm{H}$ & -6.41497700 & 0.81541300 & 0.08056900 \\
\hline $\mathrm{C}$ & -6.85598600 & 2.87757100 & -0.24403200 \\
\hline $\mathrm{H}$ & -6.35783300 & 3.22363700 & 0.66764300 \\
\hline $\mathrm{H}$ & -6.77767700 & 3.66214700 & -1.00420200 \\
\hline $\mathrm{H}$ & -7.92032800 & 2.74929300 & -0.02062600 \\
\hline $\mathrm{C}$ & 3.28786600 & 0.30802500 & 0.10816400 \\
\hline $\mathrm{C}$ & 3.95494100 & -0.66512100 & -0.63873000 \\
\hline $\mathrm{C}$ & 4.01465300 & 1.26166500 & 0.83364900 \\
\hline $\mathrm{C}$ & 5.35316500 & -0.66996100 & -0.64732700 \\
\hline $\mathrm{H}$ & 3.40819300 & -1.41557900 & -1.19967600 \\
\hline $\mathrm{C}$ & 5.40617900 & 1.23959200 & 0.80852500 \\
\hline $\mathrm{H}$ & 3.50081400 & 2.02422500 & 1.41262900 \\
\hline $\mathrm{C}$ & 6.09068000 & 0.27186800 & 0.06786500 \\
\hline $\mathrm{H}$ & 7.17384000 & 0.25902200 & 0.04409700 \\
\hline
\end{tabular}




$\begin{array}{lrrc}\text { C } & 6.18320000 & 2.24141000 & 1.63160500 \\ \text { C } & 6.05198300 & -1.74463500 & -1.45297500 \\ \text { F } & 6.50773200 & 1.73303400 & 2.84629300 \\ \text { F } & 7.33492900 & 2.60233200 & 1.02911600 \\ \text { F } & 5.46695300 & 3.37110100 & 1.85626400 \\ F & 7.38755600 & -1.55957600 & -1.49984500 \\ \text { F } & 5.82950800 & -2.97237300 & -0.92507000 \\ \text { F } & 5.59838200 & -1.77477500 & -2.72878000\end{array}$

Structure $\mathbf{4} \mathbf{e}_{\mathrm{cy}-\mathrm{H}}$

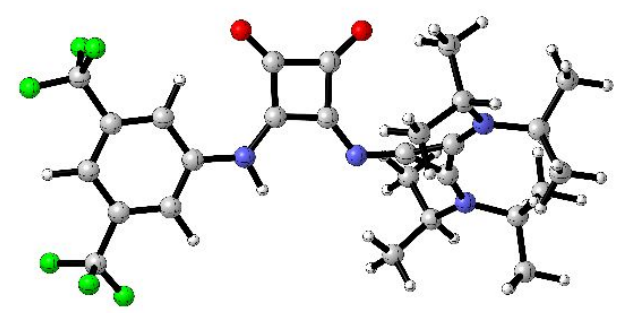

Zero-point correction $=$

Thermal correction to Energy=

Thermal correction to Enthalpy=

Thermal correction to Gibbs Free Energy=

Sum of electronic and zero-point Energies=

Sum of electronic and thermal Energies=

Sum of electronic and thermal Enthalpies=

Sum of electronic and thermal Free Energies=
0.562318 (Hartree/Particle)

0.603574

0.604518

0.480739

$-2016.096437$

$-2016.055181$

$-2016.054237$

$-2016.178015$

Number of Imaginary Frequencies $=0$

E (Single Point Energy) [SMD ${ }_{(\mathrm{DMSO})}$ M06-2X /6-311++G(2df,2p)] = -2016.53535221

01

C $\quad 0.92518800 \quad-0.81136800 \quad-0.52066000$

$\begin{array}{llll}\mathrm{C} & -0.48256600 & -0.77375500 & -0.58522100\end{array}$

$\begin{array}{llll}\mathrm{C} & -0.52078400 & -2.12939300 & -1.21071400\end{array}$

C $\quad 1.00948400 \quad-2.17481800-1.08584200$

O $\quad 1.89022200 \quad-2.97639800-1.35703700$

$\begin{array}{llll}\mathrm{O} & -1.39371000 & -2.85744400 & -1.64945300\end{array}$

$\mathrm{N} \quad-1.30682300 \quad 0.24031600-0.32344300$

$\begin{array}{llll}\mathrm{C} & -2.62292900 & 0.10843300 & -0.18923200\end{array}$

$\begin{array}{llll}\text { C } & -3.82741700 & 0.79576300 & -0.30270200\end{array}$

$\begin{array}{llll}\mathrm{C} & -3.80728800 & -0.45764600 & 0.31524000\end{array}$

$\mathrm{N} \quad-4.47324900 \quad-1.42112100 \quad 0.96874700$

$\mathrm{N} \quad \begin{array}{llll}\mathrm{N} & -4.44933500 & 1.90191800 & -0.73587500\end{array}$

$\begin{array}{llll}\text { C } & -3.67372400 & 2.87909700 & -1.55012500\end{array}$

$\begin{array}{lllll}\text { C } & -2.56024100 & 3.54279600 & -0.72692900\end{array}$ 


$\begin{array}{lrrr}\mathrm{C} & -3.13603200 & 2.24061600 & -2.83821000 \\ \mathrm{H} & -1.80497900 & 2.80689300 & -0.43083000 \\ \mathrm{H} & -2.06465800 & 4.31598300 & -1.32605000 \\ \mathrm{H} & -2.96398000 & 4.01652100 & 0.17469900 \\ \mathrm{H} & -2.38024600 & 1.47962800 & -2.61853000 \\ \mathrm{H} & -3.94224800 & 1.77618100 & -3.41642600 \\ \mathrm{H} & -2.66466100 & 3.00771200 & -3.46348100 \\ \mathrm{C} & -5.87726200 & 2.15584100 & -0.44061800 \\ \mathrm{C} & -6.74556500 & 2.08515100 & -1.70608800 \\ \mathrm{H} & -6.49329800 & 2.88255000 & -2.41457000 \\ \mathrm{H} & -6.61941400 & 1.12407000 & -2.21627400 \\ \mathrm{H} & -7.80401400 & 2.20008500 & -1.44456200 \\ \mathrm{C} & -5.94998400 & -1.32503900 & 1.08259900 \\ \mathrm{C} & -6.65342200 & -2.65358600 & 0.77339300 \\ \mathrm{C} & -6.37946100 & -0.74774600 & 2.44116300 \\ \mathrm{H} & -6.43703000 & -3.42226200 & 1.52289800 \\ \mathrm{H} & -7.73787600 & -2.49452800 & 0.77155200 \\ \mathrm{H} & -6.36136700 & -3.03527100 & -0.20970700 \\ \mathrm{H} & -7.46668900 & -0.60800700 & 2.46915700 \\ \mathrm{H} & -6.11221900 & -1.41926200 & 3.26519800 \\ \mathrm{H} & -5.90372000 & 0.22174400 & 2.62725700 \\ \mathrm{C} & -3.75307000 & -2.47699700 & 1.74061200 \\ \mathrm{C} & -3.39341900 & -3.68936400 & 0.87220900 \\ \mathrm{H} & -2.74078400 & -3.41021000 & 0.04073400 \\ \mathrm{C} & -2.87393200 & -4.43919400 & 1.48135000 \\ \mathrm{H} & -4.28875100 & -4.15941000 & 0.45420100 \\ \mathrm{H} & 1.74397600 & 0.17725700 & -0.06743200 \\ \mathrm{~N} & 1.24949800 & 1.01220500 & 0.23339900 \\ \mathrm{H} & -6.24941000 & -0.62452500 & 0.29852500 \\ \mathrm{H} & -4.39884800 & 3.64831600 & -1.83121400 \\ \mathrm{H} & -4.47292000 & -2.79710200 & 2.50188200 \\ \mathrm{H} & -2.53664800 & -1.91089800 & 2.48435700 \\ \mathrm{C} & -2.80960200 & -1.03961400 & 3.09079000 \\ \mathrm{H} & -2.13309600 & -2.67906300 & 3.15303300 \\ \mathrm{H} & -1.73569100 & -1.61810000 & 1.79937000 \\ \mathrm{H} & -6.18037300 & 1.34406400 & 0.22446600 \\ \mathrm{H} & -6.08065100 & 3.46924100 & 0.33083700 \\ \mathrm{H} & -5.47464700 & 3.48676300 & 1.24282600 \\ \mathrm{H} & -5.82013500 & 4.34629300 & -0.27206200 \\ \mathrm{H} & 3.13796800 & 3.57019300 & 0.61678000 \\ \mathrm{H} & 0.22269200 & 0.02574800 \\ \mathrm{H} & -0.84859800 & -0.35671200 \\ \mathrm{H} & -3294300 & 1.39911300 & 0.52643400 \\ \mathrm{H} & -0.72230100 & -0.23163700 \\ \mathrm{H} & -1.76462800 & -0.74782100 \\ \mathrm{H} & -1.50005700 & 0.63887200\end{array}$




$\begin{array}{lccc}\mathrm{H} & 3.09428500 & 2.23439100 & 0.82139700 \\ \mathrm{C} & 5.93743800 & 0.44041300 & 0.26219100 \\ \mathrm{H} & 7.01376900 & 0.52229800 & 0.34841000 \\ \mathrm{C} & 5.71708400 & 2.74567100 & 1.22711200 \\ \mathrm{C} & 6.20545300 & -1.88421700 & -0.66994500 \\ \mathrm{~F} & 6.90991300 & 3.04842400 & 0.66082900 \\ \mathrm{~F} & 4.91755400 & 3.83270700 & 1.07971000 \\ \mathrm{~F} & 5.94538400 & 2.61369400 & 2.56278700 \\ \mathrm{~F} & 7.51771700 & -1.68969500 & -0.38712600 \\ \mathrm{~F} & 5.83730900 & -3.04018300 & -0.06686100 \\ \mathrm{~F} & 6.11884300 & -2.09578400 & -2.00770000\end{array}$

Structure $\mathbf{4} \mathbf{e}_{\text {aryl-H }}$

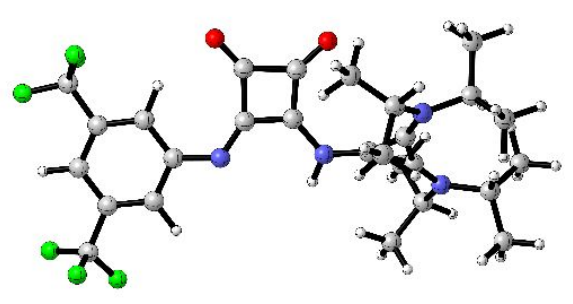

Zero-point correction $=$

0.561308 (Hartree/Particle)

Thermal correction to Energy=

0.602892

Thermal correction to Enthalpy=

0.603836

Thermal correction to Gibbs Free Energy=

0.478187

Sum of electronic and zero-point Energies=

$-2016.068592$

Sum of electronic and thermal Energies $=$

$-2016.027008$

Sum of electronic and thermal Enthalpies=

$-2016.026064$

Sum of electronic and thermal Free Energies=

$-2016.151713$

Number of Imaginary Frequencies $=0$

E (Single Point Energy) $\left[\mathrm{SMD}_{(\mathrm{DMSO})} \mathrm{M} 06-2 \mathrm{X} / 6-311++\mathrm{G}(2 \mathrm{df}, 2 \mathrm{p})\right]=-2016.52083588$

01

C $\quad 1.00512000 \quad-0.68779200 \quad-0.33365900$

$\begin{array}{llll}\text { C } & -0.41627800 & -0.68636900 & -0.38200800\end{array}$

C $\quad-0.58236700 \quad-1.94079100 \quad-1.05907300$

C $\quad 0.95676200 \quad-2.08975600 \quad-0.99315800$

O $\quad 1.75982200-2.92146800-1.35254000$

$\mathrm{O} \quad-1.52171100-2.59447700-1.51535600$

$\begin{array}{llll}\mathrm{N} & -1.26284600 & 0.37813800 & -0.08045200\end{array}$

$\begin{array}{llll}\mathrm{C} & -2.59742000 & 0.29593300 & 0.02955200\end{array}$

C $\quad-3.83621200 \quad 0.87735900 \quad-0.19873700$

C $\quad-3.75001000 \quad-0.38373200 \quad 0.41399100$

$\mathrm{N} \quad-4.37362100 \quad-1.42775000 \quad 0.95522100$ 


$\begin{array}{lrrr}\mathrm{N} & -4.54407100 & 1.89487300 & -0.68535300 \\ \mathrm{C} & -3.86782000 & 2.93666500 & -1.51068200 \\ \mathrm{C} & -2.90700600 & 3.79219700 & -0.67315400 \\ \mathrm{C} & -3.19243200 & 2.32462200 & -2.74456300 \\ \mathrm{H} & -2.06438600 & 3.20358700 & -0.29326400 \\ \mathrm{H} & -2.48978000 & 4.59721400 & -1.28790400 \\ \mathrm{H} & -3.41743200 & 4.24376400 & 0.18332700 \\ \mathrm{H} & -2.36489200 & 1.66134000 & -2.47072600 \\ \mathrm{H} & -3.90727600 & 1.74731400 & -3.34002400 \\ \mathrm{H} & -2.78347100 & 3.12031500 & -3.37686900 \\ \mathrm{C} & -6.00055500 & 1.99404600 & -0.40391100 \\ \mathrm{C} & -6.83703600 & 1.83157800 & -1.68059000 \\ \mathrm{H} & -6.67410200 & 2.65728300 & -2.38251600 \\ \mathrm{H} & -6.59740500 & 0.89464300 & -2.19476100 \\ \mathrm{H} & -7.90341600 & 1.82211400 & -1.42845700 \\ \mathrm{C} & -5.77501200 & -1.72215000 & 0.55071100 \\ \mathrm{C} & -5.87406400 & -3.10676300 & -0.10645100 \\ \mathrm{C} & -6.77046800 & -1.54522200 & 1.70566500 \\ \mathrm{H} & -5.68065500 & -3.91307000 & 0.60968200 \\ \mathrm{H} & -6.88512300 & -3.25487000 & -0.50321200 \\ \mathrm{H} & -5.15843000 & -3.19980500 & -0.92911800 \\ \mathrm{H} & -7.79299200 & -1.68903300 & 1.33829900 \\ \mathrm{H} & -6.60465100 & -2.27731900 & 2.50361800 \\ \mathrm{H} & -6.70168500 & -0.54345800 & 2.14488300 \\ \mathrm{C} & -3.72333200 & -2.26246500 & 2.01297800 \\ \mathrm{C} & -2.49820500 & -3.03693200 & 1.52475700 \\ \mathrm{C} & -1.64091000 & -2.37552000 & 1.36997800 \\ \mathrm{H} & -2.21361100 & -3.76980800 & 2.28874000 \\ \mathrm{H} & -2.68883100 & -3.55958300 & 0.58505800 \\ \mathrm{H} & 1.78167800 & 0.26656100 & 0.09236700 \\ \mathrm{~N} & -6.00843700 & -0.98119300 & -0.22039300 \\ \mathrm{H} & -4.67663200 & 3.58555600 & -1.85757400 \\ \mathrm{H} & -4.49378600 & -2.98846900 & 2.28529300 \\ \mathrm{H} & -3.41036200 & -1.41059600 & 3.25110200 \\ \mathrm{H} & -4.30515100 & -0.90459500 & 3.63037100 \\ \mathrm{H} & -3.01107500 & -2.04631800 & 4.04918500 \\ \mathrm{H} & -2.65099100 & -0.65215000 & 3.02583600 \\ \mathrm{H} & -6.21730500 & 1.15023500 & 0.25717800 \\ \mathrm{H} & -6.34586800 & 3.27905600 & 0.36217100 \\ \mathrm{H} & -6.16731800 & 4.17710000 & -0.23975300 \\ \mathrm{H} & 3.40807800 & 3.27225800 & 0.63113200 \\ \mathrm{H} & 0.21857700 & 0.07811800 \\ \mathrm{H} & -0.86312100 & -0.35216900 \\ \mathrm{H} & & & \\ \mathrm{H} & -3.37354800 & 0.55187800 \\ \mathrm{H} & -0.77029700 & -0.30214800\end{array}$




$\begin{array}{lccc}\mathrm{H} & 3.50761500 & -1.77047000 & -0.72577900 \\ \mathrm{C} & 5.22016800 & 1.45073700 & 0.58944500 \\ \mathrm{H} & 3.21893500 & 2.20569400 & 0.88482700 \\ \mathrm{C} & 6.01045500 & 0.37823800 & 0.16393600 \\ \mathrm{H} & 7.09128800 & 0.43677600 & 0.19327300 \\ \mathrm{C} & 5.87447600 & 2.68269600 & 1.15268500 \\ \mathrm{C} & 6.18049700 & -1.93507800 & -0.80571800 \\ \mathrm{~F} & 7.12971600 & 2.87264600 & 0.67361700 \\ \mathrm{~F} & 5.17324300 & 3.81487900 & 0.87989200 \\ \mathrm{~F} & 5.98835200 & 2.62770700 & 2.51135600 \\ \mathrm{~F} & 7.49433300 & -1.83494400 & -0.47466700 \\ \mathrm{~F} & 5.74141600 & -3.12031700 & -0.31372700 \\ \mathrm{~F} & 6.13229500 & -2.04073000 & -2.16224500 \\ \mathrm{H} & -0.82863600 & 1.29839400 & -0.11791200\end{array}$

Table S10. Single-point energies of all structures calculated at the $\operatorname{IEFPCM}_{(\mathrm{DCM})} \omega \mathrm{B} 97 \mathrm{X}-\mathrm{D} / 6-$ $311++\mathrm{G}(\mathrm{d}, \mathrm{p})$ level of theory and thermal correction to Gibbs Free Energy and Gibbs Free Energy calculated at the $\omega \mathrm{B} 97 \mathrm{X}-\mathrm{D} / 6-31 \mathrm{G}(\mathrm{d})$ level of theory. All energies are reported in Hartrees.

\begin{tabular}{|c|c|c|c|c|}
\hline Structure & $\begin{array}{c}\text { Single-Point } \\
\text { Energies (E) } \\
\text { IEFPCM }_{(\mathrm{DCM})} \omega \\
\text { B97X-D/6- }^{\omega} \\
\text { 311++G(d,p) }\end{array}$ & $\begin{array}{c}\text { Thermal } \\
\text { Corrections to } \\
\text { Gibbs Free } \\
\text { Energies (G) } \\
\omega \mathrm{B} 97 \mathrm{X}-\mathrm{D} / 6- \\
\mathbf{3 1 G ( d )} \\
\end{array}$ & $\begin{array}{c}\text { Gibbs Free } \\
\text { Energies (G) } \\
\omega B 97 X-D / 6- \\
31 G(d)\end{array}$ & $\begin{array}{c}\text { Gibbs Free Energies } \\
(G) \\
\text { IEFPCM }_{(\mathrm{DCM})} \omega \mathrm{B} 97 \mathrm{X}- \\
\text { D/6- } \\
311++\mathrm{G}(\mathrm{d}, \mathrm{p}) / / \omega \mathrm{B} 97 \mathrm{X}- \\
\mathrm{D} / \mathbf{6 - 3 1 G ( d )} \\
\end{array}$ \\
\hline $5 \mathbf{a}$ & -440.16584496 & 0.122127 & -439.920351 & -440.043718 \\
\hline $6 a$ & -270.53433179 & 0.096333 & -270.362697 & -270.4379988 \\
\hline $4 e$ & -2017.01238872 & 0.504297 & -2015.898700 & -2016.508092 \\
\hline
\end{tabular}




\begin{tabular}{|c|c|c|c|c|}
\hline $\mathrm{BF}_{4}^{-}$ & -424.635472 & -0.010638 & -424.397143 & -424.64611 \\
\hline $4 e^{\prime} B F_{4}$ & -2441.685494 & 0.517332 & -2440.454165 & -2441.168162 \\
\hline $4 e \cdot 5 a$ & -2457.20890196 & 0.650746 & -2455.836810 & -2456.558156 \\
\hline $4 e_{c y-H} \cdot 5 a$ & -2457.20426314 & 0.654273 & -2455.830871 & -2456.54999 \\
\hline IM1' & -2727.759526 & 0.770015 & -2726.201862 & -2726.989511 \\
\hline TS1' & -2727.74901860 & 0.767979 & -2726.194263 & -2726.98104 \\
\hline IM2' & -2727.752005 & 0.770516 & -2726.195417 & -2726.981489 \\
\hline TS2' & -2727.714576 & 0.766446 & -2726.167481 & -2726.94813 \\
\hline IM3' & -2727.766046 & 0.773760 & -2726.214796 & -2726.992286 \\
\hline IM4' & -2727.778329 & 0.772685 & -2726.228235 & -2727.005644 \\
\hline IM1 & -2727.763833 & 0.771895 & -2726.203905 & -2726.991938 \\
\hline TS1 & -2727.73670798 & 0.765916 & -2726.180459 & -2726.970792 \\
\hline IM2 & -2727.778499 & 0.777986 & -2726.221834 & -2727.000513 \\
\hline IM3 & -2727.793929 & 0.780322 & -2726.239885 & -2727.013607 \\
\hline
\end{tabular}

Structure 5a

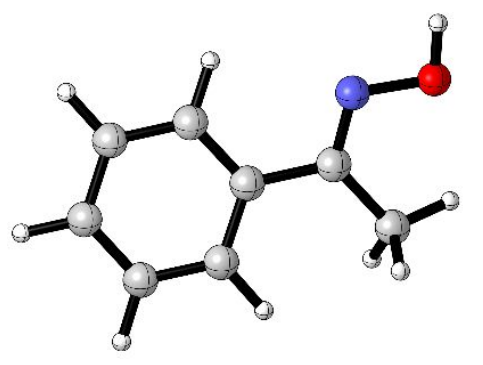

Zero-point correction=

0.156966 (Hartree/Particle)

Thermal correction to Energy=

0.165977

Thermal correction to Enthalpy=

0.166921

Thermal correction to Gibbs Free Energy=

0.122127

Sum of electronic and zero-point Energies=

$-439.885513$

Sum of electronic and thermal Energies=

$-439.876502$

Sum of electronic and thermal Enthalpies=

$-439.875557$

Sum of electronic and thermal Free Energies=

$-439.920351$

Number of Imaginary Frequencies $=0$

E (Single Point Energy) $\left[\operatorname{IEFPCM}_{(\mathrm{DCM})} \omega \mathrm{B} 97 \mathrm{X}-\mathrm{D} / 6-311++\mathrm{G}(\mathrm{d}, \mathrm{p})\right]=-440.16584496$

$\begin{array}{lrrr}01 & & & \\ \text { C } & -3.00724600 & -0.20665900 & -0.02548600 \\ \text { C } & -2.19494300 & -1.33175500 & 0.10923500 \\ \text { C } & -0.81376500 & -1.20091400 & 0.13114200 \\ \text { C } & -0.21402100 & 0.06057000 & 0.01710600 \\ \text { C } & -1.03760200 & 1.18249700 & -0.11582800 \\ \text { C } & -2.42339400 & 1.04960700 & -0.13869600 \\ \text { H } & -4.08809200 & -0.31081600 & -0.03965400 \\ \text { H } & -2.64217000 & -2.31708600 & 0.20266700 \\ \text { H } & -0.18007300 & -2.07420000 & 0.23912900\end{array}$




$\begin{array}{lrrr}\mathrm{H} & -0.60199400 & 2.17211300 & -0.21226500 \\ \mathrm{H} & -3.04538100 & 1.93333200 & -0.24628400 \\ \mathrm{C} & 1.26605600 & 0.20451200 & 0.03110700 \\ \mathrm{C} & 1.88514600 & 1.56139000 & 0.22510400 \\ \mathrm{H} & 1.38067800 & 2.10062100 & 1.03224800 \\ \mathrm{H} & 2.94441800 & 1.47045400 & 0.46211000 \\ \mathrm{H} & 1.78970300 & 2.16018600 & -0.68827400 \\ \mathrm{~N} & 1.93657100 & -0.87520600 & -0.13809400 \\ \mathrm{O} & 3.30929500 & -0.67003500 & -0.11792700 \\ \mathrm{H} & 3.65116100 & -1.56336400 & -0.24169500\end{array}$

Structure 6a

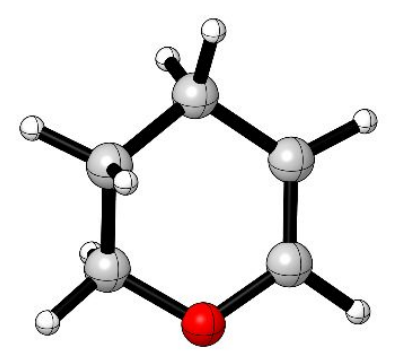

Zero-point correction $=$

0.124694 (Hartree/Particle)

Thermal correction to Energy=

0.129848

Thermal correction to Enthalpy=

0.130792

Thermal correction to Gibbs Free Energy=

Sum of electronic and zero-point Energies=

0.096333

Sum of electronic and thermal Energies=

$-270.334335$

$-270.329182$

Sum of electronic and thermal Enthalpies=

$-270.328237$

Sum of electronic and thermal Free Energies=

$-270.362697$

Number of Imaginary Frequencies $=0$

E (Single Point Energy) $\left[\operatorname{IEFPCM}_{(\mathrm{DCM})} \omega \mathrm{B} 97 \mathrm{X}-\mathrm{D} / 6-311++\mathrm{G}(\mathrm{d}, \mathrm{p})\right]=-270.53433179$

01

$\begin{array}{lrrr}\mathrm{C} & 0.83821600 & -1.05252300 & -0.32247000 \\ \mathrm{C} & 1.45283100 & 0.27738400 & 0.12625800 \\ \mathrm{C} & 0.40545200 & 1.35727400 & 0.06208100 \\ \mathrm{C} & -0.89110000 & 1.07572700 & -0.08166700 \\ \mathrm{C} & -0.54760000 & -1.20731600 & 0.29296100 \\ \mathrm{H} & 0.74055400 & -1.06980600 & -1.41397200 \\ \mathrm{H} & 1.85701000 & 0.18613600 & 1.14418900 \\ \mathrm{H} & -0.47480400 & -1.19220900 & 1.39022500 \\ \mathrm{O} & -1.43193500 & -0.16947200 & -0.11491000 \\ \mathrm{H} & -1.02836900 & -2.13950500 & -0.01165400\end{array}$




$\begin{array}{rrrc}\mathrm{H} & 1.46521100 & -1.90224100 & -0.03231600 \\ \mathrm{H} & 2.30187300 & 0.53609900 & -0.51765000 \\ \mathrm{H} & -1.65848200 & 1.83466200 & -0.19518600 \\ \mathrm{H} & 0.70569000 & 2.39936500 & 0.09266900\end{array}$

Structure 4e

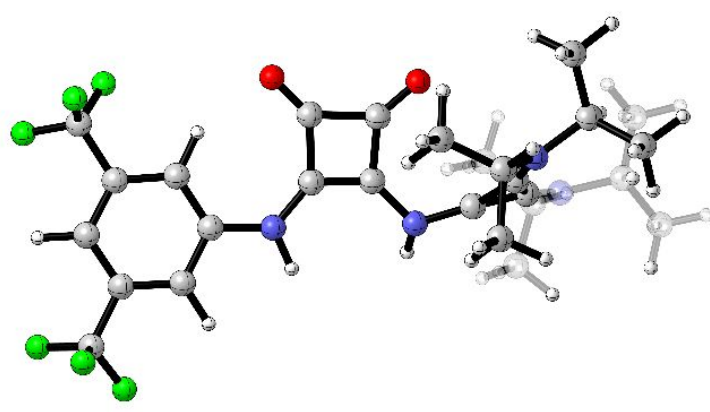

Zero-point correction=

0.584726 (Hartree/Particle)

Thermal correction to Energy=

0.625644

Thermal correction to Enthalpy=

0.626588

Thermal correction to Gibbs Free Energy=

0.504297

Sum of electronic and zero-point Energies=

$-2015.818271$

Sum of electronic and thermal Energies=

$-2015.777352$

Sum of electronic and thermal Enthalpies=

$-2015.776408$

Sum of electronic and thermal Free Energies=

$-2015.898700$

Number of Imaginary Frequencies $=0$

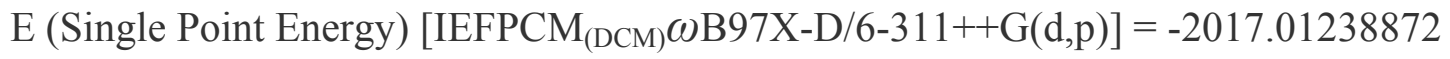

$\begin{array}{lrrr}11 & & & \\ \mathrm{C} & 0.85281800 & -0.16587000 & -0.31150100 \\ \mathrm{C} & -0.51705500 & 0.01747100 & -0.27976100 \\ \mathrm{C} & -0.84042200 & -1.22031600 & -0.99150600 \\ \mathrm{C} & 0.68050000 & -1.47977700 & -1.07204400 \\ \mathrm{O} & 1.40147400 & -2.30525500 & -1.54846900 \\ \mathrm{O} & -1.85701600 & -1.77407700 & -1.32977700 \\ \mathrm{~N} & -1.35089200 & 1.01353200 & 0.21617600 \\ \mathrm{H} & -1.13289600 & 1.97051700 & -0.03701800 \\ \mathrm{C} & -2.68819100 & 0.70936100 & 0.29432200 \\ \mathrm{C} & -3.97715300 & 0.89489800 & -0.14011900 \\ \mathrm{C} & -3.65111500 & -0.15900800 & 0.74914800 \\ \mathrm{~N} & -4.03795200 & -1.21092400 & 1.42793700 \\ \mathrm{~N} & -4.84984700 & 1.54743900 & -0.87099800 \\ \mathrm{C} & -4.35250600 & 2.48873300 & -1.90387800 \\ \mathrm{C} & -3.75824200 & 3.73955400 & -1.26092700\end{array}$




\begin{tabular}{|c|c|c|c|}
\hline $\mathrm{C}$ & -3.38725000 & 1.78981100 & -2.85932400 \\
\hline $\mathrm{H}$ & -2.87541700 & 3.49542200 & -0.65766500 \\
\hline $\mathrm{H}$ & -3.44463600 & 4.44715900 & -2.03336600 \\
\hline $\mathrm{H}$ & -4.48462700 & 4.23556600 & -0.61178300 \\
\hline $\mathrm{H}$ & -2.45990400 & 1.49507400 & -2.35540300 \\
\hline $\mathrm{H}$ & -3.83912700 & 0.89100600 & -3.28773100 \\
\hline $\mathrm{H}$ & -3.11991000 & 2.46604600 & -3.67595600 \\
\hline $\mathrm{C}$ & -6.30099400 & 1.28556300 & -0.72536700 \\
\hline $\mathrm{C}$ & -6.83063100 & 0.49291300 & -1.91797400 \\
\hline $\mathrm{H}$ & -6.75955900 & 1.07028300 & -2.84582100 \\
\hline $\mathrm{H}$ & -6.27172500 & -0.43890800 & -2.05194100 \\
\hline $\mathrm{H}$ & -7.88494900 & 0.24577400 & -1.76409600 \\
\hline $\mathrm{C}$ & -5.33858600 & -1.85000000 & 1.11350900 \\
\hline $\mathrm{C}$ & -5.12980400 & -3.30036900 & 0.68188200 \\
\hline $\mathrm{C}$ & -6.32849300 & -1.69241400 & 2.26421200 \\
\hline $\mathrm{H}$ & -4.76935000 & -3.92545900 & 1.50501700 \\
\hline $\mathrm{H}$ & -6.08266500 & -3.71963800 & 0.34639800 \\
\hline $\mathrm{H}$ & -4.41409500 & -3.35775600 & -0.14310900 \\
\hline $\mathrm{H}$ & -7.29943300 & -2.10510200 & 1.97548500 \\
\hline $\mathrm{H}$ & -5.99894600 & -2.22824000 & 3.16021500 \\
\hline $\mathrm{H}$ & -6.46486700 & -0.63801800 & 2.52595700 \\
\hline $\mathrm{C}$ & -3.17733300 & -1.77332600 & 2.49922400 \\
\hline $\mathrm{C}$ & -1.91705400 & -2.43969800 & 1.95636000 \\
\hline $\mathrm{H}$ & -1.19722700 & -1.69028400 & 1.61244000 \\
\hline $\mathrm{H}$ & -1.43579600 & -3.01029000 & 2.75586300 \\
\hline $\mathrm{H}$ & -2.14046100 & -3.11615200 & 1.12862800 \\
\hline $\mathrm{N}$ & 1.85227900 & 0.56989600 & 0.19063400 \\
\hline $\mathrm{H}$ & 1.57386300 & 1.34763800 & 0.77582200 \\
\hline $\mathrm{H}$ & -5.72040900 & -1.30662300 & 0.24488000 \\
\hline $\mathrm{H}$ & -5.23710000 & 2.78242000 & -2.47347100 \\
\hline $\mathrm{H}$ & -3.79485900 & -2.54115100 & 2.97049200 \\
\hline $\mathrm{C}$ & -2.86607700 & -0.70445100 & 3.54379600 \\
\hline $\mathrm{H}$ & -3.78344800 & -0.26307500 & 3.94415700 \\
\hline $\mathrm{H}$ & -2.30683300 & -1.14800500 & 4.37227300 \\
\hline $\mathrm{H}$ & -2.24834100 & 0.09519300 & 3.11830900 \\
\hline $\mathrm{H}$ & -6.39018000 & 0.67359300 & 0.17715300 \\
\hline $\mathrm{C}$ & -7.07308500 & 2.57961500 & -0.47620100 \\
\hline $\mathrm{H}$ & -6.66859500 & 3.12011500 & 0.38433600 \\
\hline $\mathrm{H}$ & -7.05854000 & 3.24353300 & -1.34624900 \\
\hline $\mathrm{H}$ & -8.11971200 & 2.34022500 & -0.26851900 \\
\hline $\mathrm{C}$ & 3.25432900 & 0.39229700 & 0.09025200 \\
\hline $\mathrm{C}$ & 3.83547700 & -0.59133800 & -0.70367400 \\
\hline $\mathrm{C}$ & 4.05364200 & 1.27321500 & 0.81723000 \\
\hline $\mathrm{C}$ & 5.22266100 & -0.68272100 & -0.75303200 \\
\hline $\mathrm{H}$ & 3.23079000 & -1.28696400 & -1.27502000 \\
\hline $\mathrm{C}$ & 5.43551000 & 1.16592700 & 0.75047900 \\
\hline
\end{tabular}




$\begin{array}{lrrr}\mathrm{H} & 3.60731100 & 2.04969600 & 1.43186500 \\ \mathrm{C} & 6.03312300 & 0.18705700 & -0.03509300 \\ \mathrm{H} & 7.11205400 & 0.11125100 & -0.09451300 \\ \mathrm{C} & 6.28896600 & 2.08907600 & 1.58619600 \\ \mathrm{C} & 5.84128000 & -1.79014000 & -1.57483100 \\ \mathrm{~F} & 6.51443400 & 1.56423800 & 2.79928300 \\ \mathrm{~F} & 7.47577300 & 2.31360200 & 1.01328900 \\ \mathrm{~F} & 5.68636200 & 3.27584500 & 1.77061600 \\ \mathrm{~F} & 7.10442000 & -1.50455700 & -1.91341200 \\ \mathrm{~F} & 5.85334600 & -2.94085000 & -0.88717000 \\ \mathrm{~F} & 5.14820800 & -2.00229800 & -2.70218200\end{array}$

Structure $\mathbf{B F}_{4}^{-}$

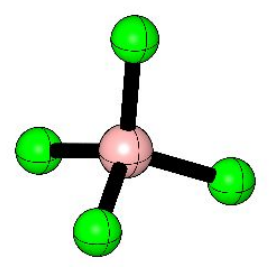

Zero-point correction=

Thermal correction to Energy=

0.014624 (Hartree/Particle)

Thermal correction to Enthalpy=

0.018974

Thermal correction to Gibbs Free Energy=

0.019919

Sum of electronic and zero-point Energies=

$-0.010638$

Sum of electronic and thermal Energies $=$

$-424.371881$

$-424.367531$

Sum of electronic and thermal Enthalpies=

$-424.366586$

Sum of electronic and thermal Free Energies=

$-424.397143$

Number of Imaginary Frequencies $=0$

E (Single Point Energy) $\left[\mathrm{IEFPCM}_{(\mathrm{DCM})} \omega \mathrm{B} 97 \mathrm{X}-\mathrm{D} / 6-311++\mathrm{G}(\mathrm{d}, \mathrm{p})\right]=-424.635472$

$-11$

B $\quad 0.00000000 \quad 0.00000000 \quad 0.00000000$

$\begin{array}{llll}\text { F } & 0.81341700 & 0.81341700 & 0.81341700\end{array}$

F $\quad-0.81341700 \quad-0.81341700 \quad 0.81341700$

F $\quad-0.81341700 \quad 0.81341700 \quad-0.81341700$

F $\quad 0.81341700 \quad-0.81341700 \quad-0.81341700$

Structure $\mathbf{4 e} \cdot \mathbf{B F}_{4}$ 


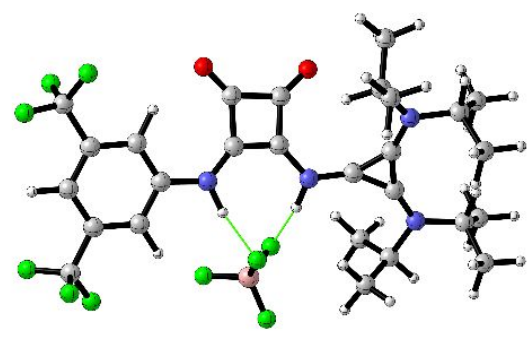

Zero-point correction=

Thermal correction to Energy=

Thermal correction to Enthalpy=

Thermal correction to Gibbs Free Energy=

Sum of electronic and zero-point Energies=

Sum of electronic and thermal Energies=

Sum of electronic and thermal Enthalpies=

Sum of electronic and thermal Free Energies=
0.602446 (Hartree/Particle)

0.648609

0.649553

0.517332

$-2440.369051$

$-2440.322888$

$-2440.321944$

$-2440.454165$

Number of Imaginary Frequencies $=0$

E (Single Point Energy) $\left[\mathrm{IEFPCM}_{(\mathrm{DCM})} \omega \mathrm{B} 97 \mathrm{X}-\mathrm{D} / 6-311++\mathrm{G}(\mathrm{d}, \mathrm{p})\right]=-2441.685494$

01

$\begin{array}{lrrr}\mathrm{O} & 1.50907700 & 2.91186400 & -1.72144100 \\ \mathrm{O} & -1.73545500 & 3.22795900 & -1.28672900 \\ \mathrm{~N} & 4.70489100 & -1.53022200 & -0.28964100 \\ \mathrm{~N} & 4.18028600 & 2.08586300 & 0.60627700 \\ \mathrm{~N} & 1.39688800 & -0.09984300 & -0.44962000 \\ \mathrm{H} & 1.07275200 & -1.06782800 & -0.60489200 \\ \mathrm{~N} & -1.75154700 & 0.07580800 & -0.04377900 \\ \mathrm{H} & -1.33235200 & -0.79430900 & 0.30655800 \\ \mathrm{C} & 2.70422900 & 0.10907100 & -0.23067800 \\ \mathrm{C} & 3.96440300 & -0.44116400 & -0.15040100 \\ \mathrm{C} & 3.75351800 & 0.90524500 & 0.17732200 \\ \mathrm{C} & 4.01854100 & -2.80038900 & -0.64404500 \\ \mathrm{H} & 4.81811500 & -3.54420300 & -0.69604700 \\ \mathrm{C} & 3.06310100 & -3.22254500 & 0.46914300 \\ \mathrm{H} & 2.30708600 & -2.46404800 & 0.68501500 \\ \mathrm{H} & 3.61966900 & -3.41480400 & 1.39223900 \\ \mathrm{H} & 2.51460800 & -4.12347600 & 0.19031100 \\ \mathrm{C} & 3.38662100 & -2.71214500 & -2.03080900 \\ \mathrm{H} & 4.14809400 & -2.47889400 & -2.78188700 \\ \mathrm{H} & 2.59939600 & -1.95842200 & -2.08263200 \\ \mathrm{H} & 2.91837700 & -3.66668900 & -2.28294000 \\ \mathrm{C} & 6.15547000 & -1.52908000 & -0.04916900\end{array}$




\begin{tabular}{|c|c|c|c|}
\hline $\mathrm{H}$ & 6.41469700 & -0.49934700 & 0.19559700 \\
\hline $\mathrm{C}$ & 6.52505000 & -2.40167300 & 1.15023200 \\
\hline $\mathrm{H}$ & 7.59578500 & -2.31226600 & 1.35822500 \\
\hline $\mathrm{H}$ & 6.30962400 & -3.45887700 & 0.96489800 \\
\hline $\mathrm{H}$ & 5.97110100 & -2.09252500 & 2.04209200 \\
\hline $\mathrm{C}$ & 6.92295800 & -1.90504700 & -1.31674500 \\
\hline $\mathrm{H}$ & 6.65113900 & -1.24264700 & -2.14423300 \\
\hline $\mathrm{H}$ & 6.72330200 & -2.93684500 & -1.62331800 \\
\hline $\mathrm{H}$ & 7.99892000 & -1.81481100 & -1.13850100 \\
\hline $\mathrm{C}$ & 5.60766900 & 2.32610200 & 0.87738400 \\
\hline $\mathrm{H}$ & 5.66240900 & 3.37303200 & 1.18492700 \\
\hline $\mathrm{C}$ & 6.10774100 & 1.48959600 & 2.05811700 \\
\hline $\mathrm{H}$ & 5.94464800 & 0.41921900 & 1.90842800 \\
\hline $\mathrm{H}$ & 5.58183000 & 1.77409900 & 2.97383900 \\
\hline $\mathrm{H}$ & 7.17907500 & 1.65057500 & 2.21270700 \\
\hline $\mathrm{C}$ & 6.43303700 & 2.20115600 & -0.40369200 \\
\hline $\mathrm{H}$ & 6.09423000 & 2.93751300 & -1.13789700 \\
\hline $\mathrm{H}$ & 6.34175700 & 1.21305000 & -0.86434800 \\
\hline $\mathrm{H}$ & 7.49195400 & 2.37951900 & -0.19369800 \\
\hline $\mathrm{C}$ & 3.19100400 & 3.13209300 & 0.95132600 \\
\hline $\mathrm{H}$ & 2.22684300 & 2.72832200 & 0.64247700 \\
\hline $\mathrm{C}$ & 3.43321800 & 4.41295300 & 0.15868200 \\
\hline $\mathrm{H}$ & 3.45523700 & 4.19863400 & -0.91095000 \\
\hline $\mathrm{H}$ & 4.36300900 & 4.91090500 & 0.45659500 \\
\hline $\mathrm{H}$ & 2.61208500 & 5.11152500 & 0.34288800 \\
\hline $\mathrm{C}$ & 3.15063000 & 3.36702400 & 2.46131100 \\
\hline $\mathrm{H}$ & 4.08910500 & 3.79578500 & 2.83085300 \\
\hline $\mathrm{H}$ & 2.95700700 & 2.43442400 & 3.00037100 \\
\hline $\mathrm{H}$ & 2.35098600 & 4.07439700 & 2.70055900 \\
\hline $\mathrm{C}$ & 0.49221300 & 0.91595800 & -0.66752500 \\
\hline $\mathrm{C}$ & 0.60923500 & 2.23540000 & -1.27502800 \\
\hline $\mathrm{C}$ & -0.90911300 & 2.38054300 & -1.08152800 \\
\hline $\mathrm{C}$ & -0.89111800 & 0.97128100 & -0.52189800 \\
\hline $\mathrm{C}$ & -3.15836300 & 0.13626100 & 0.02776400 \\
\hline $\mathrm{C}$ & -3.87735000 & 1.31817900 & -0.12778600 \\
\hline $\mathrm{H}$ & -3.37509000 & 2.25348900 & -0.35014100 \\
\hline $\mathrm{C}$ & -5.26394800 & 1.28589700 & -0.01105500 \\
\hline $\mathrm{C}$ & -5.93792600 & 0.10475400 & 0.26618500 \\
\hline $\mathrm{H}$ & -7.01706300 & 0.09379700 & 0.35947700 \\
\hline $\mathrm{C}$ & -5.20252200 & -1.06723500 & 0.41585500 \\
\hline $\mathrm{C}$ & -3.82117300 & -1.06516700 & 0.29297900 \\
\hline $\mathrm{H}$ & -3.25503500 & -1.98860600 & 0.36279100 \\
\hline $\mathrm{C}$ & -5.91862900 & -2.34505700 & 0.76944500 \\
\hline $\mathrm{C}$ & -6.03941300 & 2.55635400 & -0.24480900 \\
\hline $\mathrm{F}$ & -1.70299500 & -3.16552000 & -0.64008700 \\
\hline $\mathrm{F}$ & 0.43771500 & -2.52806100 & -1.2034900 \\
\hline
\end{tabular}




$\begin{array}{lrcc}\text { F } & 0.10303200 & -4.28767900 & 0.24901500 \\ \text { F } & -0.35866800 & -2.11583500 & 0.88819800 \\ \text { B } & -0.40264100 & -3.08514200 & -0.17677500 \\ \text { F } & -5.38335200 & 3.63091100 & 0.22077100 \\ \text { F } & -6.25678000 & 2.76268400 & -1.55441100 \\ \text { F } & -7.24098900 & 2.52519500 & 0.35564800 \\ \text { F } & -6.10555300 & -2.44230500 & 2.09857500 \\ \text { F } & -7.13508200 & -2.40073700 & 0.19951300 \\ \text { F } & -5.23701300 & -3.42909600 & 0.38224200\end{array}$

Structure 4e.5a

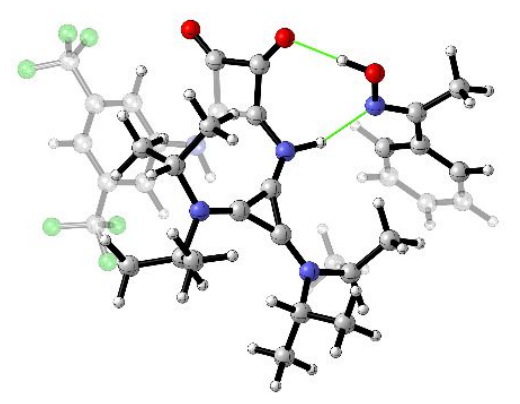

Zero-point correction $=$

0.744064 (Hartree/Particle)

Thermal correction to Energy=

0.794992

Thermal correction to Enthalpy=

0.795936

Thermal correction to Gibbs Free Energy=

0.650746

Sum of electronic and zero-point Energies=

$-2455.743491$

Sum of electronic and thermal Energies $=$

$-2455.692564$

Sum of electronic and thermal Enthalpies=

$-2455.691619$

Sum of electronic and thermal Free Energies=

$-2455.836810$

Number of Imaginary Frequencies $=0$

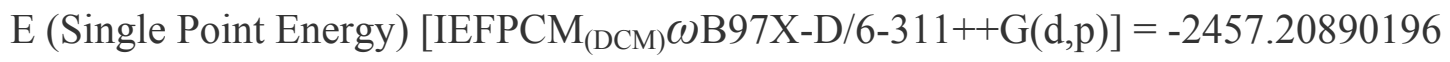

$\begin{array}{lrrr}11 & & & \\ \mathrm{C} & -0.84173800 & -1.36139300 & -0.86063100 \\ \mathrm{C} & 0.48435800 & -1.29537900 & -1.24539700 \\ \mathrm{C} & 0.34870000 & -2.24123200 & -2.34948000 \\ \mathrm{C} & -1.16169900 & -2.30120000 & -2.01786300 \\ \mathrm{O} & -2.14429100 & -2.79397900 & -2.48583900 \\ \mathrm{O} & 1.08396700 & -2.73825800 & -3.16929700 \\ \mathrm{~N} & 1.58201700 & -0.61750600 & -0.74684700 \\ \mathrm{C} & 1.62588100 & 0.72677300 & -0.63866700 \\ \mathrm{C} & 2.30065900 & 1.82763700 & -0.15353300 \\ \mathrm{C} & 1.14500100 & 1.98771800 & -0.93687500\end{array}$




\begin{tabular}{|c|c|c|c|}
\hline $\mathrm{N}$ & 0.28032800 & 2.80842300 & -1.51450200 \\
\hline $\mathrm{N}$ & 3.31746000 & 2.32410800 & 0.51980100 \\
\hline $\mathrm{C}$ & 4.21206000 & 1.40069600 & 1.26079400 \\
\hline $\mathrm{C}$ & 3.41962300 & 0.51939900 & 2.22037700 \\
\hline $\mathrm{C}$ & 5.08869800 & 0.60211200 & 0.29951300 \\
\hline $\mathrm{H}$ & 2.77038800 & -0.18300700 & 1.68807700 \\
\hline $\mathrm{H}$ & 4.10613500 & -0.08325400 & 2.82065100 \\
\hline $\mathrm{H}$ & 2.80886400 & 1.12788300 & 2.89469600 \\
\hline $\mathrm{H}$ & 4.48890600 & -0.05523800 & -0.33868700 \\
\hline $\mathrm{H}$ & 5.67641500 & 1.26328000 & -0.34296300 \\
\hline $\mathrm{H}$ & 5.77399000 & -0.03347900 & 0.86690200 \\
\hline $\mathrm{C}$ & 3.58239000 & 3.77949000 & 0.52880300 \\
\hline $\mathrm{C}$ & 4.99294600 & 4.09608800 & 0.03482300 \\
\hline $\mathrm{H}$ & 5.76286600 & 3.72230400 & 0.71695400 \\
\hline $\mathrm{H}$ & 5.16730700 & 3.66873700 & -0.95682000 \\
\hline $\mathrm{H}$ & 5.11612600 & 5.18070900 & -0.03321300 \\
\hline $\mathrm{C}$ & 0.33378000 & 4.24892800 & -1.16854600 \\
\hline $\mathrm{C}$ & 1.13997500 & 5.03694900 & -2.20099500 \\
\hline $\mathrm{C}$ & -1.06330100 & 4.82550700 & -0.94924700 \\
\hline $\mathrm{H}$ & 0.63877000 & 5.05304900 & -3.17420900 \\
\hline $\mathrm{H}$ & 1.25689800 & 6.07458600 & -1.87452000 \\
\hline $\mathrm{H}$ & 2.13653600 & 4.60471800 & -2.34158500 \\
\hline $\mathrm{H}$ & -0.97389600 & 5.85892300 & -0.60266900 \\
\hline $\mathrm{H}$ & -1.65430100 & 4.84123100 & -1.87012100 \\
\hline $\mathrm{H}$ & -1.60972600 & 4.25720100 & -0.19146900 \\
\hline $\mathrm{C}$ & -0.53693700 & 2.37529800 & -2.67693900 \\
\hline $\mathrm{C}$ & 0.26753400 & 1.49967700 & -3.63464900 \\
\hline $\mathrm{H}$ & 0.51694900 & 0.52794400 & -3.19739500 \\
\hline $\mathrm{H}$ & -0.32224800 & 1.30688700 & -4.53475500 \\
\hline $\mathrm{H}$ & 1.19658400 & 1.99386500 & -3.93431500 \\
\hline $\mathrm{N}$ & -1.47164000 & -0.78682700 & 0.17131000 \\
\hline $\mathrm{H}$ & -0.87021700 & -0.31648900 & 0.83685700 \\
\hline $\mathrm{H}$ & 0.84917900 & 4.29186700 & -0.20435700 \\
\hline $\mathrm{H}$ & 4.85620800 & 2.05106900 & 1.85760200 \\
\hline $\mathrm{H}$ & -0.76927500 & 3.30253200 & -3.20744400 \\
\hline $\mathrm{C}$ & -1.85237300 & 1.73376100 & -2.24629000 \\
\hline $\mathrm{H}$ & -2.43018700 & 2.39700300 & -1.59769100 \\
\hline $\mathrm{H}$ & -2.45976200 & 1.49896200 & -3.12526100 \\
\hline $\mathrm{H}$ & -1.67125800 & 0.80409100 & -1.70510500 \\
\hline $\mathrm{H}$ & 2.88430000 & 4.20499200 & -0.19475700 \\
\hline $\mathrm{C}$ & 3.28416000 & 4.37574600 & 1.90296400 \\
\hline $\mathrm{H}$ & 2.25466100 & 4.16243900 & 2.20785700 \\
\hline $\mathrm{H}$ & 3.95780500 & 3.97781300 & 2.66903400 \\
\hline $\mathrm{H}$ & 3.41923300 & 5.46091300 & 1.87726300 \\
\hline $\mathrm{C}$ & -2.85486300 & -0.68513900 & 0.44551000 \\
\hline$C$ & -3.82822300 & -1.17630200 & -0.41937700 \\
\hline
\end{tabular}




$\begin{array}{lrrr}\mathrm{C} & -3.22146400 & -0.02392800 & 1.61616200 \\ \mathrm{C} & -5.16858500 & -0.99934900 & -0.09220100 \\ \mathrm{H} & -3.56060700 & -1.69797900 & -1.33236000 \\ \mathrm{C} & -4.56483900 & 0.14331800 & 1.92152200 \\ \mathrm{H} & -2.46561300 & 0.36816000 & 2.29052100 \\ \mathrm{C} & -5.55098200 & -0.34180100 & 1.07015200 \\ \mathrm{H} & -6.59910100 & -0.19976300 & 1.30367300 \\ \mathrm{C} & -4.94578200 & 0.81858400 & 3.21664300 \\ \mathrm{C} & -6.21581200 & -1.57633000 & -1.01735700 \\ \mathrm{~F} & -4.03229500 & 1.74187500 & 3.56825000 \\ \mathrm{~F} & -6.13113000 & 1.42958400 & 3.12289000 \\ \mathrm{~F} & -5.01996600 & -0.06792200 & 4.21805000 \\ \mathrm{~F} & -7.40044000 & -0.97455300 & -0.85378100 \\ \mathrm{~F} & -5.85440800 & -1.43468000 & -2.30101700 \\ \mathrm{~F} & -6.38539800 & -2.88435200 & -0.78771500 \\ \mathrm{C} & 4.56944100 & -2.97006600 & -0.47557400 \\ \mathrm{H} & 2.93166800 & -2.57900400 & -2.93299400 \\ \mathrm{O} & 3.83325500 & -2.62107000 & -2.55373900 \\ \mathrm{~N} & 3.60856200 & -2.51786700 & -1.19947600 \\ \mathrm{C} & 4.36853200 & -2.89811100 & 0.99245400 \\ \mathrm{C} & 5.45675500 & -2.70992100 & 1.85094300 \\ \mathrm{C} & 3.09002900 & -3.04996300 & 1.54298600 \\ \mathrm{C} & 5.26913600 & -2.65679800 & 3.22880800 \\ \mathrm{H} & 6.45814200 & -2.60647900 & 1.44218400 \\ \mathrm{C} & 2.90426500 & -3.00084000 & 2.91959500 \\ \mathrm{H} & 2.24960400 & -3.24376500 & 0.88350100 \\ \mathrm{C} & 3.99262300 & -2.80230500 & 3.76656800 \\ \mathrm{H} & 6.12336400 & -2.51506000 & 3.88348100 \\ \mathrm{H} & 1.91153200 & -3.14311400 & 3.33570200 \\ \mathrm{H} & 3.84924500 & -2.77955300 & 4.84227600 \\ \mathrm{C} & 5.82290700 & -3.55006700 & -1.05918800 \\ \mathrm{H} & 5.58073700 & -4.11084800 & -1.96446900 \\ \mathrm{H} & 6.51246600 & -2.75026400 & -1.35452500 \\ \mathrm{H} & 6.32710900 & -4.20280700 & -0.34478400 \\ \mathrm{H} & 2.45925700 & -1.18182800 & -0.75673200\end{array}$

Structure $\mathbf{4} \mathbf{e}_{\mathrm{cy}-\mathrm{H}} \cdot \mathbf{5 a}$ 


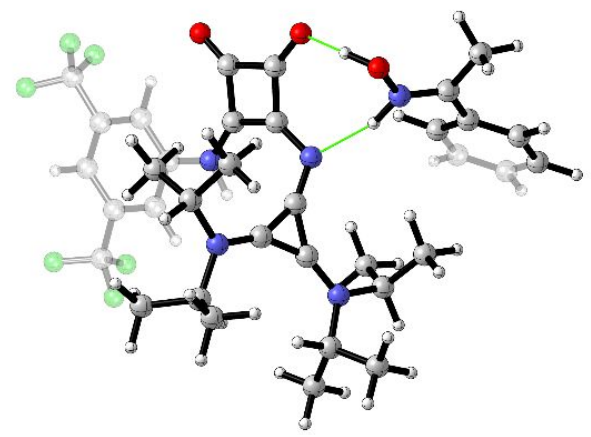

Zero-point correction=

Thermal correction to Energy=

0.743861 (Hartree/Particle)

Thermal correction to Enthalpy=

0.794194

0.795138

Thermal correction to Gibbs Free Energy=

0.654273

Sum of electronic and zero-point Energies=

$-2455.741282$

$-2455.690949$

Sum of electronic and thermal Energies=

$-2455.690005$

Sum of electronic and thermal Enthalpies=

$-2455.830871$

Number of Imaginary Frequencies $=0$

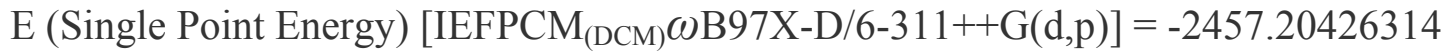

11

$\begin{array}{lrrr}\mathrm{C} & 0.47513200 & -1.80571600 & -0.05994000 \\ \mathrm{C} & -0.84222800 & -1.75058800 & 0.40792900 \\ \mathrm{C} & -0.81783200 & -3.14708200 & 0.83151300 \\ \mathrm{C} & 0.62885300 & -3.23728400 & 0.34765800 \\ \mathrm{O} & 1.49971000 & -4.06642300 & 0.33675400 \\ \mathrm{O} & -1.60914300 & -3.90310800 & 1.37831700 \\ \mathrm{~N} & -1.81413200 & -0.80596100 & 0.43167600 \\ \mathrm{C} & -1.39530700 & 0.44693800 & 0.64465400 \\ \mathrm{C} & -1.60301300 & 1.80919000 & 0.48599800 \\ \mathrm{C} & -0.51772100 & 1.35203900 & 1.24032300 \\ \mathrm{~N} & 0.55727300 & 1.63444100 & 1.97291500 \\ \mathrm{~N} & -2.31257800 & 2.79080000 & -0.04931800 \\ \mathrm{C} & -3.34006600 & 2.44716200 & -1.05404500 \\ \mathrm{C} & -2.72532600 & 1.67994500 & -2.22324000 \\ \mathrm{C} & -4.50669800 & 1.70432200 & -0.40760800 \\ \mathrm{H} & -2.39524800 & 0.68227800 & -1.91166700 \\ \mathrm{H} & -3.47066400 & 1.54878800 & -3.01252000 \\ \mathrm{H} & -1.87282100 & 2.22500400 & -2.64051200 \\ \mathrm{H} & -4.16594800 & 0.75968800 & 0.02908700 \\ \mathrm{H} & -4.96387700 & 2.30690700 & 0.38250400 \\ \mathrm{H} & -5.26951400 & 1.46999800 & -1.15609900 \\ \mathrm{C} & -2.04523000 & 4.19993900 & 0.29546900 \\ \mathrm{C} & -3.30376400 & 4.89557700 & 0.81266600\end{array}$




$\begin{array}{lrrr}\mathrm{H} & -4.06651100 & 4.99919300 & 0.03410900 \\ \mathrm{H} & -3.73627200 & 4.34443800 & 1.65280200 \\ \mathrm{H} & -3.04997700 & 5.90258000 & 1.15615200 \\ \mathrm{C} & 1.03765300 & 3.03459600 & 1.99469100 \\ \mathrm{C} & 0.45570000 & 3.81103100 & 3.17782300 \\ \mathrm{C} & 2.56247400 & 3.10731300 & 1.94711900 \\ \mathrm{H} & 0.85591900 & 3.45082800 & 4.13101000 \\ \mathrm{H} & 0.71220800 & 4.87153400 & 3.09489600 \\ \mathrm{H} & -0.63540900 & 3.72109100 & 3.21559300 \\ \mathrm{H} & 2.87138300 & 4.15478700 & 1.88516000 \\ \mathrm{H} & 3.02135100 & 2.68384200 & 2.84599700 \\ \mathrm{H} & 2.96002300 & 2.58622400 & 1.07268800 \\ \mathrm{C} & 1.03233400 & 0.70338800 & 3.02917800 \\ \mathrm{C} & -0.12624300 & -0.04491800 & 3.68487300 \\ \mathrm{H} & -0.60574800 & -0.74482900 & 2.99269100 \\ \mathrm{H} & 0.25132500 & -0.62785900 & 4.52950700 \\ \mathrm{H} & -0.88506900 & 0.64903100 & 4.05971500 \\ \mathrm{~N} & 1.19852000 & -0.84519900 & -0.66753400 \\ \mathrm{H} & 0.69360700 & 0.01161100 & -0.85303400 \\ \mathrm{H} & 0.66638000 & 3.47748400 & 1.06460700 \\ \mathrm{H} & -3.70602200 & 3.40420900 & -1.43430800 \\ \mathrm{~F} & 1.46564500 & 1.35873600 & 3.79019100 \\ \mathrm{H} & 2.13209200 & -0.24290800 & 2.55218700 \\ \mathrm{C} & 2.89683600 & 0.28078400 & 1.97331900 \\ \mathrm{H} & 2.61492100 & -0.70874800 & 3.41649100 \\ \mathrm{H} & 1.72095100 & -1.04632100 & 1.93728700 \\ \mathrm{H} & -1.33201700 & 4.16361300 & 1.12157700 \\ \mathrm{H} & -1.39623100 & 4.93925100 & -0.87370000 \\ \mathrm{C} & -0.47707700 & 4.43688900 & -1.19262800 \\ \mathrm{H} & -2.07021200 & 4.99929800 & -1.73499500 \\ \mathrm{H} & -1.14477300 & 5.96255000 & -0.57992000 \\ \mathrm{H} & 2.59346900 & -0.74107100 & -0.81691500 \\ \mathrm{H} & 3.45650100 & -1.81371400 & -0.60261200 \\ \mathrm{C} & 3.10169000 & 0.51112900 & -1.15764000 \\ \mathrm{C} & 4.82601600 & -1.60647900 & -0.72282400 \\ \mathrm{C} & 4.07292000 & -2.79666300 & -0.34282600 \\ \mathrm{H} & & & \end{array}$




$\begin{array}{lrrr}\text { F } & 6.00471500 & -2.81341500 & 0.90861600 \\ \text { F } & 5.23952700 & -3.92603900 & -0.78018800 \\ \mathrm{C} & -5.31895300 & -2.31898600 & 0.40706600 \\ \mathrm{H} & -2.98071600 & -3.25101500 & 1.95545700 \\ \mathrm{O} & -3.86779900 & -2.79849700 & 2.11842500 \\ \mathrm{~N} & -4.14634300 & -2.20500400 & 0.93318100 \\ \mathrm{C} & -5.56738300 & -1.66398100 & -0.88573600 \\ \mathrm{C} & -6.83464800 & -1.13560300 & -1.15929600 \\ \mathrm{C} & -4.55994000 & -1.59666300 & -1.85678700 \\ \mathrm{C} & -7.08356500 & -0.52608700 & -2.38235600 \\ \mathrm{H} & -7.62174500 & -1.18712100 & -0.41320900 \\ \mathrm{C} & -4.82265200 & -1.00512800 & -3.08523600 \\ \mathrm{H} & -3.58482600 & -2.03493300 & -1.66702700 \\ \mathrm{C} & -6.07994200 & -0.46511400 & -3.34733200 \\ \mathrm{H} & -8.06452500 & -0.11095200 & -2.58772800 \\ \mathrm{H} & -4.04834300 & -0.97838400 & -3.84479200 \\ \mathrm{H} & -6.28364300 & -0.01009000 & -4.31144300 \\ \mathrm{C} & -6.36419400 & -3.10941700 & 1.11950400 \\ \mathrm{H} & -5.91169100 & -3.98832700 & 1.58395200 \\ \mathrm{H} & -6.79299600 & -2.50814200 & 1.92992800 \\ \mathrm{H} & -7.15765500 & -3.40925600 & 0.43557100 \\ \mathrm{H} & -3.36675900 & -1.57570700 & 0.58354200\end{array}$

Structure IM1'

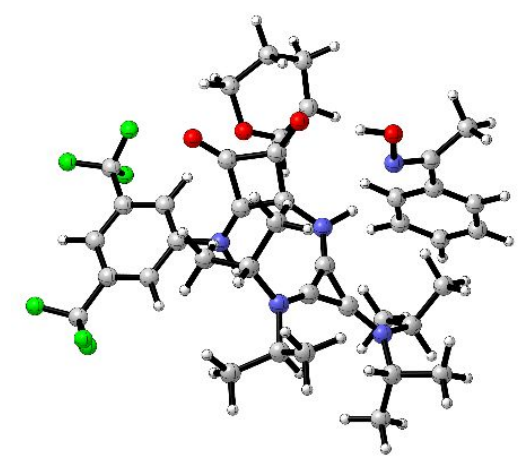

Zero-point correction=

0.870187 (Hartree/Particle)

Thermal correction to Energy=

0.927889

Thermal correction to Enthalpy=

0.928834

0.770015

Thermal correction to Gibbs Free Energy=

$-2726.101691$

Sum of electronic and zero-point Energies=

$-2726.043988$

Sum of electronic and thermal Energies=

$-2726.043044$

Sum of electronic and thermal Enthalpies=

$-2726.201862$

Number of Imaginary Frequencies $=0$ 
E (Single Point Energy) $\left[\operatorname{IEFPCM}_{(\mathrm{DCM})} \omega \mathrm{B} 97 \mathrm{X}-\mathrm{D} / 6-311++\mathrm{G}(\mathrm{d}, \mathrm{p})\right]=-2727.759526$

\begin{tabular}{|c|c|c|c|}
\hline & & & \\
\hline $\mathrm{C}$ & -0.75692400 & -0.71196500 & -0.57138700 \\
\hline $\mathrm{C}$ & 0.53777500 & -0.75724400 & -1.04096500 \\
\hline $\mathrm{C}$ & 0.26078300 & -1.74165200 & -2.08053100 \\
\hline $\mathrm{C}$ & -1.23258900 & -1.62332100 & -1.68542600 \\
\hline $\mathrm{O}$ & -2.29259400 & -1.95299600 & -2.12896400 \\
\hline $\mathrm{O}$ & 0.90662700 & -2.34633600 & -2.90760200 \\
\hline $\mathrm{N}$ & 1.69249500 & -0.09836800 & -0.65483100 \\
\hline $\mathrm{C}$ & 1.72331700 & 1.24076000 & -0.54467600 \\
\hline $\mathrm{C}$ & 2.37531500 & 2.36988700 & -0.08783800 \\
\hline $\mathrm{C}$ & 1.17054700 & 2.48109400 & -0.80096200 \\
\hline $\mathrm{N}$ & 0.22581700 & 3.26115200 & -1.31722700 \\
\hline $\mathrm{N}$ & 3.40623900 & 2.89523800 & 0.54066200 \\
\hline $\mathrm{C}$ & 4.38006000 & 1.99181900 & 1.20177300 \\
\hline $\mathrm{C}$ & 3.69184500 & 1.11750100 & 2.24345200 \\
\hline $\mathrm{C}$ & 5.17031100 & 1.18669000 & 0.17282700 \\
\hline $\mathrm{H}$ & 2.98650500 & 0.41889600 & 1.78207500 \\
\hline $\mathrm{H}$ & 4.43465700 & 0.51240300 & 2.76975600 \\
\hline $\mathrm{H}$ & 3.15694900 & 1.72998700 & 2.97596300 \\
\hline $\mathrm{H}$ & 4.52601500 & 0.50038300 & -0.38668600 \\
\hline $\mathrm{H}$ & 5.67542500 & 1.84428300 & -0.54019400 \\
\hline $\mathrm{H}$ & 5.92278900 & 0.57782400 & 0.68160200 \\
\hline $\mathrm{C}$ & 3.61477200 & 4.35842800 & 0.56846300 \\
\hline $\mathrm{C}$ & 4.94550400 & 4.74270800 & -0.07598400 \\
\hline $\mathrm{H}$ & 5.80137100 & 4.37606200 & 0.49955300 \\
\hline $\mathrm{H}$ & 5.01623900 & 4.34826400 & -1.09395800 \\
\hline $\mathrm{H}$ & 5.02596800 & 5.83248400 & -0.12339500 \\
\hline $\mathrm{C}$ & 0.22925300 & 4.69358500 & -0.93375800 \\
\hline $\mathrm{C}$ & 1.01938100 & 5.54245300 & -1.93186900 \\
\hline $\mathrm{C}$ & -1.18871500 & 5.22052600 & -0.72223300 \\
\hline $\mathrm{H}$ & 0.51014700 & 5.60594200 & -2.89869400 \\
\hline $\mathrm{H}$ & 1.12851800 & 6.56283400 & -1.55226100 \\
\hline $\mathrm{H}$ & 2.01926300 & 5.13002000 & -2.10495000 \\
\hline $\mathrm{H}$ & -1.13771400 & 6.24587800 & -0.34509000 \\
\hline $\mathrm{H}$ & -1.76180000 & 5.24503800 & -1.65416200 \\
\hline $\mathrm{H}$ & -1.73796000 & 4.61751400 & 0.00621000 \\
\hline $\mathrm{C}$ & -0.53820600 & 2.85427500 & -2.52951000 \\
\hline $\mathrm{C}$ & 0.30072500 & 1.98643900 & -3.46420600 \\
\hline $\mathrm{H}$ & 0.49839200 & 0.99625300 & -3.04267500 \\
\hline $\mathrm{H}$ & -0.24331600 & 1.83415700 & -4.40007600 \\
\hline $\mathrm{H}$ & 1.25664100 & 2.46429700 & -3.69991600 \\
\hline $\mathrm{N}$ & -1.33258200 & -0.05594300 & 0.43706200 \\
\hline $\mathrm{H}$ & -0.74502800 & 0.54038500 & 1.00505100 \\
\hline
\end{tabular}




\begin{tabular}{|c|c|c|c|}
\hline $\mathrm{H}$ & 0.73227500 & 4.72640600 & 0.03820300 \\
\hline $\mathrm{H}$ & 5.07398300 & 2.65651500 & 1.72187100 \\
\hline $\mathrm{H}$ & -0.72963500 & 3.79530000 & -3.05287900 \\
\hline $\mathrm{C}$ & -1.88694000 & 2.22241900 & -2.19998500 \\
\hline $\mathrm{H}$ & -2.48804100 & 2.85816300 & -1.54517100 \\
\hline $\mathrm{H}$ & -2.45099200 & 2.06032800 & -3.12324800 \\
\hline $\mathrm{H}$ & -1.76235600 & 1.25166300 & -1.71796300 \\
\hline $\mathrm{H}$ & 2.81828000 & 4.77551800 & -0.05031000 \\
\hline $\mathrm{C}$ & 3.45746200 & 4.90453300 & 1.98627800 \\
\hline $\mathrm{H}$ & 2.48216300 & 4.63503000 & 2.40356900 \\
\hline $\mathrm{H}$ & 4.23503200 & 4.52339900 & 2.65624800 \\
\hline $\mathrm{H}$ & 3.53907600 & 5.99525900 & 1.97562700 \\
\hline $\mathrm{C}$ & -2.73504600 & 0.02718400 & 0.65038800 \\
\hline $\mathrm{C}$ & -3.52647100 & -1.11641300 & 0.60797000 \\
\hline $\mathrm{C}$ & -3.29281300 & 1.27562700 & 0.87855500 \\
\hline $\mathrm{C}$ & -4.89892200 & -0.98064200 & 0.75593300 \\
\hline $\mathrm{H}$ & -3.06470500 & -2.08826600 & 0.47625000 \\
\hline $\mathrm{C}$ & -4.66945200 & 1.39190800 & 1.03772100 \\
\hline $\mathrm{H}$ & -2.66172900 & 2.15868300 & 0.90069400 \\
\hline $\mathrm{C}$ & -5.48115200 & 0.26813600 & 0.96987500 \\
\hline $\mathrm{H}$ & -6.55561700 & 0.35976500 & 1.08105600 \\
\hline $\mathrm{C}$ & -5.24151300 & 2.76374500 & 1.28569000 \\
\hline $\mathrm{C}$ & -5.79303800 & -2.19326000 & 0.65288100 \\
\hline $\mathrm{F}$ & -5.03643600 & 3.15478500 & 2.55199500 \\
\hline $\mathrm{F}$ & -4.63965500 & 3.67755500 & 0.49391300 \\
\hline $\mathrm{F}$ & -6.55288000 & 2.81479300 & 1.04545000 \\
\hline $\mathrm{F}$ & -6.68484000 & -2.21916700 & 1.65281400 \\
\hline $\mathrm{F}$ & -6.47985100 & -2.18095600 & -0.49709400 \\
\hline $\mathrm{F}$ & -5.09039800 & -3.33439800 & 0.69759200 \\
\hline $\mathrm{C}$ & 4.64551300 & -2.49477500 & -0.52589700 \\
\hline $\mathrm{H}$ & 2.75473900 & -2.22995900 & -2.81500400 \\
\hline $\mathrm{O}$ & 3.69375300 & -2.26651700 & -2.53507000 \\
\hline $\mathrm{N}$ & 3.62157500 & -2.07104300 & -1.17596400 \\
\hline $\mathrm{C}$ & 4.58662500 & -2.34118900 & 0.94890900 \\
\hline $\mathrm{C}$ & 5.75448200 & -2.17631100 & 1.70018100 \\
\hline $\mathrm{C}$ & 3.35641700 & -2.40785100 & 1.61483400 \\
\hline $\mathrm{C}$ & 5.69317400 & -2.07543900 & 3.08720600 \\
\hline $\mathrm{H}$ & 6.71898300 & -2.13214200 & 1.20192400 \\
\hline $\mathrm{C}$ & 3.29759600 & -2.31770000 & 3.00020500 \\
\hline $\mathrm{H}$ & 2.45385700 & -2.56647300 & 1.03294900 \\
\hline $\mathrm{C}$ & 4.46588500 & -2.15178100 & 3.74133400 \\
\hline $\mathrm{H}$ & 6.60804300 & -1.95307200 & 3.65858200 \\
\hline $\mathrm{H}$ & 2.33994200 & -2.39731900 & 3.50631400 \\
\hline $\mathrm{H}$ & 4.42186700 & -2.09587400 & 4.82448900 \\
\hline $\mathrm{C}$ & 5.82669200 & -3.13749600 & -1.18943500 \\
\hline $\mathrm{H}$ & 5.49742900 & -3.68694200 & -2.0734680 \\
\hline
\end{tabular}




$\begin{array}{lrrr}\mathrm{H} & 6.53619200 & -2.37397800 & -1.53025800 \\ \mathrm{H} & 6.34473900 & -3.81181500 & -0.50496300 \\ \mathrm{C} & 0.77940300 & -4.67025400 & 0.21811300 \\ \mathrm{C} & 0.40364100 & -5.57390200 & -0.92387200 \\ \mathrm{C} & -0.06854300 & -3.77914400 & 0.73249400 \\ \mathrm{H} & 1.76992600 & -4.74908200 & 0.65460800 \\ \mathrm{C} & -1.89317200 & -4.67698100 & -0.45452500 \\ \mathrm{H} & 0.34343700 & -6.61085600 & -0.56802100 \\ \mathrm{H} & 1.19052000 & -5.55577200 & -1.68628100 \\ \mathrm{H} & 0.18309900 & -3.10253600 & 1.54459100 \\ \mathrm{O} & -1.35390600 & -3.59257500 & 0.31729600 \\ \mathrm{C} & -0.93024000 & -5.13587100 & -1.54004400 \\ \mathrm{H} & -2.11879600 & -5.49717800 & 0.23863400 \\ \mathrm{H} & -2.82673600 & -4.29638500 & -0.87134300 \\ \mathrm{H} & -1.39173200 & -5.95535300 & -2.09960200 \\ \mathrm{H} & -0.76792500 & -4.31956800 & -2.25020600 \\ \mathrm{H} & 2.55586200 & -0.67888900 & -0.69214900\end{array}$

Structure TS1'

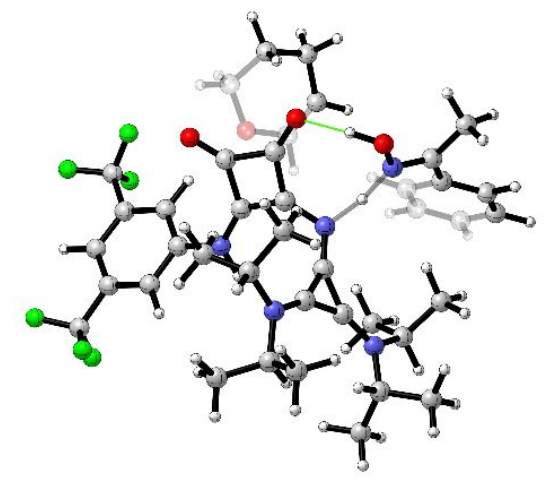

Zero-point correction=

0.865773 (Hartree/Particle)

Thermal correction to Energy=

0.922840

Thermal correction to Enthalpy=

0.923784

Thermal correction to Gibbs Free Energy=

0.767979

Sum of electronic and zero-point Energies=

$-2726.096469$

Sum of electronic and thermal Energies=

$-2726.039403$

Sum of electronic and thermal Enthalpies=

$-2726.038458$

Sum of electronic and thermal Free Energies=

$-2726.194263$

Number of Imaginary Frequencies $=1$

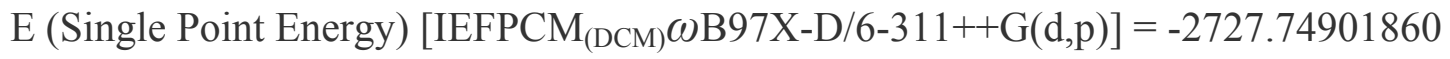

11

C $\quad 0.65074800 \quad-0.78205600 \quad 0.61880000$ 


\begin{tabular}{|c|c|c|c|}
\hline $\mathrm{C}$ & -0.65840900 & -0.78108400 & 1.07254600 \\
\hline $\mathrm{C}$ & -0.42121500 & -1.83226900 & 2.05206100 \\
\hline $\mathrm{C}$ & 1.06684500 & -1.77924400 & 1.66651500 \\
\hline $\mathrm{O}$ & 2.10255900 & -2.21811300 & 2.08133400 \\
\hline $\mathrm{O}$ & -1.10729200 & -2.43494500 & 2.86019400 \\
\hline $\mathrm{N}$ & -1.79239900 & -0.09233800 & 0.72272100 \\
\hline $\mathrm{C}$ & -1.66819300 & 1.22568500 & 0.54200000 \\
\hline $\mathrm{C}$ & -2.17722700 & 2.40859400 & 0.02393500 \\
\hline $\mathrm{C}$ & -0.99267400 & 2.41966400 & 0.76689800 \\
\hline $\mathrm{N}$ & 0.02225800 & 3.11482300 & 1.28990500 \\
\hline $\mathrm{N}$ & -3.11904300 & 3.01971000 & -0.67504500 \\
\hline $\mathrm{C}$ & -4.17297300 & 2.20165500 & -1.31453400 \\
\hline $\mathrm{C}$ & -3.56692600 & 1.19502500 & -2.28636300 \\
\hline $\mathrm{C}$ & -5.07118700 & 1.55049400 & -0.26496800 \\
\hline $\mathrm{H}$ & -2.94733700 & 0.45956500 & -1.76340200 \\
\hline $\mathrm{H}$ & -4.36131700 & 0.64317000 & -2.79632200 \\
\hline $\mathrm{H}$ & -2.95549400 & 1.70131700 & -3.03971600 \\
\hline $\mathrm{H}$ & -4.50450300 & 0.85282500 & 0.36015200 \\
\hline $\mathrm{H}$ & -5.52536100 & 2.30312000 & 0.38572600 \\
\hline $\mathrm{H}$ & -5.86877300 & 0.98556600 & -0.75607500 \\
\hline $\mathrm{C}$ & -3.15334500 & 4.49040300 & -0.79261900 \\
\hline $\mathrm{C}$ & -4.43228700 & 5.06887300 & -0.18900100 \\
\hline $\mathrm{H}$ & -5.32264900 & 4.76578800 & -0.74958800 \\
\hline $\mathrm{H}$ & -4.55184600 & 4.75059900 & 0.85102600 \\
\hline $\mathrm{H}$ & -4.38757300 & 6.16160100 & -0.21125200 \\
\hline $\mathrm{C}$ & 0.15055000 & 4.53458300 & 0.88557000 \\
\hline $\mathrm{C}$ & -0.66008800 & 5.45965000 & 1.79775100 \\
\hline $\mathrm{C}$ & 1.61225600 & 4.96651000 & 0.78975900 \\
\hline $\mathrm{H}$ & -0.21925100 & 5.52489600 & 2.79753700 \\
\hline $\mathrm{H}$ & -0.68210200 & 6.47240400 & 1.38372600 \\
\hline $\mathrm{H}$ & -1.69233000 & 5.11007900 & 1.90866300 \\
\hline $\mathrm{H}$ & 1.65981500 & 5.98726500 & 0.39959400 \\
\hline $\mathrm{H}$ & 2.10553600 & 4.96862100 & 1.76663200 \\
\hline $\mathrm{H}$ & 2.18472700 & 4.32134400 & 0.11847100 \\
\hline $\mathrm{C}$ & 0.65087000 & 2.69872500 & 2.57575000 \\
\hline $\mathrm{C}$ & -0.31935400 & 1.92624100 & 3.46648100 \\
\hline $\mathrm{H}$ & -0.55668100 & 0.93873400 & 3.06017200 \\
\hline $\mathrm{H}$ & 0.13928600 & 1.77091300 & 4.44672600 \\
\hline $\mathrm{H}$ & -1.25317300 & 2.47859800 & 3.61119300 \\
\hline $\mathrm{N}$ & 1.25646100 & -0.08177800 & -0.34706400 \\
\hline $\mathrm{H}$ & 0.70135500 & 0.61742000 & -0.82261700 \\
\hline $\mathrm{H}$ & -0.26405800 & 4.57959900 & -0.12707000 \\
\hline $\mathrm{H}$ & -4.77679500 & 2.90832600 & -1.88929400 \\
\hline $\mathrm{H}$ & 0.86658500 & 3.64156900 & 3.08686800 \\
\hline $\mathrm{C}$ & 1.97592100 & 1.96557300 & 2.38795200 \\
\hline $\mathrm{H}$ & 2.66039500 & 2.51919100 & 1.74049800 \\
\hline
\end{tabular}




\begin{tabular}{|c|c|c|c|}
\hline $\mathrm{H}$ & 2.46145200 & 1.83638600 & 3.36001000 \\
\hline $\mathrm{H}$ & 1.82769900 & 0.97130300 & 1.96305200 \\
\hline $\mathrm{H}$ & -2.31581500 & 4.84820400 & -0.19253100 \\
\hline $\mathrm{C}$ & -2.92462200 & 4.93091000 & -2.23775500 \\
\hline $\mathrm{H}$ & -1.98785600 & 4.52035800 & -2.62727300 \\
\hline $\mathrm{H}$ & -3.74001300 & 4.61073700 & -2.89472100 \\
\hline $\mathrm{H}$ & -2.87122600 & 6.02226800 & -2.29000800 \\
\hline $\mathrm{C}$ & 2.65482300 & -0.03480600 & -0.56753900 \\
\hline $\mathrm{C}$ & 3.41844000 & -1.19897900 & -0.55455100 \\
\hline $\mathrm{C}$ & 3.24515800 & 1.20030500 & -0.78615200 \\
\hline $\mathrm{C}$ & 4.79119200 & -1.09795600 & -0.72064800 \\
\hline $\mathrm{H}$ & 2.93430300 & -2.16028700 & -0.43439000 \\
\hline $\mathrm{C}$ & 4.62257700 & 1.28180400 & -0.96257700 \\
\hline $\mathrm{H}$ & 2.63692900 & 2.10026600 & -0.78773400 \\
\hline $\mathrm{C}$ & 5.40569300 & 0.13755200 & -0.92375100 \\
\hline $\mathrm{H}$ & 6.47985200 & 0.20193000 & -1.05249800 \\
\hline $\mathrm{C}$ & 5.22092300 & 2.64779900 & -1.17155900 \\
\hline $\mathrm{C}$ & 5.65019500 & -2.33741600 & -0.64833000 \\
\hline $\mathrm{F}$ & 4.82492000 & 3.17769000 & -2.33966400 \\
\hline $\mathrm{F}$ & 4.80763600 & 3.49565200 & -0.20427100 \\
\hline $\mathrm{F}$ & 6.55421800 & 2.62972000 & -1.15568400 \\
\hline $\mathrm{F}$ & 6.56480500 & -2.34792900 & -1.62829000 \\
\hline $\mathrm{F}$ & 6.31000900 & -2.39334400 & 0.51624200 \\
\hline $\mathrm{F}$ & 4.91904700 & -3.45674700 & -0.75669500 \\
\hline $\mathrm{C}$ & -4.74071500 & -2.10442300 & 0.73324800 \\
\hline $\mathrm{H}$ & -2.77733700 & -1.92696800 & 2.93283200 \\
\hline $\mathrm{O}$ & -3.72254100 & -1.70085300 & 2.72193900 \\
\hline $\mathrm{N}$ & -3.72780400 & -1.61562000 & 1.35781100 \\
\hline $\mathrm{C}$ & -4.70425800 & -2.06231500 & -0.74238700 \\
\hline $\mathrm{C}$ & -5.88006100 & -1.87799700 & -1.47772400 \\
\hline $\mathrm{C}$ & -3.49602700 & -2.28253100 & -1.41378400 \\
\hline $\mathrm{C}$ & -5.84297000 & -1.89671600 & -2.86716300 \\
\hline $\mathrm{H}$ & -6.82451500 & -1.71891500 & -0.96492400 \\
\hline $\mathrm{C}$ & -3.46728400 & -2.31887400 & -2.80274800 \\
\hline $\mathrm{H}$ & -2.59161400 & -2.47363600 & -0.84405700 \\
\hline $\mathrm{C}$ & -4.63830500 & -2.12382600 & -3.53090600 \\
\hline $\mathrm{H}$ & -6.75768300 & -1.75152200 & -3.43265500 \\
\hline $\mathrm{H}$ & -2.53226800 & -2.51725000 & -3.31752000 \\
\hline $\mathrm{H}$ & -4.61650100 & -2.16234000 & -4.61537000 \\
\hline $\mathrm{C}$ & -5.88154900 & -2.72102400 & 1.47636700 \\
\hline $\mathrm{H}$ & -5.50400200 & -3.27264600 & 2.34009800 \\
\hline $\mathrm{H}$ & -6.54025500 & -1.93531400 & 1.86470200 \\
\hline $\mathrm{H}$ & -6.45866100 & -3.38182500 & 0.82910600 \\
\hline $\mathrm{C}$ & -0.95008700 & -4.70687400 & -0.58944400 \\
\hline $\mathrm{C}$ & -0.61052800 & -5.72235500 & 0.46752300 \\
\hline $\mathrm{C}$ & -0.08772700 & -3.76207400 & -0.9700950 \\
\hline
\end{tabular}




$\begin{array}{lrcc}\mathrm{H} & -1.92116000 & -4.74285300 & -1.07329900 \\ \mathrm{C} & 1.68740000 & -4.75996800 & 0.21383300 \\ \mathrm{H} & -0.48979900 & -6.71247200 & 0.00830900 \\ \mathrm{H} & -1.43861800 & -5.81381700 & 1.17954800 \\ \mathrm{H} & -0.31487400 & -2.99887200 & -1.70985800 \\ \mathrm{O} & 1.17900800 & -3.62070100 & -0.49740700 \\ \mathrm{C} & 0.66956400 & -5.30972300 & 1.20209200 \\ \mathrm{H} & 1.96019400 & -5.52048100 & -0.52874100 \\ \mathrm{H} & 2.59086000 & -4.40718500 & 0.71232000 \\ \mathrm{H} & 1.11074800 & -6.15913900 & 1.73236500 \\ \mathrm{H} & 0.44566900 & -4.54527500 & 1.95304700 \\ \mathrm{H} & -2.86075400 & -0.84042200 & 0.93013400\end{array}$

Structure IM2'

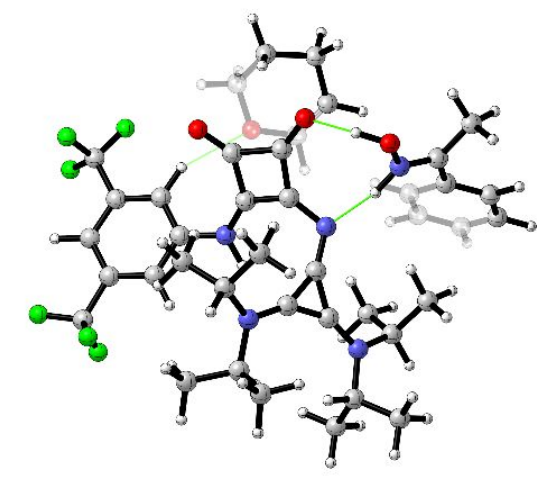

Zero-point correction=

0.869835 (Hartree/Particle)

Thermal correction to Energy=

0.927141

Thermal correction to Enthalpy=

0.928085

0.770516

Thermal correction to Gibbs Free Energy=

$-2726.096099$

Sum of electronic and thermal Energies=

$-2726.038792$

Sum of electronic and thermal Enthalpies=

$-2726.037848$

Sum of electronic and thermal Free Energies=

$-2726.195417$

Number of Imaginary Frequencies $=0$

E (Single Point Energy) $\left[\operatorname{IEFPCM}_{(\mathrm{DCM})} \omega \mathrm{B} 97 \mathrm{X}-\mathrm{D} / 6-311++\mathrm{G}(\mathrm{d}, \mathrm{p})\right]=-2727.752005$

$\begin{array}{lrrr}11 & & & \\ \mathrm{C} & 0.58549300 & -0.80786200 & 0.66869600 \\ \mathrm{C} & -0.73238800 & -0.68464100 & 1.10421800 \\ \mathrm{C} & -0.61258500 & -1.82765600 & 1.99839900 \\ \mathrm{C} & 0.86708700 & -1.91696500 & 1.62926900 \\ \mathrm{O} & 1.83268900 & -2.53312700 & 1.99316100 \\ \mathrm{O} & -1.36436200 & -2.43195200 & 2.75604100\end{array}$




$\begin{array}{lrrr}\mathrm{N} & -1.77212000 & 0.12588900 & 0.75990400 \\ \mathrm{C} & -1.45757800 & 1.40579100 & 0.57267100 \\ \mathrm{C} & -1.76544000 & 2.63007900 & -0.00725500 \\ \mathrm{C} & -0.66707500 & 2.52496700 & 0.84592000 \\ \mathrm{~N} & 0.37316700 & 3.11408100 & 1.44410100 \\ \mathrm{~N} & -2.54363500 & 3.32312100 & -0.82873200 \\ \mathrm{C} & -3.45106200 & 2.57746500 & -1.72482500 \\ \mathrm{C} & -2.66603600 & 1.61366100 & -2.61339300 \\ \mathrm{C} & -4.55741200 & 1.88438200 & -0.93345400 \\ \mathrm{H} & -2.23047900 & 0.80027900 & -2.02156200 \\ \mathrm{H} & -3.33253800 & 1.16285800 & -3.35381600 \\ \mathrm{H} & -1.86463100 & 2.13836500 & -3.14284000 \\ \mathrm{H} & -4.13371400 & 1.15540300 & -0.23532700 \\ \mathrm{H} & -5.14286000 & 2.61178900 & -0.36364700 \\ \mathrm{H} & -5.22746100 & 1.34617000 & -1.61110800 \\ \mathrm{C} & -2.40713600 & 4.78521900 & -0.95512700 \\ \mathrm{C} & -3.74548700 & 5.48785400 & -0.72982200 \\ \mathrm{H} & -4.46536300 & 5.26713100 & -1.52469300 \\ \mathrm{H} & -4.18326800 & 5.19145900 & 0.22777800 \\ \mathrm{H} & -3.59406100 & 6.57116600 & -0.71920400 \\ \mathrm{C} & 0.68234400 & 4.50873600 & 1.05581700 \\ \mathrm{C} & -0.06444000 & 5.51719800 & 1.93306000 \\ \mathrm{C} & 2.18614400 & 4.77544700 & 1.03560400 \\ \mathrm{H} & -1.32120200 & 5.52027700 & 2.95764400 \\ \mathrm{H} & -1.75118700 & 5.17142300 & -2.28046900 \\ \mathrm{H} & -0.77911200 & 4.68021400 & -2.39432000 \\ \mathrm{H} & & & \\ \mathrm{H} & -1.13523000 & 5.29090900 & 1.97843300 \\ \mathrm{H} & 2.36667200 & 5.78640400 & 0.65887300 \\ \mathrm{H} & 2.62750800 & 4.71741200 & 2.03536400 \\ \mathrm{H} & 2.71645000 & 4.07397400 & 0.38627700 \\ \mathrm{C} & 0.88916300 & 2.61552900 & 2.74721200 \\ \mathrm{C} & -0.20509900 & 1.95068000 & 3.57930300 \\ \mathrm{H} & -0.53813400 & 1.00543400 & 3.14062000 \\ \mathrm{H} & 0.18529000 & 1.72784700 & 4.57605700 \\ \mathrm{H} & -1.07288400 & 2.60824800 & 3.69135400 \\ \mathrm{H} & 1.28014500 & -0.11848900 & -0.24507900 \\ \mathrm{H} & 0.82889200 & 0.69066200 & -0.65073600 \\ \mathrm{H} & 0.32455900 & 4.60302600 & 0.02516100 \\ \mathrm{H} & -3.90969800 & 3.33273700 & -2.36802600 \\ \mathrm{H} & 2.19389800 & 3.51860900 & 3.28457200 \\ \mathrm{H} & 1.872597900 & 1.72849200 & 2.59866200 \\ \mathrm{H} & 2.20790400 & 1.99643800 \\ \mathrm{H} & 1.51852600 & 3.58648100 \\ \mathrm{H} & & & \\ \mathrm{H} & & & \end{array}$




\begin{tabular}{|c|c|c|c|}
\hline $\mathrm{H}$ & -2.37741100 & 4.89285900 & -3.13484800 \\
\hline $\mathrm{H}$ & -1.59704300 & 6.25365800 & -2.32379800 \\
\hline$C$ & 2.66791800 & -0.25372500 & -0.48402700 \\
\hline$C$ & 3.25606500 & -1.51043100 & -0.58132800 \\
\hline C & 3.43056700 & 0.89898700 & -0.60967500 \\
\hline $\mathrm{C}$ & 4.62967900 & -1.59413800 & -0.76604800 \\
\hline $\mathrm{H}$ & 2.64046900 & -2.40006100 & -0.51993300 \\
\hline $\mathrm{C}$ & 4.80246100 & 0.79706400 & -0.80506900 \\
\hline $\mathrm{H}$ & 2.95736700 & 1.87254600 & -0.52177600 \\
\hline $\mathrm{C}$ & 5.41374000 & -0.44812100 & -0.87646100 \\
\hline $\mathrm{H}$ & 6.48577400 & -0.52721300 & -1.00988500 \\
\hline $\mathrm{C}$ & 5.59278700 & 2.07372000 & -0.92299200 \\
\hline $\mathrm{C}$ & 5.26023000 & -2.95755800 & -0.91662100 \\
\hline $\mathrm{F}$ & 5.33885100 & 2.69564700 & -2.08543500 \\
\hline $\mathrm{F}$ & 5.25064200 & 2.93571600 & 0.05853600 \\
\hline $\mathrm{F}$ & 6.90855800 & 1.86750700 & -0.84471700 \\
\hline $\mathrm{F}$ & 6.55273600 & -2.94429900 & -0.56857000 \\
\hline $\mathrm{F}$ & 4.63746100 & -3.87192800 & -0.15796300 \\
\hline $\mathrm{F}$ & 5.18722300 & -3.37879000 & -2.18894100 \\
\hline $\mathrm{C}$ & -5.02759100 & -1.81112100 & 0.91795700 \\
\hline $\mathrm{H}$ & -2.85121800 & -1.85023100 & 2.92597200 \\
\hline $\mathrm{O}$ & -3.80644100 & -1.52618200 & 2.83844900 \\
\hline $\mathrm{N}$ & -3.97057400 & -1.36264500 & 1.50519900 \\
\hline $\mathrm{C}$ & -5.15054400 & -1.61404700 & -0.53397700 \\
\hline $\mathrm{C}$ & -6.41452500 & -1.41776500 & -1.10210800 \\
\hline $\mathrm{C}$ & -4.01579600 & -1.65521600 & -1.35421500 \\
\hline $\mathrm{C}$ & -6.53919100 & -1.24596200 & -2.47501900 \\
\hline $\mathrm{H}$ & -7.29879600 & -1.38490800 & -0.47294800 \\
\hline $\mathrm{C}$ & -4.15170400 & -1.50916100 & -2.72810900 \\
\hline $\mathrm{H}$ & -3.03745600 & -1.84165600 & -0.92175900 \\
\hline $\mathrm{C}$ & -5.40939300 & -1.29837800 & -3.28915900 \\
\hline $\mathrm{H}$ & -7.51945800 & -1.08578300 & -2.91122800 \\
\hline $\mathrm{H}$ & -3.27432600 & -1.57027100 & -3.36379100 \\
\hline $\mathrm{H}$ & -5.51199600 & -1.18962000 & -4.36423200 \\
\hline $\mathrm{C}$ & -6.06850500 & -2.51236500 & 1.72406700 \\
\hline $\mathrm{H}$ & -5.59159100 & -3.12375000 & 2.49315900 \\
\hline $\mathrm{H}$ & -6.69079200 & -1.77542100 & 2.24537800 \\
\hline $\mathrm{H}$ & -6.70157400 & -3.13019700 & 1.08767300 \\
\hline $\mathrm{C}$ & -1.67479300 & -4.31298100 & -0.56213300 \\
\hline $\mathrm{C}$ & -1.41477100 & -5.41332600 & 0.43029500 \\
\hline $\mathrm{C}$ & -0.70180000 & -3.52077200 & -1.01839700 \\
\hline $\mathrm{H}$ & -2.67999900 & -4.16827000 & -0.94622600 \\
\hline $\mathrm{C}$ & 0.98245800 & -4.85912500 & -0.06224600 \\
\hline $\mathrm{H}$ & -1.54039500 & -6.38928200 & -0.05715800 \\
\hline $\mathrm{H}$ & -2.15662200 & -5.37463300 & 1.23646300 \\
\hline $\mathrm{H}$ & -0.86639200 & -2.70498900 & -1.7179160 \\
\hline
\end{tabular}




$\begin{array}{lrrr}\mathrm{O} & 0.61002700 & -3.61764100 & -0.68189300 \\ \mathrm{C} & -0.00487000 & -5.27954200 & 1.01667400 \\ \mathrm{H} & 1.04207300 & -5.61817300 & -0.85268100 \\ \mathrm{H} & 1.97906800 & -4.68744900 & 0.34570000 \\ \mathrm{H} & 0.32992800 & -6.22622900 & 1.45175300 \\ \mathrm{H} & 0.00234400 & -4.53928600 & 1.82161300 \\ \mathrm{H} & -3.22665500 & -0.75321700 & 1.05331200\end{array}$

Structure TS2'

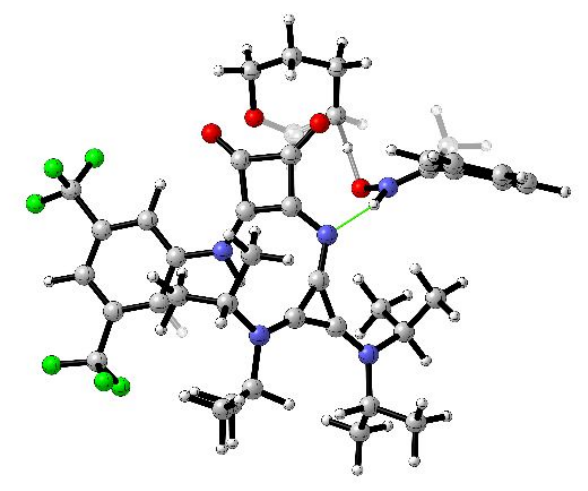

Zero-point correction=

Thermal correction to Energy=

0.865888 (Hartree/Particle)

Thermal correction to Enthalpy=

0.922839

0.923783

0.766446

Thermal correction to Gibbs Free Energy=

$-2726.068040$

Sum of electronic and zero-point Energies=

$-2726.011089$

Sum of electronic and thermal Enthalpies=

$-2726.010145$

Sum of electronic and thermal Free Energies=

$-2726.167481$

Number of Imaginary Frequencies $=1$

E (Single Point Energy) $\left[\operatorname{IEFPCM}_{(\mathrm{DCM})} \omega \mathrm{B} 97 \mathrm{X}-\mathrm{D} / 6-311++\mathrm{G}(\mathrm{d}, \mathrm{p})\right]=-2727.714576$

$\begin{array}{crrr}11 & & & \\ \mathrm{C} & -0.47107100 & 0.97250700 & 0.60516100 \\ \mathrm{C} & 0.86007200 & 0.62729400 & 0.88453200 \\ \mathrm{C} & 1.17516700 & 2.00153000 & 1.30155400 \\ \mathrm{C} & -0.28746900 & 2.36456300 & 1.06011600 \\ \mathrm{O} & -0.96456500 & 3.36832400 & 1.15321300 \\ \mathrm{O} & 2.19117000 & 2.59018500 & 1.61490800 \\ \mathrm{~N} & 1.67457200 & -0.40969300 & 0.67731100 \\ \mathrm{C} & 1.16915100 & -1.63136700 & 0.48606800 \\ \mathrm{C} & 1.31684700 & -2.82692400 & -0.19437200 \\ \mathrm{C} & 0.34606100 & -2.70948000 & 0.80931600 \\ \mathrm{~N} & -0.62186000 & -3.29054400 & 1.51737500 \\ \mathrm{~N} & 1.95561900 & -3.47030800 & -1.15965200\end{array}$




\begin{tabular}{|c|c|c|c|}
\hline $\mathrm{C}$ & 2.99859500 & -2.72876200 & -1.90573200 \\
\hline $\mathrm{C}$ & 2.40317400 & -1.52955500 & -2.64289400 \\
\hline $\mathrm{C}$ & 4.16287900 & -2.35252000 & -0.98925700 \\
\hline $\mathrm{H}$ & 2.01297600 & -0.77469800 & -1.95311200 \\
\hline $\mathrm{H}$ & 3.17397400 & -1.04617000 & -3.25075300 \\
\hline $\mathrm{H}$ & 1.59362700 & -1.84753500 & -3.30735100 \\
\hline $\mathrm{H}$ & 4.93839000 & -1.83241700 & -1.55998200 \\
\hline $\mathrm{H}$ & 3.84080800 & -1.68887200 & -0.18012500 \\
\hline $\mathrm{H}$ & 4.60956800 & -3.24425000 & -0.53969600 \\
\hline $\mathrm{C}$ & 1.65403100 & -4.87074000 & -1.50213600 \\
\hline $\mathrm{C}$ & 1.07784200 & -4.98191900 & -2.91354900 \\
\hline $\mathrm{H}$ & 1.81084600 & -4.69451900 & -3.67468900 \\
\hline $\mathrm{H}$ & 0.78318200 & -6.01607800 & -3.11490400 \\
\hline $\mathrm{C}$ & -1.15746100 & -4.57017000 & 0.98835800 \\
\hline $\mathrm{C}$ & -1.91271100 & -5.39197700 & 2.02783400 \\
\hline $\mathrm{C}$ & -2.00295600 & -4.33209000 & -0.26505800 \\
\hline $\mathrm{H}$ & -2.82784300 & -4.89868000 & 2.36775700 \\
\hline $\mathrm{H}$ & -2.20528700 & -6.33868900 & 1.56516700 \\
\hline $\mathrm{H}$ & -1.29008000 & -5.62754100 & 2.89525900 \\
\hline $\mathrm{H}$ & -2.25811900 & -5.28524500 & -0.73872900 \\
\hline $\mathrm{H}$ & -2.93938800 & -3.81961300 & -0.02221100 \\
\hline $\mathrm{H}$ & -1.45581800 & -3.73190400 & -1.00230900 \\
\hline $\mathrm{C}$ & -1.16164700 & -2.67386800 & 2.75300300 \\
\hline $\mathrm{C}$ & -0.15232400 & -1.73425700 & 3.40581100 \\
\hline $\mathrm{H}$ & -0.02710900 & -0.80674000 & 2.84075800 \\
\hline $\mathrm{H}$ & -0.52075500 & -1.46136700 & 4.39846500 \\
\hline $\mathrm{H}$ & 0.82593600 & -2.20925800 & 3.52440200 \\
\hline $\mathrm{N}$ & -1.43132400 & 0.21479300 & 0.01886300 \\
\hline $\mathrm{H}$ & -1.13493400 & -0.71261100 & -0.25342400 \\
\hline $\mathrm{H}$ & -0.27852500 & -5.16105100 & 0.70803700 \\
\hline $\mathrm{H}$ & 3.37042400 & -3.43263000 & -2.65475800 \\
\hline $\mathrm{H}$ & -1.31293400 & -3.50715300 & 3.44291100 \\
\hline $\mathrm{C}$ & -2.50509700 & -1.98171400 & 2.52825200 \\
\hline $\mathrm{H}$ & -2.37610500 & -1.07194000 & 1.93844300 \\
\hline $\mathrm{H}$ & -3.22638500 & -2.62443700 & 2.01538000 \\
\hline $\mathrm{H}$ & -2.93822800 & -1.69559100 & 3.49165900 \\
\hline $\mathrm{C}$ & 2.86969700 & -5.77141500 & -1.28587200 \\
\hline $\mathrm{H}$ & 2.59384600 & -6.81530200 & -1.46186900 \\
\hline $\mathrm{H}$ & 3.24413800 & -5.68044000 & -0.26207900 \\
\hline $\mathrm{C}$ & -2.80274700 & 0.45516500 & -0.13211300 \\
\hline $\mathrm{C}$ & -3.39747600 & 1.68665500 & 0.14111000 \\
\hline $\mathrm{C}$ & -3.58827600 & -0.61827100 & -0.54776100 \\
\hline $\mathrm{C}$ & -4.77507200 & 1.81241600 & 0.00003800 \\
\hline $\mathrm{H}$ & -2.79650100 & 2.53367400 & 0.46245200 \\
\hline $\mathrm{C}$ & -4.96259900 & -0.47440600 & -0.66524700 \\
\hline $\mathrm{H}$ & -3.12727800 & -1.57963500 & -0.7578370 \\
\hline
\end{tabular}




\begin{tabular}{|c|c|c|c|}
\hline $\mathrm{C}$ & -5.57353700 & 0.74292700 & -0.39476500 \\
\hline $\mathrm{H}$ & -6.64608000 & 0.85716800 & -0.48738000 \\
\hline $\mathrm{C}$ & -5.75532400 & -1.68803500 & -1.07220600 \\
\hline $\mathrm{C}$ & -5.41021200 & 3.13659900 & 0.35121100 \\
\hline $\mathrm{F}$ & -7.07185900 & -1.47433500 & -1.04146100 \\
\hline $\mathrm{F}$ & -5.42869800 & -2.09280400 & -2.31019000 \\
\hline $\mathrm{F}$ & -5.48848800 & -2.72495300 & -0.24671800 \\
\hline $\mathrm{F}$ & -4.65600200 & 4.16486300 & -0.06560900 \\
\hline $\mathrm{F}$ & -6.62332000 & 3.26764600 & -0.20236200 \\
\hline $\mathrm{F}$ & -5.55576100 & 3.26132400 & 1.67916300 \\
\hline $\mathrm{C}$ & 4.77311500 & 1.50165200 & -0.68741500 \\
\hline $\mathrm{H}$ & 2.50791800 & 3.17508500 & -1.46913200 \\
\hline $\mathrm{O}$ & 2.64754100 & 1.84429600 & -1.56354900 \\
\hline $\mathrm{N}$ & 3.50427700 & 1.26589700 & -0.74331200 \\
\hline $\mathrm{C}$ & 5.54442300 & 0.77765000 & 0.33829100 \\
\hline $\mathrm{C}$ & 5.00511800 & 0.60077700 & 1.61924800 \\
\hline $\mathrm{C}$ & 6.79653800 & 0.23357000 & 0.02877000 \\
\hline $\mathrm{C}$ & 5.70922400 & -0.13143400 & 2.56835100 \\
\hline $\mathrm{H}$ & 4.05778500 & 1.06811300 & 1.87531600 \\
\hline $\mathrm{C}$ & 7.48917800 & -0.50199300 & 0.98160000 \\
\hline $\mathrm{H}$ & 7.22221200 & 0.36139500 & -0.96237100 \\
\hline $\mathrm{C}$ & 6.94560600 & -0.68672500 & 2.25140600 \\
\hline $\mathrm{H}$ & 5.29478600 & -0.25534000 & 3.56361900 \\
\hline $\mathrm{H}$ & 8.45530500 & -0.92982600 & 0.73430000 \\
\hline $\mathrm{H}$ & 7.49359200 & -1.25417800 & 2.99699600 \\
\hline $\mathrm{C}$ & 5.36903500 & 2.49439200 & -1.62664800 \\
\hline $\mathrm{H}$ & 6.42964100 & 2.29830000 & -1.78955900 \\
\hline $\mathrm{H}$ & 4.82957800 & 2.48638900 & -2.57732600 \\
\hline $\mathrm{H}$ & 5.28799600 & 3.49958500 & -1.19528600 \\
\hline $\mathrm{C}$ & 2.33579700 & 4.44625900 & -1.75301900 \\
\hline $\mathrm{C}$ & 2.76299000 & 5.39960400 & -0.64992900 \\
\hline $\mathrm{C}$ & 0.96963000 & 4.34325400 & -2.03129900 \\
\hline $\mathrm{H}$ & 2.95377300 & 4.40454500 & -2.65338100 \\
\hline $\mathrm{C}$ & 0.30183100 & 5.79548400 & -0.27269000 \\
\hline $\mathrm{H}$ & 3.01370400 & 6.37424100 & -1.08526000 \\
\hline $\mathrm{H}$ & 3.66690800 & 5.02857300 & -0.15692300 \\
\hline $\mathrm{H}$ & 0.61175900 & 3.73077000 & -2.85766900 \\
\hline $\mathrm{O}$ & 0.00459400 & 4.82215200 & -1.33114000 \\
\hline $\mathrm{C}$ & 1.64282900 & 5.54214700 & 0.38475000 \\
\hline $\mathrm{H}$ & 0.24789000 & 6.76911000 & -0.76763100 \\
\hline $\mathrm{H}$ & -0.52674300 & 5.67733600 & 0.42140800 \\
\hline $\mathrm{H}$ & 1.84699500 & 6.37916900 & 1.05948700 \\
\hline $\mathrm{H}$ & 1.58683300 & 4.64050800 & 0.99905800 \\
\hline $\mathrm{H}$ & 3.06718000 & 0.54279900 & -0.13738200 \\
\hline $\mathrm{H}$ & 3.68419700 & -5.52704700 & -1.97570900 \\
\hline $\mathrm{H}$ & 0.19586700 & -4.34402700 & -3.02644500 \\
\hline
\end{tabular}


Structure IM3,

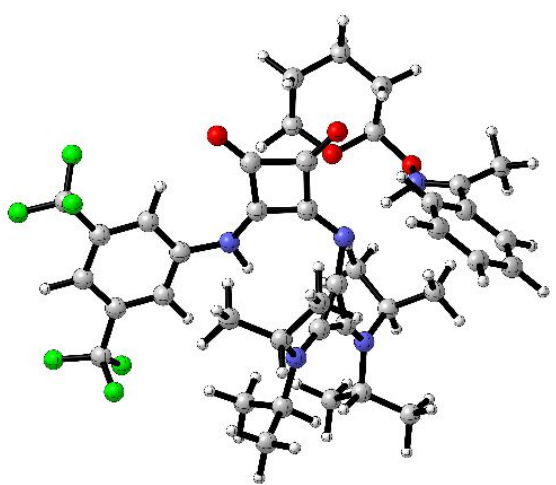

Zero-point correction=

0.871862 (Hartree/Particle)

Thermal correction to Energy=

0.928054

Thermal correction to Enthalpy=

0.928998

Thermal correction to Gibbs Free Energy=

0.773760

Sum of electronic and zero-point Energies=

$-2726.116694$

Sum of electronic and thermal Energies $=$

$-2726.060502$

Sum of electronic and thermal Enthalpies=

$-2726.059558$

Sum of electronic and thermal Free Energies=

$-2726.214796$

Number of Imaginary Frequencies $=0$

E (Single Point Energy) $\left[\operatorname{IEFPCM}_{(\mathrm{DCM})} \omega \mathrm{B} 97 \mathrm{X}-\mathrm{D} / 6-311++\mathrm{G}(\mathrm{d}, \mathrm{p})\right]=-2727.766046$

11

$\begin{array}{lrrr}\mathrm{C} & 0.55552100 & -1.45264100 & 0.22275400 \\ \mathrm{C} & -0.78748200 & -1.19508900 & 0.49679600 \\ \mathrm{C} & -1.00776200 & -2.55388100 & 1.01364500 \\ \mathrm{C} & 0.48172200 & -2.83361000 & 0.76995700 \\ \mathrm{O} & 1.23293800 & -3.76649500 & 0.91388400 \\ \mathrm{O} & -1.97032200 & -3.18518500 & 1.39631200 \\ \mathrm{~N} & -1.65075900 & -0.19336600 & 0.24122300 \\ \mathrm{C} & -1.18128000 & 1.05639600 & 0.17268600 \\ \mathrm{C} & -1.24545400 & 2.29607000 & -0.43923900 \\ \mathrm{C} & -0.40246800 & 2.10194200 & 0.66318400 \\ \mathrm{~N} & 0.47079400 & 2.61105400 & 1.52706600 \\ \mathrm{~N} & -1.74509500 & 3.02861900 & -1.42413600 \\ \mathrm{C} & -2.70936100 & 2.38613500 & -2.34422900 \\ \mathrm{C} & -2.11288000 & 1.14458400 & -3.00621500 \\ \mathrm{C} & -4.02752400 & 2.10568700 & -1.62127400 \\ \mathrm{H} & -1.94844600 & 0.32895900 & -2.29527900 \\ \mathrm{H} & -2.79990400 & 0.77152200 & -3.77140000\end{array}$




\begin{tabular}{|c|c|c|c|}
\hline $\mathrm{H}$ & -1.16265300 & 1.38921500 & -3.49164100 \\
\hline $\mathrm{H}$ & -4.71246100 & 1.56313400 & -2.28071700 \\
\hline $\mathrm{H}$ & -3.85331500 & 1.49707600 & -0.72839700 \\
\hline $\mathrm{H}$ & -4.50792800 & 3.03621800 & -1.30544300 \\
\hline $\mathrm{C}$ & -1.37784500 & 4.44480900 & -1.60335400 \\
\hline $\mathrm{C}$ & -0.60411000 & 4.65176100 & -2.90527700 \\
\hline $\mathrm{H}$ & -1.22628200 & 4.45161000 & -3.78391100 \\
\hline $\mathrm{H}$ & -0.26265100 & 5.68877200 & -2.97424700 \\
\hline $\mathrm{C}$ & 1.10249800 & 3.90601600 & 1.16455700 \\
\hline $\mathrm{C}$ & 1.82279200 & 4.57677400 & 2.32892000 \\
\hline $\mathrm{C}$ & 2.03213400 & 3.74244200 & -0.03930600 \\
\hline $\mathrm{H}$ & 2.68367000 & 3.99585000 & 2.67356800 \\
\hline $\mathrm{H}$ & 2.19930500 & 5.54364800 & 1.98376500 \\
\hline $\mathrm{H}$ & 1.15518100 & 4.76603700 & 3.17397900 \\
\hline $\mathrm{H}$ & 2.36798900 & 4.72097600 & -0.39611500 \\
\hline $\mathrm{H}$ & 2.91964700 & 3.15877300 & 0.22255100 \\
\hline $\mathrm{H}$ & 1.51961700 & 3.24528700 & -0.87181100 \\
\hline $\mathrm{C}$ & 0.84778000 & 1.86174500 & 2.74957000 \\
\hline $\mathrm{C}$ & -0.32570800 & 1.04801700 & 3.29217600 \\
\hline $\mathrm{H}$ & -0.56030200 & 0.18568900 & 2.66258100 \\
\hline $\mathrm{H}$ & -0.05985000 & 0.66649800 & 4.28223200 \\
\hline $\mathrm{H}$ & -1.22647100 & 1.66170800 & 3.39082500 \\
\hline $\mathrm{N}$ & 1.45247000 & -0.68558300 & -0.44858400 \\
\hline $\mathrm{H}$ & 1.07397400 & 0.16831700 & -0.83620700 \\
\hline $\mathrm{H}$ & 0.27551100 & 4.56896500 & 0.88404600 \\
\hline $\mathrm{H}$ & -2.89796900 & 3.12441300 & -3.12775900 \\
\hline $\mathrm{H}$ & 1.06964400 & 2.62865900 & 3.49314200 \\
\hline $\mathrm{C}$ & 2.09749900 & 1.00704200 & 2.55158200 \\
\hline $\mathrm{H}$ & 1.88742200 & 0.16074700 & 1.89452200 \\
\hline $\mathrm{H}$ & 2.92083200 & 1.58193300 & 2.11680200 \\
\hline $\mathrm{H}$ & 2.43363800 & 0.60868000 & 3.51368600 \\
\hline $\mathrm{C}$ & -2.59700000 & 5.35923800 & -1.49059400 \\
\hline $\mathrm{H}$ & -2.28052000 & 6.40518500 & -1.53946300 \\
\hline $\mathrm{H}$ & -3.11766200 & 5.20040100 & -0.54161900 \\
\hline $\mathrm{C}$ & 2.85453800 & -0.73141900 & -0.40056200 \\
\hline $\mathrm{C}$ & 3.55772500 & -1.81748500 & 0.11773100 \\
\hline $\mathrm{C}$ & 3.54472600 & 0.38732400 & -0.86435300 \\
\hline $\mathrm{C}$ & 4.94600300 & -1.75165000 & 0.18053600 \\
\hline $\mathrm{H}$ & 3.03434500 & -2.70673400 & 0.46058500 \\
\hline $\mathrm{C}$ & 4.92821700 & 0.43608900 & -0.77946700 \\
\hline $\mathrm{H}$ & 3.00218300 & 1.23073400 & -1.28123700 \\
\hline $\mathrm{C}$ & 5.64576900 & -0.63175400 & -0.25406700 \\
\hline $\mathrm{H}$ & 6.72590900 & -0.59511700 & -0.18998200 \\
\hline $\mathrm{C}$ & 5.61918800 & 1.71189300 & -1.18114600 \\
\hline $\mathrm{C}$ & 5.68402700 & -2.91798800 & 0.79475700 \\
\hline $\mathrm{F}$ & 6.92218500 & 1.53772600 & -1.4078640 \\
\hline
\end{tabular}




$\begin{array}{lrrr}\text { F } & 5.06958300 & 2.24326400 & -2.28642500 \\ \mathrm{~F} & 5.49737300 & 2.64008000 & -0.20694300 \\ \mathrm{~F} & 6.99540000 & -2.87477600 & 0.52497900 \\ \mathrm{~F} & 5.54395100 & -2.91619400 & 2.12993800 \\ \mathrm{~F} & 5.20797200 & -4.08560700 & 0.34406100 \\ \mathrm{C} & -5.13137100 & -0.86594300 & 0.10444500 \\ \mathrm{H} & -3.60047400 & -3.73402800 & -0.35601900 \\ \mathrm{O} & -4.17439400 & -1.52085600 & -1.86035900 \\ \mathrm{~N} & -4.05273900 & -1.14389300 & -0.55038100 \\ \mathrm{C} & -4.98328600 & -0.31531500 & 1.45724500 \\ \mathrm{C} & -3.99862100 & -0.80425500 & 2.32466000 \\ \mathrm{C} & -5.83282600 & 0.72267600 & 1.86510700 \\ \mathrm{C} & -3.86063500 & -0.24047400 & 3.58697300 \\ \mathrm{H} & -3.37817100 & -1.64653000 & 2.02907600 \\ \mathrm{C} & -5.67097400 & 1.29213800 & 3.12018900 \\ \mathrm{H} & -6.60141200 & 1.09947100 & 1.19631100 \\ \mathrm{C} & -4.68556300 & 0.80985800 & 3.98104500 \\ \mathrm{H} & -3.11328100 & -0.63446700 & 4.26795700 \\ \mathrm{H} & -6.31936200 & 2.10406400 & 3.43220200 \\ \mathrm{H} & -4.57408200 & 1.24472400 & 4.96944400 \\ \mathrm{C} & -6.46418000 & -1.05492900 & -0.54011300 \\ \mathrm{H} & -6.62551000 & -0.27342300 & -1.29184300 \\ \mathrm{H} & -6.49476800 & -2.01169700 & -1.06630300 \\ \mathrm{H} & -7.26055200 & -1.01503700 & 0.20201300 \\ \mathrm{C} & -3.75949200 & -3.91498900 & -1.42505300 \\ \mathrm{C} & -2.85831200 & -5.06705100 & -1.89227000 \\ \mathrm{C} & -3.37865300 & -2.66576700 & -2.19667000 \\ \mathrm{H} & -4.81638800 & -4.14572700 & -1.59671000 \\ \mathrm{C} & -1.14056400 & -3.32910100 & -2.45732700 \\ \mathrm{H} & -3.09143700 & -5.31304800 & -2.93726200 \\ \mathrm{H} & -3.06391600 & -5.96266300 & -1.30054500 \\ \mathrm{H} & -3.60294800 & -2.77233400 & -3.26825100 \\ \mathrm{H} & -2.04512600 & -2.31534200 & -2.00098400 \\ \mathrm{C} & -1.38609600 & -4.66612600 & -1.76798600 \\ \mathrm{H} & -1.25131500 & -3.43112200 & -3.54794100 \\ \mathrm{H} & -0.14255500 & -2.93699400 & -2.25103000 \\ \mathrm{H} & -0.73802200 & -5.42183700 & -2.22346500 \\ \mathrm{H} & -1.11093100 & -4.60075000 & -0.71140500 \\ \mathrm{H} & -3.06695400 & -0.81271400 & -0.23929100 \\ -3.30558500 & 5.19380300 & -2.30901100 \\ & 0.27210500 & 3.99783500 & -2.94825700 \\ \mathrm{H} & -0.70667800 & 4.67505700 & -0.77367100\end{array}$

Structure IM4' 


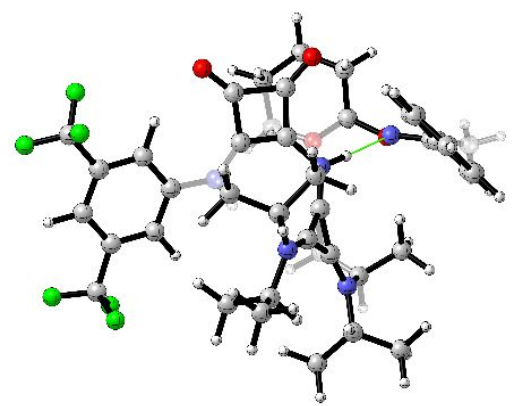

Zero-point correction=

Thermal correction to Energy=

Thermal correction to Enthalpy=

Thermal correction to Gibbs Free Energy=

Sum of electronic and zero-point Energies=

Sum of electronic and thermal Energies=

Sum of electronic and thermal Enthalpies=

Sum of electronic and thermal Free Energies=
0.871805 (Hartree/Particle)

0.928468

0.929413

0.772685

$-2726.129115$

$-2726.072452$

$-2726.071507$

$-2726.228235$

Number of Imaginary Frequencies $=0$

E (Single Point Energy) [IEFPCM(DCM) $\omega$ B97X-D/6-311++G(d,p)] = -2727.778329

$\begin{array}{lrrr}11 & & & \\ \mathrm{C} & 0.63808700 & -1.58926100 & 0.19843900 \\ \mathrm{C} & -0.71537700 & -1.46769200 & 0.43556000 \\ \mathrm{C} & -0.84658600 & -2.79005900 & 1.06727300 \\ \mathrm{C} & 0.69080600 & -2.92885800 & 0.89574000 \\ \mathrm{O} & 1.54213300 & -3.71028300 & 1.21030800 \\ \mathrm{O} & -1.74152300 & -3.47341400 & 1.49006300 \\ \mathrm{~N} & -1.61269400 & -0.47065900 & 0.15250000 \\ \mathrm{C} & -1.27179200 & 0.82840100 & 0.22784700 \\ \mathrm{C} & -1.39801300 & 2.11886300 & -0.24829900 \\ \mathrm{C} & -0.52521700 & 1.82963600 & 0.81934800 \\ \mathrm{~N} & 0.36287400 & 2.26733000 & 1.69890300 \\ \mathrm{~N} & -1.92959200 & 2.96255800 & -1.10702000 \\ \mathrm{C} & -2.68607400 & 2.43474500 & -2.27050700 \\ \mathrm{C} & -1.90456100 & 1.33912200 & -2.99368200 \\ \mathrm{C} & -4.07876500 & 1.97579200 & -1.84736600 \\ \mathrm{H} & -1.90236500 & 0.39295000 & -2.44264900 \\ \mathrm{H} & -2.38038900 & 1.13497300 & -3.95699900 \\ \mathrm{H} & -0.87498500 & 1.65946500 & -3.18808000 \\ \mathrm{H} & -4.60411200 & 1.53203500 & -2.69793700 \\ \mathrm{H} & -4.00494000 & 1.21171500 & -1.06876700 \\ \mathrm{H} & -4.67353200 & 2.80802100 & -1.46148700 \\ \mathrm{C} & -1.73755200 & 4.42062000 & -0.94155600\end{array}$




\begin{tabular}{|c|c|c|c|}
\hline $\mathrm{C}$ & -0.70704300 & 4.95015700 & -1.93652700 \\
\hline $\mathrm{H}$ & -1.05068400 & 4.83024700 & -2.96941300 \\
\hline $\mathrm{H}$ & -0.53397500 & 6.01695500 & -1.76714400 \\
\hline $\mathrm{C}$ & 1.11062000 & 3.50209500 & 1.33729100 \\
\hline $\mathrm{C}$ & 1.75525500 & 4.18254300 & 2.53868200 \\
\hline $\mathrm{C}$ & 2.12477000 & 3.21623900 & 0.22908100 \\
\hline $\mathrm{H}$ & 2.56439300 & 3.58529800 & 2.96971100 \\
\hline $\mathrm{H}$ & 2.19195500 & 5.12729400 & 2.20374500 \\
\hline $\mathrm{H}$ & 1.02452800 & 4.41231600 & 3.31925000 \\
\hline $\mathrm{H}$ & 2.59555400 & 4.14441100 & -0.10788000 \\
\hline $\mathrm{H}$ & 2.91599600 & 2.54163800 & 0.56678900 \\
\hline $\mathrm{H}$ & 1.63285000 & 2.75921200 & -0.63805100 \\
\hline $\mathrm{C}$ & 0.69942800 & 1.47028600 & 2.90632700 \\
\hline $\mathrm{C}$ & -0.49948400 & 0.65204000 & 3.37778100 \\
\hline $\mathrm{H}$ & -0.72119300 & -0.18804200 & 2.71376200 \\
\hline $\mathrm{H}$ & -0.27473500 & 0.23338900 & 4.36263000 \\
\hline $\mathrm{H}$ & -1.39949100 & 1.26735300 & 3.46687600 \\
\hline $\mathrm{N}$ & 1.46511500 & -0.78298200 & -0.49922900 \\
\hline $\mathrm{H}$ & 1.01090700 & -0.03893400 & -1.01337600 \\
\hline $\mathrm{H}$ & 0.36110900 & 4.19404400 & 0.94008200 \\
\hline $\mathrm{H}$ & -2.78032700 & 3.28373900 & -2.95225900 \\
\hline $\mathrm{H}$ & 0.89943800 & 2.21435600 & 3.68009300 \\
\hline $\mathrm{C}$ & 1.94988900 & 0.61213400 & 2.73009900 \\
\hline $\mathrm{H}$ & 1.77538100 & -0.18664200 & 2.00649900 \\
\hline $\mathrm{H}$ & 2.80954100 & 1.19727100 & 2.39243800 \\
\hline $\mathrm{H}$ & 2.21509000 & 0.14729000 & 3.68424000 \\
\hline $\mathrm{C}$ & -3.06496000 & 5.17387300 & -1.00492500 \\
\hline $\mathrm{H}$ & -2.88869400 & 6.22946300 & -0.77985000 \\
\hline $\mathrm{H}$ & -3.77432600 & 4.78163600 & -0.27093000 \\
\hline $\mathrm{C}$ & 2.87092700 & -0.71312600 & -0.47633600 \\
\hline $\mathrm{C}$ & 3.65735200 & -1.59591300 & 0.26162200 \\
\hline $\mathrm{C}$ & 3.46840700 & 0.31728900 & -1.19777900 \\
\hline $\mathrm{C}$ & 5.03514900 & -1.41105300 & 0.28439500 \\
\hline $\mathrm{H}$ & 3.21242600 & -2.42886300 & 0.79804000 \\
\hline $\mathrm{C}$ & 4.84459500 & 0.49182000 & -1.14920700 \\
\hline $\mathrm{H}$ & 2.86444800 & 0.99366300 & -1.79570300 \\
\hline $\mathrm{C}$ & 5.64278100 & -0.36746100 & -0.40540500 \\
\hline $\mathrm{H}$ & 6.71681700 & -0.23381500 & -0.37038700 \\
\hline $\mathrm{C}$ & 5.43353900 & 1.68656800 & -1.85399400 \\
\hline $\mathrm{C}$ & 5.86763200 & -2.33087300 & 1.14746100 \\
\hline $\mathrm{F}$ & 6.75157500 & 1.57652000 & -2.02443700 \\
\hline $\mathrm{F}$ & 4.86818600 & 1.87153600 & -3.05772600 \\
\hline $\mathrm{F}$ & 5.20587300 & 2.80961500 & -1.14151200 \\
\hline $\mathrm{F}$ & 7.15112600 & -2.33601100 & 0.76875400 \\
\hline $\mathrm{F}$ & 5.82392400 & -1.93487000 & 2.43055400 \\
\hline $\mathrm{F}$ & 5.41371400 & -3.58829400 & 1.09991000 \\
\hline
\end{tabular}




$\begin{array}{lrrr}\mathrm{C} & -5.21552000 & -0.80772400 & 0.03437600 \\ \mathrm{H} & -3.33229200 & -3.74378900 & -0.58688300 \\ \mathrm{O} & -4.47199800 & -1.65188000 & -1.89342200 \\ \mathrm{~N} & -4.20477900 & -1.31072600 & -0.57722800 \\ \mathrm{C} & -4.94417800 & -0.34745300 & 1.41782700 \\ \mathrm{C} & -4.07287100 & -1.06516300 & 2.24578300 \\ \mathrm{C} & -5.53521100 & 0.82751700 & 1.89322100 \\ \mathrm{C} & -3.79528700 & -0.60487100 & 3.52742900 \\ \mathrm{H} & -3.62875000 & -1.99130300 & 1.89214200 \\ \mathrm{C} & -5.24443900 & 1.29011400 & 3.17239000 \\ \mathrm{H} & -6.21877300 & 1.38762100 & 1.26076200 \\ \mathrm{C} & -4.37529700 & 0.57406300 & 3.99197000 \\ \mathrm{H} & -3.13541900 & -1.17993900 & 4.16999900 \\ \mathrm{H} & -5.70694100 & 2.20327400 & 3.53380500 \\ \mathrm{H} & -4.16518300 & 0.92460700 & 4.99798100 \\ \mathrm{C} & -6.56290900 & -0.62846100 & -0.59749700 \\ \mathrm{H} & -6.56882600 & 0.27012900 & -1.22656500 \\ \mathrm{H} & -6.78738300 & -1.47602100 & -1.24813900 \\ \mathrm{H} & -7.34034100 & -0.52926700 & 0.16152300 \\ \mathrm{C} & -3.55968300 & -3.91609600 & -1.64492800 \\ \mathrm{C} & -2.51867400 & -4.85505600 & -2.26508300 \\ \mathrm{C} & -3.52815200 & -2.58293100 & -2.37353200 \\ \mathrm{H} & -4.56668600 & -4.33956400 & -1.71399300 \\ \mathrm{C} & -1.24576600 & -2.79559200 & -2.91886000 \\ \mathrm{H} & -2.81650800 & -5.10783300 & -3.29185800 \\ \mathrm{H} & -2.47639700 & -5.79371700 & -1.70622300 \\ \mathrm{H} & -3.82059900 & -2.70493300 & -3.42702900 \\ \mathrm{O} & -2.24902100 & -1.99494300 & -2.30026300 \\ \mathrm{C} & -1.14632700 & -4.17788100 & -2.28341400 \\ \mathrm{H} & -1.46784200 & -2.88713400 & -3.99398300 \\ \mathrm{H} & -0.31418100 & -2.23225500 & -2.81339100 \\ \mathrm{H} & -0.41765000 & -4.77882900 & -2.83752800 \\ \mathrm{H} & -0.77439200 & -4.08440400 & -1.25736600 \\ \mathrm{H} & -3.52216600 & 5.12434900 & -1.99800300 \\ \mathrm{H} & 0.24774600 & 4.42567200 & -1.82941000 \\ \mathrm{H} & -1.34747300 & 4.54341800 & 0.07235100 \\ \mathrm{H} & -2.55641200 & -0.76382000 & -0.17658000\end{array}$

Structure IM1 


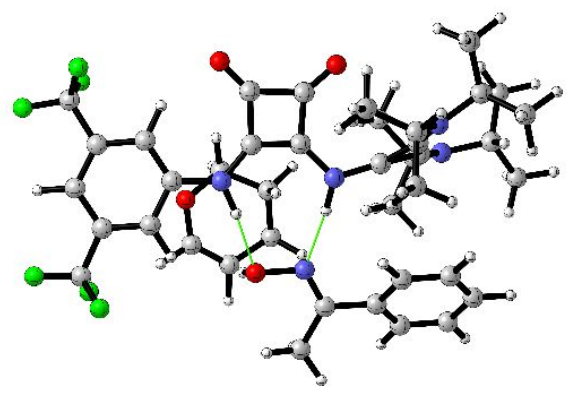

Zero-point correction $=$

0.870262 (Hartree/Particle)

Thermal correction to Energy=

0.927747

Thermal correction to Enthalpy=

0.928691

Thermal correction to Gibbs Free Energy=

0.771895

Sum of electronic and zero-point Energies $=$

$-2726.105538$

Sum of electronic and thermal Energies=

$-2726.048053$

Sum of electronic and thermal Enthalpies=

$-2726.047109$

Sum of electronic and thermal Free Energies=

$-2726.203905$

Number of Imaginary Frequencies $=0$

E (Single Point Energy) $\left[\operatorname{IEFPCM}_{(\mathrm{DCM})} \omega \mathrm{B} 97 \mathrm{X}-\mathrm{D} / 6-311++\mathrm{G}(\mathrm{d}, \mathrm{p})\right]=-2727.763833$

$\begin{array}{lrrr}11 & & & \\ \mathrm{C} & -1.03381400 & -1.49376600 & -0.22431100 \\ \mathrm{C} & 0.34724300 & -1.37526600 & -0.18277200 \\ \mathrm{C} & 0.56280400 & -2.68511600 & 0.42991400 \\ \mathrm{C} & -0.96275900 & -2.87367500 & 0.39261600 \\ \mathrm{O} & -1.74675300 & -3.71261800 & 0.74293400 \\ \mathrm{O} & 1.53785600 & -3.29908300 & 0.80296400 \\ \mathrm{~N} & 1.23356600 & -0.38206200 & -0.49642700 \\ \mathrm{C} & 2.57095300 & -0.62341500 & -0.30629600 \\ \mathrm{C} & 3.70412900 & -0.46126400 & 0.44985400 \\ \mathrm{C} & 3.67860800 & -1.35987900 & -0.65030900 \\ \mathrm{~N} & 4.28500500 & -2.25469300 & -1.38845900 \\ \mathrm{~N} & 4.32729000 & 0.06945600 & 1.47441600 \\ \mathrm{C} & 3.51413300 & 0.69306700 & 2.54703400 \\ \mathrm{C} & 2.78520200 & 1.93104300 & 2.03538500 \\ \mathrm{C} & 2.57418600 & -0.33785100 & 3.16965900 \\ \mathrm{H} & 2.02031800 & 1.66647100 & 1.29747600 \\ \mathrm{H} & 2.28077600 & 2.43170100 & 2.86771200 \\ \mathrm{H} & 3.48145900 & 2.63607400 & 1.57354400 \\ \mathrm{H} & 1.82576900 & -0.68160900 & 2.44725300 \\ \mathrm{H} & 3.12493700 & -1.21240500 & 3.52666200 \\ \mathrm{H} & 2.04660500 & 0.10562800 & 4.01950100 \\ \mathrm{C} & 5.78873000 & -0.09223400 & 1.63284300 \\ \mathrm{C} & 6.11648100 & -1.10965400 & 2.72295400\end{array}$




\begin{tabular}{|c|c|c|c|}
\hline $\mathrm{H}$ & 5.77998000 & -0.76135000 & 3.70522900 \\
\hline $\mathrm{H}$ & 5.63836600 & -2.07364300 & 2.52057100 \\
\hline $\mathrm{H}$ & 7.19803700 & -1.26355000 & 2.77978000 \\
\hline $\mathrm{C}$ & 5.53695500 & -2.88805500 & -0.91567000 \\
\hline $\mathrm{C}$ & 5.32338700 & -4.38298400 & -0.68390500 \\
\hline C & 6.70365600 & -2.57563400 & -1.84840800 \\
\hline $\mathrm{H}$ & 5.14103600 & -4.91923200 & -1.62071000 \\
\hline $\mathrm{H}$ & 6.21977100 & -4.81402900 & -0.22869900 \\
\hline 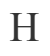 & 4.47510100 & -4.55091100 & -0.01410900 \\
\hline $\mathrm{H}$ & 7.62760500 & -2.99198900 & -1.43687100 \\
\hline $\mathrm{H}$ & 6.56032000 & -3.01531800 & -2.84070000 \\
\hline 11 & 6.83389700 & -1.49493500 & -1.96647700 \\
\hline $\mathrm{C}$ & 3.66108700 & -2.69389100 & -2.66211300 \\
\hline $\mathrm{C}$ & 2.33571000 & -3.41645100 & -2.44107900 \\
\hline $\mathrm{H}$ & 1.55207700 & -2.71346400 & -2.14180300 \\
\hline $\mathrm{H}$ & 2.01642400 & -3.88332500 & -3.37726900 \\
\hline $\mathrm{H}$ & 2.41959000 & -4.18815200 & -1.67286600 \\
\hline $\mathrm{N}$ & -1.96375200 & -0.63377900 & -0.66458800 \\
\hline $\mathrm{H}$ & -1.62289700 & 0.27054300 & -0.99672200 \\
\hline $\mathrm{H}$ & 5.73743400 & -2.43189800 & 0.05751900 \\
\hline $\mathrm{H}$ & 4.23416800 & 1.00626700 & 3.30649800 \\
\hline $\mathrm{H}$ & 4.37365500 & -3.40109000 & -3.09263500 \\
\hline $\mathrm{C}$ & 3.52498400 & -1.50736000 & -3.61350700 \\
\hline $\mathrm{H}$ & 4.48934900 & -1.01598000 & -3.77489500 \\
\hline $\mathrm{H}$ & 3.14414300 & -1.84625900 & -4.58095700 \\
\hline $\mathrm{H}$ & 2.81379200 & -0.77164400 & -3.21920200 \\
\hline $\mathrm{H}$ & 6.13670300 & -0.47628000 & 0.66875600 \\
\hline $\mathrm{C}$ & 6.46576000 & 1.25903800 & 1.85474400 \\
\hline $\mathrm{H}$ & 6.15885300 & 1.97536900 & 1.08750200 \\
\hline $\mathrm{H}$ & 6.23321200 & 1.68073000 & 2.83755900 \\
\hline $\mathrm{H}$ & 7.55113900 & 1.13409000 & 1.80344200 \\
\hline $\mathrm{C}$ & -3.36702200 & -0.70746200 & -0.57973300 \\
\hline $\mathrm{C}$ & -4.04744900 & -1.74822800 & 0.04412100 \\
\hline $\mathrm{C}$ & -4.07758000 & 0.35891000 & -1.13240600 \\
\hline $\mathrm{C}$ & -5.43501000 & -1.68945000 & 0.13384400 \\
\hline $\mathrm{H}$ & -3.51474300 & -2.60171300 & 0.45235300 \\
\hline $\mathrm{C}$ & -5.45768600 & 0.40574000 & -1.01248100 \\
\hline $\mathrm{H}$ & -3.55037200 & 1.15779800 & -1.64521700 \\
\hline $\mathrm{C}$ & -6.15331200 & -0.61629400 & -0.37557000 \\
\hline $\mathrm{H}$ & -7.23178900 & -0.58130200 & -0.28747500 \\
\hline $\mathrm{C}$ & -6.18053600 & 1.62735300 & -1.51336800 \\
\hline $\mathrm{C}$ & -6.14221400 & -2.81041800 & 0.85658800 \\
\hline $\mathrm{F}$ & -7.47596800 & 1.39660300 & -1.73784600 \\
\hline $\mathrm{F}$ & -6.10435300 & 2.63014400 & -0.60767500 \\
\hline $\mathrm{F}$ & -5.63702100 & 2.09501300 & -2.64797800 \\
\hline $\mathrm{F}$ & -5.89837600 & -2.75359700 & 2.1762420 \\
\hline
\end{tabular}




$\begin{array}{lrrr}\text { F } & -7.46975400 & -2.75659700 & 0.68717800 \\ \mathrm{~F} & -5.71993400 & -4.00707700 & 0.42617600 \\ \mathrm{C} & 0.65109800 & 3.41626500 & -1.12304800 \\ \mathrm{H} & -1.63466400 & 2.53831600 & -0.31669500 \\ \mathrm{O} & -1.23628400 & 2.14688600 & -1.13256800 \\ \mathrm{~N} & 0.16027900 & 2.23630100 & -0.99778500 \\ \mathrm{C} & 2.13126300 & 3.49574100 & -1.05461400 \\ \mathrm{C} & 2.75218000 & 4.59212600 & -0.44986900 \\ \mathrm{C} & 2.91999400 & 2.46897200 & -1.58593200 \\ \mathrm{C} & 4.13932400 & 4.64935700 & -0.35696200 \\ \mathrm{H} & 2.15427600 & 5.40141700 & -0.04118800 \\ \mathrm{C} & 4.30457400 & 2.52619500 & -1.49001500 \\ \mathrm{H} & 2.44143100 & 1.64508100 & -2.10636700 \\ \mathrm{C} & 4.91730200 & 3.61696100 & -0.87525900 \\ \mathrm{H} & 4.61274600 & 5.50507000 & 0.11324500 \\ \mathrm{H} & 4.90774600 & 1.73213900 & -1.92109700 \\ \mathrm{H} & 6.00025800 & 3.67490800 & -0.82112400 \\ \mathrm{C} & -0.16612800 & 4.65992900 & -1.30550400 \\ \mathrm{H} & -1.11101500 & 4.42490300 & -1.79803200 \\ \mathrm{H} & -0.39409600 & 5.10776800 & -0.33051900 \\ \mathrm{H} & 0.38281900 & 5.39777400 & -1.89367900 \\ \mathrm{C} & -2.13152900 & 3.25826100 & 1.58156400 \\ \mathrm{C} & -0.87479100 & 2.82266800 & 2.29224800 \\ \mathrm{C} & -3.17137000 & 2.42116900 & 1.41749000 \\ \mathrm{H} & -2.24919000 & 4.28733400 & 1.25614200 \\ \mathrm{C} & -2.18517100 & 0.73338600 & 2.73006300 \\ \mathrm{H} & -0.84995600 & 3.23071900 & 3.31120300 \\ \mathrm{H} & 0.01412400 & 3.21496700 & 1.78263000 \\ \mathrm{H} & -4.08095500 & 2.69424000 & 0.89153500 \\ \mathrm{O} & -3.22355800 & 1.14821900 & 1.84117200 \\ \mathrm{C} & -0.82582200 & 1.29206100 & 2.33014800 \\ \mathrm{H} & -2.45197500 & 1.06246200 & 3.74193300 \\ \mathrm{H} & -2.20396300 & -0.35793200 & 2.70198700 \\ \mathrm{H} & -0.06875100 & 0.93353700 & 3.03372700 \\ \mathrm{H} & -0.55877000 & 0.90497000 & 1.33976500 \\ \mathrm{H} & 0.91175100 & 0.59417100 & -0.61683100\end{array}$

Structure TS1 


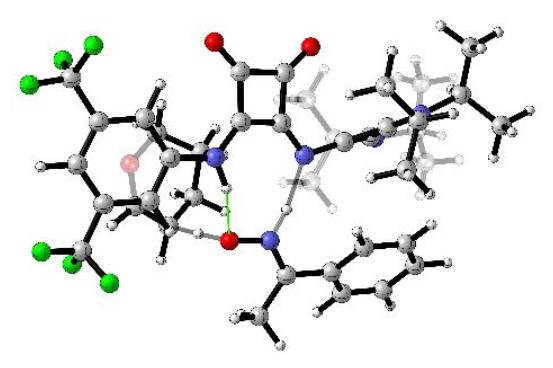

Zero-point correction $=$

0.864768 (Hartree/Particle)

Thermal correction to Energy=

0.921704

Thermal correction to Enthalpy=

0.922648

Thermal correction to Gibbs Free Energy=

0.765916

Sum of electronic and zero-point Energies=

$-2726.081607$

Sum of electronic and thermal Energies=

$-2726.024671$

Sum of electronic and thermal Enthalpies=

$-2726.023727$

Sum of electronic and thermal Free Energies=

$-2726.180459$

Number of Imaginary Frequencies $=1$

E (Single Point Energy) [IEFPCM $\left.{ }_{(\mathrm{DCM})} \omega \mathrm{B} 97 \mathrm{X}-\mathrm{D} / 6-311++\mathrm{G}(\mathrm{d}, \mathrm{p})\right]=-2727.73670798$

$\begin{array}{lrrr}11 & & & \\ \mathrm{C} & -1.06638300 & -1.49728000 & -0.26163500 \\ \mathrm{C} & 0.31305100 & -1.34981100 & -0.11492400 \\ \mathrm{C} & 0.45192700 & -2.68975600 & 0.49941400 \\ \mathrm{C} & -1.06509900 & -2.85516800 & 0.34128900 \\ \mathrm{O} & -1.89031700 & -3.68441000 & 0.64921600 \\ \mathrm{O} & 1.38664600 & -3.31212000 & 0.95120500 \\ \mathrm{~N} & 1.16345200 & -0.32242000 & -0.27714800 \\ \mathrm{C} & 2.47199700 & -0.64642400 & -0.13315300 \\ \mathrm{C} & 3.66273700 & -0.45924800 & 0.53000800 \\ \mathrm{C} & 3.60068600 & -1.30776600 & -0.58464900 \\ \mathrm{~N} & 4.20176300 & -2.11405500 & -1.43834200 \\ \mathrm{~N} & 4.33197300 & 0.08497500 & 1.53501600 \\ \mathrm{C} & 3.54796000 & 0.62853200 & 2.66324600 \\ \mathrm{C} & 2.75210500 & 1.85479100 & 2.22301100 \\ \mathrm{C} & 2.66026100 & -0.44805300 & 3.28860500 \\ \mathrm{H} & 1.98111800 & 1.56802200 & 1.49835800 \\ \mathrm{H} & 2.25587900 & 2.30838800 & 3.08745600 \\ \mathrm{H} & 3.40569200 & 2.60051700 & 1.76156900 \\ \mathrm{H} & 1.90916100 & -0.80689500 & 2.57662700 \\ \mathrm{H} & 3.25095200 & -1.30926500 & 3.61254800 \\ \mathrm{H} & 2.14044900 & -0.04145800 & 4.16231200 \\ \mathrm{C} & 5.79315000 & -0.08073100 & 1.63436600 \\ \mathrm{C} & 6.16454500 & -1.20150400 & 2.60419800 \\ \mathrm{H} & 5.86063000 & -0.95210700 & 3.62671300\end{array}$




\begin{tabular}{|c|c|c|c|}
\hline $\mathrm{H}$ & 5.67764900 & -2.14168600 & 2.32492500 \\
\hline $\mathrm{H}$ & 7.24746600 & -1.35855300 & 2.60891600 \\
\hline 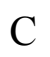 & 5.56100700 & -2.61643100 & -1.15578600 \\
\hline $\mathrm{C}$ & 5.60149600 & -4.14373600 & -1.14832800 \\
\hline $\mathrm{C}$ & 6.58433900 & -1.99595400 & -2.10543600 \\
\hline $\mathrm{H}$ & 5.40661500 & -4.56475900 & -2.13979400 \\
\hline $\mathrm{H}$ & 6.59539900 & -4.48031400 & -0.83910500 \\
\hline $\mathrm{H}$ & 4.86576700 & -4.54681400 & -0.44687500 \\
\hline $\mathrm{H}$ & 7.59277000 & -2.33179500 & -1.84611500 \\
\hline $\mathrm{H}$ & 6.39701700 & -2.28590200 & -3.14470000 \\
\hline $\mathrm{H}$ & 6.55940200 & -0.90234100 & -2.04550800 \\
\hline $\mathrm{C}$ & 3.49086700 & -2.53073900 & -2.66794600 \\
\hline $\mathrm{C}$ & 2.37435900 & -3.53165800 & -2.38058900 \\
\hline $\mathrm{H}$ & 1.53953600 & -3.04514600 & -1.86901300 \\
\hline $\mathrm{H}$ & 1.99507300 & -3.94154300 & -3.32153800 \\
\hline $\mathrm{H}$ & 2.72167400 & -4.35631200 & -1.75454200 \\
\hline $\mathrm{N}$ & -1.95816400 & -0.60861000 & -0.76985300 \\
\hline $\mathrm{H}$ & -1.56408800 & 0.28677500 & -1.05350900 \\
\hline $\mathrm{H}$ & 5.78338200 & -2.28339500 & -0.13847300 \\
\hline $\mathrm{H}$ & 4.28381400 & 0.94396600 & 3.40678100 \\
\hline $\mathrm{H}$ & 4.25185900 & -3.02040300 & -3.28150000 \\
\hline $\mathrm{C}$ & 2.97738800 & -1.31224200 & -3.43414700 \\
\hline $\mathrm{H}$ & 3.78151700 & -0.59186900 & -3.61868500 \\
\hline $\mathrm{H}$ & 2.56977600 & -1.62539100 & -4.39955800 \\
\hline $\mathrm{H}$ & 2.16977500 & -0.81793400 & -2.88131300 \\
\hline $\mathrm{H}$ & 6.11346000 & -0.36018300 & 0.62539400 \\
\hline $\mathrm{C}$ & 6.48129500 & 1.24069900 & 1.97465500 \\
\hline $\mathrm{H}$ & 6.13804100 & 2.03787800 & 1.30912200 \\
\hline $\mathrm{H}$ & 6.29880200 & 1.54777700 & 3.00931600 \\
\hline $\mathrm{H}$ & 7.56320800 & 1.12862300 & 1.85774900 \\
\hline $\mathrm{C}$ & -3.35207800 & -0.61973100 & -0.70466500 \\
\hline $\mathrm{C}$ & -4.08647800 & -1.65771100 & -0.12846700 \\
\hline $\mathrm{C}$ & -4.01882800 & 0.50162600 & -1.20889000 \\
\hline $\mathrm{C}$ & -5.46788700 & -1.53534900 & -0.02781500 \\
\hline $\mathrm{H}$ & -3.58754500 & -2.55341900 & 0.23545700 \\
\hline $\mathrm{C}$ & -5.39708900 & 0.60481200 & -1.08190600 \\
\hline $\mathrm{H}$ & -3.45482800 & 1.29802200 & -1.68655700 \\
\hline $\mathrm{C}$ & -6.13958500 & -0.40766500 & -0.48495400 \\
\hline $\mathrm{H}$ & -7.21476800 & -0.32584800 & -0.38853600 \\
\hline $\mathrm{C}$ & -6.05909200 & 1.88524200 & -1.51051800 \\
\hline $\mathrm{C}$ & -6.22590700 & -2.64081000 & 0.66739100 \\
\hline $\mathrm{F}$ & -7.36920100 & 1.74679000 & -1.71244000 \\
\hline $\mathrm{F}$ & -5.90494400 & 2.83795000 & -0.55003500 \\
\hline $\mathrm{F}$ & -5.51185500 & 2.38610300 & -2.62645700 \\
\hline $\mathrm{F}$ & -6.01887900 & -2.58940400 & 1.99549000 \\
\hline $\mathrm{F}$ & -7.54681800 & -2.54722000 & 0.4627300 \\
\hline
\end{tabular}




$\begin{array}{lrrr}\mathrm{F} & -5.82729600 & -3.84702300 & 0.24858000 \\ \mathrm{C} & 0.87271700 & 3.34912400 & -0.97123400 \\ \mathrm{H} & -1.64820400 & 2.47894300 & 0.30365200 \\ \mathrm{O} & -1.10502300 & 2.16072800 & -0.82670600 \\ \mathrm{~N} & 0.22740700 & 2.24213000 & -0.79636900 \\ \mathrm{C} & 2.34411000 & 3.29809100 & -1.00687800 \\ \mathrm{C} & 3.08612700 & 4.36172800 & -0.47941000 \\ \mathrm{C} & 3.01192700 & 2.20237800 & -1.56681000 \\ \mathrm{C} & 4.47462900 & 4.31215600 & -0.48170200 \\ \mathrm{H} & 2.58032600 & 5.22271600 & -0.05284400 \\ \mathrm{C} & 4.39988500 & 2.15726800 & -1.56584300 \\ \mathrm{H} & 2.44806000 & 1.39946600 & -2.03075500 \\ \mathrm{C} & 5.13299300 & 3.20916200 & -1.02274100 \\ \mathrm{H} & 5.04385500 & 5.13803500 & -0.06815900 \\ \mathrm{H} & 4.90758400 & 1.30605300 & -2.00979600 \\ \mathrm{H} & 6.21810500 & 3.18062200 & -1.03811800 \\ \mathrm{C} & 0.11762900 & 4.62842000 & -1.11927600 \\ \mathrm{H} & -0.87056300 & 4.43138600 & -1.53876900 \\ \mathrm{H} & -0.02027500 & 5.09818800 & -0.13745900 \\ \mathrm{H} & 0.66550000 & 5.32635300 & -1.75440600 \\ \mathrm{C} & -2.10627900 & 2.81941000 & 1.51322500 \\ \mathrm{C} & -1.01332800 & 2.35403800 & 2.46201600 \\ \mathrm{C} & -3.28911300 & 2.08081300 & 1.50374400 \\ \mathrm{H} & -2.25544400 & 3.88945900 & 1.36596400 \\ \mathrm{C} & -2.38347900 & 0.27055100 & 2.77548500 \\ \mathrm{H} & -1.19010400 & 2.74284100 & 3.47192200 \\ \mathrm{H} & -0.04009800 & 2.73576900 & 2.13988400 \\ \mathrm{H} & -4.17780900 & 2.41938100 & 0.97516000 \\ \mathrm{O} & -3.44881000 & 0.90286500 & 2.00438800 \\ \mathrm{C} & -1.00433000 & 0.82422600 & 2.46003900 \\ \mathrm{H} & -2.66081300 & 0.42107800 & 3.82171300 \\ \mathrm{H} & -2.47639100 & -0.78822100 & 2.53067300 \\ \mathrm{H} & -0.30217500 & 0.42543600 & 3.19827900 \\ \mathrm{H} & -0.67478700 & 0.46529000 & 1.48129200 \\ \mathrm{H} & 0.69915600 & 1.30334900 & -0.62287300\end{array}$

Structure IM2 


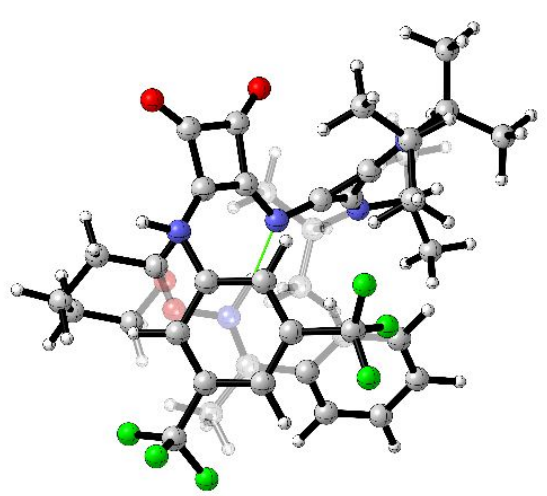

Zero-point correction=

Thermal correction to Energy=

Thermal correction to Enthalpy=

Thermal correction to Gibbs Free Energy=

Sum of electronic and zero-point Energies=

Sum of electronic and thermal Energies $=$

Sum of electronic and thermal Enthalpies=

Sum of electronic and thermal Free Energies=
0.871858 (Hartree/Particle)

0.927927

0.928871

0.777986

$-2726.127962$

$-2726.071893$

$-2726.070949$

$-2726.221834$

Number of Imaginary Frequencies $=0$

E (Single Point Energy) [IEFPCM(DCM) $\omega B$ 97X-D/6-311++G(d,p)] = -2727.778499

11

C

$\mathrm{C}$

$-0.61390500$

$-0.12916000$

3.04432000

0.48773800

0.13857100

2.24509300

$\mathrm{C}$

1.34112600

0.45998800

3.42850600

C

0.09177200

0.19319200

$-0.24011500$

0.26342100

4.30509800

$\mathrm{O}$

2.49418900

0.78468200

5.46004100

0.68291600

0.27563400

3.56052300

$\mathrm{N}$

1.96584900

0.27697700

0.91459400

C

2.94453900

0.83953600

0.49708300

3.21035000

$-0.32689100$

$-0.29662200$

C

4.04773800

$-1.29481200$

0.43235100

$\mathrm{N}$

3.36186500

0.76153300

$\mathrm{N}$

2.56928000

1.80055400

$-1.10548500$

C

1.27792900

3.04299000

$-1.22237700$

$\mathrm{C}$

C

2.30998400

2.80648500

$-1.99895800$

$\mathrm{H}$

0.60235900

3.65476500

0.15298100

$\mathrm{H}$

0.76655800

2.18134300

$-1.41247500$

$\mathrm{H}$

1.46821100

3.75645000

$-2.18011300$

$\mathrm{H}$

1.69780900

$2.31435800-2.95731700$

$\mathrm{H}$

3.24862200

2.98951300

0.77133300

1.76385200

3.84793600

0.68009900

$\mathrm{H}$

4.59589600

0.04641400 


$\begin{array}{lrrr}\mathrm{C} & 4.62057600 & 1.64106800 & -1.85654600 \\ \mathrm{C} & 5.70468300 & 2.58302300 & -1.33588500 \\ \mathrm{H} & 5.44136700 & 3.63245300 & -1.50531100 \\ \mathrm{H} & 5.86184300 & 2.44059900 & -0.26201100 \\ \mathrm{H} & 6.64970700 & 2.39262000 & -1.85341000 \\ \mathrm{C} & 5.49381700 & -1.13064700 & 0.51359800 \\ \mathrm{C} & 6.29806500 & -1.34372200 & 1.79526600 \\ \mathrm{C} & 5.96506600 & -2.01381700 & -0.64091700 \\ \mathrm{H} & 6.24451400 & -2.37967400 & 2.14526700 \\ \mathrm{H} & 7.35151800 & -1.11512300 & 1.60880100 \\ \mathrm{H} & 5.93587200 & -0.68742700 & 2.59140300 \\ \mathrm{H} & 7.01994800 & -1.82046300 & -0.85751000 \\ \mathrm{H} & 5.86793900 & -3.07755000 & -0.39920400 \\ \mathrm{H} & 5.38369600 & -1.81648000 & -1.54822400 \\ \mathrm{C} & 3.52594900 & -2.56701100 & 1.30738700 \\ \mathrm{C} & 3.03371800 & -2.43971600 & 2.74667300 \\ \mathrm{H} & 2.07165300 & -1.92060200 & 2.78288000 \\ \mathrm{H} & 2.88712200 & -3.43655300 & 3.17359500 \\ \mathrm{H} & 3.74082000 & -1.88961000 & 3.37104300 \\ \mathrm{~N} & -1.91008700 & -0.47177200 & 2.82356500 \\ \mathrm{H} & -2.52271100 & -0.43966300 & 3.62855900 \\ \mathrm{H} & 5.61745800 & -0.08225600 & 0.22604200 \\ \mathrm{H} & 3.19863900 & 3.73295300 & -1.79100300 \\ \mathrm{H} & 4.37983200 & -3.24924200 & 1.29468200 \\ \mathrm{C} & 2.44844200 & -3.13523700 & 0.38787900 \\ \mathrm{H} & 2.82612300 & -3.27490200 & -0.62999900 \\ \mathrm{H} & 2.09828100 & -4.10186300 & 0.75899400 \\ \mathrm{H} & 1.58236800 & -2.46507900 & 0.34806800 \\ \mathrm{H} & 4.93704400 & 0.61067400 & -1.66991000 \\ \mathrm{C} & 4.38438200 & 1.77713000 & -3.36077700 \\ \mathrm{H} & 3.61019200 & 1.07907800 & -3.69464100 \\ \mathrm{H} & 4.07848400 & 2.79184100 & -3.63587600 \\ \mathrm{H} & 5.30951000 & 1.55569800 & -3.90108700 \\ \mathrm{C} & -2.34333200 & -1.18965100 & 1.69279300 \\ \mathrm{C} & -1.54197200 & -2.18089100 & 1.12791100 \\ \mathrm{C} & -3.58819300 & -0.90244700 & 1.13024400 \\ \mathrm{C} & -1.98818500 & -2.87703600 & 0.00676800 \\ \mathrm{H} & -0.59534500 & -2.43619500 & 1.59250200 \\ \mathrm{H} & -4.00438300 & -1.58139700 & -0.00554900 \\ \mathrm{H} & -3.20276800 & -0.12097300 & 1.56180700 \\ \mathrm{H} & -3.20951700 & -2.57268200 & -0.57931900 \\ \mathrm{H} & -1.34989000 & -3.11270700 & -1.45460400 \\ \mathrm{H} & & -1.27486400 & -0.61321400 \\ \mathrm{H} & -3958300 & -3.99398300 & -0.55066800 \\ \mathrm{H} & -0.03725200 & -0.28787800 \\ \mathrm{H} & -1.33725400 & -1.95758200\end{array}$




$\begin{array}{lrrr}\mathrm{F} & -6.28626300 & -2.13576200 & -0.20974100 \\ \mathrm{~F} & -0.14744300 & -3.52667500 & -1.33814400 \\ \mathrm{~F} & -0.55221300 & -4.69091600 & 0.43336300 \\ \mathrm{~F} & -1.85802800 & -4.84296800 & -1.28952700 \\ \mathrm{C} & -1.89098200 & 0.86432300 & -1.63890000 \\ \mathrm{H} & -2.87601800 & 2.31544000 & 2.81791400 \\ \mathrm{O} & -2.31057100 & 1.68548800 & 0.46596300 \\ \mathrm{~N} & -1.48188600 & 1.08538700 & -0.43610600 \\ \mathrm{C} & -0.95380900 & 0.22052100 & -2.57299800 \\ \mathrm{C} & -1.00297900 & 0.57856100 & -3.92675500 \\ \mathrm{C} & 0.03599900 & -0.66226100 & -2.12748100 \\ \mathrm{C} & -0.04648100 & 0.09401900 & -4.80926400 \\ \mathrm{H} & -1.76978300 & 1.25372400 & -4.29192300 \\ \mathrm{C} & 0.98432900 & -1.14896900 & -3.01578700 \\ \mathrm{H} & 0.05192500 & -0.98635800 & -1.09383100 \\ \mathrm{C} & 0.95260900 & -0.76392600 & -4.35388200 \\ \mathrm{H} & -0.08306600 & 0.38382600 & -5.85400600 \\ \mathrm{H} & 1.73633300 & -1.84642100 & -2.66194500 \\ \mathrm{H} & 1.69369700 & -1.14769400 & -5.04801800 \\ \mathrm{C} & -3.24664700 & 1.30643900 & -2.08124500 \\ \mathrm{H} & -3.15846200 & 2.22908500 & -2.66595600 \\ \mathrm{H} & -3.69136900 & 0.54170400 & -2.72049100 \\ \mathrm{H} & -3.89979200 & 1.49364300 & -1.23157600 \\ \mathrm{C} & -2.56280400 & 3.20393700 & 2.26218900 \\ \mathrm{C} & -3.74882500 & 3.99838000 & 1.71293900 \\ \mathrm{C} & -1.61426500 & 2.77563400 & 1.15577500 \\ \mathrm{H} & -1.98142000 & 3.82978600 & 2.94817200 \\ \mathrm{C} & -2.33606300 & 4.53594700 & -0.27606600 \\ \mathrm{H} & -4.33826300 & 4.40657900 & 2.53848800 \\ \mathrm{H} & -4.41217300 & 3.33779700 & 1.14039700 \\ \mathrm{H} & -0.67894200 & 2.37358600 & 1.54917900 \\ \mathrm{O} & -1.25643800 & 3.76174300 & 0.26240700 \\ \mathrm{C} & -3.22632300 & 5.11830000 & 0.81074600 \\ \mathrm{H} & -1.85215700 & 5.31393300 & -0.86889200 \\ \mathrm{H} & -2.92990500 & 3.90567900 & -0.95210300 \\ \mathrm{H} & -2.64877000 & 5.83718700 & 1.40386400 \\ \mathrm{H} & -4.05052300 & 5.66634500 & 0.34283800 \\ \mathrm{H} & -0.53219200 & 0.75409600 & 0.01215700\end{array}$

Structure IM3 


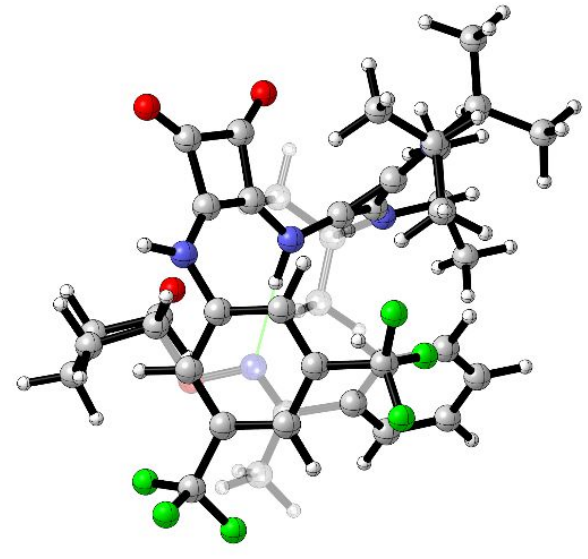

Zero-point correction=

Thermal correction to Energy=

Thermal correction to Enthalpy=

Thermal correction to Gibbs Free Energy=

Sum of electronic and zero-point Energies=

Sum of electronic and thermal Energies $=$

Sum of electronic and thermal Enthalpies=

Sum of electronic and thermal Free Energies=
0.873602 (Hartree/Particle)

0.929647

0.930591

0.780322

$-2726.146606$

$-2726.090560$

$-2726.089616$

$-2726.239885$

Number of Imaginary Frequencies $=0$

E (Single Point Energy) [IEFPCM(DCM) $\omega$ B97X-D/6-311++G(d,p)] = -2727.793929

11

C

$\mathrm{C}$

$\mathrm{C}$

C

$\mathrm{O}$

$\mathrm{O}$

$\mathrm{N}$

C

C

C

$\mathrm{N}$

$\mathrm{N}$

C

C

C

$\mathrm{H}$

$\mathrm{H}$

$\mathrm{H}$

$\begin{array}{rrr}-0.65730800 & -0.65784600 & 3.12399700 \\ 0.37805500 & -0.16958500 & 2.35674300 \\ 1.28410900 & 0.02243400 & 3.51029400 \\ 0.11150600 & -0.47666700 & 4.40042500 \\ -0.15299300 & -0.62822900 & 5.55835700 \\ 2.42804700 & 0.37703900 & 3.63145900 \\ 0.56529300 & 0.11541900 & 1.02604400 \\ 1.83760500 & 0.20666700 & 0.55024400 \\ 2.81689900 & 0.90160300 & -0.11615500 \\ 3.06605900 & -0.39108000 & 0.39415800 \\ 3.89941200 & -1.39270600 & 0.57157100 \\ 3.24546600 & 1.97469700 & -0.74068400 \\ 2.50975900 & 3.25302100 & -0.58731500 \\ 1.21541600 & 3.25127600 & -1.39195600 \\ 2.27638900 & 3.57228700 & 0.88844100 \\ 0.48864400 & 2.56084500 & -0.95366900 \\ 0.77047200 & 4.25120200 & -1.37735100 \\ 1.39137000 & 2.96247500 & -2.43138400\end{array}$




\begin{tabular}{|c|c|c|c|}
\hline $\mathrm{H}$ & 1.51677100 & 2.91621000 & 1.32716000 \\
\hline $\mathrm{H}$ & 3.20068400 & 3.48105800 & 1.46628000 \\
\hline $\mathrm{H}$ & 1.91505600 & 4.60042500 & 0.98469900 \\
\hline $\mathrm{C}$ & 4.49936000 & 1.92351200 & -1.52224800 \\
\hline $\mathrm{C}$ & 5.62470500 & 2.67585500 & -0.81497400 \\
\hline $\mathrm{H}$ & 5.41448800 & 3.74857700 & -0.75310600 \\
\hline $\mathrm{H}$ & 5.77173300 & 2.29993200 & 0.20282500 \\
\hline $\mathrm{H}$ & 6.56091900 & 2.55496900 & -1.36792500 \\
\hline $\mathrm{C}$ & 5.34652200 & -1.19402700 & 0.33486300 \\
\hline $\mathrm{C}$ & 6.16065000 & -1.56178400 & 1.57392700 \\
\hline $\mathrm{C}$ & 5.80222300 & -1.92581300 & -0.92567100 \\
\hline $\mathrm{H}$ & 6.11754100 & -2.63354100 & 1.79214300 \\
\hline $\mathrm{H}$ & 7.21066600 & -1.30435700 & 1.40768600 \\
\hline $\mathrm{H}$ & 5.80407400 & -1.01165600 & 2.44924800 \\
\hline $\mathrm{H}$ & 6.85440500 & -1.70475000 & -1.12743500 \\
\hline $\mathrm{H}$ & 5.70892400 & -3.01160100 & -0.81833100 \\
\hline $\mathrm{H}$ & 5.21046700 & -1.61619000 & -1.79386200 \\
\hline $\mathrm{C}$ & 3.39020200 & -2.72755100 & 0.96622600 \\
\hline $\mathrm{C}$ & 2.91088100 & -2.76948100 & 2.41463500 \\
\hline $\mathrm{H}$ & 1.94022200 & -2.27523200 & 2.51515100 \\
\hline $\mathrm{H}$ & 2.78363800 & -3.81019700 & 2.72712600 \\
\hline $\mathrm{H}$ & 3.61584500 & -2.28327400 & 3.09238400 \\
\hline $\mathrm{N}$ & -1.90393300 & -1.10588300 & 2.90162700 \\
\hline $\mathrm{H}$ & -2.48087400 & -1.28775800 & 3.71328600 \\
\hline $\mathrm{H}$ & 5.46499200 & -0.11848200 & 0.17818700 \\
\hline $\mathrm{H}$ & 3.18149600 & 4.01043100 & -0.99931800 \\
\hline $\mathrm{H}$ & 4.25113600 & -3.39319500 & 0.86983600 \\
\hline $\mathrm{C}$ & 2.31330700 & -3.19577800 & -0.00666500 \\
\hline $\mathrm{H}$ & 2.69768500 & -3.25015600 & -1.02944400 \\
\hline $\mathrm{H}$ & 1.95262800 & -4.18880400 & 0.27350500 \\
\hline $\mathrm{H}$ & 1.45321300 & -2.51670100 & 0.00450400 \\
\hline $\mathrm{H}$ & 4.76122300 & 0.86230700 & -1.57263300 \\
\hline $\mathrm{C}$ & 4.26799300 & 2.40030200 & -2.95506200 \\
\hline $\mathrm{H}$ & 3.45825600 & 1.83370000 & -3.42439000 \\
\hline $\mathrm{H}$ & 4.01933200 & 3.46557900 & -2.99682700 \\
\hline $\mathrm{H}$ & 5.18143000 & 2.25412400 & -3.53901500 \\
\hline $\mathrm{C}$ & -2.34372800 & -1.55551700 & 1.62692300 \\
\hline $\mathrm{C}$ & -1.59327400 & -2.49893700 & 0.92896000 \\
\hline $\mathrm{C}$ & -3.51874400 & -1.05065300 & 1.08072800 \\
\hline $\mathrm{C}$ & -2.02509100 & -2.93247100 & -0.32013400 \\
\hline $\mathrm{H}$ & -0.69896300 & -2.91645000 & 1.38006200 \\
\hline $\mathrm{C}$ & -3.92439900 & -1.47165000 & -0.17919500 \\
\hline $\mathrm{H}$ & -4.09634200 & -0.31464400 & 1.62820800 \\
\hline $\mathrm{C}$ & -3.18270400 & -2.41099300 & -0.88757600 \\
\hline $\mathrm{H}$ & -3.51249800 & -2.74495900 & -1.86445800 \\
\hline $\mathrm{C}$ & -5.20411000 & -0.93344100 & -0.76985000 \\
\hline
\end{tabular}




$\begin{array}{lrrr}\mathrm{C} & -1.23228200 & -3.98513200 & -1.05612600 \\ \mathrm{~F} & -5.48174700 & 0.29566700 & -0.30270600 \\ \mathrm{~F} & -5.12015000 & -0.84778100 & -2.10707200 \\ \mathrm{~F} & -6.24562300 & -1.71972300 & -0.47894900 \\ \mathrm{~F} & -0.24224600 & -3.44118200 & -1.79234800 \\ \mathrm{~F} & -0.65131300 & -4.84039700 & -0.19960300 \\ \mathrm{~F} & -2.00122700 & -4.68932500 & -1.88915200 \\ \mathrm{C} & -1.80984500 & 1.18982300 & -1.91017100 \\ \mathrm{H} & -4.44078900 & 2.08177000 & 1.81957500 \\ \mathrm{O} & -2.71127000 & 1.80331100 & 0.01371100 \\ \mathrm{~N} & -1.64696900 & 1.20110500 & -0.63580100 \\ \mathrm{C} & -0.68866800 & 0.63350300 & -2.70412500 \\ \mathrm{C} & -0.34222000 & 1.23100400 & -3.92103700 \\ \mathrm{C} & 0.08506600 & -0.42610700 & -2.22441300 \\ \mathrm{C} & 0.77796300 & 0.80075900 & -4.62388100 \\ \mathrm{H} & -0.93320700 & 2.05383400 & -4.31190800 \\ \mathrm{C} & 1.20342800 & -0.85656300 & -2.92846200 \\ \mathrm{H} & -0.20916900 & -0.92903200 & -1.31020200 \\ \mathrm{C} & 1.55824300 & -0.24027600 & -4.12562800 \\ \mathrm{H} & 1.03854900 & 1.27607900 & -5.56428400 \\ \mathrm{H} & 1.78200900 & -1.69440700 & -2.55296100 \\ \mathrm{H} & 2.42450000 & -0.58518300 & -4.68217900 \\ \mathrm{C} & -3.01035200 & 1.79334000 & -2.58051700 \\ \mathrm{H} & -2.95201100 & 2.88751500 & -2.54267300 \\ \mathrm{H} & -3.08239100 & 1.47714600 & -3.62178500 \\ \mathrm{H} & -3.92257200 & 1.50550000 & -2.05692400 \\ \mathrm{C} & -3.59329400 & 2.75700800 & 1.97297300 \\ \mathrm{C} & -3.87917400 & 4.14369200 & 1.39074000 \\ \mathrm{C} & -2.34679300 & 2.14487000 & 1.34800800 \\ \mathrm{H} & -3.41242900 & 2.83300300 & 3.05101900 \\ \mathrm{C} & -1.45309400 & 4.27083200 & 0.79953000 \\ \mathrm{H} & -4.69983500 & 4.61697100 & 1.93746800 \\ \mathrm{H} & -4.20056100 & 4.04411400 & 0.34726800 \\ \mathrm{H} & -2.03440000 & 1.23907200 & 1.87487800 \\ \mathrm{O} & -1.23412000 & 2.98215400 & 1.37807700 \\ \mathrm{C} & -2.61257500 & 4.99925400 & 1.46351800 \\ \mathrm{H} & -0.51030800 & 4.80485800 & 0.93393400 \\ \mathrm{H} & -1.63464100 & 4.15803700 & -0.27880600 \\ \mathrm{H} & -2.35566900 & 5.19882200 & 2.51085300 \\ \mathrm{H} & -2.76252100 & 5.96643000 & 0.97211500 \\ \mathrm{H} & & 0.56948800 & 0.48277900\end{array}$

9. Parameters for OPLS4 Conformer Searches 
The following parameters were used for the conformational searches:

Potential (parameters)

Force field $=$ OPLS4

Solvent $=$ none

Electrostatic treatment $=$ constant dielectric

Dielectric constant $=1$

Charges from force field

Cutoff $=$ normal

Van der Waals $=7.0$

Electrostatic $=12.0$

H-bond $=4$

Mini (parameters)

Method $=$ PRCG

Maximum interactions $=2500$

Converge on $=$ Gradient

Convergence threshold $=0.05$

CSearch (parameters)

Method $=$ Mixed torsional/Low-mode sampling

Torsion sampling option $=$ intermediate

Maximum number of steps $=1000$ (using 100 steps per rotatable bond)

Number of structures saved from search $=100$

Energy window for saving structures $=5.02 \mathrm{kcal} / \mathrm{mol}$

Eliminate redundant conformers using: Maximum atom deviation Cutoff $=0.5$ Angstrom

Probability of a torsion rotation $/$ molecule transition $=0.5$

Minimum distance for low-mode move $=3.0$

Maximum distance for low-mode move $=6.0$

\section{TS1 IRC Coordinates}




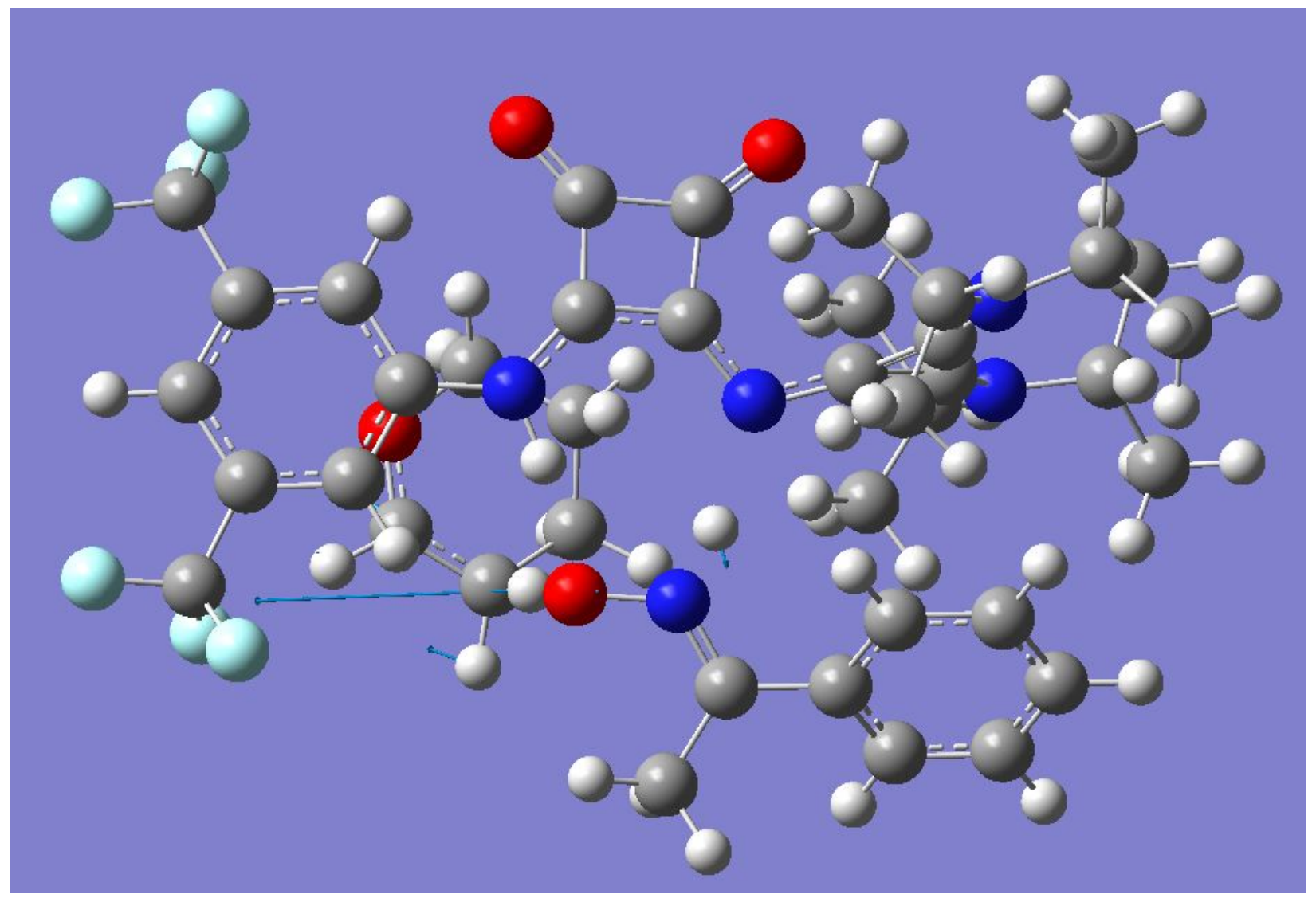

Figure S6. Analysis of TS1 with displacement vectors depicted.

\section{TS1 IRC Coordinates Step 1}

$\begin{array}{lrrr}11 & & & \\ \mathrm{C} & -1.06638300 & -1.49728000 & -0.26163500 \\ \mathrm{C} & 0.31305100 & -1.34981100 & -0.11492400 \\ \mathrm{C} & 0.45192700 & -2.68975600 & 0.49941400 \\ \mathrm{C} & -1.06509900 & -2.85516800 & 0.34128900 \\ \mathrm{O} & -1.89031700 & -3.68441000 & 0.64921600 \\ \mathrm{O} & 1.38664600 & -3.31212000 & 0.95120500 \\ \mathrm{~N} & 1.16345200 & -0.32242000 & -0.27714800 \\ \mathrm{C} & 2.47199700 & -0.64642400 & -0.13315300 \\ \mathrm{C} & 3.66273700 & -0.45924800 & 0.53000800 \\ \mathrm{C} & 3.60068600 & -1.30776600 & -0.58464900 \\ \mathrm{~N} & 4.20176300 & -2.11405500 & -1.43834200 \\ \mathrm{~N} & 4.33197300 & 0.08497500 & 1.53501600 \\ \mathrm{C} & 3.54796000 & 0.62853200 & 2.66324600 \\ \mathrm{C} & 2.75210500 & 1.85479100 & 2.22301100 \\ \mathrm{C} & 2.66026100 & -0.44805300 & 3.28860500 \\ \mathrm{H} & 1.98111800 & 1.56802200 & 1.49835800 \\ \mathrm{H} & 2.25587900 & 2.30838800 & 3.08745600\end{array}$




\begin{tabular}{|c|c|c|c|}
\hline $\mathrm{H}$ & 3.40569200 & 2.60051700 & 1.76156900 \\
\hline $\mathrm{H}$ & 1.90916100 & -0.80689500 & 2.57662700 \\
\hline $\mathrm{H}$ & 3.25095200 & -1.30926500 & 3.61254800 \\
\hline $\mathrm{H}$ & 2.14044900 & -0.04145800 & 4.16231200 \\
\hline $\mathrm{C}$ & 5.79315000 & -0.08073100 & 1.63436600 \\
\hline $\mathrm{C}$ & 6.16454500 & -1.20150400 & 2.60419800 \\
\hline $\mathrm{H}$ & 5.86063000 & -0.95210700 & 3.62671300 \\
\hline $\mathrm{H}$ & 5.67764900 & -2.14168600 & 2.32492500 \\
\hline $\mathrm{H}$ & 7.24746600 & -1.35855300 & 2.60891600 \\
\hline $\mathrm{C}$ & 5.56100700 & -2.61643100 & -1.15578600 \\
\hline $\mathrm{C}$ & 5.60149600 & -4.14373600 & -1.14832800 \\
\hline $\mathrm{C}$ & 6.58433900 & -1.99595400 & -2.10543600 \\
\hline $\mathrm{H}$ & 5.40661500 & -4.56475900 & -2.13979400 \\
\hline $\mathrm{H}$ & 6.59539900 & -4.48031400 & -0.83910500 \\
\hline $\mathrm{H}$ & 4.86576700 & -4.54681400 & -0.44687500 \\
\hline $\mathrm{H}$ & 7.59277000 & -2.33179500 & -1.84611500 \\
\hline $\mathrm{H}$ & 6.39701700 & -2.28590200 & -3.14470000 \\
\hline $\mathrm{H}$ & 6.55940200 & -0.90234100 & -2.04550800 \\
\hline $\mathrm{C}$ & 3.49086700 & -2.53073900 & -2.66794600 \\
\hline $\mathrm{C}$ & 2.37435900 & -3.53165800 & -2.38058900 \\
\hline $\mathrm{H}$ & 1.53953600 & -3.04514600 & -1.86901300 \\
\hline $\mathrm{H}$ & 1.99507300 & -3.94154300 & -3.32153800 \\
\hline $\mathrm{H}$ & 2.72167400 & -4.35631200 & -1.75454200 \\
\hline $\mathrm{N}$ & -1.95816400 & -0.60861000 & -0.76985300 \\
\hline $\mathrm{H}$ & -1.56408800 & 0.28677500 & -1.05350900 \\
\hline $\mathrm{H}$ & 5.78338200 & -2.28339500 & -0.13847300 \\
\hline $\mathrm{H}$ & 4.28381400 & 0.94396600 & 3.40678100 \\
\hline $\mathrm{H}$ & 4.25185900 & -3.02040300 & -3.28150000 \\
\hline $\mathrm{C}$ & 2.97738800 & -1.31224200 & -3.43414700 \\
\hline $\mathrm{H}$ & 3.78151700 & -0.59186900 & -3.61868500 \\
\hline $\mathrm{H}$ & 2.56977600 & -1.62539100 & -4.39955800 \\
\hline $\mathrm{H}$ & 2.16977500 & -0.81793400 & -2.88131300 \\
\hline $\mathrm{H}$ & 6.11346000 & -0.36018300 & 0.62539400 \\
\hline $\mathrm{C}$ & 6.48129500 & 1.24069900 & 1.97465500 \\
\hline $\mathrm{H}$ & 6.13804100 & 2.03787800 & 1.30912200 \\
\hline $\mathrm{H}$ & 6.29880200 & 1.54777700 & 3.00931600 \\
\hline $\mathrm{H}$ & 7.56320800 & 1.12862300 & 1.85774900 \\
\hline $\mathrm{C}$ & -3.35207800 & -0.61973100 & -0.70466500 \\
\hline $\mathrm{C}$ & -4.08647800 & -1.65771100 & -0.12846700 \\
\hline $\mathrm{C}$ & -4.01882800 & 0.50162600 & -1.20889000 \\
\hline $\mathrm{C}$ & -5.46788700 & -1.53534900 & -0.02781500 \\
\hline $\mathrm{H}$ & -3.58754500 & -2.55341900 & 0.23545700 \\
\hline $\mathrm{C}$ & -5.39708900 & 0.60481200 & -1.08190600 \\
\hline $\mathrm{H}$ & -3.45482800 & 1.29802200 & -1.6865570 \\
\hline $\mathrm{C}$ & -6.13958500 & -0.40766500 & -0.48495400 \\
\hline $\mathrm{H}$ & -7.21476800 & -0.32584800 & -0.3885360 \\
\hline
\end{tabular}




\begin{tabular}{|c|c|c|c|}
\hline $\mathrm{C}$ & -6.05909200 & 1.88524200 & -1.51051800 \\
\hline $\mathrm{C}$ & -6.22590700 & -2.64081000 & 0.66739100 \\
\hline $\mathrm{F}$ & -7.36920100 & 1.74679000 & -1.71244000 \\
\hline $\mathrm{F}$ & -5.90494400 & 2.83795000 & -0.55003500 \\
\hline $\mathrm{F}$ & -5.51185500 & 2.38610300 & -2.62645700 \\
\hline $\mathrm{F}$ & -6.01887900 & -2.58940400 & 1.99549000 \\
\hline $\mathrm{F}$ & -7.54681800 & -2.54722000 & 0.46273000 \\
\hline $\mathrm{F}$ & -5.82729600 & -3.84702300 & 0.24858000 \\
\hline $\mathrm{C}$ & 0.87271700 & 3.34912400 & -0.97123400 \\
\hline $\mathrm{H}$ & -1.64820400 & 2.47894300 & 0.30365200 \\
\hline $\mathrm{O}$ & -1.10502300 & 2.16072800 & -0.82670600 \\
\hline $\mathrm{N}$ & 0.22740700 & 2.24213000 & -0.79636900 \\
\hline $\mathrm{C}$ & 2.34411000 & 3.29809100 & -1.00687800 \\
\hline $\mathrm{C}$ & 3.08612700 & 4.36172800 & -0.47941000 \\
\hline $\mathrm{C}$ & 3.01192700 & 2.20237800 & -1.56681000 \\
\hline $\mathrm{C}$ & 4.47462900 & 4.31215600 & -0.48170200 \\
\hline $\mathrm{H}$ & 2.58032600 & 5.22271600 & -0.05284400 \\
\hline $\mathrm{C}$ & 4.39988500 & 2.15726800 & -1.56584300 \\
\hline $\mathrm{H}$ & 2.44806000 & 1.39946600 & -2.03075500 \\
\hline $\mathrm{C}$ & 5.13299300 & 3.20916200 & -1.02274100 \\
\hline $\mathrm{H}$ & 5.04385500 & 5.13803500 & -0.06815900 \\
\hline $\mathrm{H}$ & 4.90758400 & 1.30605300 & -2.00979600 \\
\hline $\mathrm{H}$ & 6.21810500 & 3.18062200 & -1.03811800 \\
\hline $\mathrm{C}$ & 0.11762900 & 4.62842000 & -1.11927600 \\
\hline $\mathrm{H}$ & -0.87056300 & 4.43138600 & -1.53876900 \\
\hline $\mathrm{H}$ & -0.02027500 & 5.09818800 & -0.13745900 \\
\hline $\mathrm{H}$ & 0.66550000 & 5.32635300 & -1.75440600 \\
\hline $\mathrm{C}$ & -2.10627900 & 2.81941000 & 1.51322500 \\
\hline $\mathrm{C}$ & -1.01332800 & 2.35403800 & 2.46201600 \\
\hline $\mathrm{C}$ & -3.28911300 & 2.08081300 & 1.50374400 \\
\hline $\mathrm{H}$ & -2.25544400 & 3.88945900 & 1.36596400 \\
\hline $\mathrm{C}$ & -2.38347900 & 0.27055100 & 2.77548500 \\
\hline $\mathrm{H}$ & -1.19010400 & 2.74284100 & 3.47192200 \\
\hline $\mathrm{H}$ & -0.04009800 & 2.73576900 & 2.13988400 \\
\hline $\mathrm{H}$ & -4.17780900 & 2.41938100 & 0.97516000 \\
\hline $\mathrm{O}$ & -3.44881000 & 0.90286500 & 2.00438800 \\
\hline $\mathrm{C}$ & -1.00433000 & 0.82422600 & 2.46003900 \\
\hline $\mathrm{H}$ & -2.66081300 & 0.42107800 & 3.82171300 \\
\hline $\mathrm{H}$ & -2.47639100 & -0.78822100 & 2.53067300 \\
\hline $\mathrm{H}$ & -0.30217500 & 0.42543600 & 3.19827900 \\
\hline $\mathrm{H}$ & -0.67478700 & 0.46529000 & 1.48129200 \\
\hline $\mathrm{H}$ & 0.69915600 & 1.30334900 & -0.62287300 \\
\hline
\end{tabular}

TS1 IRC Coordinates Step 40 


\begin{tabular}{|c|c|c|c|}
\hline & & & \\
\hline $\mathrm{C}$ & -0.87562800 & -1.47720000 & -0.28186500 \\
\hline $\mathrm{C}$ & 0.49598600 & -1.30065800 & -0.11795200 \\
\hline $\mathrm{C}$ & 0.66088800 & -2.62411800 & 0.51873200 \\
\hline $\mathrm{C}$ & -0.85165300 & -2.82760000 & 0.34918100 \\
\hline $\mathrm{O}$ & -1.66086100 & -3.66921500 & 0.65490600 \\
\hline $\mathrm{O}$ & 1.60770700 & -3.22385200 & 0.97652100 \\
\hline $\mathrm{N}$ & 1.34303700 & -0.26234200 & -0.29500700 \\
\hline $\mathrm{C}$ & 2.65496300 & -0.58557900 & -0.13761000 \\
\hline $\mathrm{C}$ & 3.84007000 & -0.41931100 & 0.54100600 \\
\hline $\mathrm{C}$ & 3.77543500 & -1.26794400 & -0.57647500 \\
\hline $\mathrm{N}$ & 4.37354400 & -2.08707200 & -1.41775300 \\
\hline $\mathrm{N}$ & 4.51481300 & 0.10861100 & 1.54928600 \\
\hline $\mathrm{C}$ & 3.73981700 & 0.64422000 & 2.68920100 \\
\hline $\mathrm{C}$ & 2.94939500 & 1.87905700 & 2.26697900 \\
\hline $\mathrm{C}$ & 2.85113500 & -0.43531100 & 3.30664100 \\
\hline $\mathrm{H}$ & 2.17316700 & 1.60436400 & 1.54368600 \\
\hline $\mathrm{H}$ & 2.45609800 & 2.32358800 & 3.13713000 \\
\hline $\mathrm{H}$ & 3.60612600 & 2.62712200 & 1.81351500 \\
\hline $\mathrm{H}$ & 2.09439700 & -0.78284700 & 2.59542800 \\
\hline $\mathrm{H}$ & 3.43998500 & -1.30243100 & 3.61776500 \\
\hline $\mathrm{H}$ & 2.33668500 & -0.03626700 & 4.18656100 \\
\hline $\mathrm{C}$ & 5.97554400 & -0.06972500 & 1.64492600 \\
\hline $\mathrm{C}$ & 6.34083300 & -1.19314300 & 2.61373600 \\
\hline $\mathrm{H}$ & 6.04388600 & -0.94106500 & 3.63750100 \\
\hline $\mathrm{H}$ & 5.84471900 & -2.12931500 & 2.33738600 \\
\hline $\mathrm{H}$ & 7.42237900 & -1.35910700 & 2.61346900 \\
\hline $\mathrm{C}$ & 5.72877900 & -2.59705200 & -1.12408900 \\
\hline $\mathrm{C}$ & 5.76394800 & -4.12425500 & -1.12040900 \\
\hline $\mathrm{C}$ & 6.76179100 & -1.97722500 & -2.06365700 \\
\hline $\mathrm{H}$ & 5.57742600 & -4.54311700 & -2.11431700 \\
\hline $\mathrm{H}$ & 6.75417700 & -4.46361800 & -0.80277400 \\
\hline $\mathrm{H}$ & 5.02146300 & -4.52684800 & -0.42591200 \\
\hline $\mathrm{H}$ & 7.76669700 & -2.31860800 & -1.79820200 \\
\hline $\mathrm{H}$ & 6.58094000 & -2.26208900 & -3.10546100 \\
\hline $\mathrm{H}$ & 6.74179600 & -0.88363800 & -1.99933300 \\
\hline $\mathrm{C}$ & 3.66940400 & -2.50720700 & -2.65071700 \\
\hline $\mathrm{C}$ & 2.55088600 & -3.50655100 & -2.36612900 \\
\hline $\mathrm{H}$ & 1.71266400 & -3.01755200 & -1.86230600 \\
\hline $\mathrm{H}$ & 2.17806100 & -3.92071400 & -3.30773700 \\
\hline $\mathrm{H}$ & 2.89382300 & -4.32847700 & -1.73426800 \\
\hline $\mathrm{N}$ & -1.78105100 & -0.63983800 & -0.84133000 \\
\hline $\mathrm{H}$ & -1.39637900 & 0.22450500 & -1.20339900 \\
\hline $\mathrm{H}$ & 5.94308300 & -2.26846300 & -0.10390000 \\
\hline $\mathrm{H}$ & 4.48247400 & 0.94738900 & 3.43100600 \\
\hline $\mathrm{H}$ & 4.43393300 & -2.99896300 & -3.25802100 \\
\hline
\end{tabular}




\begin{tabular}{|c|c|c|c|}
\hline $\mathrm{C}$ & 3.16170400 & -1.29122100 & -3.42440900 \\
\hline $\mathrm{H}$ & 3.96728900 & -0.57146400 & -3.60557600 \\
\hline $\mathrm{H}$ & 2.76200300 & -1.60781400 & -4.39193000 \\
\hline $\mathrm{H}$ & 2.34907800 & -0.79577900 & -2.87985100 \\
\hline $\mathrm{H}$ & 6.29166800 & -0.35082600 & 0.63517600 \\
\hline $\mathrm{C}$ & 6.67379800 & 1.24671700 & 1.98439800 \\
\hline $\mathrm{H}$ & 6.33763600 & 2.04618200 & 1.31788600 \\
\hline $\mathrm{H}$ & 6.49255600 & 1.55667800 & 3.01831000 \\
\hline $\mathrm{H}$ & 7.75487000 & 1.12599800 & 1.86860800 \\
\hline $\mathrm{C}$ & -3.17736700 & -0.63575800 & -0.73544200 \\
\hline $\mathrm{C}$ & -3.90376800 & -1.66364100 & -0.13734500 \\
\hline $\mathrm{C}$ & -3.84255800 & 0.48640300 & -1.23272100 \\
\hline $\mathrm{C}$ & -5.28189300 & -1.52890900 & -0.00951100 \\
\hline $\mathrm{H}$ & -3.40455800 & -2.55879900 & 0.22494900 \\
\hline $\mathrm{C}$ & -5.21546100 & 0.60549800 & -1.07743300 \\
\hline $\mathrm{H}$ & -3.28503800 & 1.27774500 & -1.72558100 \\
\hline $\mathrm{C}$ & -5.95241000 & -0.39799400 & -0.45948500 \\
\hline $\mathrm{H}$ & -7.02471600 & -0.30660900 & -0.34160100 \\
\hline $\mathrm{C}$ & -5.87523100 & 1.88803000 & -1.50446400 \\
\hline $\mathrm{C}$ & -6.03813800 & -2.62805300 & 0.69669200 \\
\hline $\mathrm{F}$ & -7.18440400 & 1.74582700 & -1.71716200 \\
\hline $\mathrm{F}$ & -5.72842600 & 2.83994700 & -0.54971100 \\
\hline $\mathrm{F}$ & -5.32179800 & 2.38608900 & -2.62202900 \\
\hline $\mathrm{F}$ & -5.82670000 & -2.57436200 & 2.02250800 \\
\hline $\mathrm{F}$ & -7.35993200 & -2.53367300 & 0.49551700 \\
\hline $\mathrm{F}$ & -5.64363100 & -3.83763000 & 0.27915600 \\
\hline $\mathrm{C}$ & 1.08917200 & 3.33094000 & -0.93793100 \\
\hline $\mathrm{H}$ & -1.31340900 & 2.35308700 & 0.01046600 \\
\hline $\mathrm{O}$ & -0.90224200 & 2.17749200 & -0.89121200 \\
\hline $\mathrm{N}$ & 0.46147200 & 2.21603700 & -0.75805100 \\
\hline $\mathrm{C}$ & 2.55755900 & 3.29473300 & -0.96904800 \\
\hline $\mathrm{C}$ & 3.28863600 & 4.36551400 & -0.43842100 \\
\hline $\mathrm{C}$ & 3.22989100 & 2.20918400 & -1.54338300 \\
\hline $\mathrm{C}$ & 4.67643500 & 4.32983300 & -0.45081200 \\
\hline $\mathrm{H}$ & 2.77652500 & 5.21597900 & 0.00118600 \\
\hline $\mathrm{C}$ & 4.61782900 & 2.18316400 & -1.55849300 \\
\hline $\mathrm{H}$ & 2.66914500 & 1.40548400 & -2.00921800 \\
\hline $\mathrm{C}$ & 5.34122500 & 3.23926200 & -1.01057800 \\
\hline $\mathrm{H}$ & 5.24070800 & 5.15651000 & -0.03264500 \\
\hline $\mathrm{H}$ & 5.13267000 & 1.34385600 & -2.01599400 \\
\hline $\mathrm{H}$ & 6.42637900 & 3.22387000 & -1.03543700 \\
\hline $\mathrm{C}$ & 0.33328500 & 4.60939400 & -1.09230100 \\
\hline $\mathrm{H}$ & -0.62486500 & 4.43088500 & -1.58247200 \\
\hline $\mathrm{H}$ & 0.12629000 & 5.02766000 & -0.09923100 \\
\hline $\mathrm{H}$ & 0.91683100 & 5.33577900 & -1.65815600 \\
\hline $\mathrm{C}$ & -1.91364400 & 2.91651900 & 1.76657700 \\
\hline
\end{tabular}




$\begin{array}{lrrr}\mathrm{C} & -0.75068700 & 2.39393200 & 2.57215600 \\ \mathrm{C} & -2.99845300 & 2.14684700 & 1.53315200 \\ \mathrm{H} & -1.96617100 & 3.96458700 & 1.48451300 \\ \mathrm{C} & -2.17578000 & 0.34823100 & 2.82473100 \\ \mathrm{H} & -0.80920800 & 2.75211600 & 3.60823700 \\ \mathrm{H} & 0.19938100 & 2.77016400 & 2.17361500 \\ \mathrm{H} & -3.85560800 & 2.49056900 & 0.96211100 \\ \mathrm{O} & -3.15613400 & 0.88210000 & 1.92377900 \\ \mathrm{C} & -0.77243900 & 0.86276400 & 2.53227700 \\ \mathrm{H} & -2.48208700 & 0.61450300 & 3.84301600 \\ \mathrm{H} & -2.24607800 & -0.73478300 & 2.70909000 \\ \mathrm{H} & -0.07736600 & 0.43277900 & 3.25965000 \\ \mathrm{H} & -0.45603600 & 0.51093000 & 1.54414200 \\ \mathrm{H} & 0.90268700 & 1.21161400 & -0.56375800\end{array}$

11. References 
1. Ohwada, T.; Itai, A.; Ohta, T.; Shudo, K. Nitroethylene Yields (N,Ndihydroxyiminium)methylium Dication in Trifluoromethanesulfonic Acid. Dications Stabilized by Y Delocalization. J. Am. Chem. Soc. 1987, 109, 7036-7041.

2. Bridges, A. J.; Zhou, H. Synthesis of [1]Benzothieno[3,2-d]pyrimidines Substituted with Electron Donating Substituents on the Benzene Ring. J. Heterocyclic. Chem. 1997, 34, 1163-1172.

3. Gamble, D. L.; Hems, W. P.; Ridge B. Studies on the Preparation of Camphorylidene Derivatives of $\alpha$-Amino Acids. J. Chem. Soc. 2001, 3, 248-260.

4. Kithara, K.; Toma, T.; Shimokawa, J.; Fukuyama, T. $O$-TBS- $N$-tosylhydroxylamine: A Reagent for Facile Conversion of Alcohols to Oximes. Org. Lett. 2008, 10, 2259-2261.

5. Augustine, J. K.; Kumar, R.; Bombrun, A.; Mandal, A. B. An Efficient Catalytic Method for the Beckmann Rearrangement of Ketoximes to Amides and Aldoximes to Nitriles Mediated by Propylphosphonic Anhydride (T3P $\left.{ }^{\circledR}\right)$. Tetrahedron Lett. 2011, 52, 10741077.

6. Zaware, P.; Shah, S.; Pingali, H.; Makadia, P.; Thube, B.; Pola, S.; Patel, D.; Priyadarshini, P.; Suthar, D.; Shah, M.; Jamili, J.; Sairam, K.; Giri, S.; Patel, L.; Patel, H.; Sudani, H.; Patel, H.; Jain, M.; Patel, P.; Bahekar, R. Modulation of PPAR Subtype Selectivity. Part 2: Transforming PPAR $\alpha / \gamma$ Dual Agonist into $\alpha$ Selective PPAR Agonist through Bioisosteric Modification. Bioorg. Med. Chem. Lett. 2011, 21, 628-632.

7. Abedi, S.; Karimi, B.; Kazemi, F.; Bostina, M.; Vali, H. Amorphous $\mathrm{TiO}_{2}$ coated into Periodic Mesoporous Organosilicate Channels as a New Binary Photocatalyst for Regeneration of Carbonyl Compounds from Oximes under the Sunlight Irradiation. Org. Biomol. Chem. 2012, 11, 416-419.

8. Suzuki, K.; Watanabe, T.; Murahashi, S. Oxidation of Primary Amines to Oximes with Molecular Oxygen using 1,1-Diphenyl-2-picryhydrazyl and $\mathrm{WO}_{3} / \mathrm{Al}_{2} \mathrm{O}_{3}$ as Catalysts. $J$. Org. Chem. 2013, 78, 2301-2310.

9. Chalyk, B.; Kandaurova, I.; Hrebeniuk, K.; Manoilenko, O.; Kulik, I.; Iminov, R.; Kubyshkin, V.; Tverdekohlebov, A.; Ablialimov O.; Mykhailiuk P. A Base Promoted Multigram Synthesis of Aminosoxazoles; Valuable Building Block for Drug Discovery and Peptidomimetics. RSC. Adv. 2016, 6, 25713-25723.

10. Betke, T.; Rommelmann, P.; Oike, K.; Asano, Y.; Gröger, H. Cyanide-Free and Broadly Applicable Enantioselective Synthetic Platform for Chiral Nitriles through a Biocatalytic Approach. Angew. Chem. Int. Ed. 2017, 56, 2361-2366.

11. Kadam, K.; Gandhi, T.; Gupte, A.; Gangopadhyay, A. K.; Sharma, R. Alkyl Nitriles: Novel Reagents for One-Pot Synthesis of 3,5-Disubstitued Isoxazoles from Aldoximes and Alkynes. Synthesis 2016, 48, 3996-4008.

12. Blangetti, M.; Rolando, B.; Marini, E.; Chegev, K.; Guglielmo, S.; Lazzarato, L.; Lucarini, L.; Masini, E.; Fruttero, R. gem-Dinitroalkyl Benzenes: A Novel Class of IOP-Lowering Agents for the Treatment of Ocular Hypertension. ACS. Med. Chem. Lett. 2017, 8, 10541059.

13. Zhao, G.; Liang, L.; Wen, C.; Tong, R. In Situ Generation of Nitrile from NaCl-Oxone Oxidation of Various Aldoximes and Their 1,3-Dipolar Cycloaddition. Org. Lett. 2019, 21 , 315-319.

14. Colin, B.; Lavastre, O.; Fouquay, S.; Michaud, G.; Simon, F.; Brusson, J.; HighThroughput Screening of the Alkoxide/Oxime-Based Library: An Alternative to Organotin 
Compounds or the Alkoxysilane Condensation in Adhesive and Sealants. ACS. Comb. Sci. 2019, 21, 300-309.

15. Smajlagic, I.; Durán, R.; Pilkington, M.; Dudding. T. Cyclopropenium Enhanced Thiourea Catalysis. J. Org. Chem. 2018, 83, 13973-13980.

16. Rombola, M.; Rawal, V. H. Dicyclopentyl Dithiosquarate as an Intermediate for the Synthesis of Thiosquaramides. Org. Lett. 2018, 20, 514-517.

17. Kalbandkeri, R.G.; Mohamed, S.; Padma, D. K.; Murthy, A.R. A New Synthetic Procedure for the Preparation of Pyridinium Tetrafluoroborate and Hexafluorosilicate. Polyhedron, 1985, 4, 787-789.

18. Zhao, C.; Toste, D.; Raymond, K. N.; Bergman, R. G. Nucleophilic Substitution Catalyzed by a Supramolecular Cavity Proceeds with Retention of Absolute Stereochemistry, J. Am. Chem. Soc. 2014, 136, 14409-14412.

19. Allman, S.; Jensen, H.; Vijayakrishnan, B.; Garnett, J.; Leon, E.; Liu, Y.; Anthony, D.; Sibson, N.; Feizi, T.; Matthews, S.; Davis. B. A Potent Fluoro-Oligosaccharide Probes of Adhesion in Toxoplasmosis. ChemBioChem 2009, 10, 2522-2529.

20. Dempsey, K.; Mir, R.; Smajlagic, I.; Dudding, T. Expanding the Repertoire of Cyclopropenium Ion Phase Transfer Catalysis: Benzylic Fluorination. Tetrahedron 2018, 74, 3507-3511.

21. Li, L.; Huang, G.; Chen, Z.; Liu, W.; Wang, X.; Chen, Y.; Yang, L.; Li, W.; Li, Y. Gallium Trichloride Catalyzed Hydroamination of Alkynes: Scope, Limitation, and Mechanistic Studies by DFT. Eur. J. Org. Chem. 2012, 28, 5564-5572.

22. Fujita, H.; Kakuyama, S.; Kunishima, M. $N, N^{\prime}$-Dimethylated Benzyloxytriazinedione: A Stable Solid Reagent for Acid-Catalyzed $O$-Benzylation. Eur. J. Org. Chem. 2017, 4, 833839.

23. Malik, S.; Dixit, V.; Bharatam, P.; Kartha, R. A Simple, Mild, and Regioselective Method for the Benzylation of Carbohydrate Derivatives Promoted by Silver Carbonate. Carbohydr. Res. 2010, 345, 559-564.

24. APEX-II, Bruker AXS, Madison, Wisconsin, USA.

25. SAINT, Bruker AXS, Madison, Wisconsin, USA.

26. SADABS, Bruker AXS, Madison, Wisconsin, USA.

27. Sheldrick, G. M. Acta Cryst. A71, 3-8 (2015).

28. Sheldrick, G. M. Acta Cryst. C71, 3-8 (2015).

29. Spek, A. L. Acta Cryst. C71, 9-19 (2015).

30. SHELXTL, Bruker AXS, Madison, Wisconsin, USA, 2015.

31. Gaussian 09, Revision E.01, Frisch, M. J.; Trucks, G. W.; Schlegel, H. B.; Scuseria, G. E.; Robb, M. A.; Cheeseman, J. R.; Scalmani, G.; Barone, V.; Petersson, G. A.; Nakatsuji, H.; Li, X.; Caricato, M.; Marenich, A. V.; Bloino, J.; Janesko, B. G.; Gomperts, R.; Mennucci, B.; Hratchian, H. P.; Ortiz, J. V.; Izmaylov, A. F.; Sonnenberg, J. L.; Williams-Young, D.; Ding, F.; Lipparini, F.; Egidi, F.; Goings, J.; Peng, B.; Petrone, A.; Henderson, T.; Ranasinghe, D.; Zakrzewski, V. G.; Gao, J.; Rega, N.; Zheng, G.; Liang, W.; Hada, M.; Ehara, M.; Toyota, K.; Fukuda, R.; Hasegawa, J.; Ishida, M.; Nakajima, T.; Honda, Y.; Kitao, O.; Nakai, H.; Vreven, T.; Throssell, K.; Montgomery, J. A., Jr.; Peralta, J. E.; Ogliaro, F.; Bearpark, M. J.; Heyd, J. J.; Brothers, E. N.; Kudin, K. N.; Staroverov, V. N.; Keith, T. A.; Kobayashi, R.; Normand, J.; Raghavachari, K.; Rendell, A. P.; Burant, J. C.; Iyengar, S. S.; Tomasi, J.; Cossi, M.; Millam, J. M.; Klene, M.; Adamo, C.; Cammi, R.; 
Ochterski, J. W.; Martin, R. L.; Morokuma, K.; Farkas, O.; Foresman, J. B.; Fox, D. J. Gaussian, Inc., Wallingford CT, 2009.

32. Chai, J. D.; Head-Gordon, M. Long-Range Corrected Hybrid Density Functionals with Damped Atom-Atom Dispersion Corrections. Phys. Chem. Chem. Phys. 2008, 10, 6615-6620.

33. González, C.; Schlegel, H. B. Reaction Path following in Mass-Weighted Internal Coordinates. J. Phys. Chem. 1990, 94, 5523-5527.

34. Fukui, K. The Path of Chemical Reactions - the IRC Approach. Acc. Chem. Res. 1981, 14, 363-368.

35. Cancès, E.; Mennucci, B.; Tomasi, J. A New Integral Equation Formalism for the Polarizable Continuum Model: Theoretical Background and Applications to Isotropic and Anisotropic Dielectrics. J. Chem. Phys. 1997, 107, 3032-3041.

36. Yang. C.; Xue, X.-S.; Jin, J.-L.; Li, X.; Cheng, J.-P. Theoretical Study on the Acidities of Chiral Phosphoric Acids in Dimethyl Sulfoxide: Hints for Organocatalysis. J. Org. Chem. 2013, 78, 7076-7085.

37. Yang, C.; Xue, X.-S.; Li, X.; Cheng, J.-P. Computational Study on the Acidic Constants of Chiral Brønsted Acids in Dimethyl Sulfoxide. J.Org. Chem. 2014, 79, 4340-4351.

38. Legault, C. Y. CYLview, version 1.0b; Universite de Sherbrooke: Quebec, Canada, 2009, http://www.cylview.org

39. Dennington, R.; Keith, T. A.; Millam, J. M. GaussView, Version 6.1; Semichem Inc., Shawnee Mission, KS, 2016.

40. Zhurko, G. A; Zhurko, D. A. Chemcraft, version 1.8, http://www.chemcraftprog.com.

41. Grimme, S.; Goerigk, L.; Fink, R. F. Comput. Mol. Sci. 2011, 1, 211-228.

42. Grimme, S.; Antony, J.; Ehrlich, S.; Krieg, H. A. A Consistent and Accurate ab initio Parametrization of Density Functional Dispersion Correction (DFT-D) for the 94 Elements H-Pu. J. Chem. Phys. 2010, 132, 154104-154123.

43. Grimme, S.; Ehrlich, S.; Goerigk, L. Effect of the Damping Function in Dispersion Corrected Density Functional Theory. J. Comp. Chem. 2011, 32, 1456-1465.

44. Schrödinger Release 2021-1: Jaguar, Schrödinger, LLC, New York, NY, 2021.

45. Storer, J. W.; Giesen, D. J.; Cramer, C. J.; Truhlar, D. G. Class IV Charge Models: A New Semiempirical Approach in Quantum Chemistry. J. Comput.-Aided Mol. Des. 1995, 9, 87110.

46. Schrödinger Release 2021-1: MacroModel, Schrödinger, LLC, New York, NY, 2021. 